DOE/ER--1070-D1

DE92 010909

Environmental Restoration Division ORNL Environmental Restoration Program

\title{
Field Sampling and Analysis Plan for the Remedial Investigation of Waste Area Grouping 2 at Oak Ridge National Laboratory, Oak Ridge, Tennessee
}

\author{
H. L. Boston \\ T. L. Ashwood \\ D. M. Borders \\ V. Chidambariah \\ D. J. Downing \\ T. A. Fontaine \\ R. H. Ketelle \\ S. Y. Lee \\ D. E. Miller \\ G. K. Moore \\ G. W. Suter \\ M. F. Tardiff \\ J. A. Watts \\ D. S. Wicklifr
}

Manuscript Completed-November 1991

Date Issued-February 1992

Prepared by

Environmental Sciences Division

Oak Ridge National Laboratory

ESD Publication 3780

Prepared for

U.S. Department of Energy

Office of Environmental Restoration and Waste Management

under budget and reporting code EW 20

OAK RIDGE NATIONAL LABORATORY

Oak Ridge, Tennessee 37831-6285

managed by

MARTIN MARIETTA ENERGY SYSTEMS, INC.

for the

U.S. DEPARTMENT OF ENERGY

under contract DE-AC05-84OR21400 


\section{Author Affiliations}

H. L. Boston, T. L. Ashwood, T. A. Fontaine, S. Y. Lee, G. W. Suter, J. A. Watts, and D. S. Wickliff are members of the Environmental Sciences Division. D. Downing is a member of the Engineering Physics and Mathematical Division. R. H. Ketelle is a member of Energy Division. M. F. Tardiff is a member of the Environmental and Health Protection Division. All of the aforementioned divisions are part of Oak Ridge National Laboratory, Martin Marietta Energy Systems, Inc. D. M. Borders, G. K. Moore, and V. Chidambariah are Research Associates of The University of Tennessee, Knoxville. D. E. Miller is an employee of Automated Sciences Group, Oak Ridge. 


\section{CONTENTS}

TABLES $\ldots \ldots \ldots \ldots \ldots \ldots \ldots \ldots \ldots \ldots \ldots \ldots \ldots \ldots \ldots \ldots \ldots \ldots \ldots$

FIGURES $\ldots \ldots \ldots \ldots \ldots \ldots \ldots \ldots \ldots \ldots \ldots \ldots \ldots \ldots \ldots \ldots \ldots \ldots \ldots$

ACRONYMS $\ldots \ldots \ldots \ldots \ldots \ldots \ldots \ldots \ldots \ldots \ldots \ldots \ldots \ldots \ldots \ldots \ldots \ldots \ldots \ldots$

EXECUTTVE SUMMARY $\ldots \ldots \ldots \ldots \ldots \ldots \ldots \ldots \ldots \ldots \ldots \ldots \ldots \ldots$

1. INTRODUCTION $\ldots \ldots \ldots \ldots \ldots \ldots \ldots \ldots \ldots \ldots \ldots \ldots \ldots \ldots \ldots \ldots$

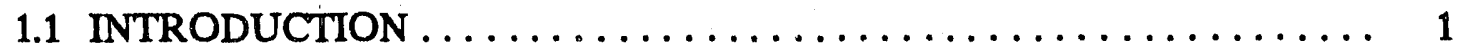

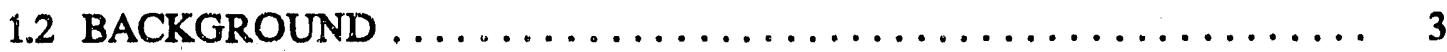

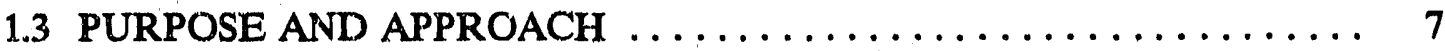

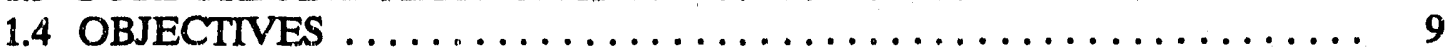

2. SCOPE AND IMPLEMENTATION $\ldots \ldots \ldots \ldots \ldots \ldots \ldots \ldots \ldots \ldots$

2.1 CONTAMINANTS AND PATHWAYS OF CONCERN $\ldots \ldots \ldots \ldots \ldots 11$

2.1.1 Human Health Risk ...................... 11

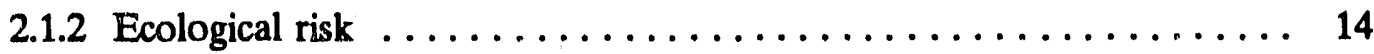

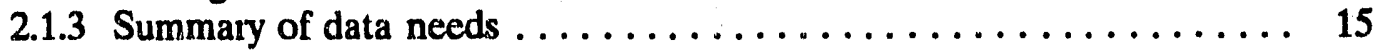

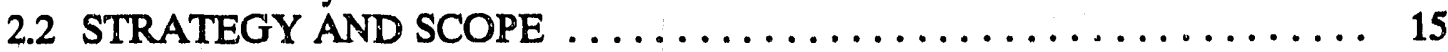

2.3 S\&A ORGANIZATION AND IMPLEMENTATION ........... 20

2.3.1 External Data Sources ...................... 20

2.3.2 Organization of the Staff for S\&A Activities ............ 25

2.4 STATISTICAL SUPPORT ........................ 29

2.5 QUALITY ASSURANCE/QUALITY CONTROL PLAN . . . . . . . 30

2.5.1 Project Requirements for Q.A Awareness and Training $\ldots \ldots \ldots \ldots 34$

2.5.2 QA for Data from Other Projects .................. 34

2.5.3 QA Objectives for Measurement of Data .............. 34

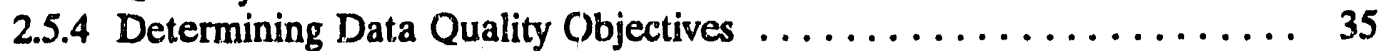

2.5.5 Data Quality Parameters ....................... 36

2.5 .6 Field Activities $\ldots \ldots \ldots \ldots \ldots \ldots \ldots \ldots \ldots \ldots \ldots \ldots \ldots \ldots$

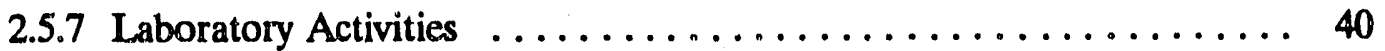

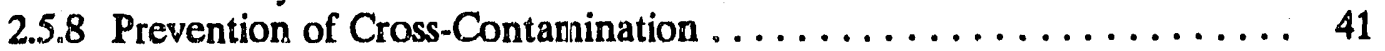

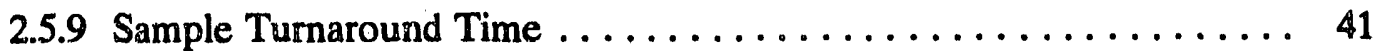

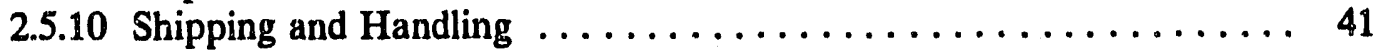

2.5 .11 Variance System ...................... 41

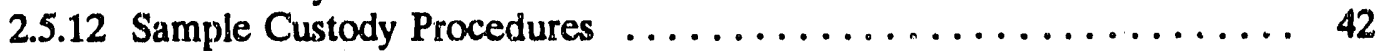

2.5 .13 Analytical Procedures ...................... 42

2.5.14 Data Summarization, Validation, and Reporting ........... 44

2.5.15 Internal QC Checks and Frequency ................ 46

2.5.16 QA Reports to Managment .................. 46

3. SEDIMENT SAMPLING PLAN $\ldots \ldots \ldots \ldots \ldots \ldots \ldots \ldots \ldots \ldots \ldots$ 
3.1 INTRODUCIION ............................. 49

3.2 OBJECTIVES AND APPROACH $\ldots \ldots \ldots \ldots \ldots \ldots \ldots \ldots \ldots .51$

3.2.1 Objective 1-Floodplain Radiological Walkover and Stream Sediment

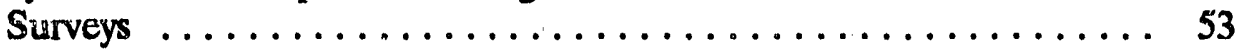

3.2.2 Sampling and Analysis for Objective $1 \ldots \ldots \ldots \ldots \ldots \ldots \ldots, 54$

3.2.3 Objective 2-Sampling of Contaminant Transport During Storms .... 55

3.2.4 Sampling and Analysis for Objective 2 $\ldots \ldots \ldots \ldots \ldots \ldots \ldots .57$

3.2.5 Objective 3--Quantifying and Tracking Contaminant Inventories in Stream Sediments and Identifying Contaminant Sources ........ 60

3.2.6 Sampling and Analysis for Objective $3 \ldots \ldots \ldots \ldots \ldots \ldots \ldots 63$

3.2.7 Objective 4-Develop Models to Predict Sediment Transport for the wOC Watershed ............................. 64

3.2.8 Sampling and Analysis for Objective $4 \ldots \ldots \ldots \ldots \ldots \ldots \ldots, 66$

3.2.9 Support from the ERMA Program $\ldots \ldots \ldots \ldots \ldots \ldots \ldots \ldots .67$

3.3 SAMPLING AND ANALYTICAL METHODS $\ldots \ldots \ldots \ldots \ldots \ldots \ldots, 68$

3.4 FUTURE EFFORTS $\ldots \ldots \ldots \ldots \ldots \ldots \ldots \ldots \ldots \ldots \ldots \ldots \ldots, 68$

4. SURFACE WATER SAMPLING PROGRAM $\ldots \ldots \ldots \ldots \ldots \ldots \ldots \ldots, 73$

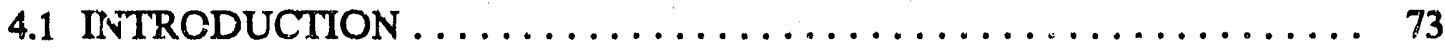

4.1.1 Main Channels ........................... 73

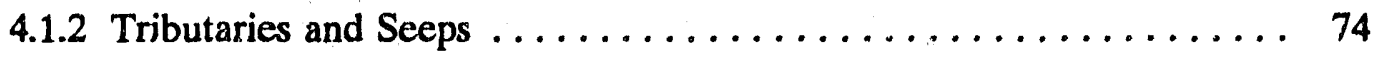

4.1.3 Review of Existing Data $\ldots \ldots \ldots \ldots \ldots \ldots \ldots \ldots \ldots \ldots \ldots \ldots \ldots \ldots$

4.2 OBJECTIVES AND APPKOACH $\ldots \ldots \ldots \ldots \ldots \ldots \ldots \ldots \ldots, 77$

4.2.1 Surface Water Sampling (Main Channels) $\ldots \ldots \ldots \ldots \ldots \ldots \ldots \ldots \ldots \ldots$

4.2.2 Sampling and Analysis for Surface Water ............... 78

4.2.3 Tributary Sampling Program $\ldots \ldots \ldots \ldots \ldots \ldots \ldots \ldots \ldots \ldots \ldots \ldots \ldots$

4.2.4 Sampling and Analysis for Tributaries $\ldots \ldots \ldots \ldots \ldots \ldots \ldots \ldots, 80$

4.2.5 Seep Sampling Program $\ldots \ldots \ldots \ldots \ldots \ldots \ldots \ldots \ldots \ldots \ldots \ldots \ldots \ldots \ldots$

4.2.6 Sampling and Analysis for Seeps $\ldots \ldots \ldots \ldots \ldots \ldots \ldots \ldots \ldots, 83$

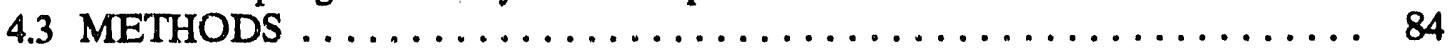

4.3.1 Surface Water $\ldots \ldots \ldots \ldots \ldots \ldots \ldots \ldots \ldots \ldots \ldots \ldots, 84$

4.3.2 Tributaries and Seeps $\ldots \ldots \ldots \ldots \ldots \ldots \ldots \ldots \ldots \ldots \ldots, 86$

4.4 QUALITY ASSURANCE $\ldots \ldots \ldots \ldots \ldots \ldots \ldots \ldots \ldots \ldots \ldots, 86$

5. GROUNDWATER SAMPLING PLAN $\ldots \ldots \ldots \ldots \ldots \ldots \ldots \ldots \ldots, 89$

5.1 INTRODUCTION $\ldots \ldots \ldots \ldots \ldots \ldots \ldots \ldots \ldots \ldots \ldots \ldots \ldots \ldots, \quad 89$

5.1.1 Review of Existing Data $\ldots \ldots \ldots \ldots \ldots \ldots \ldots \ldots \ldots \ldots, 89$

5.1 .2 Objectives ............................. 89

5.1 .3 Approach $\ldots \ldots \ldots \ldots \ldots \ldots \ldots \ldots \ldots \ldots \ldots \ldots \ldots \ldots \ldots \ldots$

5.2 WATER COLLECTION, FIELD MEASUREMENTS, AND

SOURCE IDENTIFICATION ....................... 90

5.2.1 Well Sampling and Water Quality Analyses .............. 90

5.2.2 WAG 2 Geology, Well Construction, and Water Level Data Interpretation $\ldots \ldots \ldots \ldots \ldots \ldots \ldots \ldots \ldots \ldots \ldots \ldots \ldots, 93$

5.2.3 Evaluation of Data for Groundwater Geochemistry ........... 93

5.2 .4 Hydrogeologic Investigations $\ldots \ldots \ldots \ldots \ldots \ldots \ldots \ldots \ldots \ldots, 94$

5.2 .5 Stormflow Zone $\ldots \ldots \ldots \ldots \ldots \ldots \ldots \ldots \ldots \ldots \ldots \ldots \ldots$ 
6. ECOLOGICAL ASSESSMENT $\ldots \ldots \ldots \ldots \ldots \ldots \ldots \ldots \ldots \ldots \ldots, 97$

6.1 INTRODUCTION $\ldots \ldots \ldots \ldots \ldots \ldots \ldots \ldots \ldots \ldots \ldots \ldots \ldots \ldots \ldots \ldots \ldots$

6.1.1 Overview $\ldots \ldots \ldots \ldots \ldots \ldots \ldots \ldots \ldots \ldots \ldots \ldots \ldots, 97$

6.1.2 Assessment Endpoints ........................ 97

6.2 REVIEW OF EXISTING DATA $\ldots \ldots \ldots \ldots \ldots \ldots \ldots \ldots \ldots \ldots \ldots \ldots \ldots$

6.2.1 Conceptual Model $\ldots \ldots \ldots \ldots \ldots \ldots \ldots \ldots \ldots \ldots \ldots \ldots, 9, \ldots$

6.2.2 Radiological Contaminants ...................... 100

6.2.3 Nonradiological Contaminants $\ldots \ldots \ldots \ldots \ldots \ldots \ldots \ldots \ldots, 102$

6.2.4 Data Needs ............................. 102

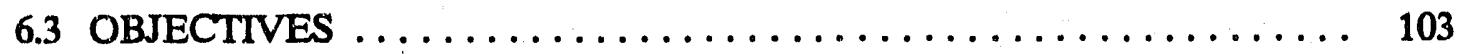

6.4 BIOLOGICAL SAMPLING $\ldots \ldots \ldots \ldots \ldots \ldots \ldots \ldots \ldots \ldots \ldots \ldots \ldots \ldots \ldots \ldots$

6.4.1 Task 1: Scoping Survey of Contaminant Levels in Selected Wildlife . 104

6.4.2 Task 2: Threatened and Endangered Species Surveys .......... 105

6.4.3 Task 3: Mink and Kingfisher Sampling $\ldots \ldots \ldots \ldots \ldots \ldots \ldots \ldots, 105$

6.4.4 Task 4: Contaminant Fate Model .................... 106

6.4.5 Task 5: Toxicity Testing and Risk Review ............. 106

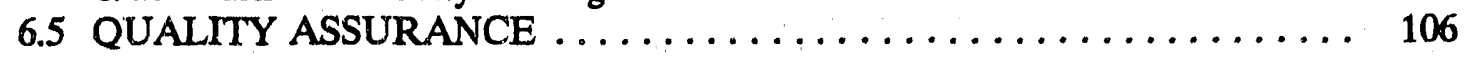

7. SAMPLE TRACKING AND RECORDS MANAGEMENT $\ldots \ldots \ldots \ldots \ldots 109$

7.1 INTRODUCTION $\ldots \ldots \ldots \ldots \ldots \ldots \ldots \ldots \ldots \ldots \ldots \ldots \ldots \ldots \ldots, 109$

7.2 OBJECTIVES .............................. 109

7.3 FIELD DATA MEASUREMENTS AND OBSERVATIONS $\ldots \ldots \ldots \ldots 110$

7.3.1 Loghooks . ............................. 110

7.3.2 Sample Custody ......................... 110

7.4 ANALYTICAL LABORATORY DATA ................ 111

7.5 SIMULATION AND RISK ASSESSMENT MODELS AND DATA $\ldots . \quad 111$

7.6 DATA MANAGEMENT $\ldots \ldots \ldots \ldots \ldots \ldots \ldots \ldots \ldots \ldots \ldots \ldots \ldots \ldots$

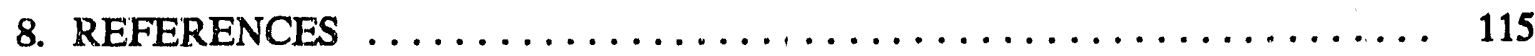

ACKNOWLEDGMENTS $\ldots \ldots \ldots \ldots \ldots \ldots \ldots \ldots \ldots \ldots \ldots \ldots \ldots \ldots$

APPENDIX A: WAG 2 RI STAFF $\ldots \ldots \ldots \ldots \ldots \ldots \ldots \ldots \ldots \ldots \ldots \ldots \ldots \ldots \ldots \ldots$

APPENDIX B: TYPE OF DATA REQUIRED FOR HSPF
MODEL OF SEDIMENT TRANSPORT $\ldots \ldots \ldots \ldots \ldots \ldots$ B-1

APPENDIX C: WAG 2 PROCEDURES $\ldots \ldots \ldots \ldots \ldots \ldots \ldots \ldots \ldots$, C-1 


\section{TABLES}

Table No.

2.1 Monitoring and investigations that support the

WAG 2 RI Project $\ldots \ldots \ldots \ldots \ldots \ldots \ldots \ldots \ldots \ldots \ldots$

2.2 Data product hierarchy for the WAG 2 RI Plan

field sampling and analysis program $\ldots \ldots \ldots \ldots \ldots \ldots \ldots \ldots$

3.1 Estimated inventories of ${ }^{137} \mathrm{Cs},{ }^{60} 60$, and

${ }^{90} \mathrm{~S} r$ in the abiotic and biotic compartments

of White Oak Lake . . . . . . . . . . . . . . . . . . . 50

3.2 Methods for collection and number of samples

for aquatic sediments and floodplain soils $\ldots \ldots \ldots \ldots \ldots$

Analytical Methods for sediment samples $\ldots \ldots \ldots \ldots \ldots \ldots \ldots$

4.1 Surface water sampling and analysis at White Oak Dam $\ldots \ldots \ldots 79$

4.2 Initial scoping sampling to determine contaminant flux into

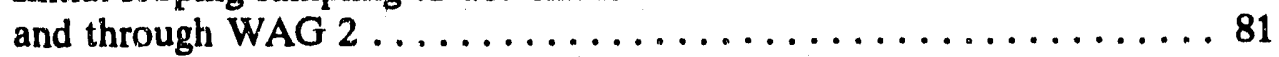

Analysis methods for surface water $\ldots \ldots \ldots \ldots \ldots \ldots$

4.4 Methods for initial scoping study of tributaries and seeps $\ldots \ldots \ldots \ldots \ldots \ldots \ldots \ldots \ldots \ldots \ldots$

5.1 ORNL WAG perimeter well analyses $\ldots \ldots \ldots \ldots \ldots \ldots \ldots \ldots$

6.1 Samples and analyses to support biological sampling in WAG $2 \ldots \ldots 7$

7.1 Data to be included in the WAG 2 sampling and analysis numeric data base and the originating office $\ldots \ldots \ldots \ldots \ldots \ldots \ldots \ldots$ 


\section{FIGURES}

Fig. No.

2.3 Monitoring stations and perimeter wells in the White Oak

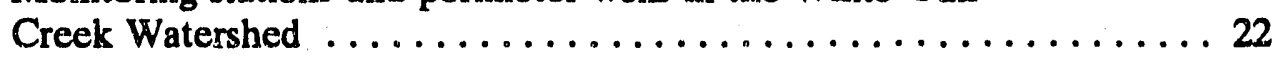

Project organization chart for WAG 2 RI $\ldots \ldots \ldots \ldots \ldots$

Sampling points along a hypothetical hydrograph $\ldots \ldots \ldots \ldots \ldots$

$3.4 \quad$ Stream gravel ${ }^{137} \mathrm{Cs}$ and ${ }^{90} \mathrm{Sr}$ survey data sampled in $1985 \ldots \ldots \ldots 6$

4.1 Main channel and tributary surface water monitoring stations ..... 75

Locations of WAG 2 perimeter wells $\ldots \ldots \ldots \ldots \ldots \ldots \ldots \ldots$

6.1 Biological monitoring and sampling programs at ORNL $\ldots \ldots \ldots 98$

6.2 Conceptual model of contaminant movement through the WAG 2

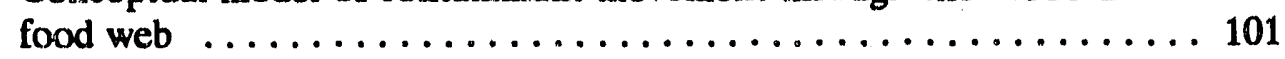

7.1 Steps in processing data to be included in the WAG 2 numeric data base 


\section{ACRONYMS}

\begin{tabular}{|c|c|}
\hline APO & Analytical Program Office \\
\hline ASCE & American Society of Civil Engineers \\
\hline ARAR & applicable or relevant and appropriate requirement \\
\hline ASC & Analytical Services Coordinator \\
\hline ASEMP & Active Sites Environmental Monitoring Program \\
\hline BMAP & Biological Monitoring and Abatement Program \\
\hline CERCLA & Comprehensive Environmental Response, Compensation, and Liability Act \\
\hline CLP & Contract Laboratory Program \\
\hline CRRI & Clinch River RI \\
\hline DMC & Document Management Center \\
\hline DOE & Department of Energy \\
\hline DQO & Data Quality Objective \\
\hline EPA & Environmental Protection Agency \\
\hline ER & environmental restoration \\
\hline ERD & Environmental Restoration Division \\
\hline ERMA & environmental restoration monitoring and assessment \\
\hline ESD & Environmental Sciences Division \\
\hline ESP & Environmental Surveillance Program \\
\hline FFA & Federal Facility Agreement \\
\hline FS & Feasibility Study \\
\hline HHMS & Hydraulic Head Measuring Stations \\
\hline HRE & Homogeneous Reactor Experiment \\
\hline HSPF & Hydrologic Simulation Program-Fortran \\
\hline ICP & inductively coupled plasma \\
\hline IG & intrinsic germanium \\
\hline LCS & laboratory control sample \\
\hline NBS & National Bureau of Standards \\
\hline $\mathrm{NDB}$ & Numeric Data Base \\
\hline NPDES & National Pollutant Discharge Elimination System \\
\hline OHER & Office of Environmental and Health Research \\
\hline ORAU & Oak Ridge Associated Universities \\
\hline ORNL & Oak Ridge National Laboratory \\
\hline ORRHAGS & Oak Ridge Reservation Hydrology and Geology Studies \\
\hline PCB & polychlorinated biphenyl \\
\hline PE & performance evaluation \\
\hline QAS & quality assurance specialist \\
\hline RCRA & Resource Conservation and Recovery Act \\
\hline $\mathbf{R f D}$ & reference dose factor \\
\hline RFI & RCRA facility investigation \\
\hline RI & remedial investigation \\
\hline RI/FS & remedial investigation/feasibility study \\
\hline SAS & Statistical Analysis System \\
\hline S\&A & sampling and anaiysis \\
\hline SIMS & Sample Information Management System \\
\hline SOP & standard operating procedure \\
\hline TDEC & Tennessee Department of Environment and Conservation \\
\hline
\end{tabular}


TVA Tennessee Valley Authority

TWRA Tennessee Wildlife Resources Agency

USGS U.S. Geological Survey

USRADS Ultrasonic Ranging and Data System

VOC volatile organic compound

WAG waste area grouping

WOC White Oak Creek

WOCE White Oak Creek Embayment

WOL White Oak Lake 


\section{EXECUTIVE SUMMARY}

This field sampling and analysis (S\&A) plan has been developed as part of the Department of Energy's (DOE's) remedial investigation (RI) of Waste Area Grouping (WAG) 2 at Oak Ridge National Laboratory (ORNL) located in Oak Ridge, Tennessee. The S\&A plan has been written in support of the remedial investigation (RI) plan for WAG 2 (ORNL 1990).

WAG 2 consists of White Oak Creek (WOC) and its tributaries downstream of the ORNL main plant area, White Oak Lake (WOL), White Oak Creek embayment (WOCE) on the Clinch River, and the associated floodplain and subsurface environment. The WOC system is the surface drainage for the major ORNL WAGs and has been exposed to a diversity of contaminants from operations and waste disposal activities in the WOC watershed. WAG 2 acts as a conduit through which hydrologic fluxes carry contaminants from upgradient areas to the Clinch River. Water, sediment, soil, and biota in WAG 2 are contaminated and continue to receive contaminants from upgradient WAGs.

The remediation of WAG 2 logically follows the cessation of contaminant input from hydrologically upgradient WAGs. Remedial Investigations and remediations are underway or planned for contaminated areas upgradient of WAG 2. In the interim, the long term S\&A strategy takes full advantage of WAG 2's role as an integrator of contaminant fluxes from other ORNL WAGs and focuses on four key goals:

1. Implement, in concert with other programs, longuterm monitoring and tracking of contaminants leaving other WAGs, entering WAG 2 , and being transported off-site.

2. Provide a conceptual framework to integrate and develop information at the watershedlevel for pathways and processes that are key to contaminant movement, and so support remedial efforts at ORNL.

3. Provide periodic updates of estimates of potential risk (both human health and ecological) associated with contaminants accumulating in and moving through WAG 2 to off.site areas.

4. Through long-term monitoring a.nd continually updated iisk assessments, support the ER prioritization and evaluation of remedial actions.

The general objectives of the S\&A plan are to support a multimedia environmental monitoring and characterization program to (1) define and monitor the input of contaminants from adjacent WAGs; (2) support a mass-balance approach to determining sources, sinks, and tiansport of contaminants in WAG 2 based on hydrologic fluxes; (3) document long-term trends in contaminant pools and fluxes; and (4) develop models that predict potential contaminant releases under future conditions.

The goals of the preliminary stages of the S\&A Plan (i.e., the first 2 years of efforts described herein) are: (1) scoping and screening studies to form a basis for statistical design of longer-term sampling and monitoring programs; (2) development of proceduras, selection of sites, and initial implementation of the monitoring and tracking efforts; and (3) collecting information for components and contaminants for which few data exist to update the 
preliminary evaluations of human health and ecological risk. Data from surveys and initial sampling efforts will be used to stratify the system for later sampling efforts, to estimate the initial level of variance for parameters of interest, and to determine the cost required to reduce the uncertainty in the estimates of risk. These activities support the four key goals listed above as well as supporting the preliminary components of the RI/FS for WAG 2 (i.e., preliminary site characterization, updated estimates of health and ecological risk, developing a site conceptual model, and identifying operable units).

A risk-based contaminant screening for WAG 2 and information for adjacent areas indicate that a number of contaminants pose significant concern for risk to human health and the environment. WAG 2 is currently under institutional control, and access is restricted. However, contaminants are currently being released from WAG 2 to off-site areas, and in the event that institutional control would be lost, contaminants in WAG 2 would constitute a highly significant risk to future occupants. The S\&A plan will focus initially on key radionuclides $\left({ }^{137} \mathrm{Cs},{ }^{60} \mathrm{Co},{ }^{3} \mathrm{H}\right.$, and $\left.{ }^{50} \mathrm{Sr}\right)$. These radionuclides are among the major contributors to human health risk, some can easily be surveyed in the field (e.g., ${ }^{137} \mathrm{Cs}$ and ${ }^{60} \mathrm{Co}$ ), and all four can easily be measured in the laboratory. Fewer samples will be analyzed for metals, organies, and other radiological contaminants (e.g., transuranics, ${ }^{99} \mathrm{Tc},{ }^{152,154} \mathrm{Eu}$ ). We will determine if data for selected radionuclides can be used to guide future sampling for other contaminants of concern.

Contaminants of Concern. Floodplain soils and aquatic sediments contain large quantities of contaminants. Most of the major contaminants in WAG 2 are particle reactive and are found associated with soils and sediments. Data for soils and sediments are available for few areas in WAG 2 and are more extensive for gamma emitting radionuclides than for metals, organic contaminants, or other radiological contaminants. External exposure from radionuclides $\left({ }^{137} \mathrm{Cs}\right.$ and ${ }^{60} \mathrm{Co}$ ) in sediments is a high priority for further evaluation (i.e., potential excess lifetime cancer risk $>10^{-4}$ ). The primary risk to off-site areas results from the transport of ${ }^{137} \mathrm{Cs}$ in sediments.

Surface water in WAG 2 meets ORNL's National Pollutant Discharge Elimination System (NPDES) permit requirements; however, a risk-based contaminant screening indicated that ${ }^{90} \mathrm{Sr},{ }^{3} \mathrm{H}$, and polychlorinated biphenyls (PCBs) are concerns for human health. Additional data are also needed for ${ }^{152} \mathrm{Eu},{ }^{154} \mathrm{Eu},{ }^{137} \mathrm{Cs}$, ${ }^{235} \mathrm{U}$, arsenic, and thallium. Because groundwater discharges to surface water prior to leaving the WOC watershed, surface water is an important point of exposure to groundwater contaminants.

Groundwater in WAG 2 has localized contamination by radionuclides, organics, and metals. Tritium, ${ }^{90} \mathrm{Sr}$, and lead in groundwater are of concern for human health. Additionally, data are required for all classes of contaminants in groundwater, as well as data for groundwater pathways and fluxes.

Biota in WAG 2 have accumulated organic, inorganic, and radiological contaminants. Some chemicals in surface water and sediments occurred at concentrations that are potentially toxic to sensitive species. Other chemicals that were not detected had detection limits that were higher than toxic thresholds. However, data from species surveys, bioaccumulation monitoring, and ambient toxicity testing suggest that severe effects are not occurring in the aquatic habitats of WAG 2. Additional data for nonradiological contaminants for aquatic biota and data for all classes of contaminants in terrestrial biota are needed. 
PCBs in fish were high priority for human health risk. Additional information is also required for ${ }^{137} \mathrm{Cs}$ and mercury in fish.

In analyses for organic (semivolatile and nonvolatile) compounds, many compounds were not detected (i.e., they were not present or were present at a concentration below the analytical limit of detection). A screening was conducted for those compounds by assuming that they were present at a concentration equal to the analytical detection limit. For some compounds, the screening exercise found that the compound would have be classed as high priority for additional investigation had it been present at the lower limit of detection (Blaylock et al. 1991b). At this time we have no evidence that any of those compounds was present; therefore, either analytical methods with lower limits of detection will be used, or individual compounds will be eliminated from further consideration based on evidence such as no reported detection in any media and no known sources in the watershed.

Approach WAG 2 is complex and dynamic with diverse sources of contaminants and fluxes driven by changing environmental conditions. Rather than an exhaustive site characterization for all contaminants, media, and flow pathways, we will (1) focus on contaminants and pathways of greatest concern and (2) monitor and gather sufficient information for processes controlling or driving contaminant fluxes to construct an appropriate conceptual model for contaminant pools and fluxes in WAG 2 (see Chapter 2). This approach is analogous to the Observational Approach and allows the early identification of remedial alternatives and focuses efforts on the gathering of data useful for evaluating alternatives to reduce risk.

The general objectives and goals of the preliminary stages of the S\&A plan have been listed previously. As noted, the initial efforts will focus on selected radionuclides that are major contributors to human health risks, and fewer samples will be analyzed for metals, organics and other radionuclides. This approach allows us to focus on primary sources of risk, and will allow us to determine if data for selected radionuclides can be used to guide future sampling for other contaminants of concern.

Much of the effort during the first 2 years will focus on sediments, seeps, and tributaries. Sediments are the primary pool for all contaminants of concern, except ${ }^{3} \mathbf{H}$, in WAG 2, and contaminants are transported off-site with sediments during high discharge events. Sediment and ccntaminant transport during storms will be quantified, and preliminary inventories of major contaminants in sediments will be developed. Models will be developed in collaboration with other projects to predict contaminant input into WAG 2, movement within WAG 2, and transport to the Clinch River. Hydrologically driven contaminant transport models are needed because contaminant transport will vary due to natural environmental factors (e.g., precipitation), changing land use in the watershed (e.g., increased paving), and remedial activities (e.g., capping) in upgradient WAGs.

Seeps and tributaries provide useful points at which to detect, quantify, and monitor contarninant input from adjacent WAGs to WAG 2. Seeps are especially important as connections between contaminated groundwater and surface water in WAG 2. Seeps and tributaries contributing to contaminant fluxes will be identified and monitored.

Data now becoming available from environmental monitoring efforts at ORNL will be used to evaluate the nature and extent of groundwater contamination and to determine the 
pathways of groundwater transport. Hydrogeologic investigations will evaluate the importance of groundwater flow from adjacent WAGs through WAG 2 to discharge in local streams. Data provided by the seep sampling program will also support the groundwater efforts.

We will gather preliminary data for contaminants in biota (e.g., PCBs) that (1) are important for potential human health risk, (2) are needed to update estimates of ecological risk, and (3) can be useful as indicators of contaminant availability in the system. WAG 2 efforts will serve as a focal point for ecological assessment in the White Oak Creek watershed.

Monitoring activities at ORNL [e.g., Biological Monitoring and Abatement Program, NPDES, and environmental surveillance surface water and groundwater monitoring] provide an important source of information as well as opportunities for collaboration on data collection. Activities related to environmental restoration [e.g., RIs and Resource Conservation and Recovery Act (RCRA) facility investigations (RFIs)] underway in the WOC watershed and the Clinch River (Off-Site ER Program) by necessity are linked to the WAG 2 project. Data from all activities are being evaluated for utility and acceptability under WAG 2 RI data quality objectives. These projects and monitoring programs are being integrated with the WAG 2 RI S\&A efforts.

The monitoring program will support a mass-balance (i.e., input, storage, and release) approach for discrete reaches of WAG 2 . Reaches will be subdivided as required based on contaminant input, inventory, and potential remedial action. This approach transiates to the identification of operable units to be considered for corrective measures or eliminated from further efforts. Data from these efforts will be used to update the risk assessment and refine the remedial planning approach. The multimedia environmental monitoring effort will evolve as new information becomes available and as needs and conditions change. 


\title{
1. INTRODUCTION
}

\author{
H. L. Boston and T. L. Ashwood
}

\subsection{INTRODUCTION}

This field sampling and analysis (S\&A) plan has been developed as part of the Department of Energy's (DOE's) remedial investigation (RI) of Waste Area Grouping (WAG) 2 at Oak Ridge National Laboratory (ORNL) located in Oak Ridge, Tennessee. The S\&A plan has been written in support of the remedial investigation (RI) plan for WAG 2 (ORNL 1990).

WAG 2 consists of White Oak Creek (WOC) and its tributaries downstream of the ORNL main plant area, White Oak Lake (WOL), White Oak Creek embayment (WOCE) on the Clinch River, and the associated floodplain and subsurface environment (Fig. 1.1). The WOC system is the surface drainage for the major ORNL WAGs and has been exposed to a diversity of contaminants from operations and waste disposal activities in the WOC watershed. WAG 2 acts as a conduit through which hydrologic fluxes carry contaminants from upgradient areas to the Clinch River. Water, sediment, soil, and biota in WAG 2 are contaminated and continue to receive contaminants from upgradient WAGs.

Remedial investigations and remediations are under way or planned for contaminated areas hydrologically upgradient of WAG 2; therefore, contaminant inputs will change as individual upgradient areas are remediated. Because remediations undertaken in WAG 2 in the short term could be negated by future contaminant input, implementation of corrective measures in WAG 2 should follow the completion of remediation of upgradient WAGs. However, because the WOC system acts as a conduit for contaminants from upgradient areas and because WAG 2 has accumulated contaminants that may represent near-term hazards, a phased remedial investigation in WAG 2 is beginning immediately.

The RI plan for WAG 2 (ORNL 1990), submitted in December 1990, presents a strategy that takes advantage of the location of WAG 2 as a conduit and integrator of contaminant fluxes irom the other ORNL WAGs. To take full advantage of WAG 2's role as an integrator of contaminants from other ORNL WAGs, we have developed a long-term S\&A strategy aimed at four key goals.

1. Implement, in concert with rther programs, long-term monitoring of contaminants leaving other WAGs, entering WAG 2, and being transported off-site.

2. Provide a conceptual framework for addressing watershed-level processes and effects.

3. Provide periodic updates of the potential risk (both ecological and human health) associated with contaminants flowing through WAG 2 to off-site areas.

4. Through long-term monitoring and continually updated risk assessments, support the ER prioritization of remedial actions. 


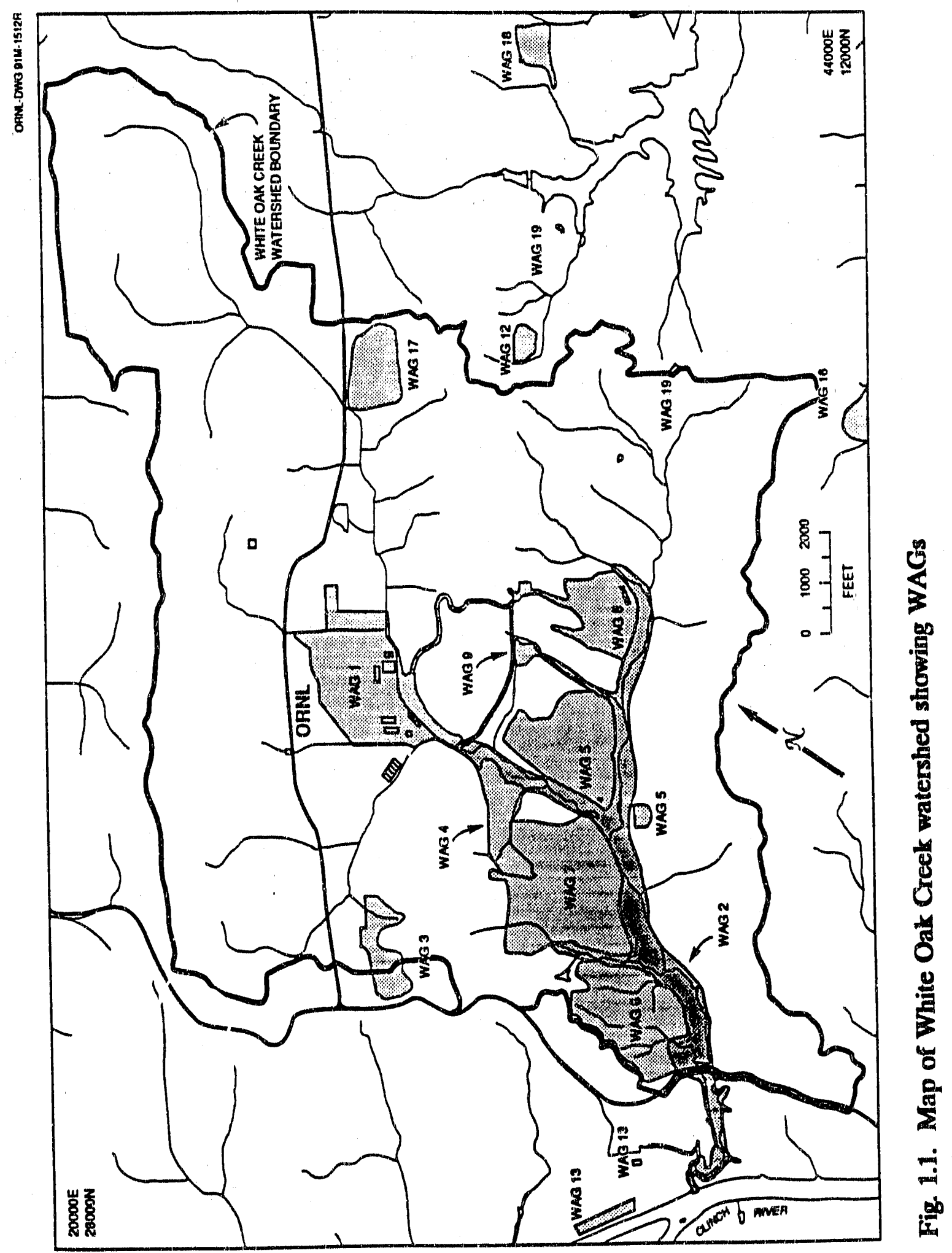


This document is organized in the following manner:

- Chapter 1 presents an overview of the RI plan, background information for the WAG 2 system, and objectives of the S\&A plan.

- Chapter 2 presents the scope and implementation of the first 2 years of effort of the S\&A plan and includes recent information about contaminants of concern, organization of S\&A activities, interactions with other programs, and quality assurance specific to the S\&A activities.

- Chapters 3-6 provide details of the field sampling plans for sediment, surface water, groundwater, and biota, respectively.

- Chapter 7 describes the sample tracking and records management plan.

The proposed schedule for the WAG 2 RI (Fig. 1.2) has been divided into three phases: Phase 1 insists of submission of the RI plan and a scoping survey of the site to determine the need for interim corrective measures, Phase II includes the multimedia environmental monitoring program and preliminary stages of site characterization to be conducted during the period in which remedial efforts were underway in upgradient WAGs (the interim 10 to 15 year period), and Phase III consists of the formal components of a typical RI consistent with CERCLA. Phase I has been partially completed with submission of the RI plan. As noted in the plan, the complexity of the WAG 2 system did not allow completion of the preliminary contaminant screening analysis or formulation of a detailed S\&A plan at the time the document was submitted. The WAG 2 RI work plan will not be a prototypic work plan but will be developed and submitted in stages during the period that upgradient WAGs are being remediated as new information becomes available and as needs and conditions change. The RI plan contained an outline of the initial componenis of the S\&A plan with the acknowledgement that updated versions of the S\&A plan for the multimedia environmental monitoring program and preliminary stoges of site characterization would be provided as addenda to the initial plan. This report provides the second iteration of the S\&A plan for the WAG 2 RI project and updates material in the RI plan (ORNL 1990).

This iteration of the S\&A plan provides details of activities to be conducted during FY 1992 and 1993. During this period we will develop the foundation of the multimedia environmental monitoring program and begin to gather information to support eventual site characterization. This plan will be updated every 12 to 24 months as new information is gathered and the contaminant screening is updated. We recognize that the U.S. Environmental Protection Agency (EPA) and the Tennessee Department of Environment and Conservation (TDEC) have reserved the right to require modifications at any stage of these efforts. Furthermore, we recognize that availability of funding due to changes in prioritization under the Federal Facility Agreement (FFA) may affect the schedule of activities.

\section{BACKGROUND}

The WAG 2 RI Plan (ORNL 1990) reviewed data for contaminants in WAG 2. This section summarizes the salient findings of that review and notes preliminary data needs. Past practices have resulted in the widespread contamination of WAG 2. Discharges and releases from existing operations and contaminated areas are a continuing source of contaminants to 


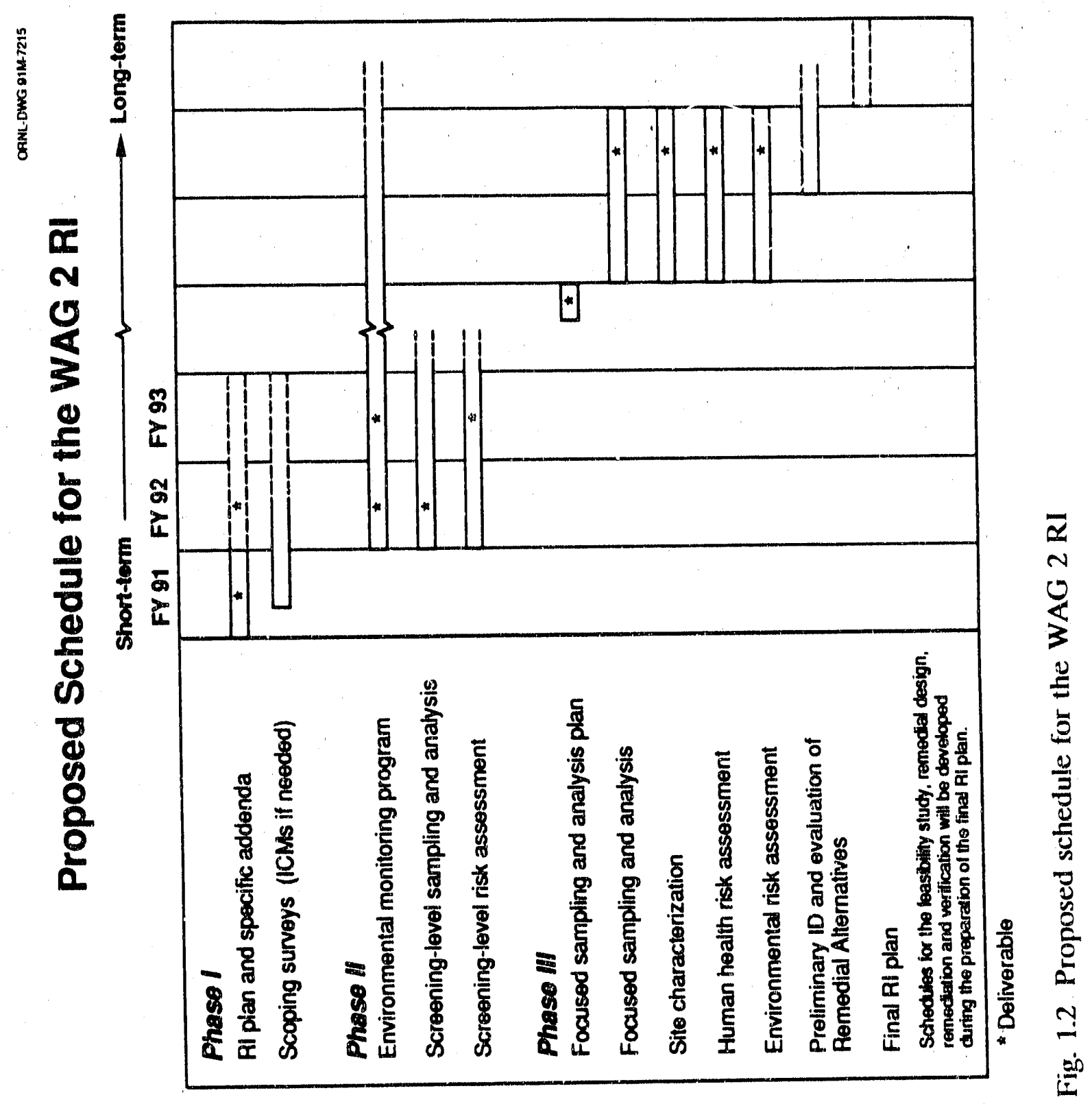


WAG 2 (see ORNL [1990] Sects. 5 and 6). Although substantial information exists for some radiological contaminants in some areas and media for other areas, and media data are frequently not available or insufficient. Data for nonradiological contaminants are generally sparse. Because WAG 2 is actively receiving contaminants and releasing contaminants to the Clinch River (off-site), we need information on contaminant pathways and fluxes. We need to develop the means to monitor those fluxes important for human health and environmental risk considerations. In addition, we need models to link contaminant transport through the watershed and to predict contaminant releases under future conditions and to evaluate risks to off-site areas.

Surface water quality. Surface water quality is monitored at several locations in the WOC watershed (see Fig. 2.1, Table 2.1, and ORNL [1990], see Sect. 4). Although surface water quality in WAG 2 generally meets the National Pollutant Discharge Elimination System (NPDES) permit requirements, the concentration of some metals are elevated above background, and high concentrations of ${ }^{3} \mathrm{H}$ and ${ }^{90} \mathrm{Sr}$ in streams in the Melton Branch arm of the drainage system would constitute a human health risk if consumed (Sect. 2.1). Thus, contaminant inputs need to be quantified (e.g., monitoring of seeps and tributaries), and the fate of key contaminants need to be determined. Additional data are needed for metals and organic contaminants from all reaches.

Soiks, sediments, and sediment transport. Although contaminants primarily enter WAG 2 in soluble form, most of the contaminants of concern in WAG 2 are particle reactive (e.g., ${ }^{137} \mathrm{Cs},{ }^{60} \mathrm{Co}$, polychlorinated byphenyls [PCBs], lead, mercury) and so are found associated with aquatic and floodplain sediments. Contaminated sediments can be mobilized during high discinarge events and by human activities. Thus, sediments are an important pathway for transport and exposure for these contaminants. Data for the WOCE and WOL indicate that large inventories of contaminants reside in the sediments. These inventories reflect the contributions of upgradient contaminant source areas. The primary risk to off-site areas results from the transport of ${ }^{137} \mathrm{Cs}$ in sediments (Blaylock et al. 1991.a). Because sediments are mobilized and transported during storms, we need information for contaminant transport during high flow conditions to track contaminant releases. Further, there is a need to be able to predict contaminant releases during extreme hydrological events (e.g., a storm with a 100-year return frequency) and to incorporate the influences of watershed modification as a result of remedial actions (e.g., capping) and other development on the contaminant releases. Thus, we need to develop and validate models capable of predicting sediment transport at the watershed level that link WAG and non-WAG areas with the Clinch River.

There are large areas of the WAG 2 floodplain for which no data exist. For most other areas of the floodplain, few data for nonradiological contaminants exist. Sediment analyses may be useful for identifying contaminant sources. Data for sediments are also needed to determine the inventories of contaminants in the system as these relate to the potential for contaminant transport off-site and the evaluation of corrective measures.

Surface water hydrology. Hydrologic fluxes drive contaminant fluxes; hence, data for watershed hydrology are needed. Accurate information for surface water discharge is needed to construct reach-by-reach mass balances of contaminant ch ansport needed to quantify fluxes and to identify source areas. Basic information for hydrology (precipitation, evapotranspiration, surface water flow) are needed to, drive models of contaminant transport and to provide a basis for evaluating changes in contaminant movement. An extensive 
watershed hydrology program is managed by the Environmental Restoration Monitoring and Assessment Program (ERMA) and will support WAG 2 activities (Clapp et al. 1991).

Groundwater (subsurface environment). The hydrogeology of the WOC watershed was discussed in Sect. 3.3.3 of ORNL (1990). Existing information suggests that groundwater contamination can be important in localized areas but is not widespread (see ORNL [1990], Sect. 6.3). In the WOC watershed, groundwater elevations (water table) tend to follow surface topography, greater than $95 \%$ of groundwater discharges into surface water prior to leaving the watershed, and no substantial flow leaves the basin as groundwater (Solomon et al. 1991). Additional information for fluxes and flow pathways of deep groundwater are needed to evaluate contaminant fate, transport, and exposure for individual WAGs and for the entire watershed. Because groundwater phenomena occur on a large scale (i.e., greater than a single WAG), aspects of subsurface transport of contaminants benefit from watershedlevel information.

Because virtually all of the groundwater flux in the WOC watershed discharges to surface water prior to leaving the watershed, the identification and monitoring of springs and seeps (groundwater discharge areas) can help to identify, quantify, and track contaminant fluxes. This information is important for evaluating contaminant transport and potential exposure routes for risk assessment, determining the nature and extent of contamination, and designing remedial actions.

The stormflow zone is a shallow zone approximately corresponding to the root zone of the vegetation that is much more permeable than the unsaturated zone (Moore 1988). Stormflow is transient but may be an important pathway for water following precipitation events (Moore 1988; Solomon et al. 1991). The role of the stormflow zone for contaminant transport into WAG 2 needs to be evaluated. An understanding of hydrologic and contaminant fluxes in the stormflow zone and techniques to monitor stormflow zone are needed to ensure that the remedial alternatives selected are appropriate for mitigating contaminant fluxes. Monitoring in WAG 2 can provide information on contaminant fluxes via the stormflow zone and, so, meet the needs of WAG 2 and source WAGs in evaluating contaminant fluxes.

Contaminants moving through the subsurface can diffuse into soil and rock matrices. These contaminated fractures and pore spaces can act as secondary source areas that release large pools of contaminants after the upgradient primary sources (buried wastes) have been remediated. Information on this process is needed to evaluate corrective measures for the ORNL WAGs.

Biota. The Biological Monitoring and Abatement Program (BMAP) provides extensive information for contaminants in aquatic and some terrestrial biota (see ORNL [1990], Sects. 5 and 6). Aquatic biota accumulate ${ }^{137} \mathrm{Cs},{ }^{60} \mathrm{Co},{ }^{90} \mathrm{Sr}$, mercury, PCBs, and chlordane. The influence of sediment contaminants on the aquatic biota needs to be evaluated. Floodplain vegetation is contaminated with radionuclides (primarily ${ }^{3} \mathrm{H},{ }^{90} \mathrm{Sr}$, and ${ }^{99} \mathrm{Tc}$ ); however, few data are available for other contaminants. Resident and migratory waterfowl accumulate contaminants and are a direct pathway of exposure for the public to contaminants from WAG 2. 
Terrestrial biota are important for the movement of contaminants and the potential transfer to humans. Terrestrial biota may also be at direct risk from exposure to contaminants. Data for terrestrial biota are generally needed.

Other sources of information. A number of monitoring programs, assessment programs, as well as RIs are under way in the WOC watershed or directly downgradient (i.e., the Clinch River RI [CRRI]). These programs constitute an important source of information and provide opportunities for collaboration. Interactions with these projects and programs are discussed in Sect. 2.3.1.

\subsection{PURPOSE AND APPROACH}

The purpose of the S\&A Plan is to collect physical data and to collect and analyze environmental samples for physical, chemical, and biological paramters to support the WAG 2 environmental monitoring program and to form a basis for the preliminary components of the formal remedial investigation/feasibility study (RI/FS) for WAG 2. The S\&A plan for the WAG 2 RI project will also make important contributions to the management of remedial activities in the WOC watershed.

The WOC system is important as an iniegrator of contaminant releases from ORNL faci." ies and as a conduit for the transport of contaminants off-site. The S\&A activities support a multimedia environmental monitoring program that will define and track contaminant inputs into WAG 2 from adjacent WAGs. Information for contaminant releases from ORNL WAGs is needed to guide remedial efforts. Because the RI/FS process for the individual ORNL WAGs will not be conducted concurrently, data to rank contaminant sources and to track contaminant releases will not be available to the ORNL ER program. Because WAG 2 encompasses the lower drainage system, contaminants found in WAG 2 and the processes and pathways of contaminant transport in WAG 2 should be important throughout the watershed. Further, because some processes are best addressed at the watershed level (e.g., sediment/contaminant transport, groundwater/groundwater transport) the WAG 2 RI project takes a watershed-level approach where appropriate. Thus, information generated by the WAG 2 multimedia environmental monitoring efforts will transfer to other WAGs in the watershed and facilitate restoration of those areas.

These S\&A activities will address the short-term needs to protect the public and the environment, support other remedial efforts at ORNL, and form a basis for the eventual remediation of WAG 2. Specifically, the S\&A Plan supports the preliminary components of the RI/FS for WAG 2 by (1) providing a preliminary characterization of the nature and extent of contamination in WAG 2, (2) quantifying the risk to human health and the environment resulting from the contamination, (3) identifying operable units, and (4) developing a conceptual model to allow preliminary evaluations of potential corrective measures for the operable units in WAG 2.

As noted, the WOC system is complex, with many facilities and diverse pathways of contaminant transport, and is dynamic, where conditions related to contaminant fluxes are changing. Under these conditions, a complete and exhaustive site characterization and/or efforts to quantify all fluxes via all flow pathways are unreasonable. Instead, we have adopted an approach analogous to the Observational Approach in which we (1) focus on contaminants and pathways of primary concern based on a risk-based preliminary contaminant screening and 
(2) monitor and gather sufficient information for processes controlling or driving contaminant fluxes to construct an appropriate conceptual model for WAG 2. This approach allows the early identification of remedial alternatives and focuses efforts on the gathering of data useful and sufficient for evaluating alternatives to reduce risk to the public and the environment.

Because little information is available for some areas of WAG 2, preliminary scoping-level characterization of these areas is needed. Data from these efforts will be used to update the risk assessment. The multimedia environmental monitoring effort will evolve as new information becomes available and as needs and conditions change. Therefore, the approach will be one that (1) focuses on contaminants and pathways of primary concern for human health and environmental risk, (2) provides scoping and screening-level information needed to update the risk assessment, and (3) evolves to meet changing needs of the ORNL ER Program.

The WOC system has been divided into four reaches for the preliminary risk analysis. The monitoring program will deal with the WOC system on a reach-by-reach basis and will support a mass-balance approach for determining contaminant dynamics for each reach (i.e., input, storage, and release). These reaches will be subdivided as required based on contaminant input and inventory. This approach translates to the identification of operable units to be considered for corrective measures or eliminated from further efforts.

'The plan includes a hydrologic empirical modeling program to predict the movement of contaminants into WAG 2 from adjacent areas, the fate and transport of contaminants within WAG 2, and the transport of materials out of WAG 2 to the Clinch River. Hydrologically-driven contaminant transport models are needed to predict changes in contaminant transport due to natural environmental factors (e.g., precipitation), changing land use in the watershed (e.g., increased paving), and remedial activities (e.g., capping) in upgradient WAGs.

The strategy for the S\&A plan (discussed in Sect. 2.2) focuses initially on radionuclides in aquatic sediments, floodplain soils, and entering the main channels via seeps and tributaries. We focus on radionuclides (specifically ${ }^{137} \mathrm{Cs},{ }^{60} \mathrm{Co},{ }^{3} \mathrm{H}$, and ${ }^{90} \mathrm{Sr}$ ) because radionuclides are major contributors to human health risk and because gamma emitting radionuclides $\left({ }^{137} \mathrm{Cs}\right.$ and ${ }^{60} \mathrm{Co}$ ) can be rapidly surveyed in the field and then measured in the laboratory. Fewer samples will be analyzed for metals, organics, and other radiological contaminants (e.g., transuranics, $\left.{ }^{99} \mathrm{Tc},{ }^{152,154} \mathrm{Eu}\right)$. The goal of the initial stages of the S\&A plan is to scope or screen for contaminants in areas where few data exist, to begin to develop contaminant inventories, and to determine if data for radionuclides can be used to guide future sampling for other contaminants of concern. The ability to use radionuclide data to guide future sampling efforts will greatly facilitate the S\&A activities.

Data from surveys and initial sampling efforts will be used to stratify the system for later sampling efforts, to estimate the initial level of variance for parameters of interest, and to determine the cost required to reduce the uncertainty in the estimates of risk and to determine that risk threshold levels are not exceeded.

The initial focus is on sediments, seeps and tributaries. Sediments are the primary pool for all contaminants, except ${ }^{3} \mathrm{H}$, in WAG 2. Exposure to radionuclides in sediments is the primary contributor to potential human health risk, and key contaminants are transported off-site with sediments during high discharge events. Seeps and tributaries are important as 
pathways for contaminant input from adjacent WAGs into WAG 2 and as connections between contaminated groundwater and surface water in WAG 2.

Biota can be useful indicators of contaminant availability in the system. We will gather preliminary data for contaminants in biota since biota accumulate contaminants (e.g., PCBs) that are important for human health risk, and data are needed to update estimates of ecological risk.

Groundwater is not believed to be a major contributor to risk; thus, initial efforts on groundwater focus on (1) the evaluation of incoming data from wells located on the perimeters of the ORNL WAGs to identify contaminant sources and pathways and (2) the development of a conceptual model for groundwater in the WOC watershed. Data provided by the seep sampling program will support the groundwater efforts.

A diverse array of monitoring activities mandated by ORNL's NPDES permit and DOE orders provides an important source of information for the WAG 2 investigation and offers opportunities for collaboration on data collection. Activities related to environmental restoration [RIs, Resource Recovery and Conservation Act (RCRA) facility investigations (RFIs), and special studies] under way in the WOC watershed and the Clinch River (administered by the Off-Site ER Program) by necessity are linked to the WAG 2 investigations. These projects and programs provide important support for the S\&A efforts at WAG 2. We have taken steps to develop operational links with these programs, just as the ER Programs of DOE and Martin Marietta Energy Systems, Inc. (Energy Systems) provide administrative linkages. The formalized links with these other programs that support the S\&A plan are discussed in Sect. 2.3.2 and are noted appropriately elsewhere.

\subsection{OBJECTIVES}

The general objectives of the S\&A plan are to provide a multimedia environmental monitoring and characterization program to (1) define and monitor the input of contaminants to WAG 2 from other WAGs; (2) support a mass balance approach to determining sources, sinks, and transport of contaminants in WAG 2 based on hydrologic fluxes; (3) establish the basis for documenting long-term trends in contaminant pools and fluxes; and (4) develop models that predict potential contaminant releases under future conditions.

Specific objectives identified for the first 2 years of effort are outlined here.

\section{A. SEDIMENT SAMPLING PLAN}

1. Conduct floodplain radiological walkover and stream sediment surveys.
a. Radiological walkover.
b. Floodplain soil sampling.
c. Preliminary stream sediment sampling.

2. Sample contaminant transport during storms.

3. Quantify and track contaminant inventories in stream sediments and identify contaminant sources.

a. Distribution and inventory of radiological contaminants in stream sediments. 
b. Stream gravel survey program.

4. Develop models to predict sediment transport for the WOC watershed.

a. Develop a quantitative data base for evaluating phenomena observed (stagedischarge-sediment flux) during high-discharge events.

b. Predict contaminant transport under future conditions.

c. Provide estimates of uncertainty in model results.

d. Link models for sediment transport in the WOC watershed with similar efforts for the Clinch River RI.

\section{B. SURFACE WATER SAMPLING PLAN}

1. Determine contaminant fluxes in surface water.

2. Identify tributaries that contribute significantly to contaminant flux within WAG 2.

3. Monitor tributaries to quantify contaminant fluxes.

4. Identify areas (discrete or diffuse) of groundwater discharge that contribute significantly to contaminant flux in the streams.

5. Monitor seeps to quantify contaminant fluxes.

\section{GROUNDWATER SAMPLING PLAN}

1. Incorporate existing and incoming data into a dataset to serve as a reference for testing hypotheses and interpreting groundwater flow and geochemical data.

2. Conduct statistical pattern recognition analysis of groundwater data.

3. Evaluate the importance of groundwater flow in migration of contaminants from adjacent WAGs.

4. Cooperate with ongoing investigations to evaluate the role of the stormflow zone in contaminant transport in WAG 5 and to evaluate the importance of matrix diffusion and the creation of secondary source areas for contaminant release from WAG 5 to WAG 2.

\section{ECOLOGICAL ASSESSMENT SAMPLING PLAN}

1. Expand an existing model of the WAG 2 ecosystem to incorporate organisms at risk and important pathways to humans.

2. Integrate BMAP data and data from other WAGs to quantify contaminant movement through the food chains.

3. Obtain data on contaminant levels in organisms identified as important but not covered by BMAP or other WAGs programs.

4. Periodcially update ecological assessment. 


\title{
2. SCOPE AND IMPLEMENTATION
}

\author{
J. A. Watts, H. L. Boston, D. J. Downing, G. W. Suter, D. E. Miller, and V. Chidambariah
}

\section{CONTAMINANTS AND PATHWAYS OF CONCERN}

\subsubsection{Human Health Risk}

Information for contaminants of concern and pathways of transport and exposure for human health risk derive from the preliminary contaminant screening for WAG 2 (Blaylock et al. 1991b) and from the interim site characterization and contaminant screening for the WOCE (Blaylock et al. 1991a) that was conducted earlier as part of a time-critical removal action required by the Comprehensive Environmental Response, Compensation, and Liability Act (CERCLA). We also employ data for applicable or relevant and appropriate requirements (ARARs), contaminant screening for adjacent WAGs, and other siudies and monitoring efforts that provide information for contaminants and transport for the WOC watershed (see Clapp et al. 1991) to develop a list of contaminants of concern for WAG 2. The screening analyses for WAG 2 and the WOCE component of WAG 2 considered carcinogens and noncarcinogens in water, soil and sediment, and biota for an occupant of WAG 2 under current conditions and for a hypothetical hunter/fisherman intruder scenario. For the WAG 2 screening, the area upgradient of the embayment was divided into three reaches: Reach 1, the WOC arm (above the confluence with Melton Branch); Reach 2, the Melton Branch arm (above the confluence with WOC); and Rcach 3, the WOL reash which included the lake and its floodplain (Fig. 2.1). Screening analyses were conducted for the data base of detected contaminants and the nondetectable data base (i.e., contaminants which were not present at concentrations above the detection limit of the analytical method used and so were reported as "less than" values).

The approach taken in the screening exercises was similar to Hoffman et al. (1990), employing both conservative and nonconservative procedures. The conservative procedure uses higher than expected (i.e., EPA standard) exposure rates and so is not likely to underestimate maximum exposure. The conservative screening provides an upper bound of potential exposure. Altematively, a nonconservative procedure uses more realistic estimates of exposure (i.e., lower than standard EPA exposure rates) and thus should not substantially overestimate the maximum exposure to an individual in the area. The risis is then calculated based on the exposure scenario, the estimate of concentration in the media (median or upper 95\% estimate for the nonconservative or conservative procedure, respectively, and the EPA approved or suggesisd slope factor for carcinogens or reference dose factor (RfD) for noncarcinogens. For the nonconservative screening procedure, contaminants with an excess lifetime cancer rick of $>10^{-4}$ are "definitely high priority" and warrant immediate consideration. A noncarcinogenic hazard index (estimate of daily ingestion or inhalation, divided by the RfD) that is $>1$ is considered to be "high priority" and warrants immediate consideration.

At this stage, we will focus on results of the nonconservative screening. Details of the results for WAG 2 are presented in Blaylock et al. (1991b). All three reaches had screening indices $>10^{-4}$ for carcinogens and $>1.0$ for noncarcinogens. Thus, all three reaches are identified as requiring immediate consideration. Similar findings for the WOCE (Blaylock et al. 1991a) have resulted in a CERCLA time-critical removal action to control the resuspension and downstream transport of sediments. 


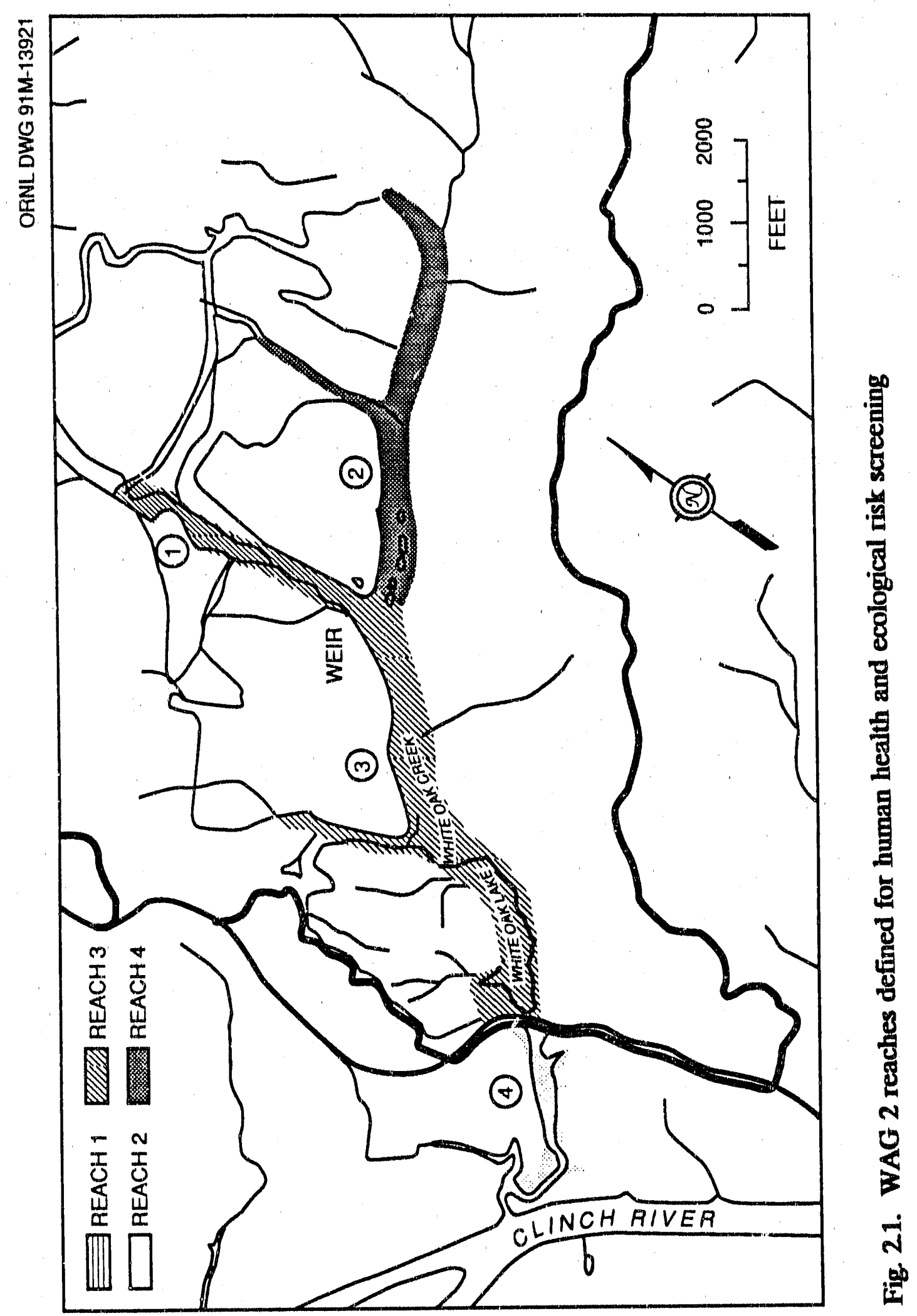


Data for sediment contaminants were limited to selected areas of WAG 2 and were much more extensive for radionuclides than for metal or organic contaminants. External exposure radionuclides in sediments were definitely high priority (i.e., excess cancer risk $>10^{-4}$ ) for all reaches. In general, ${ }^{137} \mathrm{Cs}$ contributed greatest to risk, except in the Melton Branch arm of the system (Reach 2), where the facilities acting as the major ${ }^{60} \mathrm{Co}$ sources and less important ${ }^{137} \mathrm{Cs}$ sources occur. Overall, for the external exposure pathway, ${ }^{137} \mathrm{Cs}$ and ${ }^{60} \mathrm{Co}$ were "high priority, ${ }^{1}$ while ${ }^{152} \mathrm{Eu},{ }^{134} \mathrm{Cs}$, and ${ }^{154} \mathrm{Eu}$ will require additional consideration. Limited data were available for ${ }^{241} \mathrm{Am},{ }^{24} \mathrm{Cm},{ }^{238,239} \mathrm{Pu}$; thus, these contaminants should be considered for further sampling and analysis.

The sediment-ingestion pathway had no contaminants of high priority; however, arsenic in Reach 1 and ${ }^{137} \mathrm{Cs}$ for the total pathway required additional information.

PCBs in fish were "high priority" in Reaches 1 and 3 and warranted additional information in Reach 2. Additional information is required for ${ }^{137} \mathrm{Cs}$ in all reaches and mercury in Reach 1 (i.e., hazard index $>0.1$ ).

Although surface water in WAG 2 generally meets ORNL's NPDES permit requirements, the screening indicated that additional data are needed for ${ }^{90} \mathrm{Sr},{ }^{3} \mathrm{H}$, and $\mathrm{PCBs}$ in all reaches. Additional data are also needed for ${ }^{152} \mathrm{Eu}$ and ${ }^{154} \mathrm{Eu}$ (Reach 2 only), ${ }^{137} \mathrm{Cs}$ (Reaches 1 and 3 only), ${ }^{235} \mathrm{U}$ (Reach 3 only), and for dichlorobromomethane (Reach 1 only). Arsenic and thallium were "high priority" in all reaches; however, this result was based on few analyses for thallium and a low proportion of samples in which arsenic was detected.

No contaminants in WAG 2 groundwater screened as "high priority," with the possible exception of lead. For a number of contaminants (beryllium, arsenic, antimony, ${ }^{3} \mathrm{H},{ }^{236} \mathrm{Ra}$, ${ }^{244} \mathrm{Ra},{ }^{230} \mathrm{Th},{ }^{234} \mathrm{Th},{ }^{211} \mathrm{Am},{ }^{234} \mathrm{U}$, and ${ }^{238} \mathrm{U}$ ), the screening indicated that additional data were required. For several of these contaminants, the screening was based on few analytical reports (e.g., one report from a total of four analyses). Although these data indicate that the contaminant is present they do not provide reliable estimates of concentrations for screening. Data for organic contaminants in WAG 2 groundwater were generally not reliable for screening; thus, additional data are needed.

Most of the reports for organic compounds in the data base were below the analytical detection limit. A screening of the nondetect data base identified several organic compounds as "high priority" (i.e., excess cancer risk $>10^{-4}$ ) for sediment ingestion (e.g., benzidine, $N$. Nitrosodimethylamine). A larger group of organics from the nondetect data base were identified as "high priority," based on conservative screening.

A realistic intruder scenario considered a hunter/fisherman illegally entering WAG 2 for fishing ten times per year, $4 \mathrm{~h}$ per trip, for 10 years, and for hunting $10 \mathrm{~d}$ per year for $6 \mathrm{~h}$ each trip, for 10 years. The pathways considered were consumption of fish, waterfowl, and deer, and the external exposure from radionuclides in soil and shoreline sediments (Blaylock et al. 1991b). Based only on the contaminants detected (i.e., not including the nondetect data base), the total screening indices for all reaches were $\geq 10^{-4}$. Reach 1 had a higher screening index than Reach $2\left(1 \times 10^{-3}\right.$ and $6 \times 10^{-4}$, respectiveiy), and ${ }^{137} \mathrm{Cs}$ in fish was the primary contributor to the total index. $\mathrm{PCBs}$ in fish were also high priority in all reaches. For Reach 3 , PCBs in fish were the contaminant of greatest concern, followed by ${ }^{137} \mathrm{Cs}$ in fish, for a total screening index of $8 \times 10^{-3}$. 
The screening for the WOCE (Blaylock et al., 1991a) was based on a much more extensive data base collected during 1990 . That screening found that exposure to ${ }^{137} \mathrm{Cs}$ in sediments and ingestion of PCBs in fish constituted the majority of the risk to a potential occupant or an illegal intruder. Other radiological contaminants of concern included ${ }^{3} \mathrm{H},{ }^{90} \mathrm{Sr}$, ${ }^{60} \mathrm{Co}$, and possibly ${ }^{152} \mathrm{Eu},{ }^{154} \mathrm{Eu},{ }^{259240} \mathrm{Pu},{ }^{244} \mathrm{Cm}$, and ${ }^{99} \mathrm{Tc}$. For nonradiological contaminants in sediment, fish, and water, PCBs and arsenic probably were important, whereas further information on chlordane, $\mathrm{Be}, \mathrm{Cr}, \mathrm{Ag}, \mathrm{Se}$, and $\mathrm{Pb}$ is required. Screening for using detection limits for nondetects identified 16 organic carcinogens as being definitely high priority.

These findings are being given further consideration to determine which are probably artifacts and which are reliable. In general, additional data are required for metals, radionuclides, and organics in all media. The effort expended on obtaining these data, however, should be guided by the results and interpretation of the contaminant screening and other available information (e.g., data from other WAGs and historical use of compounds of interest).

\subsection{Ecological risk}

A screening assessment of ecological effects in WAG 2 has been conducted concurrently with this report (Blaylock et al. 1991b). This assessment considered three lines of evidence concerning the risks to nonhuman organisms posed by contaminants in WAG 2: biological surveys, toxicity tests of ambient media, and exposure/response analysis for measured contaminant concentrations. The biological survey data indicate that aquatic effects are not severe in that a diverse and productive aquatic community is found in WAG 2. However, comparison of the aquatic biota to those of reference streams indicates that the composition of the benthic invertebrate community may be modified and fish reproduction may be disrupted. Biological survey data are not available for terrestrial biota.

Recent toxicity tests of water from WAG 2 do not indicate toxicity to Ceriodaphnia dubia or to larval fathead minnows in 7 day bioassays. No toxicity tests have been performed on sediments or soils.

Comparison of media concentrations with toxicological benchmarks produced ambiguous results because of the large number of chemicals that were not detected but had limits of detection higher than potentially toxic concentrations. Mercury and PCBs were found at potentially toxic concentrations in both water and sediments in all reaches. Aluminum, cadmium, chromium, copper, and lead exceeded national ambient water quality criteria and state standards, and twelve other metals exceeded potentially toxic concentrations. Of the chemicals that had been detected in sediments and for which available concentrations could be estimated, barium, cobalt, mercury, silver, zinc, benzene, di-n-butyl phthalate, methylene chloride, and PCBs are potentially toxic to benthic organisms. Selenium and possibly cadmium were found in fish flesh at concentrations indicative of toxic effects. Mercury and PCBs occurred in fish flesh at concentrations that are potentially toxic to piscivorous wildlife based on dietary toxicity data, and many others occurred at concentrations that would exceed the reference dose for human health effects when wildlife consumption rates were used. No analyses could be performed for toxic effects on terrestrial organisms other than piscivorous wildlife. 
One can conclude from this evidence that ecotoxicological effects may be occurring in W.1G 2, but they are not as severe as would be suggested by the exposure/response analysis using the reported chemical concentrations. This discrepancy is due in part to the conservatism of the screening rriteria, but the authors believe that the principal factor is the inappropriateness of many of the analyses as estimators of bioavailable concentrations. Therefore, future activities should focus on estimation of actual exposure levels. In addition, chemical and biological data are needed from terrestrial portions of WAG 2. Future assessments will focus on improving the relevance of exposure-response estimates to conditions in WAG 2 and will continue to attempt to reconcile the three lines of evidence for estimating ecological effects (i.e., biological surveys, toxicity tests, and contaminant concentrations).

\subsection{Summary of data needs}

For human health risk, external exposure to radionuclides $\left({ }^{137} \mathrm{Cs}\right.$ and $\left.{ }^{60} \mathrm{Co}\right)$ in sediments, PCBs in fish, and arsenic and thallium in water were screened as high priority requiring immediate consideration for remedial action. Lead in groundwater is a possible addition to the list of high priority contaminants. Contaminants that screened at slightly lower risk, for which additional data are required are: organic compounds, mercury, and ${ }^{137} \mathrm{Cs}$ in fish; ${ }^{137} \mathrm{Cs}$, ${ }^{90} \mathrm{Sr},{ }^{3} \mathrm{H},{ }^{152} \mathrm{Eu},{ }^{154} \mathrm{Eu}$, and ${ }^{23} \mathrm{U}$ in water; and ${ }^{152} \mathrm{Eu}$ and ${ }^{154} \mathrm{Eu}$ in sediments.

A lack of sufficient data made the evaluation of organic contaminants in all media and transuranics in several media suspect.

Because institutional controls are in place, these risks are potential risks because the public is not now exposed on a routine basis. There are two exceptions, however, (1) the off-site transport of contaminated sediments and (2) resident and migratory waterfowl. Waterfowl accumulate radionuclides, organic compounds, and metals while feeding on White Oak Lake (Loar 1991). These waterfowl represent a active pathway for potential public exposure to on-site contaminants. Waterfowl in WAG 2 are being investigated by the BMAP, and the data collected will be used to evaluate potential risk to the public.

Data available for the ecological risk screening suggested that severe effects on aquatic biota are not occurring; however, a number of compounds (notably mercury and PCBs) were present in sediments and water at concentrations that are potentially toxic to biota. Additional analyses with lower detection limits will be required to evaluate the potential for toxic effects on aquatic organisms. A general need exists for survey data for terrestrial biota. Among terrestrial biota, piscivorous (fish eating) wildlife may be at risk.

Potentially significant risks were found for organic contaminants that were not detected but were screened at a concentration equal to the detection limit for both the human health and ecological risk screenings (see Blaylock et al. 1991b). This is problematic because in many cases there is no reason to suspect that a given compound is present. A rationale for dealing with nondetects is provided in Sect. 2.5.4.

\subsection{STRATEGY AND SCOPE}

A col ieptual model of WAG 2 (Fig. 2.2) has been developed based on existing information and results of the contaminant screening. The S\&A efforts during the early 


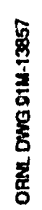

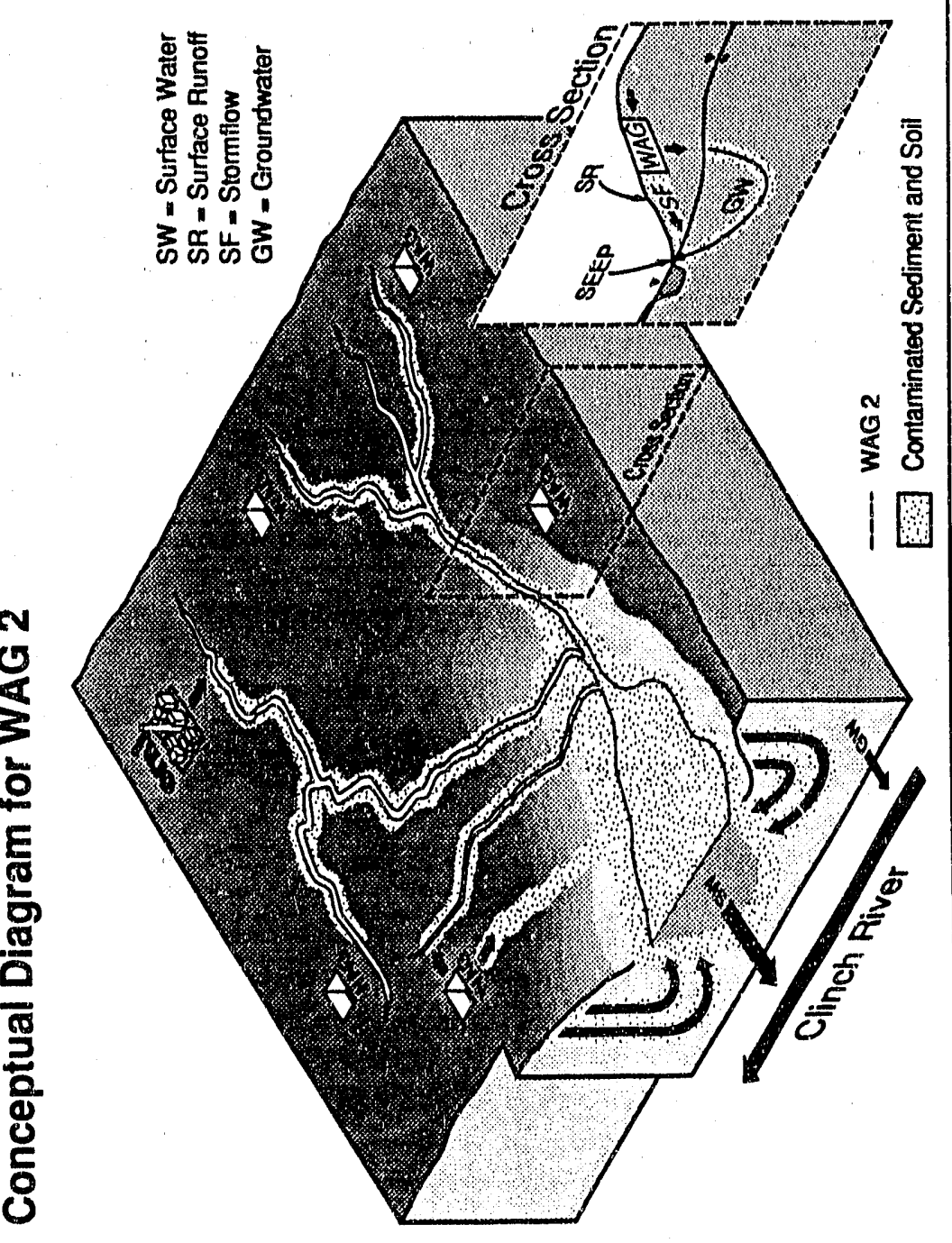

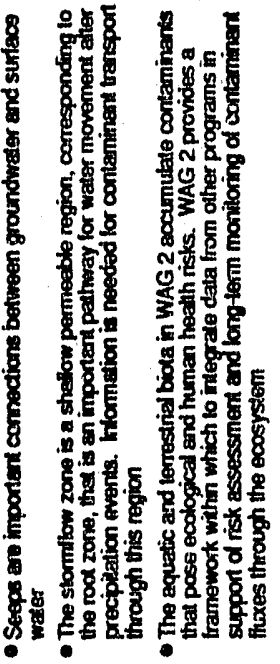

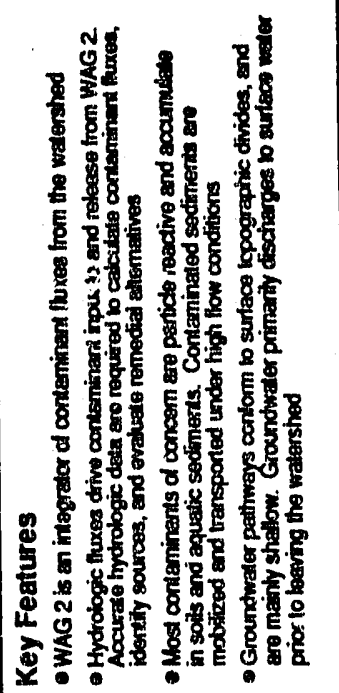

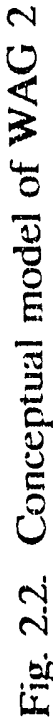


stages of the WAG 2 RI will focus on implementation of a multimedia monitoring program to be conducted for the next 10 years or more while upgradient WAGs are remediated. During this period, in addition to identifying contaminant sources, quantifying contaminant releases, and evaluating pathways of contaminant movement, we will continue to update the risk assessment by screening for additional contaminants of concern and incorporating new information for parameters, areas, and components where few or no data currently exist. The preliminary activities or scope of the WAG 2 RI S\&A plan are summarized below and presented in detail in Chapters 3-6.

Sediment sampling plan. Aquatic sediments and floodplain soils are the primary pools for contaminants in WAG 2. Exposure to radionuclides in sediment represent the greatest potential risk for future occupants of WAG 2, and the transport of contaminated sediments out of the WOC system is the primary concern for risk to off-site areas. The sediment sampling plan will be the primary focus of activities during the first 2 years.

Data for the distribution and concentration of key contaminants are lacking for many areas of the aquatic systems and floodplain in WAG 2. The sediments and floodplain of the WOCE have recently been well characterized (Blaylock 1991a), and there is good preliminary information for contaminants in WOL sediments. Thus, initial efforts will address the remaining 80 to 100 acres of WAG 2 for which few data are available. The first objective of the sediment sampling plan is to conduct a radiological walkover of the WAG 2 floodplain. This will provide initial information for the presence of areas in need of interim corrective measures and will form a basis for the stratification of the floodplain for later more intensive sampling. A preliminary sampling of floodplain soils and stream sediments is included in this objective. The data provided will be used to update information for contaminants present in various reaches and will provide an initial estimate of variance to determine additional sampling needed to improve estimates of risk.

The second objective is to sample contaminant transport during storms at key locations in the watershed. These data will be used to (1) quantify sediment associated contaminants moving through specific reaches of WAG 2 to aid in the identification and quantification of contaminants sources, (2) quantify contaminants released from the watershed during high-flow events, and (3) support the development of a model to predict the transport of contaminated sediments out of WAG 2. This activity is support by the ERMA watershed hydrology program (Clapp et al. 1991).

The third objective is to provide an initial quantification of the inventory of major contaminants in the stream sediments of WAG 2. As noted, good preliminary data exist for sediments in WOL and the WOCE. An inventory of contaminants in stream sediments is important for tracking contaminant inputs, predicting contaminant releases (i.e., data needed for modeling), and identifying remedial alternatives. A stream gravel survey program will be initiated as part of this effort that will help to identify and track sources of contaminant releases to surface water.

The fourth objective is the development of a model capable of predicting sediment transport (and contaminant transport) out of the WOC watershed during extreme hydrologic events and following changes in the watershed as a result of remedial actions or other development. This component builds on the data collected by the other components of the sediment sampling plan and is linked with modeling efforts in upgradient WAGs with active RI/FSs and with the CRRI to provide a nested approach for predicting contaminant transport 
from ORNL WAGs to off-site areas. This information is needed to evaluate potential risks to off-site areas and so to guide remedial actions.

Surface water. Surface water is the primary transport pathway for particle associated and dissolved contaminants out of the WOC watershed. The transport of sediment-associated contaminants is addressed in the sediment sampling plans, and thus these components are being closely coordinated. There are a number of dissolved contaminants of concern in surface water (notably ${ }^{3} \mathrm{H},{ }^{90} \mathrm{Sr}, \mathrm{PCBs}$, and possibly arsenic and thallium). Under baseflow conditions the transport of dissolved contaminants can be quantified for the major reaches of WAG 2 by data provided by two existing monitoring programs at ORNL that collect data for compliance with state and federal regulations or for compliance with DOE orders. The first objective of the surface water sampling plan is to use data generated by the compliancedriven monitoring programs to quantify contaminant fluxes (under non-storm conditions) from the major reaches of WAG 2. These efforts will be augmented in year 2 to include organic contaminants. This component supports efforts to identify sources and fluxes of contaminants into WAG 2 via seeps and tributaries.

The second objective of the surface water sampling program is to identify seeps and tributaries that are responsible for contaminant fluxes to the main channels of WAG 2 . Because greater than $95 \%$ of groundwater discharges into surface water prior to leaving the WOC watershed, seeps (connections between groundwater and surface water) are useful for evaluating fluxes of contaminated groundwater. This component will coordinate with the groundwater efforts, and the compliance monitoring at the major weirs to (1) identify seeps and tributaries responsible for contaminant fluxes to WAG 2, (2) determine sources of those contaminants, (3) develop a progam to track contaminant inputs, and (4) provide information to determine if interim corrective measures are appropriate. The tributary component will be supported by ERMA and will be linked to the WAG 2 sediment sampling efforts to evaluate contaminant transport during high-flow events.

Groundwater. Although there is significant contamination of groundwater in some areas of WAG 2, the contamination is apparently not widespread. Additional information is needed for all classes of contaminants in groundwater. The key questions for the groundwater component of the S\&A plan are to determine (1) the distribution of contaminants in groundwater (which contaminants, which groundwater masses), (2) the sources of those contaminants, (3) the transport of contaminated groundwater, and (4) to provide data and parameter estimates for the assessment of remedial alternatives. Water quality wells (173 total) have been installed on the perimeters of eleven ORNL WAGs. Beginning in 1991, these wells are being sampled quarterly for an extensive suite of parameters. Those data along with data available from the hundreds of wells located within the WOC watershed will be used to address a series of preliminary objectives. The groundwater component, therefore, initially will focus on the review and analysis of existing and newly acquired data.

The first objective is to construct a data set that will include information about geologic formation, monitored zone, hydraulic conductivity, water level, and other parameters to serve as a reference for hypothesis testing and groundwater flow and geochemical data interpretation.

The second objective is to evaluate data for groundwater geochemistry to develop a picture of the nature and extent of contaminant transport in groundwater. Statistical pattern recognition analysis will be used to evaluate the data. 
The third objective is to conduct a series of hydrogeologic investigations in conjunction with and supported by the ERMA program (Clapp et al. 1991) to evaluate the role of groundwater flow in the migration of contaminants from adjacent WAGs through WAG 2 to streams. These efforts focus on measurement of water levels and physicochemical data for selected wells, identification of discrete flow zones, evaluation of contaminant distributions relative to hydraulic gradients, and coordination with seep sampling efforts to evaluate groundwater flow paths.

Biota. The fish ingestion pathway is second to sediments in potential risk to human health, as a result of PCBs, radionuclides, and metals in fish. Other biota (e.g., deer, wild turkeys) are also potential pathways of contaminant transfer to humans. As noted in Sect. 2.1.2, although severe ecological effects are not apparent in WAG 2, additional data are needed for contaminants and biota because (1) several contaminants are present at concentrations potentially toxic to biota or chemicals that were not detected had detection limits that were higher than levels at which toxic effects can occur, (2) there are few data for terrestrial organisms, and (3) some organisms may be useful indicators of contaminant levels or availability in WAG 2. An ecological assessment plan is presented that is based on (1) collection of data by the BMAP (Loar 1991), (2) biological sampling to support remedial investigations in other WAGs, and (3) biological monitoring in WAG 2. The role of WAG 2 as an integrator of contaminants and a focal point for coordination and identification of data needs is emphasized for this component.

The first objective is to develop a model of contaminant flow to identify organisms at risk and important pathways to humans. This model will be useful for predicting effects of changes in contaminant input or availability in the system.

The second objective is to coordinate efforts of the BMAP and biological sampling programs in specific WAGs to provide an integrated long-term monitoring program to measure impacts of changes in contaminant inputs to the system. This objective includes a survey of threatened and endangered species in WAG 2.

The third objective is to obtain data for organisms at risk (e.g., mink) that are not being obtained by other programs.

The fourth objective is to evaluate information collected by all biological sampling efforts to update the screening-level ecological assessment and to identify the need for additional data. This activity will require close coordination with BMAP.

Specific objectives of this plan. The specific objectives of the preliminary stages of the S\&A plan are presented in Sect. 1.4. These objectives reflect an initial emphasis on three components.

1. Scoping and screening studies to form a basis for statistical design of sampling and monitoring programs. Basic information is needed for the distribution of contaminants (e.g., occurrence of radionuclides with other contaminants).

2 Implementation of the monitoring and tracking efforts. We need to locate seeps carrying significant contaminants and determine how best to monitor these. We need to determine the distribution of sediment contaminants and develop/evaluate our methods for sampling suspended sediments during storms. We need to evaluate data for 
groundwater to identify pathways of groundwater fluxes and identify sources of contaminants. Data for biota will largely derive from other projects (e.g., BMAP and WAG 5 RI).

3. Collecting information for components and contaminants for which few data exist to update the preliminary evaluation of human health and environmental risk.

As noted in Sect. 1.3, our approach will focus on those contaminants and pathways that are most significant for human health and environmental risk (on-site and off-site) and on developing a conceptual model for contaminant fate and transport of contaminants for WAG 2.

The S\&A plan, therefore, initially emphasizes radionuclides because (1) they are major contributors to human health risk, (2) gamma-emitting radionuclides can be rapidly surveyed in the field and measured in the laboratory, and (3) information on radionuclides should provide insight into the behavior of other contaminants. Data on the fate and transport of water-soluble radionuclides (e.g., ${ }^{3} \mathrm{H}$ and ${ }^{90} \mathrm{Sr}$ ) should be generally applicable to other watersoluble contaminants (e.g., volatile organics); similarly, data for particle-reactive radionuclides (e.g., ${ }^{137} \mathrm{Cs}$ and ${ }^{60} \mathrm{Co}$ ) should be generally applicable to particle-reactive contaminants (e.g., most metals and semivolatile organics). Thus, the great majority of samples will be analyzed only for key radionuclides $\left({ }^{3} \mathrm{H},{ }^{90} \mathrm{Sr}\right.$, and gamma emitters), while a fewer number of samples, selected on the basis of location or information on radionuclides, will also be analyzed for metals, organics, and other radionuclides (e.g., transuranics). The samples analyzed for metals, organic contaminants, and other radionuclides will provide preliminary information for the concentrations and variance in the distributions of those contaminants and will allow us to determine if their distributions are well correlated with those for radionuclides. These data will be used to develop the next and more intensive round of sampling and analyses.

At this preliminary stage, our efforts on nonradiological contaminants largely focus on identifying those pathways and areas where contaminants are most likely to be found, rather than conducting an exhaustive characterization and inventory of all contaminants in WAG 2. However, because PCBs and chlordane are known to accumulate in the aquatic biota in WAG 2 and because PCBs in fish are important contributors to potential human health risk, we will emphasize these organics above other organic compounds during the early stages of the RI. Focusing primarily on these two organics will allow us to better evaluate the potential risk associated with these compounds and will provide information useful for guiding sampling for other organic compounds in the future.

This approach has been adopted because it is most likely to provide the greatest return for the initial efforts invested. This approach meets the needs of the WAG 2 monitoring efforts, supports other ER activities in the WOC watershed, and positions us for the eventual remediation of WAG 2.

\section{S\&A ORGANIZATION AND IMPLEMENTATION}

\subsection{External Data Sources}

As a result of DOE orders, EPA and TDEC regulations, and basic research needs, a number of monitoring and assessment programs are underway in WOC watershed, including 
active RI/RFIs on WAGs 1,5 , and 6 . The Clinch River is the primary receptor of contaminants leaving the WOC watershed via WAG 2, and the Clinch River (off-site) RI is collecting data in areas downgradient of WAG 2. [At this time the Clinch River RI is responsible for monitoring during the construction of a coffer-cell dam at the mouth of the embayment to control the release of contaminated sediments (Blaylock et al. 1991a)]. These activities provide potentially important sources of information to the WAG 2 monitoring program, as well as opportunities for collaboration.

The primary monitoring and evaluation efforts underway in and downgradient of the WOC watershed are shown in Fig. 2.3 and briefly described in Table 2.1. The WAG 2 RI project has assigned an extra-project coordinator who has responsibility for seeking and facilitating interactions and collaboration between the WAG 2 project and other activities in the watershed. We have relied largely on information collected by other efforts in the preliminary contaminant screening for WAG 2 (Blaylock et al. 1991b). Collaboration is possible whenever the activities of others meet the data collection QA criteria of the WAG 2 RI project. Documenting these interactions and obtaining QA records is discussed in the QA section (Sect. 2.5) of this document.

A watershed hydrology program (Clapp et al. 1991) provides data for precipitation, evapotranspiration, and streamflow at numerous locations in the watershed. Those data are critical to the WAG 2 project in order to determine mass balances for contaminant fluxes and to drive models. Members of the watershed hydrology group have been incorporated into the WAG 2 RI project (surface water hydrology group) to provide expertise and to facilitate WAG 2 efforts. The ORNL Environmental Surveillance Program (ESP) conducts DOE- and NPDES-mandated surface water monitoring at several key locations in the watershed. The ESP will provide much of the baseflow si!rface water quality information. Again, personnel from ESP have been incorporated into the WAG 2 project. We are collaborating with other projects in several other key areas; instances of interaction with other projects and programs are noted in the following chapters as appropriate.

We are also actively collaborating with the active RI or RFI projects (WAGs 1, 5, and 6) and with the CRRI on data collection, models of contaminant transport, and other areas. Interaction and collaboration with other RI/RFI/feasibility studies (FSs) are especially important to ensure a consistency of approach and mutual data useability across the watershed.

The ORNL ER Program has recently implemented the ERMA Program, whose mission is to facilitate ORNL ER activities by providing coordination among ER activities and coordinating ER activities with activities of other projects in the WOC watershed, assessing trends in data related to contaminant behavior and identification of additional data needs, monitoring watershed hydrology, and conducting directed studies to support ER activities. The WAG 2 efforts contribute to ERMA, and in turn, ERMA is an important source of information for the WAG 2 project.

The BMAP has been monitoring radionuclide and some nonradioactive contaminants in aquatic biota from all reaches of WOC and WOL since 1986 (Loar 1987) in support of NPDES permit requirements. Extensive data are available on contaminants that bioaccumulate in fish and clams and on the results of ambient toxicity testing at several locations (Loar 1987, 1988, 1989, 1990,1991). In addition, the BMAP has collected data on contaminant levels in waterfowl and selected terrestrial species. An important component of 


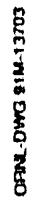

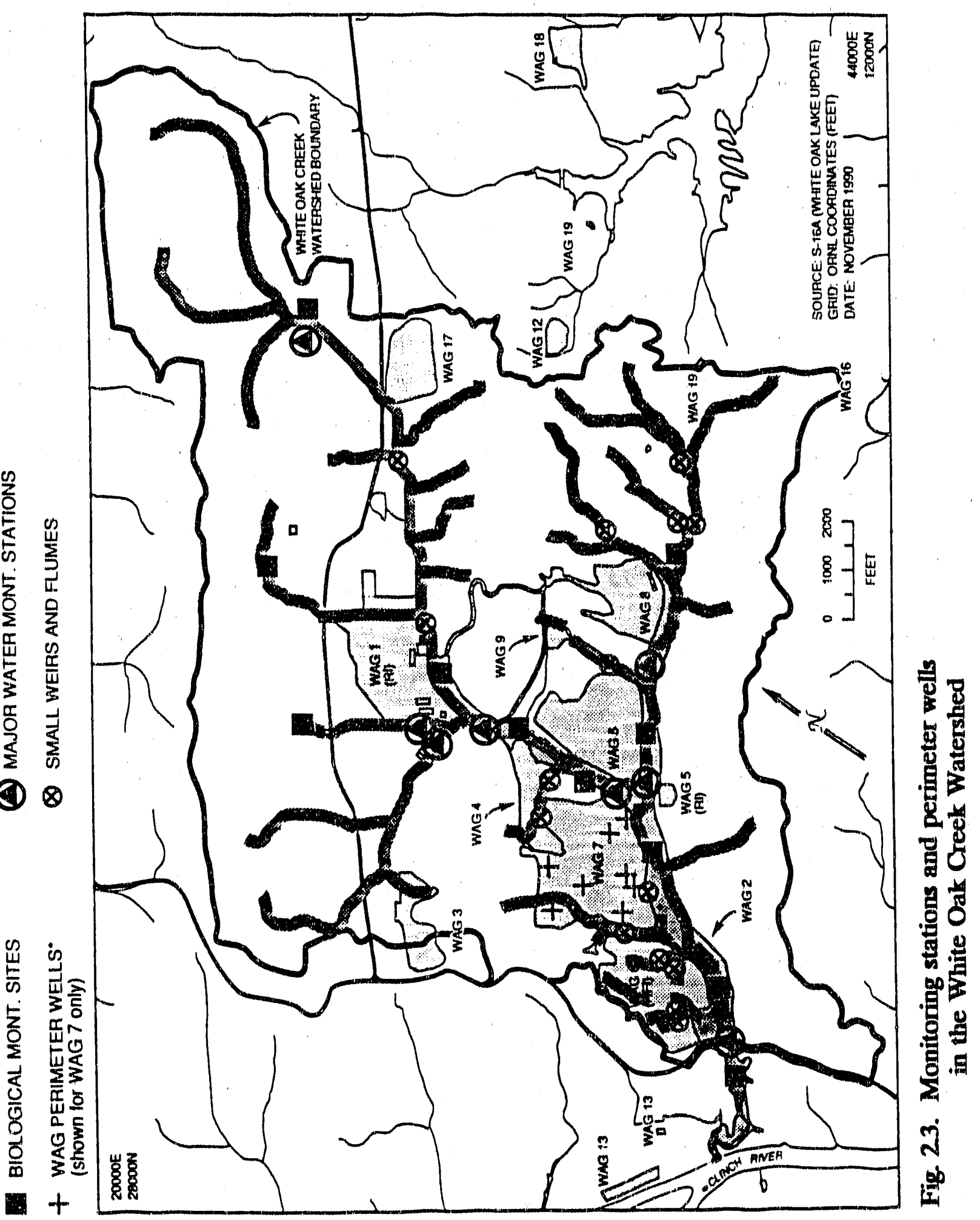


Table 2.1. Monitoring and Investigations that support the WAG 2 RI Project

\section{Surface Water:}

1. The USGS operates nine surface water discharge monitoring stations in the White Oak Creek Watershed (see Table 3,8 in WAG 2 RI).

2. The ORNL ESP operates seven surface water discharge monitoring stations on main branches of the WOC drainage system. Weekly to monthly measurements of metals, radionuclides, some organics, and other water quality parameters are collected at nine stations as part of ORNL's NPDES permit and as mandated by DOE orders. On occasion other parameters (e.g., PCBs in sediments are measured). See Sect. 4 of the WAG 2 RI Plan.

3. ESD Watershed Hydrology group also measures surface water discharge at ten stations (several of the stations coincide with and support the ESP efforts). The ESD group is currently reinstrumenting four additional stations, and plans to reactivate several other stations for sampling of tributaries as part of the ERMA (Clapp et al. 1991). This group also collects data for precipitation and evapotranspiration at five location in the WOC (Borders et al., 1991).

4. ESD conducts the Active Sites Environmental Monitoring Program (ASEMP) which monitors surface water to ensure that radionuclides are not escaping from low level waste disposal sites as mandated by DOE Order 5820.2A (Ashwood et al. 1990 a,b). ASEMP generates water quality and radionuclide data for several sites in WAGs 5 and 6 that drain into WAG 2.

\section{Groundwater.}

1. The ESP section monitors groundwater quality as part of several programs coordinated by the ORNL groundwater coordinator. A DOE Environmental Surveillance Monitoring Program provides an integrating function for RCRA and Remedial Action associated monitoring. RCRA Monitoring is conducted for semiannually for 22 wells in and around WAG 6. 173 RCRA-quality groundwater wells have been installed on the perimeters of the ORNL WAGs. Beginning in 1991 they are being sampled semiannually for metals, radionuclides, organics, and other parameters as part of a Remedial Action Monitoring Program. 
Table 21 (cont.)

2. The ORNL ER program supports several investigations of groundwater quality and movement as part of the ERMA (Clapp et al. 1991). The Source Term Definition project is investigating the diffusion of contaminants into soil and rock matrices and the creation of secondary source areas for contaminants. The stormflow zone project is investigating the role of hydrologic transport in shallow soil zones as a pathway of contaminant transport. The Hydraulic Head Measuring Stations (HHMS) are series of well clusters and single well that provide a means for defining the bounds of the uppermost aquifer and identifying pathways and capacities for contaminant transport.

3. DOE OHER supports studies of subsurface transport in a subsurface weir facility in the Melton Valley (Jardine et al. 1990) and studies of transport of contaminants from shallow soil zones (stormflow zone) and groundwater to small streams in the WOC watershed (Solomon et al. 1991).

Biota:

In support of NPDES permits and ER, the ORNL Biological Monitoring and Abatement Program (BMAP) monitors ambient instream toxicity at 15 sites in five streams near ORNL, studies bioaccumulation of contaminants in aquatic organisms, develops biological indicators for assessing exposure and ecological damage, conducts assessments of contaminants in terrestrial organisms, and is studying the radioecology of WOC and White Oak Lake. (See Loar et al., 1991)

\section{Other data:}

1. RI/FS for the ORNL WAGs provide groundwater data for WAGs undergoing remedial efforts. RIs and RFIs collect short-term information for surface water discharges and contaminant fluxes. These projects provide data for contaminants in soils and biota, they identify contaminants of concern via risk analysis, and estimate contaminant fluxes out of the WAG (i.e., into WAG 2).

2. The Clinch River RI (CRRI) provides data for contaminants released from the WOC watershed based on concentrations in water and accumulation in sediments and biota in downgradient areas. 
the BMAP is the long-term monitoring of contaminant levels in aquatic and terrestrial species and the identification of ecological indicator species that will serve to identify changes in biologically available contaminant levels over time as the ER programs progress. Key BMAP personnel have been incorporated into the WAG S\&A program.

Incorporating personnel from other activities into the WAG 2 RI provides needed expertise and helps to facilitate cooperation among these activities. Cooperating and collaborating on data acquisition and assessment benefit all activities and allow more efficient use of the available resources.

\subsection{Organization of the Staff for the S\&A Activities}

The organizational structure of the WAG 2 RI project staff has been modified from that presented in RI Work Plan to better serve the needs of the project (Fig. 2.4). As our understanding of the system has developed and as our data needs have become clearer, staff have been added to provide support in critical areas. A listing of the current staff is presented in Appendix A. The WAG 2 RI S\&A organizationa! chart (Fig. 2.5) depicts the functional relationships among the components of the WAG 2 RI project and other projects at ORNI.

Site characterization and monitoring group. The surface water hydrology group provides support for the measurements of surface water discharge needed by other components of the WAG 2 project to develop mass balances and contaminant flux estimates, and to mod it contaminant transport. The surface water hydrology group is linked to the ERMA program (see Sect. 2.3.1), which provides summaries of hydrologic data collected in the WOC watershed.

A surface water chemistry group has been developed to evaluate data generated by other projects conducting surface water monitoring programs in compliance with ORNL's NPDES permit and DOE orders. Those data have generally been useful for identifying contaminants of concern and contaminant sources. As additional information is gathered on groundwater chemistry and chemical signatures of seeps and stormflow zone discharges, surface water monitoring data may become especially useful for identifying and quantifying hydrologic and contaminant inputs.

A seep and stormflow group has been developed. Seeps are groundwater discharges that are important links between groundwater and surface water. Seeps will be important locations for quantifying contaminant inputs into surface water and can provide information useful for evaluating sources and fluxes of contaminants via subsurface flows. Stormflow is a pathway for hydrologic and contaminant transport that links subsurface and surface water. These components have been linked because of similarities in their approaches to monitoring. This group will develop technioues to locate, characterize, and monitor contaminant fluxes entering surface water via seeps and contaminants moving via stormflow. This group will coordinate with investigations of stormflow and secondary source areas supported by ERMA and DOE Office of Health and Environmental Research. This group is necessarily linked to the groundwater and surface water groups.

The groundwater group reptesents the diversity of geological, hydrogeological, and geochemical expertise needed to address groundwater issues and is drawn from several programs at ORNL as well as external sources. This group will primarily function to collect 


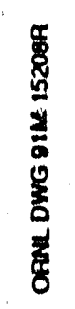
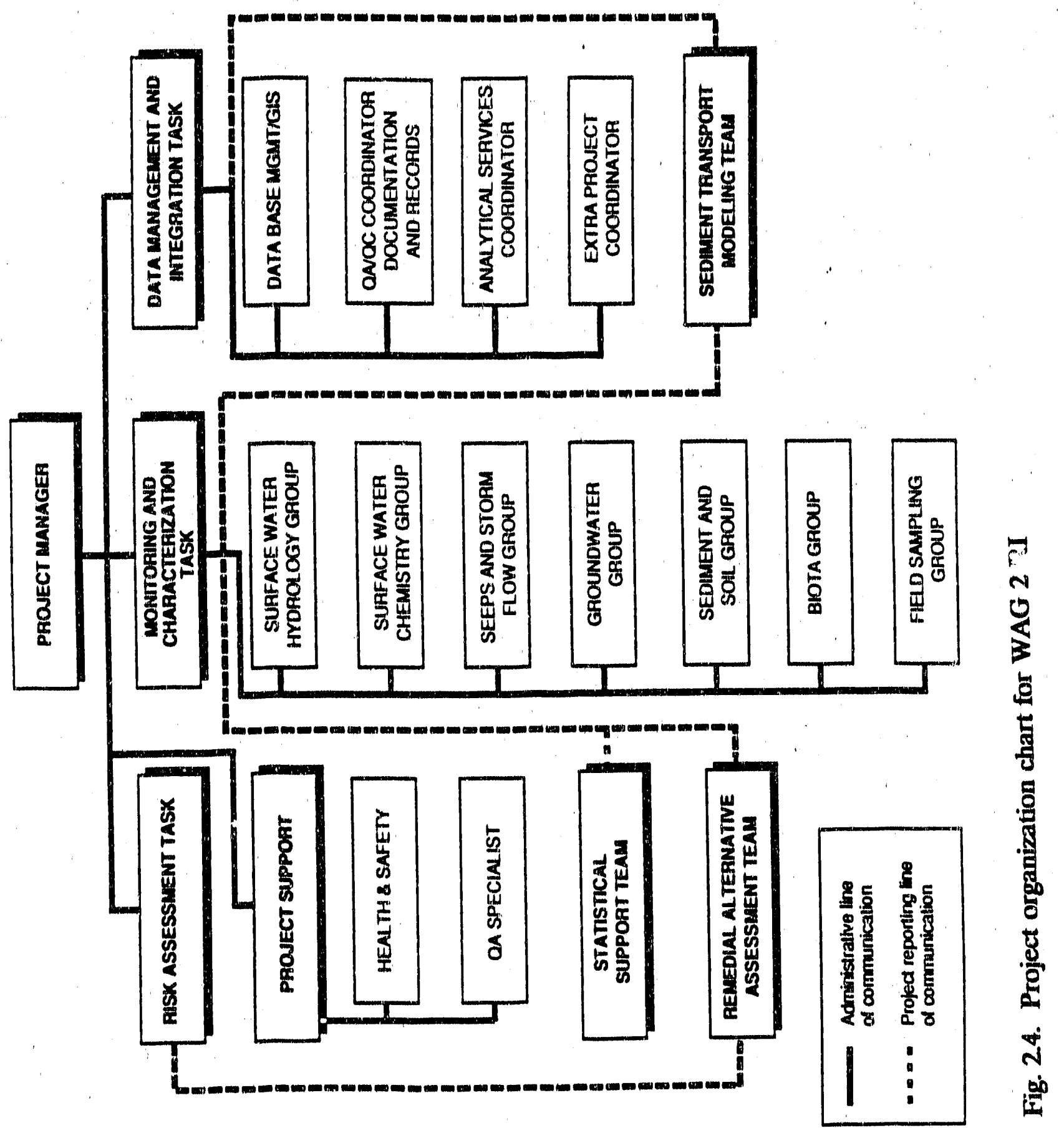


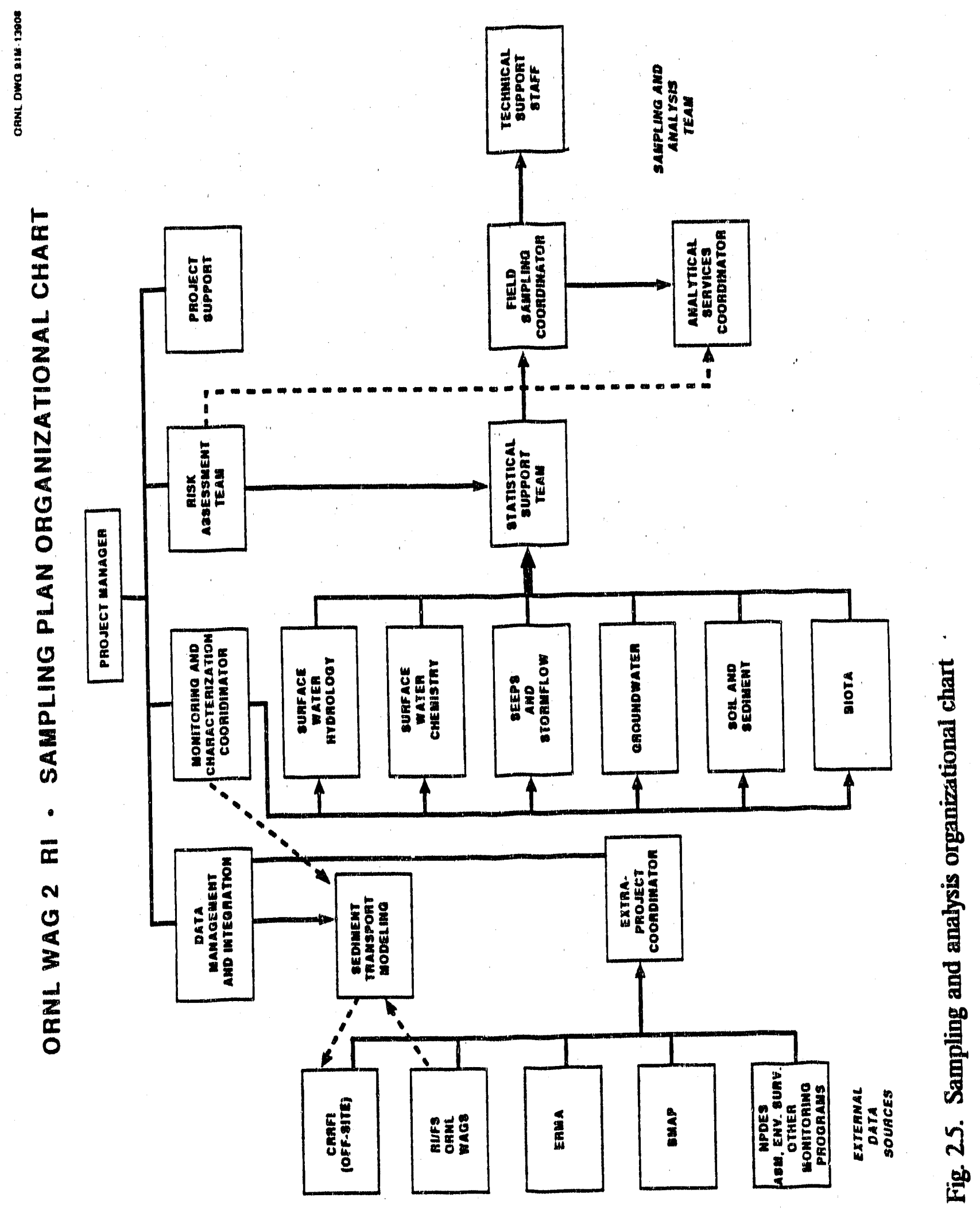


data and information generated by other efforts (e.g., ERMA and environmental surveillance groundwater data) for the hundreds of groundwater wells in WAG 2. This group will develop a data base for evaluating groundwater questions such as the distribution and sources of contaminants to the groundwater, processes determining the transport of contaminated groundwater from source WAGs into WAG 2 surface waters, and related issues.

The floodplain soil and sediment group focuses on the inventories and distributions of contaminants in soils and sediments in the WAG 2 system. This group takes a watershedlewel perspective to address questions such as the effects of the input of uncontaminated sediments from upgradient areas on contaminant tluxes in WAG 2.

The biota group will largely serve as an interface with the BMAP and ecological assessments for RI/FS projects for the ORNL WAGs. The activities of this group will emphasize the role of Wi 32 as an integrator of contaminants and a focal point for coordination and identification of data needs for the watershed.

Statistical support team. A statistical support team will provide support for sampling design, estimates of uncertainty, data evaluation, modeling, and related efforts (see Sect. 2.3.3). The group plays a critical role as the interface between the site characterization and monitoring teams, the risk assessment team, and the field sampling coordinator to ensure that sampling design and data collection allow accurate and unbiased estimates of risk with accurate estimates of uncertainty.

Sediment transport modeling team. As noted, sediments are the major pool for contaminants in WAG 2, and the transport of contaminated sediments during high-flow events is the primary pathway for contaminant movement off-site. The sediment transport modeling team will interface with modeling efforts for the ORNL. WAGs with active RI/FS projects and with the Clinch River RI to provide nested models to predict contaminant transport out of the WOC watershed under future conditions (e.g., extreme flow following watershed modifications). These data will allow the estimation of risk to off-site areas from individual ORNL WAGs and so provide information to help guide remedial actions for the ORNL WAGs.

Extra-project coordinator. As WAG 2 integrates contaminant releases from the watershed, the WAG 2 RI S\&A plan can serve as a focal point to integrate a diverse array of sampling and monitoring efforts underway in the watershed. A listing of external data sources for the WAG 2 RI has been presented in Sect. 2.3.1. The WAG 2 RI project takes advantage of these sources of information to provide a foundation for addressing issues important for environmental restoration of the ORNL WAGs and for addressing environmental issues related to contaminants in the WOC watershed. This integrating and assessment role beyond the boundaries of WAGs has been integrated into the activities of the ERMA project. The extra-project coordination role has evolved into a group effort to establish linkages, exchange information, and generally cooperate with other activities in and adjacent to the WOC watershed.

Remedial alternatives assessment team. A team representing engineering disciplines will evaluate potential remedial alternatives and help guide sampling and analysis. This team will ensure that the data gathered will be useful for evaluating potential remedial alternatives and identifying additional alternatives as new information becomes available. The team will include the site characterization coordinator. 
Other components and responsibilities remain unchanged from those presented in the RI plan (ORNL 1990).

\section{STATISTICAL SUPPORT}

As mentioned previously herein, the first 2 years of effort are primarily a scoping study. These data obtained from these investigations will be used to determine issues relating to sampling. For example, these data will be used to determine the sample size necessary to estimate a given contaminant to within some margin of error with a given probability. The data will help shed light on the variability of contaminants in the field. It will also belp to determine possible sampling strategies. For example, the WAG 2 area may be broken down into strata on the basis of the scoping study and a stratified sampling plan could then be used in future work.

Scoping studies are themselves initial investigations, and statistical issues such as uncertainties, power of statistical tests, and sample size determinations are generally not a part of the scoping study because the information to calculate these are unavailable. In fact, the scoping studies are intended to provide the information that is needed to estimate these parameters. The statistical support during this phase of the study will be in the form of collecting and analyzing the data collected during the scoping study to answer these questions needed to design a more formal and thorough investigation.

The statistical needs of the study are varied and cover a much broader spectrum of statistical techniques than the area of sampling alone. The areas of statistics that will be involved in the scoping study include:

1. Estimate parameters and confidence intervals.

2. Develop empirical models relating contaminant flow to other variables.

3. Perform sensitivity/uncertainty analysis of computer models, which can be used to determine where sampling efforts should be made.

4. Develop techniques to handle nondetects observed in the analysis of samples.

5. Design sampling plans based on prior information and data from previous studies.

6. Identify trends in the data.

7. Predict extreme events, for example, 100-year rainfalls.

8. Develop sampling schemes to identify "hot spots."

9. Use data collected in the gamma scan walkover to determine relationships with contaminant deposition and develop sampling plans.

10. Use time series techniques to develop predictive models.

11. Help to develop a viable number for background levels of contaminants.

12. Apply the appropriate statistical tests for testing hypothesis and determining the power of such tests.

13. Modeling the contaminant mass flux into, through, and out of WAG 2.

14. Calibrating automatic samplers.

15. Determine when compositing is a more effective method of sampling than simple subsample analysis.

16. Develop methods to interpolate/extrapolate sampled data to develop contour plots.

17. Develop cost/benefit analysis to accompany sampling plans.

18. Develop techniques to help visualize the data obtained and explore its multivariate structure. 
19. Employ techniques, such as cluster analysis to determine areas that are similar in their measured response.

20. Help in the quality control efforts of the study.

The above is a partial list of the areas of statistics that are involved in the scoping study. It is obvious that the needs are quite varied and the relative effort for these tasks will be also.

\section{QA PROJECT PLAN FOR SAMPLING AND ANALYSIS}

The W 2 Quality Assurance Plan (in review) contains the full QAVQ Program Plan, which addresses all the elements of the complete QA package. Identification of WAG 2 personnel roles and responsibilities are found in the WAG 2 RI Plan (ORNL 1990). This section will address those special quality issues that are specific to the sampling and analysis processes discussed in Sects. 3 through 6 of this document. WAG 2 is unique in purpose and plan; however, the project will provide data of acceptable quality. ERD requirements for quaiity control of analytical data (Energy Systems 1990) serve as a basis for development of this plan.

The objective of the QA/QC Plan is to develop and ensure implementation of procedures for field sampling, laboratory analyses, chain-of-custody, data reporting, and information handling that will provide data of known quality that is legally defensible. This plan uses existing data, data still to be collected by other programs, and data to be obtained by this S\&A plan. Data that do not originate or are not collected under this plan are subject to the quality assurance requirements of the program under which the data were collected. Data from measuring and monitoring activities collected by WAG 2 staff under this S\&A plan are subject to the quality assurance requirements defined in Sect. 12 of the WAG 2 RI Plan (ORNL 1990). Procedures described in this S\&A plan are intended to ensure that the QA/QC goals established for the WAG 2 RI Plan are achieved. They are also intended to minimize field measurement errors, analytical errors, and errors occurring in data transfer and management. Project-specific procedures are located in the WAG 2 RI Standard Operating Procedure Manual and are controlled by the WAG 2 Quality Assurance Coordinator. The mechanisms employed to ensure quality are

- prevention of defects in quality through planning and design, documented instructions and procedures, and careful selection of personnel;

- assessment through regular audits and surveillances to supplement continual informal reviews; and

- permanent correction of conditions adverse to quality.

This QA/QC plan is designed to comply with QAMS-005/80 and ANSI/ASME NQA-1 guidelines. EPA QAMS-005/80 (EPA 1980a) contains EPA's guidance for project QA/QC plans. ANSI/ASME NQA-1 (ANSI/ASME 1986) has been adopted as the main QAVQC standard. This project falls under both RCRA and CERCLA regulation; therefore, this project QA plan is subject to OSWER Directive 9502.00 6C (EPA 1987a), OSWER directive 9355.0-76 (EPA 1987b), and OSWER Directive 935:3-01 (EPA 1988b). This plan is structured around the QAMS-005/80 guidelines (EPA 1 980a) but is cross-referenced in the NQA-1 requirements of the WAG 2 plan. Because of the QA programs imposed by regulatory agencies, this project has a category I QA rating (Roberson and Logsdon 1989). The NQA-1 Modular Profile shows the relationship between the elements of this plan, those of the ORNL QA Program, and NQA-1 (Table 2.2). 


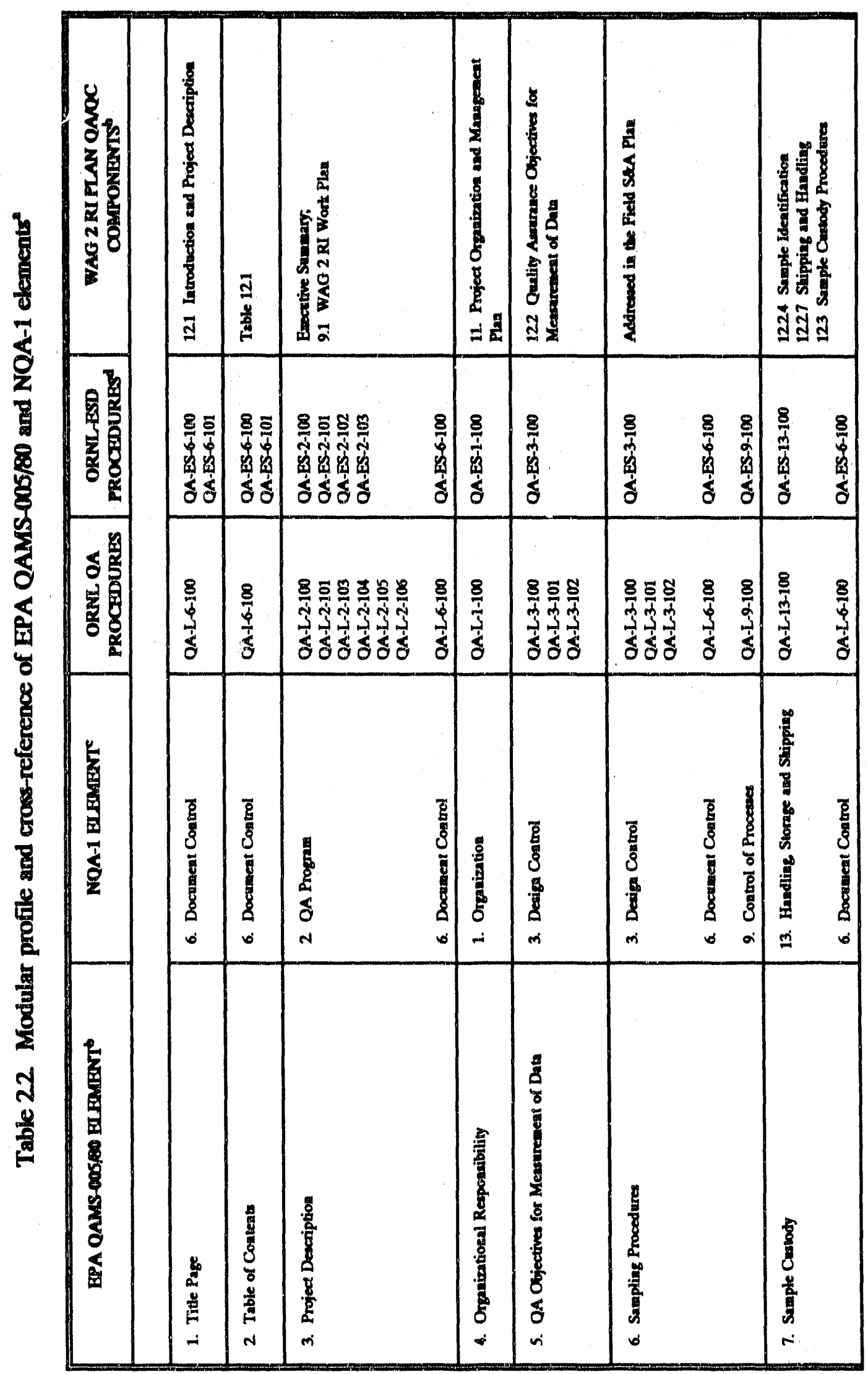




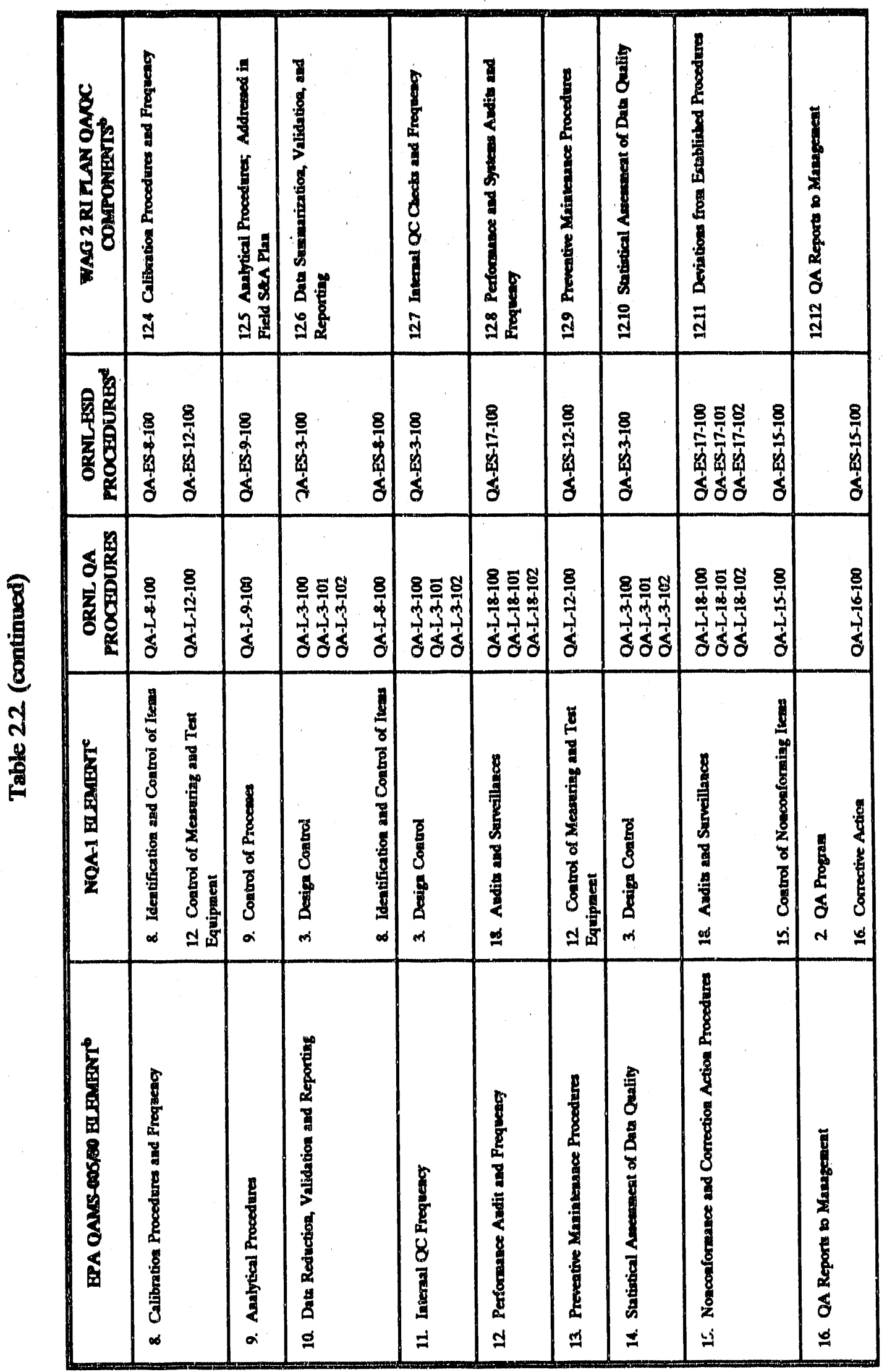




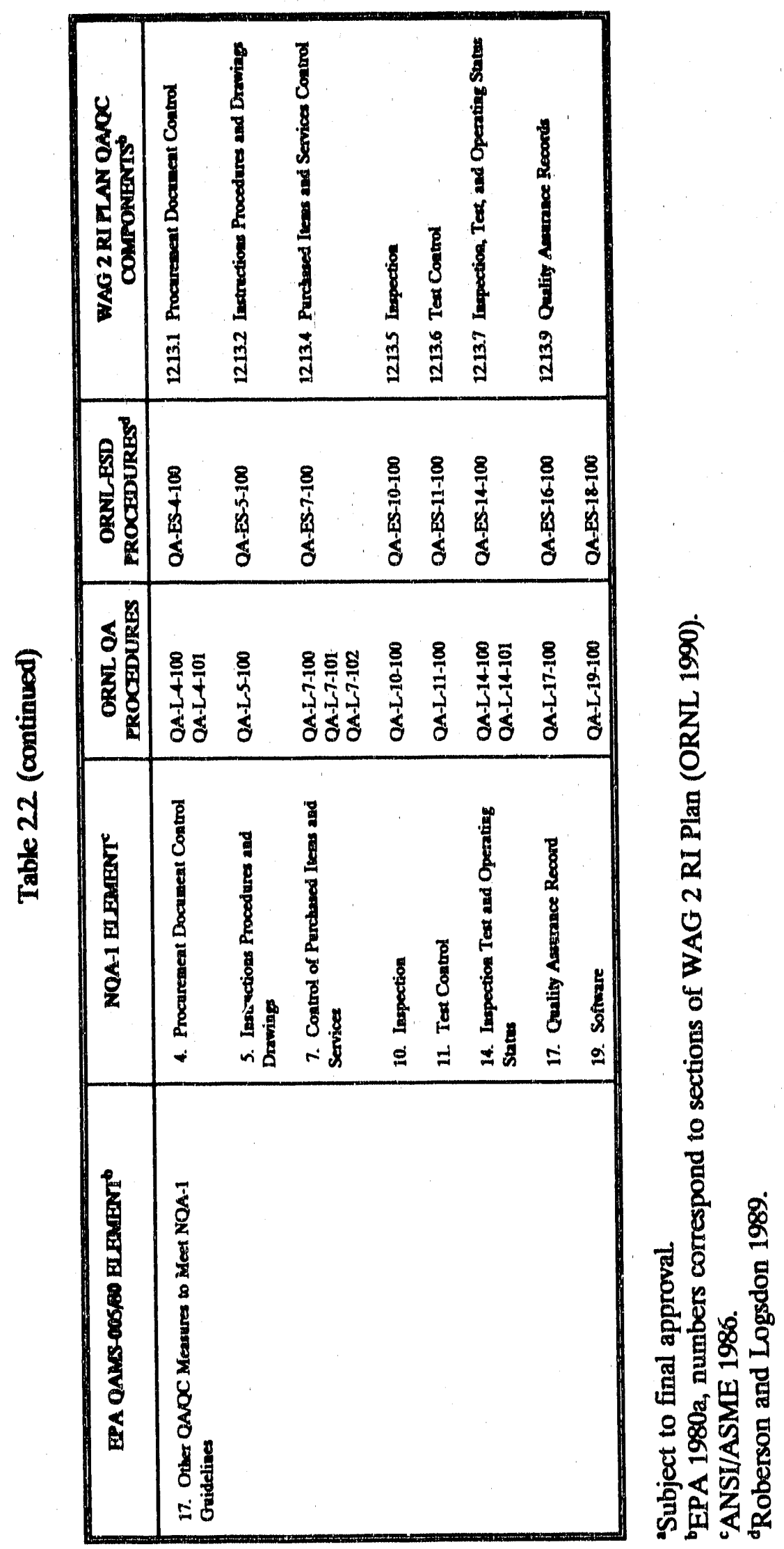




\subsection{Project Requirements for QA Awareness and Training}

All organizations performing tasks for the WAG 2 RI must have a QA plan that meets WAG 2 data quality objectives (Sect. 2.5.4). Review of the QA plans of other organizations is the responsibility of the extra-project coordinator, the WAG 2 Quality Assurance Coordinator, and the project manager. If there is no approved QA plan for the task, then the organization will be required to develop an acceptable plan. It may be necessary to add project-specific attachments such as organization charts, functional responsibility matrixes, and surveillance plans to otherwise adequate plans. Interface agreements will be developed with all organizations performing tasks for WAG 2. These interface agreements will provide configuration and change control statements between organizations.

All ORNL and subcontractor personnel working on the WAG 2 RI will be properly trained, qualified individuals. QA awareness will be addressed by information sessions and distribution of the project QA plan. The QA coordinator w ll be responsible for conducting the sessions and distributing the plans. Receipt of information shall be documented by attendance and document sign-out sheets. Prior to commencement of work, all sampling team personnel will be given instructions specific to the remedial investigation covering the following areas:

- training needs assessment

- organization and lines of communication and authority

- description of the WAG 2 system

- overview of the S\&A, QA/QC, H\&S, and Data Management Plans

- documentation requirements

- personnel protection procedures

- waste management procedures

- decontamination procedures

- emergency procedures

\subsection{QA for Data from Other Projects}

Data from several other projects will be utilized in the WAG 2 RI (Fig. 2.2). The ExtraProject Coordinator will be the point of contact for interactions with these various groups (i.e. CRRI, ORNL WAGs RI/FS, ERMA, BMAP, NPDES, ASEMP, and ESP). Data from these groups will be transferred to the Data Management and Integration Group. The QA plans developed for these groups will be reviewed to ensure the data meet WAG 2 data quality objectives. WAG 2 QA staff will review the data and data quality objectives to ensure that the data are appropriate for the proposed application in WAG 2 . WAG 2 staff will also review or participate in audits and surveillances to ensure that the data are being collected and handled in the manner prescribed in the QA plan of the group providing the data.

\subsection{QA Objectives for Measurement of Data}

This section implements applicable regulatory requirements and provides internal control and review so that the data are scientifically sound and legally defensible. 
QA objectives for data are that

- scientific data generated will withstand scientific scrutiny;

- data will be gathered using appropriate procedures for field sampling, chain-of-custody, laboratory analyses, and data reporting; and

- data will be of known precision and accuracy.

The QA requirements for the WAG 2 RI are defined in Sect. 12 of the WAG 2 RI Plan (ORNL 1990). The procedures to be used for assessing the quality of field and analytical laboratory data are described in Sect. 2.5.6. Analytical laboratories selected for this project must meet criteria for laboratory certification and adherence to regulator $Q A$ requirements.

\subsection{Data Quality Objective}

Data quality objectives (DQOs) are derived from the intended uses of the data. To develop DQOs, environmental variability and analytical quantitation requirements must be considered along with measures to evaluate the precision, accuracy, representativeness, comparability, and completeness of both sample collection and analysis, and the level of documentation required to support the intended use of the data. Because this S\&A Plan emphasizes scoping and screening activities, the sampling locations, number of samples, and methods of data collection are intended to provide information for locations, media, and/or analytes for which few data exist and to estimate environmental variability (Sect. 2.2 of this document). Therefore, consideration of environmental variability has not been included in the development of DQOs for this stage of the S\&A activities. The analytical methods selected are based on quantitation limits (levels of concern) required to identify contaminants of potential concern (e.g., evaluate ARARs, conduct risk-based contaminant screening, establish background conditions, etc.). Data meeting historic method quality control criteria therefore meet the analytical requirement of the DQO for this project.

To meet project data use requirements, measurement tasks will be assigned the appropriate DQO (QC documentation) level (I-V) as defined in Data Quality Objectives for Remedial Response Activities (EPA 1987c). Data of two different quality levels will be generated during field investigations. Field data such as radiation monitoring will meet the requirements of Levels I or II data quality. Analytical laboratory data collected for surface water, groundwater, sediment, and biota sampling will meet the requirements of Level III or Level IV data quality. For example, Level III data quality are obtained from field screening exercises using portable instruments. Results may not be quantitative or compound specific but are inexpensive and can be made available quickly. Level I and II data can be used for scoping and screening such as (1) initial delineation of contaminated zones, (2) crude presence or absence of contaminantion, and (3) gross determination of analytes in samples. Level III and Level IV data quality requirements provide laboratory analyses using standard EPA methods such as those in SW-846, EPA 600, and the Contract Laboratory Program (CLP). Level III provides information for a wide array of analytes with known accuracy and precision. Level IV data in addition provides rigorous QA/QC protocols and documentation and provides qualitative and quantitative analytical data which is legally defens ible. Level III and IV data are suitable for site characterizations and risk assessments. The data quality criteria for Level III and IV are (1) detection limits for each procedure will be consistent with EPA-approved methods; (2) laboratory QAVC procedures will follow method specific requirements; and (3) field QAVC procedures will be assessed by reviewing data generated from the analysis of trip blanks, equipment rinsates, field blanks, and duplicate samples. 
The determination of the appropriate QA level for data collection (field measurements or analytical methods) will depend on the intended use of the data. If the data are to be used exclusively for screening (i.e., to determine the relative concentration of a contaminant, its concentration, or evaluate its presence or absence), Level I or II QA will apply. This includes data used to extrapolate to average concentrations or fluxes of contaminarits. For example, the gamma activity walkover of the floodplain will generate thousands of data points. Based on the data from the walkover, soil samples will be collected and analyzed for gamma-emitting radionuclides, and these data will be compared with the data resulting from the walkover to establish a relationship between the field measurements and laboratory analyses. Data from the walkover can then be used as a basis for extrapolation to average levels of gamma activity in the floodplain for preliminary risk analysis. In this example, the field screening would be QA Level II and the laboratory analysis would require QA Level III.

Because contaminant inputs into WAG 2 are changing (as upgradient WAGs are remediated), the nature and extent of contamination is also changing. Therefore, a baseline risk assessment will follow the remediation of upgradient WAGs in 10 to 20 years. Data collected in the interim period by the multimedia environmental monitoring program will be used to track contaminant inputs, releases, and inventories. Data collected during the interim period will form the basis for an abbreviated and very specific sampling and analysis program for the baseline risk assessment. Thus, the majority of data collected by the multimedia environmental monitoring program will require no more than Level III QA. However, because the WAG 2 RI Project involves a long-term monitoring effort and analytical methods that will likely change over the duration of the monitoring effort, approximately $10-25 \%$ of the data collected will meet the requirements of Level IV QA. The Level IV full CLP-like data packages will provide comparison of current and future analytical methods and will ensure the ongoing usability of data for the duration of the WAG 2 RI Project.

DQOs may include analytical Level V (special methods) for some contaminants. For example, the risk-based contaminant screening found potentially significant risks associated with certain organic contaminants that were not detected but which were screened at a concentration equal to the detection limits (Blaylock et al. 1991b). For these organic compounds, either analytical methods with lower limits of detection will be used, or compounds will be eliminated from further consideration based on evidence such as no reported detection in any media and no known sources in the watershed. A rationale for dealing with contaminants for which Level III or IV analytical methods may not provide acceptable detection limits is being developed in conjunction with Energy Systems' Central Risk Assessment Committee and will be presented in the next iteration of the S\&A Plan prior to the next and more intensive round of field sampling.

The WAG 2 RI risk assessment team, in consultation with the project manager, the site characterization coordinator, and the ER-APO, will determine the QA level required for all measurements and activities. In general, if data are to be used directly for risk assessment, feasibility studies, or remedial design, QA Level III or higher will be required.

\subsection{Quality Control Data}

Quality control samples will be used to screen out data of unacceptable precision or accuracy and thus provide data defensibility. Depending on the time frame in which the data are requested, a variable in the data set would indicate the current level of data quality and the target level. Section 2.5.14 discusses data reduction QA procedures in more detail. This 
project will follow the definitions for precision, accuracy, completeness, comparability, and sensitivity given in the Handbook for Analytical Quality Control in Water and Wastewater Laboratories (EPA 1979a).

\subsection{Field Activities}

The multimedia environmental monitoring plan emphasizes the dynamic aspects (movement into and out of) the system. The equipment and the techniques that will be employed to obtain representative samples will bo in accordance with approved Environmental Surveillance Procedures (ESP-300 series, Kimbrough et al. 1990). However, established methods are not available for certain procedures (e.g., locating groundwater discharge areas, or calibrating point withdrawals by automatic samplers with actual whole channel fluxes). Therefore, we will be developing and evaluating several project-specific procedures. Where possible, existing procedures will be modified for these purposes. Where no similar procedure exists, a draft or guiding procedure will be developed, approved, and used to facilitate the documentation process. All project-specific procedures developed will be reviewed by the ER-APO. All modifications in methods will be documented and made part of the ERD DMC.

Records documenting the modifications evaluation, verification, and other aspects of procedure development will follow documentation procedures described in the WAG 2 RI QA plan. A copy of all WAG 2 field and laboratory procedures will be maintained in a controlled Standard Operating Procedure (SOP) Manual according to guidelines described in the WAG 2 RI plan. The SOP Manual will be readily available to WAG 2 personnel conducting laboratory or field activities. Draft copies of procedures under development and references for procedures to be developed are located in Appendix C.

The QA completeness objective for this project is to obtain valid, analytical results for at least $95 \%$ of the samples collected during the project. Accountability of samples collected, from field to final disposal, must be $100 \%$.

Objectives for comparability between samples are met by the following: (1) narrowly defined sampling methodologies; (2) site surveillance and use of standard sampling devices and monitoring devices; (3) training of personnel; (4) documentation of sampling point; (5) stringent control limits for the QC checks; and (6) reporting results in appropriate, comparable units.

Accuracy among monitoring instruments is determined by comparison of readings during calibration to a standard. Precision in sampling is measured through the use of field duplicates. Accuracy is addressed by the use of standard criteria for container and equipment cleaning, standard sample collection protocol to obtain a representative sample, personnel training and performance criteria, uniform sample-handling techniques, and blanks to detect contamination. Representativeness of samples collected is controlled through adherence to the sampling plan and to detailed descriptions of sampling procedures described in Sects. 2 through 6 and corresponding standard operating procedures.

Measures to attain sensitivity objectives include (1) uniform training and certification for staff; (2) standard provisions for inspection, maintenance, and repair; (3) provision of standard operating procedures (SOPs) to technical staff; (4) reference of SOPs in the S\&A Plan; and (5) field inspections by the QAVC coordinator to determine compliance with the items 
specified in the support plans. All aforementioned measures to attain sensitivity objectives are described in the WAG 2 QA Plan (in review).

Staff responsible for particular instruments must maintain a log of calibration procedures and results that will remain with the instrument as a means of establishing a record of calibration. The sampling team leaders will inspect and initial such logs, which will become part of the project records. Each operator must be trained in the proper use of the instrument, be familiar with the instrument's use, and be able to properly interpret data from the instrument.

\subsubsection{Freld documentation}

An integral part of the QAVQC Plan for the field activities will be to maintain accurate and complete field recotds, including logbooks and appropriate field data forms. Field logbooks shall be of hardcover construction with stitched binding and water-resistant pages. Information identified in these records will be obtained from the site exploration and sampling activities and will be reviewed by the sampling team leader. All information pertinent to field activities will be recorded. Entries in the logbooks or on the data forms will be made in water-resistant ink and will include at a minimum:

- date and time on-site;

- the names and affiliations of field personnel;

- a general description of the day's field activities;

- documentation of current weather conditions as well as weather conditions during the previous 48 hrs when appropriate;

- field equipment and instrument number;

- field equipment calibration data, when required;

- field readings from personnel safety instruments; and

- field data measurements such as temperature, conductivity and pH.

Appropriate forms for field generated data will be prepared based on the requirements in the WAG 2 RI Plan (Sect. 12, ORNL 1990).

\subsubsection{Field data management}

Field records will be recorded in permanent ink, legible, and sufficiently complete to permit reconstruction of data-gathering activities by a qualified individual (other than the originator) when data are reduced. Field notebook entries should be consistent, factual, detailed, and objective. The field records will be the basis for later written reports, and all entries must be free of terminology that might prove inappropriate. The method of data reduction will be identified and recorded. Field-generated data sheets will be collected and reviewed daily for accuracy and completeness by the field sampling coordinator before being transferred to the Records Control Manager. Standard Operating Procedures (SOPs), Use of the Document Management Center, Forms Development and Control, and Forms Acquisition and Post-Sampling Process further describe project-specific field data management procedures. All SOPs are located in the controlled WAG 2 SOP Manual distributed by the Quality Assurance Coordinator. Manual entry of field data will be coordinated by the Data Base Management Group. Data entry will enter field data into specified computer systems to facilitate retrieval by WAG 2 RI personnel. Quality will be checked by double entry and verification of entered data. The WAG 2 RI project manager will forward field notebooks to the DMC at the conclusion of field activities. 
The Sample Information Management System (SIMS) protocols (BNI 1989) for sample identification and tracking using bar coding technology will be used in the field sampling and analysis. The procedures defined in this document applies to all ORNL RI/FS project field sampling projects. Sample labels or tags will contain sufficient information to identify the sample in the absence of other documentation. The label or tag will be directly affixed to the sample container and any handwritten information will be completed using indelible ink. Labels will include at a minimum:

- project name,

- unique samplo number,

- sample location,

- sampling date and time,

- signature of individual collecting the sample,

- preservation method employed, and

- comments regarding sample characteristics.

See the WAG 2 RI SOP Manual for the procedure on sample identification (in preparation).

\subsubsection{Field custody procedurea}

Field custody procedural activities include the following steps.

- Before sampling begins, the WAG 2 RI QAVQC coordinator will instruct sampling personnel on the chain-of-custody and sample labeling procedures, as necessary.

- A chain of-custody record will be initiated in the field for each sample, corresponding to the sample identification label.

- Sample preservation steps will be recorded on the field chain-of-custody record.

- Each time sample custody is transferred, the person relinquishing the sample and the new custodian will sign the record, note the date, and indicate the reason for transfer of custody.

- The analyses to be performed for each sample will be recorded on a request-foranalysis form.

- The sampling team leader and field services coordinator will confirm that proper custody procedures and report forms were used during the ficldwork and that results were documented in the field logbook.

- Samples transferred to analytical laboratories will be recorded in the field logbook by the ASC at the end of the collection period.

See the WAG 2 RI SOP Manual for the procedure on chain-of-custody (in preparation).

\subsubsection{Field QC Sampling}

Field QC sampling will be established to check sampling and will constitute $5-10 \%$ of the total number of samples. All QC samples will be shipped according to the chain-of-custody procedures specified in Sect. 7 of this report. Field QC samples will include blanks and replicates as follows:

Field Rinsate. A field rinsate consisting of final rinse water from the decontamination of field sampling equipment. Analysis of the field rinsate determines if the decontamination procedure is adequate to avoid carryover of contamination from one sampling location to another. A field rinsate will be collected at a minimum of 1 in 20 cleanings of any given piece of equipment. 
Laboratory rinate. A laboratory rinsate consists of final rinse water from the decontamination of field sampling equipment. Analysis of the laboratory rinsate determines if decontamination procedures are adequate. Laboratory rinsates will be collected prior to each day of field activities or at a minimum of 1 in 20 cleanings of any given piece of equipment.

Field blank. One field blank consisting of source water (distilled/delonized water) used for decontamination will be collected for every 20 samples or once per sampling event, whichever is greater. Field blanks will also be used to detect airborne metal or organic contaminants present at the time of sample collection. One field blank container consisting of distilled/deionized water will be opened during the collection of one in twenty metal or organic samples.

Field duplicate. Field duplicates, which consist of a duplicate sample from one sampling location, indicate whether the field sampling technique is reproducible. Duplicate samples will be obtained at a collection frequency of 5 to $10 \%$ for all sample matrices.

Field QC samples will have discrete sample numbers and be submitted as "blind" to the laboratories. The quantities and collection procedures for each field QC sample type are specified in Sects. 3 through 6 for each environmental media and in SOPs, as appropriate. Results of these samples will be included in the analytical data report. Results for QC samples will not be used to adjust the results obtained for original samples. If contaminants are found in the blanks, attempts will be made to identify the source of contamination, and corrective action will be initiated according to Sect. 12.13 of he WAG 2 RI Plan (ORNL $1990)$.

\subsubsection{Field analyses}

All field measurements will be recorded in the field notebooks or on specially designed data forms. All data will be directly entered in the field, signed, and dated. If entry changes are made, one line will be drawn through the error, and the change and explanation will be signed and dated in the notebook or on the data form. Changes made to original notes should not obliterate the original information. All field data records will be organized into a standard format, when possible. The field sampling coordinator is responsible for periodic review of daily entries in the field logbooks.

\subsubsection{Laboratory Activities}

Laboratory statements of work will be developed in conjunction with the ER-APO, will specify $\mathrm{QC}$ requirements and $\mathrm{QC}$ sample deliverables, and will be submitted to the laboratory for approval prior to sample shipment. The laboratory will be required to submit the results of requested control sample analyses and other QAVQC documentation (Sect. 2.5.13) to the WAG 2 RI ASC to ensure conformance with established control limits and other QA requirements.

The QA completeness objective for this project is to obtain valid analytical results for at least $95 \%$ of the samples collected. Laboratory completeness will be deternined by the extent to which data are substantiated by hard-copy documentation, which includes chain-ofcustody, requests for services, and instrument calibration forms. Comparability and sensitivity 
criteria are established by either the most recent statement of work or SOPs for particular analytical laboratories.

\subsection{Prevention of Crowa-Contamination}

Sampling equipment will be decontaminated before use and between each sample per ESP-900 (Kimbrough et al. 1990). Each decontamination activity will be recorded in the field logbook or a decontamination notebook. Specific procedures for equipment decontamination are included in the SOP Manual for the sampling procedure in which the equipment is used.

\subsection{Sample Turnaround Time}

Sample analyses will be scheduled according to site investigation needs consistent with the sample holding times as defined in Requirements for Quality Control of Analytical Data (Oak Ridge K-25 Site, 1991). The S\&A Plan is organized to provide a turnaround time that will meet the project schedule and objectives. Specific sample holding times are found in Table 7.1 of Requirement for Quality Control of Analytical Data (Oak Ridge K-25 Site, 1991). These requirements shall be included in any contractual agreement between the WAG 2 RI Project and contract laboratories.

\subsection{Shipping and Handling}

Handling, shipping, and storage of samples and data resulting from field activities will adhere to custody and safety procedures described in the WAG 2 RI Plan (ORNL 1990, Sects. 12.3 and 14). Specific shipping and handling procedures for each sample medium are being developed in the QA Plan for each media being collected.

\subsection{Variance System}

Procedures that properly anticipate all conditions encountered during a field sampling program cannot be prepared. Variances from approved operating procedures in the WAG 2 RI Plan, the S\&A Plan, the QAVQC Plan, or the H\&S Plan will be documented in a deviation request form, described in Sect. 12 of the WAG 2 RI Plan (ORNL 1990).

The field sampling cuordinator will initiate and chronologically maintain a variance log. A variance requires the approval of the WAG 2 RI project manager and the Quality Assurance Specialist (QAS) before work proceeds. As appropriate, regulatory agencies will be notified of any variances that significantly affect project scope or objectives. Any variances from the H\&S Plan must be approved by the H\&S Coordinator. Approval by the WAG 2. RI project manager and QAS can be initiated on a verbal basis via the telephone or radio with follow-up sign-off. In no case will non-WAG 2 RI project personnel initiate a variance. Copies of the deviation request forms will be maintained by sampling teams until the fieldwork is complete and will then be forwarded to the WAG 2 RI project manager and sent to the ERD/DMC in a procedural change notice according to ER/C-P1100, "Initiation, Review, Revision, Approval, and Issuance of Environmental Restoration Procedures and Instructions." 


\subsection{Sample Custody Procedures}

Chain-of-custody procedures require documented sample possession from the time of collection to disposal, in accordance with Energy Systems procedure ESP-500 (Kimbrough et al. 1990). These procedures are discussed in Sect. 7 of this report. Additional details of document control are included in Sect. 13 of the WAG 2 RI Plan (ORNL 1990).

\subsection{Analytical Procedures}

The procedures to be used in sample analysis are listed in Tables $3.3,4.3,4.4,5.2$, and described in text in Sect. 6 of this document. The samples will be analyzed for potential contaminants of concern using SW-846 procedures or other accepted or documented methods (e.g., EPA 1979b; 1980c; 1983; 1984b; 1986a,d; 1987d; Kimbrough et al. 1990, Appendices A.2 and A.4).

Laboratory QC samples will be used to check sample preparation and analysis and to monitor laboratory performance. ERD guidelines for laboratory QC samples and documentation have been established in Energy Systems (1990). Specific data deliverables vill be approved by the ERD-APO. Specific requirements for groups of samples will be specified in SOWs for those samples. Analysis-specific control samples may be required as indicated by IIPA accepted procedures. QC samples will consist of blanks, duplicates and spikes. Laboratory standards will also function as QC components. QA procedures for laboratory processing include laboratory duplicates of all field samples to determine the precision of laboratory results. Laboratory QC samples will include the following:

Method blank A method blank is a blank sample made up of a pure, noncontaminated substance of the matrix of interest (usually distilled/deionized water or silica sand) that is subjected to all of the sample preparation (e.g., digestion, distillation, and extraction) and analyticai methodology applied to the samples. The purpose of the method blank is to check for contamination from within the laboratory that might be introduced ding sample analysis.

Calibration/continuing calibration blank A calibration blank is the substance that is used to zero the instrument. The calibration blank comprises the solvent used for the preparation of the calibration standards and samples. The calibration blank accounts for any interferences from the solvent mairix.

Sample container cleaning blanks. If sample containers are cleaned in the laboratory, "sample container cleaning blanks" are taken for each batch of containers that goes through the cleaning process. If contamination is detected, the containers associated with the contaminated blank will be recleaned and another blank taken and analyzed.

Laboratory duplicates. Laboratory duplicates are prepared by the laboratory analyst for each sample and are obtained by homogenizing a sample as thoroughly as possible and taking two separate aliquots of that sample for analysis. The duplicate sample, however, should never be a method blank, trip blank, or field blank. The purpose of laboratory duplicates is to check the precision of the analyst, the sample preparation methodology, and the analytical methodology.

Matrix spikes. A matrix spike is an aliquot of a sample to which a known concentration

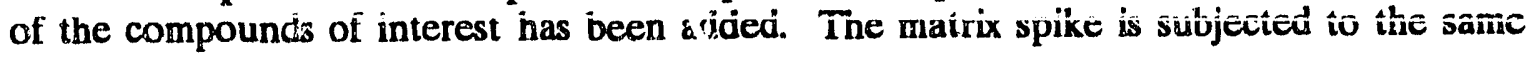


sample preparation and analytical methodology applied to the samples. The sample to be spiked is selected prior to sample submittal by the ASC; however, the spiked sample cannot be a method blank, trip blank, or field blank. The purpose of the matrix spike is to check for interferences or false readings caused by the sample matrix.

Blank spike/laboratory control sample (LCS). The blank spike, or LCS, is a blank sample (usually distilled/deionized water or silica sand) to which a known concentration of the compounds of interest has been added. The blank spike is subjected to the same sample preparation and analytical methodology applied to the samples. The purpase of the blank spike is to check the accuracy of the analyst, the sample preparation methodology, and the analytical methodology. The level of accuracy is measured by calculating the percent recovery $(\% \mathbf{R})$.

The laboratory QA/QC Coordinator is responsible for preparing QC standards and sending QC samples into the laboratory for analysis. Statistical analyses will then be performed utilizing the results of QC sample analyses. Each laboratory will apply precision and accuracy criteria to each parameter that is analyzed. When analysis of a sample set is completed, the QC data are reviewed and evaluated through the use of control charts to validate the data set. Laboratory QC standards will include the following:

Calibration standards. Calibration standards are standards comprising the compounds of interest at known concentrations. Calibration standards are prepared from EPA reference material or commercially available, certified reference materials traceable to the National Institute of Standards and Testing (NIST). Calibration standards are prepared using the same solvent used for sample preparation at the same concentration. Semivolatile and volatile organic analyses by GC/MS require one point calibration by current CLP criteria. Calibration standards for other methods require at least three concentration levels plus a blank standard throughout the calibration range required for the analysis. Calibration standards are not subjected to all of the preparation (e.g., extraction, distillation, and digestion) that is applied to the sample. Calibration standards are used to initially calibrate the instrument by providing reference points throughout the calibration range and to establish linearity throughout the calibration range and working range of the instrument. The instrument is then checked continually throughout the analysis with the calibration standards to check for any instrument drift that may have occurred.

Performance evaluation (PE) samples. Performance evaluation samples consist of known concentrations of the analytes submitted to the laboratory being audited. These samples are obtained through various EPA-sponsored programs and private vendors to provide an objective evaluation of laboratory performance and comparison with other participating laboratories.

Control charts are statistical representations of the laboratory's performance and are used to monitor laboratory performance and to establish control limits or the acceptance criteria for all compounds of interest. For each analyte, a separate control chart is required for each type of control sample that measures precision or accuracy (blank spike, matrix spike, and duplicate) and for each matrix type and concentration level (high, medium, and low). A minimum of ten measurements of precision and accuracy are required before control limits can be established. Control limits of three standard deviations shall be utilized for all samples. 
Once established, control limits are updated as additional precision and accuracy data become available to the laboratory $\mathrm{QC}$ coordinator. Any control sample data point that falls beyond the control lixnits or any data trend (indicated by seven or more consecutive points on either side of the mean) will require an investigation and corrective action. For all identified contaminants of concern, control limits and corrective actions will be in accordance with EPA protocol. Additional statistics for organics work will be done in accordance with SW-846 (EPA 1986c) or the CLP statement of work (EPA 1986d), as applicable.

\subsection{Data Sumraarization, Validation, and Reporting}

The data or validation process compares the objective versus the actual through the evaluation of the PARCC parameters. The laboratory will not perform data validation; laboratory QAVIOC data will be validated, independent of the laboratory by a contract organization. A statement of work describing data editing, screening, checking, auditing, verification, cartification, and review (EPA 1988b) will be implemented prior to the initiation of data validation. All data for this project will be evaluated by QA/QC methods and internal peer review. Data reduction, verification, and reporting will be in accordance with the ongoing ORNL ER Program Data Base Management activities (Voorhees et al. 1988, 1989; Hook et al. 1990). Data will be entered into common, standardized formats. In addition to following field sampling documentation and $\mathrm{QA} / \mathrm{QC}$ procedures, data are verified using a variety of computerized checks for reasonableness. These procedures will ensure that data are entered, encoded, and manipulated in a consistent way and available to WAG 2 RI investigators in a usable format.

Data validation for DQO Levels I and II will follow ERD's requirements for quality control of analytical data. Data validation for Levels III and IV will be performed according to EPA's functional guidelines. Because no raw data is provided with Level III QC documentation, the functional guidelines which require the review of raw data will be omitted for all Level III analyses.

\subsubsection{Field data reduction and evaluation}

Data collected during field activities will be evaluated by checking the procedures used and comparing the data to previous measurements. The QAVCC coordinator and sampling team leaders will be responsible for checking field $Q C$ sample results to ensure that field measurement and sampling protocols have been observed. These reviews will check:

- date and time sampled,

- preservation,

- standard operating procedures utilized,

- calibration method and frequency, and

- chain-of-custody documentation.

Reviewers are responsible for ensuring that data reduction calculations are documented and checked by qualified personnel. Written reports, including reduced and summarized data, will include the raw data in appendices. Specific calculations used for data reduction will also be included. 


\subsection{Analytical laboratory data reduction and evaluation}

Analytical data generated during the sampling and analysis phase will be evaluated for completeness as an ongoing and concurrent process. This will include, but is not limited to, review of completed custody logs, photocopied pages of laboratory notebooks and data forms completed by the technical staff, including sample weights, dilutions, concentrations, data reduction, instrument logs, and all raw data. Reviewers of materials will include the S\&A Team Leader, WAG 2 RI QAVQC coordinator and the assigned contract laboratory program manager. In the data review process, the data are compared to information such as the sample history, sample preparation, and QC sample data to evaluate the validity of the results. Data validation includes

- dated and signed entries by technical staff and supervisors on the worksheets and logbooks used for samples;

- use of sample tracking and numbering systems to track the progress of samples through the laboratory;

- use of quality control criteria to reject or accept specific data in accordance with EPA CLP protocols and laboratory data validation functional guidelines for evaluating organic and inorganic data (EPA 1988b, EPA 1984a); and

- examination of all data for a sample and site by evaluating ion balance, checking for consistency among replicate samples, sending split samples to other laboratories for analysis, and using frequency distributions and range checks to evaluate outliers.

\subsubsection{Data reporting of analytical results}

The format and content of hard copy and electronic data reports will adhere to project needs and will be specified in SOWs. These include contract requirements of DOE and regulatory agency reporting formats. The laboratory supervisors are responsible for the preparation of each technical report, including the process of data validation. The required hardcopy report format will be specified in the laboratory statement of work.

- Final data presentation shall be checked according to data validation requirements and approved by the appropriate sampling team leader and laboratory manager.

- Each page of data will be identified with the project number or project name, sample delivery group number, batch number, and date of issue.

- Electronic copies of the data must match the hard copy reports.

Electronic data contents in the report will include:

- sample identification number used by the laboratory and/or the sample identification provided to the laboratory, if different than that used in the laboratory;

- sample delivery group number and batch number;

- chemical parameters analyzed, reported values, laboratory data qualifiers, and units of measurement;

- quantification limit of the analytical procedure;

- results of QC sample analysis;

- achieved accuracy, precision, and completeness of data;

- references to specific data if required to explain reported values; and 
- analytical methods used.

These methods will be specifically referenced on all laboratory reports. Any method modification will be included in the case narrative, provided by the contract laboratory. Data for field QC samples will be reported in the same format as actual samples.

A statistical evaluation of laboratory analytical results will be performed that will apply precision and accuracy criteria for each parameter that is analyzed. When the analysis of a sample set is completed, the QC data generated will be reviewed and evaluated as a part of validation procedures. All QC data will be reported to the WAG 2 RI project manager along with the sample analysis results. The $\mathrm{QC}$ data will be reviewed for precision and accuracy.

\subsection{Internal QC Chechs and Frequency}

Internal $Q C$ procedures involve review of the documentation of maintenance and operational procedures and inspection of the instruments and equipment by personnel other than instrument users. Inspection entails the spot inspections conducted by the QA specialist in combination with the continuous process of inspection conducted by the sampling tean leaders and laboratory supervisors as part of normal procedures.

Achieving the highest level of documentation quality is imperative to $Q C$. Bound notebooks with numbered pages will be used to record all events and activities. Data forms will be used to record selected field and laboratory measurements. All notebooks and data forms will be subject to custody recuirements including limited distribution, secure storage, and long-term retention ( 30 years after completion of sample collection and analysis).

\subsection{QA Reports to Management}

The active participation of management in WAG $2 \mathrm{RI}$ is fundamental to the success of this QA/QC Plan. Management will be aware of project activities and will participate in development, review, and operation of the project. Management will be informed of QA status and activities through the receipt, review, and/or approval of

- regular Environmental Sciences Division quality status reports,

- laboratory and project-specific QAVQC plans and procedures,

- postaudit reports and audit closures,

- surveillance reports,

- corrective-action overdue notices, and

- nonconformance reports.

Copies of these reports will be distributed to appropriate Energy Systems and Oak Ridge Field Office management and regulatory agencies, In addition, periodic assessment of QAVQC activities and data precision, accuracy, representativeness, completeness, and comparability will be conducted and reported by the QA/QC coordinator. 
Project management will inform the QA specialist, as appropriate, of the QA status of the project, especially any significant quality accomplishments. WAG $2 \mathrm{RI}$ personnel are required to inform the project manager or project support staff of all nonconformances or quality failures (Roberson and Logsdon 1989). The project manager will document and immediately report any nonconformance or quality failure to the QA specialist. It is the responsibility of the ER QA specialist to report all quality-associated activities to the ORNL quality assurance manager. 


\title{
3. SEDIMENT SAMPLING PLAN
}

\author{
H. L. Boston, T. A. Fontaine, T. L. Ashwood, and S. Y. Lee
}

\subsection{INTRODUCTION}

Contaminated sediments will be a main focus of the WAG 2 project during the first 2 years because aquatic and floodplain sediments are the largest pool of contaminants in WAG 2, external exposure to radionuclides in sediments is the primary human health risk, and transport of sediments is the most significant pathway for contaminant transport off-site. Currently, we do not have sufficient information to (1) determine if interim corrective measures are needed to protect the public and environment from exposure to contaminated sediments in WAG 2, (2) quantify sources of continued contaminant input to WAG 2, (3) quantify the inventory of key contaminants in the soils and sediments of WAG 2, (4) document contaminant transport within and out of WAG 2, and (5) predict contaminant releases and determine error bounds under future land use and/or extreme hydrologic conditions. This information is required to effectively manage contaminated sediments, to accurately assess current and potential risks, and to help guide remedial efforts in the WOC watershed. This component relies on hydrological data provided by the ERMA program (Clapp et al. 1991) and will interact with RI/FSs in other WAGs to evaluate the transport of contaminated sediments from specific source areas to the Clinch River.

As noted previously, the contaminants of greatest concern in WAG 2 are particle reactive and accumulate in floodplain soils and aquatic sediments. Table 3.1 shows a radionuclide budget for WOI. In this budget, the sediment accounts for almost the entire inventory of ${ }^{137} \mathrm{Cs}$ and ${ }^{60} \mathrm{Co}$. Even the inventory for ${ }^{90} \mathrm{Sr}$, which is not especially particle reactive, is dominated by the sediment; however, ${ }^{90} \mathrm{Sr}$ fluxes are dominated by the dissolved phase. WOL and WOCE sediments have been addressed in a number of investigations. The results of some of the more recent (after 1979) sampling campaigns are presented in ORNL 1990, Sect. 6.5. Few data are available for nonradiological contaminants in WOL. Recently, sediments in WOCE were intensively sampled by the CRRI Program as part of a CERCLA removal action to control contaminated sediments (Blaylock et al., 1991a).

Upgradient of WOL, Cerling and Spalding (1981) surveyed streambed gravels at 412 sites for ${ }^{137} \mathrm{Cs},{ }^{60} \mathrm{Co}$, and ${ }^{90} \mathrm{Sr}$. These data for gravels provide good historical information for the distribution of contaminants. Later, Cerling (1986) placed uncontaminated gravels (2- to 3.35-mm size) at selected locations in the WOC drainage and then retrieved and analyzed these gravels to identify active vs residual sources of radionuclides and metals. With the exception of recent data for the major weirs in WAG 2 (see Blaylock et al. 1991b), we have little information on nonradiological contaminants or radiological contaminants in all sediment size fractions or whole samples. Therefore, we do not have inventories of contaminants in the stream sediments. Although sediment contaminant inventories are sure to be spatially and temporally variable, they are useful because they represent potential sources of exposure or release to offsite areas.

Few data are available for floodplain soils [see ORNL 1990, Sect. 6.1 and Blaylock et al. (1991b)]. Recent data (J. Ghiron, ORNL, personal communication to H. L. Boston, ORNL, 
Table 3.1. Estimated inventories of ${ }^{137} \mathrm{Ca},{ }^{10} \mathrm{Co}$, and ${ }^{90} \mathrm{Sr}$ in the abiotic and biotic compartments of White Oak Lake

\begin{tabular}{lccc}
\hline \multicolumn{1}{c}{ Compartment } & ${ }^{137} \mathrm{Cs}$ & ${ }^{10} \mathrm{Co}$ & ${ }^{80} \mathrm{Sr}$ \\
& $(\mathrm{Bq})$ & $(\mathrm{Bq})$ & $(\mathrm{Bq})$ \\
Fish & $1.1 \times 10^{4}$ & $9.6 \times 10^{4}$ & $1.1 \times 10^{6}$ \\
Benthic Invertebrates & $4.2 \times 10^{5}$ & $9.7 \times 10^{4}$ & $\mathrm{NA}^{4}$ \\
Emergent Macrophytes & $8.4 \times 10^{6}$ & $1.1 \times 10^{6}$ & $2.3 \times 10^{7}$ \\
Sediment & $1.5 \times 10^{13}$ & $4.2 \times 10^{11}$ & $6.6 \times 10^{11}$ \\
Water & $1.7 \times 10^{8}$ & $1.3 \times 10^{7}$ & $2.2 \times 10^{8}$ \\
& $1.5 \times 10^{13}$ & $4.2 \times 10^{11}$ & $6.6 \times 10^{11}$ \\
& $(405 \mathrm{Ci})$ & $(11.5 \mathrm{Ci})$ & $(17.8 \mathrm{Ci})$ \\
\hline
\end{tabular}

"NA = not available.

Source: Loar 1989. 
August 1991) for the WOL floodplain below the confluence of WOC and Melton Branch found activities of ${ }^{137} \mathrm{Cs}$ and ${ }^{60} \mathrm{Co}$ on the order of $10^{3}$ and $10^{2} \mathrm{pCi} / \mathrm{g}$ dry soil, respectively, in the upper 5 to $10 \mathrm{in}$. Similar levels of ${ }^{137} \mathrm{Cs}$ have been found in the old interinediate pond area (ORNL 1990, Sect 6.1.2). Few data are available for soil contaminants in other areas of WAG 2.

Contaminants associated with sediments are mobilized and transported with sediments during high-discharge events. Data from Oakes et al. (1982) (Fig. 3.1) show discharge, sediment transport, and ${ }^{137} \mathrm{Cs}$ flux over WOL dam during a small flood (recurrence interval of about 1.5 year) in 1979. Data in Solomon et al. (1991) show that contaminant fluxes through WAG 2 increase greatly during storms (see also Sect. 4 of this report). The current discharge monitoring system was not designed to quantify contaminant flux during floods, although storm transport accounts for most of the sediment and sediment-associated contaminant transport. Currently, data limitations for watershed-level processes do not allow us to predict contaminant transport during moderate to extreme floods and/or under future land use conditions. Thus, sampling for contaminant transport during high flow events and developing models to predict the transport of contaminants associated with sediments are important components of the WAG 2 RI project.

\subsection{OBJECTIVES AND APPROACH}

We need to determine if hot spots (discrete areas of high contamination) exist that require interim corrective measures. We also need information on contaminant inputs to WAG 2, contaminant inventories in WAG 2 sediments, and contaminant releases from WAG 2 to off-site areas. These data will allow us to (1) help direct remedial efforts, (2) document releases of contaminated sediment from WAG 2 to demonstrate that remedial efforts have been successful, and (3) develop models that can predict the release of contaminated sediments from WAG 2 under future land use and extreme hydrologic conditions (i.e., extreme floods). Note that the WAG 2 efforts to quantify, monitor, and model contaminant fluxes will include several sites outside WAG 2 to provide information for the entire WOC drainage system. A watershed-level perspective is necessary to evaluate and predict sediment transport.

Efforts during the first 2 years of environmental monitoring will emphasize the collection of screening-level information for contaminants in floodplain soils and stream sediments, as well as initially quantifying contaminant fluxes for individual reaches in the WOC drainage system. Data for sediment-associated contaminants collected during the first 2 years of this effort will emphasize radionuclides $\left({ }^{137} \mathrm{Cs},{ }^{60} \mathrm{Co}\right.$, and $\left.{ }^{90} \mathrm{Sr}\right)$. Radionuclides are emphasized because they are important contaminants and are easy to measure. Fewer samples will be collected for metals and organic contaminants. These efforts will serve as a basis for future, mote intensive efforts. We will not address WOL or WOCE at this time because they have been fairly well characterized compared with other areas of the drainage system. However, we recognize that the large radiological inventory in WOL and the lack of data appropriate for baseline risk assessment will necessitate investigations in the future. In general, the objectives of these early efforts are to screen for contaminants in areas where few data exist, provide data to develop more intensive sampling, develop sampling techniques, and implement the multimedia environmental monitoring efforts. This sampling also supports the development of models to predict the transport of contaminants associated with sediments that are released to off-site areas during high-discharge events. 


\section{Discharge Over White Oak Dam}
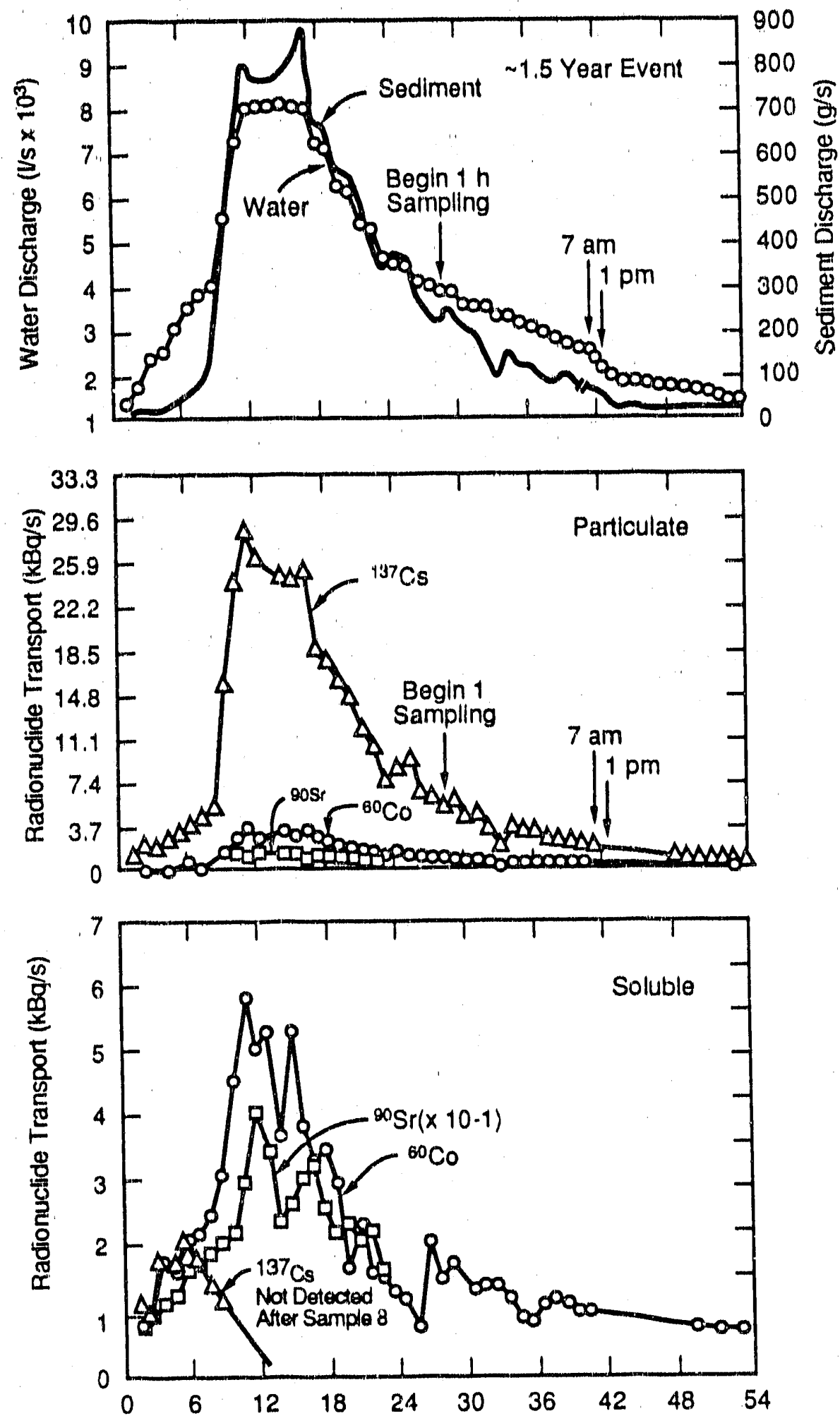

Fig. 3.1. Sediment and contaminant transport cover White Oak Dam 
The following sections will describe four objectives (with subcomponents) and the sampling and analyses to support these objectives. These objectives are

(1) conducting floodplain radiological walkover and stream sediment surveys,

(2) sampling contaminant transport during storms,

(3) quantifying contaminant inventories in stream sediments and identifying contaminant sources, and

(4) developing models to predict sediment transport for the WOC watershed.

\subsection{Objective 1-Floodplain Radiological Walkover and Stream Sediment Surveya}

Note: This activity will be carried out prior to formal review of this document.

Details of this activity have been described in communications with EPA and TDEC and will be conducted only with their full concurrence. This preliminary activity is warranted by the need to conduct the walkover during the winter when the vegetation is low and will not interfere with the survey.

1. Radiological walkover. The risk-based contaminant screening for the WOCE and results of the preliminary contaminant screening for the remainder of WAG 2 found ${ }^{137} \mathrm{Cs}$ in aquatic and floodplain sediments to be the primary contributor to human health risk. Chemrad, Inc. will conduct a scoping walkover of the White Oak Creek floodplain will be conducted using USRADS (Ultrasonic Ranging and Data System). USRADS will provide real-time data for gamma radiation to initially estimate the extent of soil and sediment contamination, to locate and define hot spots (discrete areas of high activity), and to provide preliminary information for gamma contamination for major areas of the WAG 2 floodplain for which no information currently exists. These data will form the basis of the preliminary soil sampling efforts, stratification of the system for future efforts, as well as locating areas that may require interim corrective measures.

2. Floodplain soil sampling. In addition to providing information for the surface gamma activity, the USRADS data will be used to allocate a limited number of sediment samples among locations in the floodplain. The information generated by this effort will be used to (1) provide some preliminary information on metals and organics, (2) determine if the distribution of radionuclides has a strong positive correlation with those for metals and organics (i.e., if co-contamination is common), and (3) determine the vertical distribution of contaminants in the upper $0.5 \mathrm{~m}$ of sediment. If, as expected, metal and organic contaminants accumulate in the same areas as gamma-emitting radionuclides, then the radiological surveys can be used to apportion later sampling efforts for site characterization. Furthermore, this survey will provide data needed to determine if there is a need for interim corrective measures.

3. Preliminary stream zediment sampling. Although there are significant sources of gamma-emitting radionuclides in the Melton Branch section of WAG 2, we do not expect to find widespread gamma contamination in the floodplain of the Melton Branch tributary of the WOC watershed. Tritium is an important beta contaminant in Melton Branch but is water soluble and will be accounted tor in other components of this S\&A 
plan. Strontium-90 is another important beta-emitting contaminant in the Melton Branch section of WAG 2. However, ${ }^{90} \mathrm{Sr}$ is fairly soluble, and where it does accumulate in soils and sediments, it is not easily dotectable by fleld instruments. In large areas without gatnma contamination, random or blanket sampling for contaminants without probable cause is not likely to have a high return for effort invested at this preliminary stage of the WAO 2 investigation. To determine if there is probable cause to sample in areas with little gamma contamination, we will continue to review new and existing information for facilities in the Melton Branch tributary (e.g., data generated by the WAG $5 \mathrm{RI}$ ) and will collect stream sediment samples at the major weirs in the WOC watershed. The aquatic sediments act as an integrator of contaminants in upgradient areas. The analysis of samples collected from the major weirs will provide information for contaminants in areas of the watershed upgradient of those weirs. This information will be used to screen for additional contaminants of concern in areas for which no data are available and in which significant surface gamma activity cannot be used as an indication of contaminant accumulation areas. If nonradiological contaminants are found at the weirs, those constituents will be evaluated for inclusion in the list of contaminants of concern and we will evaluate the need for modifications in the S\&A plan.

Data from stream sediment sampling in WAGs 1,5, and 6 will be used to augment these efforts. These data will be provided by the WAGs 1,5 , and $6 R^{?}$ ? projects and further augmented by WAG 2 efforts where needed.

\section{Sampling and Analysis for Objective 1}

1. Radiological walkover. The walkover will be conducted using a gamma scintillation counter coupled to the USRADS. This system permits essentially $100 \%$ coverage of the area in a real-time mode. The USRADS provides horizontal positioning control from known benchmarks and recurds data from the scintillometer at 1-s intervals. Scintillometer data is reported in counts per minute (cpm). Anomalies (readings substantially above background for the immediate area being surveyed) will be reevaluated on the spot to minimize the chance of obtaining erroneous data. Also, anomalous areas above a specified level will be flagged immediately for later sampling.

The walkover will be conducted during the winter months to avoid dense understory that interferes with both the movement of the surveyor and the ultrasonic signal. We will survey the entire WAG 2 area, including some overlap with the boundary areas of source WAGs. We will merge our information with similar information generated by walkovers of the source WAGs to form a radiological survey map of the entire watershed. Data are currently available from WAGs 1 and 6 and will soon be available from WAG 5.

2. Floodplain soil sampling. The walkover survey will provide a gamma radiation survey map of the WCC floodplain in units of cpm. To provide more useful data (i.e., activities of specific isotopes and exposure levels) for anomalously high areas, it is necessary to collect and analyze samples of the soil. Methods for the collection and analysis of soil samples are presented in Sect. 3.3. Any areas where the USRADS measurements exceed the dose rate or cpms in the immediate vicinity by $>300 \%$ will be flagged in the field by the USRADS surveyor and sampled. In areas where the anomaly covers a large area, one sample will be collected for every $2500 \mathrm{~m}^{2}$ (i.e., $50 \mathrm{~m}$ 
by $50 \mathrm{~m}$ ). A sampling intensity of one sample per $2500 \mathrm{~m}^{2}$ for contaminated areas with no distinct "hot spots" was selected to provide a cost-effective, initial estimate of variability. All samples will be collected by shallow hand-augering to a depth of 30 to $50 \mathrm{~cm}$, depending on the nature of the soil. One half of the samples will be composited over the entire depth. The other half (chosen randomly) of the samples will be sectioned into the top $5-\mathrm{cm}$ and $10-\mathrm{cm}$ intervals thereafter. All samples will be gamma counted for 100 min and up to 100 samples (including 10\% duplicates) will be analyzed for ${ }^{90} \mathrm{Sr}$. Twenty samples (including $10 \%$ duplicates) chosen randomly in the vicinity of SWSA 5 and the intermediate pond will be analyzed for transuranics (i.e., ${ }^{211} \mathrm{Am},{ }^{24} \mathrm{Cm}$, and ${ }^{22 x} \mathrm{Pu}$ ). The area immediately downgradient of SWSA 5 will be stratified into 20 equal area units, and a sample will be collected from a randomly. chosen point within each unit. Samples for ${ }^{90} \mathrm{Sr}$ will be selected based on bremsstrahlung radiation observed during the gamma counting. Thirty of the samples will be selected at random and analyzed for particle-size distribution by sieving and settling to the size class. All size fractions will be analyzed for gamma-emitting radionuclides.

From the samples (including both composites and sectioned samples), we will randomly choose 40 samples (including 10\% duplicates) to analyze for PCBs, chlordane, and metals (ICP and mercury). PCBs are the greatest contributor among organic compounds to human-health risk and ecological risk. Chlordane from an area above WAG 1 is known to accumulate in biota in WAG 2 (ORNL 1990). We will also randomly choose ten sites where the USRADS data indicate no anomalous radioactivity (by stratifying the area into 10 equal area units and randomly selecting a point within each unit) and collect 30-cm-deep composite samples. These data will be used to test the hypothesis that organics and metals, when present at all, are co-contaminants with radioisotopes. In other words, the same processes that cause deposition of the particle reactive radionuclides also cause deposition of particle reactive nonradiological contaminants. The total number of samples $(50)$ was limited by costs. However, these data will provide information for variability in the distribution of metals and organic contaminants needed to design sampling efforts to reduce uncertainty for risk estimates.

3. Preliminary stream sediment sampling. Sediment at the two lower weirs on WOC, the two lower weirs on Melton Branch, and WOC just upstream of WOL (Fig. 3.2) will be sampled for contaminants. Methods for the collection and analysis of sediment samples are presented in Sect. 3.3. We will collect two cores (up to 100-cm depth) with a hand held corer from pools at each site and section each core into the upper $10 \mathrm{~cm}$ and the remaining depth. All samples will be homogenized and analyzed for gamma emitting radionuclides, ${ }^{90} \mathrm{Sr}$, ICP metals, $\mathrm{Hg}$, semivolatile organics, PCBs, and pesticides. In addition, the upper $10 \mathrm{~cm}$ of each core will be analyzed for ${ }^{201} \mathrm{Am}$, ${ }^{24} \mathrm{Cm}$, and ${ }^{2 x} \mathrm{Pu}$. A total of 20 samples will be analyzed.

\subsubsection{Objective 2-Sampling of Contaminant Transport During Storms}

The existing surface water monitoring programs are inadequate for determining contaminant mass flux into, through, and out of WAG 2 during high flow events. The inability to obtain accurate estimates of contaminant flux results from the inadequate 


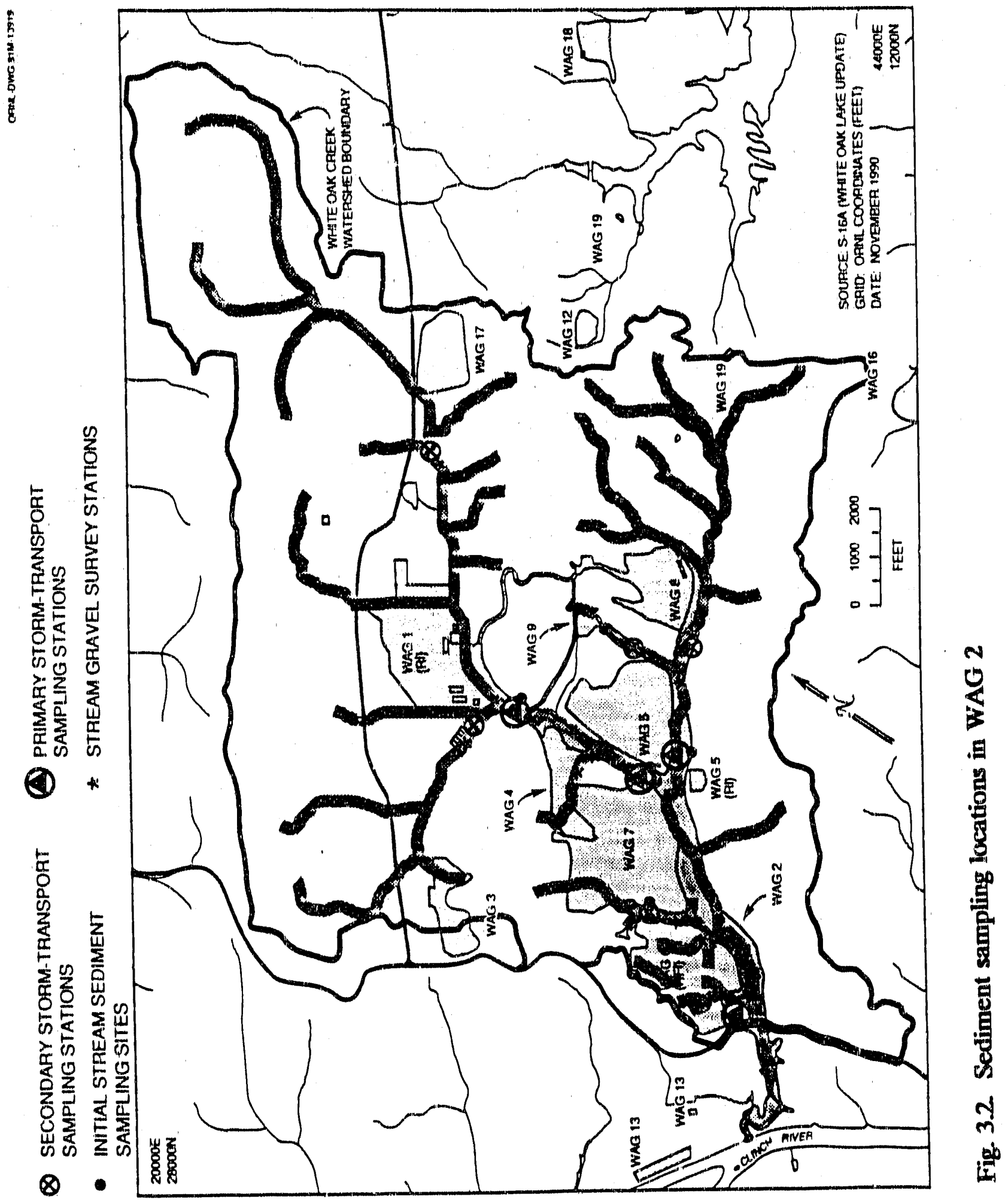


estimation of discharge during large events and inadequate sampling of suspended sediment transport (see Borders et al. 1991).

We will characterize (particle-size distribution and discharge-sediment load) and quantify sediment and contaminant transport at key points (Fig. 3.2) in the WOC system to allow a mass balance for contaminants for major reaches of the WOC watershed. These sampling, characterization, and quantification activities will allow us to determine inputs (i.e., sources), accumulation (relating to future risk), and transport for specific reaches, as well as providing a record of contaminant release from WAG 2. These data will also provide information to be used in the sediment transport modeling effort (Objective 4).

Data provided by this effort will also be used by the ERMA watershed hydrology program (Clapp et al. 1991) to evaluate the data being collected by the existing compliance monitoring efforts, and if necessary, calibration and/or modifications of existing sampling efforts will be discussed with the programs responsible. Throughout this process we will be cooperating with the Environmental Restoration Hydrologic Monitoring Program and the ORNL Environmental Monitoring and Surveillance Division to calibrate or modify the existing systems to better characterize contaminant transport during high-discharge events.

\subsection{Sampling and Analysis for Objective 2}

1. Sampling of sediment transport during storms. The concentrations of suspended sediment and bedload will be measured during events representing a range of discharges at each of seven sites in WOC. Methods for the collection and analysis of sediment samples are presented in Sect. 3.3. Four of these sites will be primary monitoring stations while the remaining three sites will be secondary monitoring stations. The primary stations will be located near the major upstream and downstream boundaries of WAG 2 [the 7500 Bridge [near USGS No. 03536550 (GS3)] and WOD (near MS5), respectively], and just upstream of the confluence of the WOC and Melton Branch tributaries (near MS3 and MS4) (Fig. 3.2) the secondary stations will be used to monitor inputs to WAG 2 on a short-term basis. Each secondary station will be maintained at a site just long enough to sample the flux of contaminants and sediment during a small flood (e.g., a 1- or 2-year event). After such a flood has been sampled, the secondary stations will be relocated to other sites. The initial sites to be sampled with secondary monitoring stations are near the West Seep, the Northwest Tributary, WAG 17 [USGS No. 03536320 (GS6)], and near MB2 and HRT in the Melton Branch Tributary (see Fig. 4.1). We will ccordinate with the Clinch River RI program and may add a sampling station at the mouth of WOCE following the completion of the sediment-retention structure planned for that site.

Storm hydrographs rise and fall quickly in WOC because the watershed is small, and runoff is flashy. Therefore, suspended sediment samples will be collected with programmable automatic sampling equipment. When stream stage exceeds a predetermined elevation, periodic samples of stream water and suspended sediment are pumped from a fixed point in the stream channel into 1-L bottles. The sampling frequency is determined by the time to peak and the rate of recession of discharge. For example, intense summer thunderstorms may require samples collected every 5 min to accurately measure changing sediment concentrations during the rise of the hydrograph, 
while 20-min intervals may be sufficient during less intense winter storms. Less frequent sampling is required during the recession of streamflow.

Samples of bedload will be collected during storms using bedload samplers (e.g., the Helley-Smith bedload sampler; Emmett 1981) and bedload traps. Bedload (coarser sediment moving with periodic contact with the streambed) will also be calculated using one or more bedload formulas (ASCE 1975) and samples of bed material (stationary material of the streambed).

The modeling efforts (Objective 4) will require samples from about three large winter storms and two large summer storms. Since sampling devices automatically activate at predetermined stages, samples will often be collected during floods that turn out to be smaller than the five largest events selected for the extensive laboratory analysis work for data required for the modeling. Thus, we will be collecting many more samples than are needed for the modeling effort. Some samples from these smaller storms will be analyzed for total suspended load. These data will be used in conjunction with the stage discharge relationships to estimate contaminant fluxes.

Only a fraction of the total number of samples collected will be analyzed for physical parameters (e.g., grain size distribution and sediment concentration). Of these, more samples will be used from the rising limb of the hydrograph than the falling limb because sediment and contaminant concentrations are usually more variable during periods when stream discharge increases rapidly (Fig. 3.3). Approximately ten sediment samples per storm will be selected for physical analysis. One replicate sample per storm will be used to check the consistency of field sampling.

A subset of the samples used for physical analysis will be selected for contaminant analysis. Initially, all 10 samples per storm collected and used for physical analysis will be analyzed for gamma-emitting radionuclides using ESD standard operating procedures. During the gamma counting, samples will be screened for energetic beta emitters (e.g., ${ }^{90} \mathrm{Sr}$ ) by monitoring for bremsstrahlung radiation. Selected samples indicating high and low beta activities will be analyzed for ${ }^{90} \mathrm{Sr}$ using the standard falboratory method to provide an estimate of the range of ${ }^{90} \mathrm{Sr}$ concentrations and so estimate ${ }^{90} \mathrm{Sr}$ flux.

After samples from several storms have been analyzed for gamina and beta radionuclides and the variability of radionuclide activity associated with sediment transport during a flood is determined, the number of samples analyzed for radioactivity will be reduced to the minimum number of measurements required to estimate the transport of radioactive contamination on sediments during floods (e.g., samples during the beginning, middle, and end of a flood). A small number of samples will be selected for analyses of metallic and organic contaminants. Samples taken near the peak discharge of several floods having high gamma and beta activities will be screened for metallic contaminants. A suspended sediment sample will be taken at each monitoring station during the peak discharge of one of the largest floods in the first year of sediment monitoring and analyzed for organic contaminants. This approach will provide a sufficient number of samples to provide a first estimate of the amount and variability of radioactive, metallic and organic contamination transported with sediment during floods during the first year of the S\&A plan. Approximately 250 gamma, $100^{90} \mathrm{Sr}, 80$ metal, and 7 organic analyses will be conducted in the first year using this plan. 


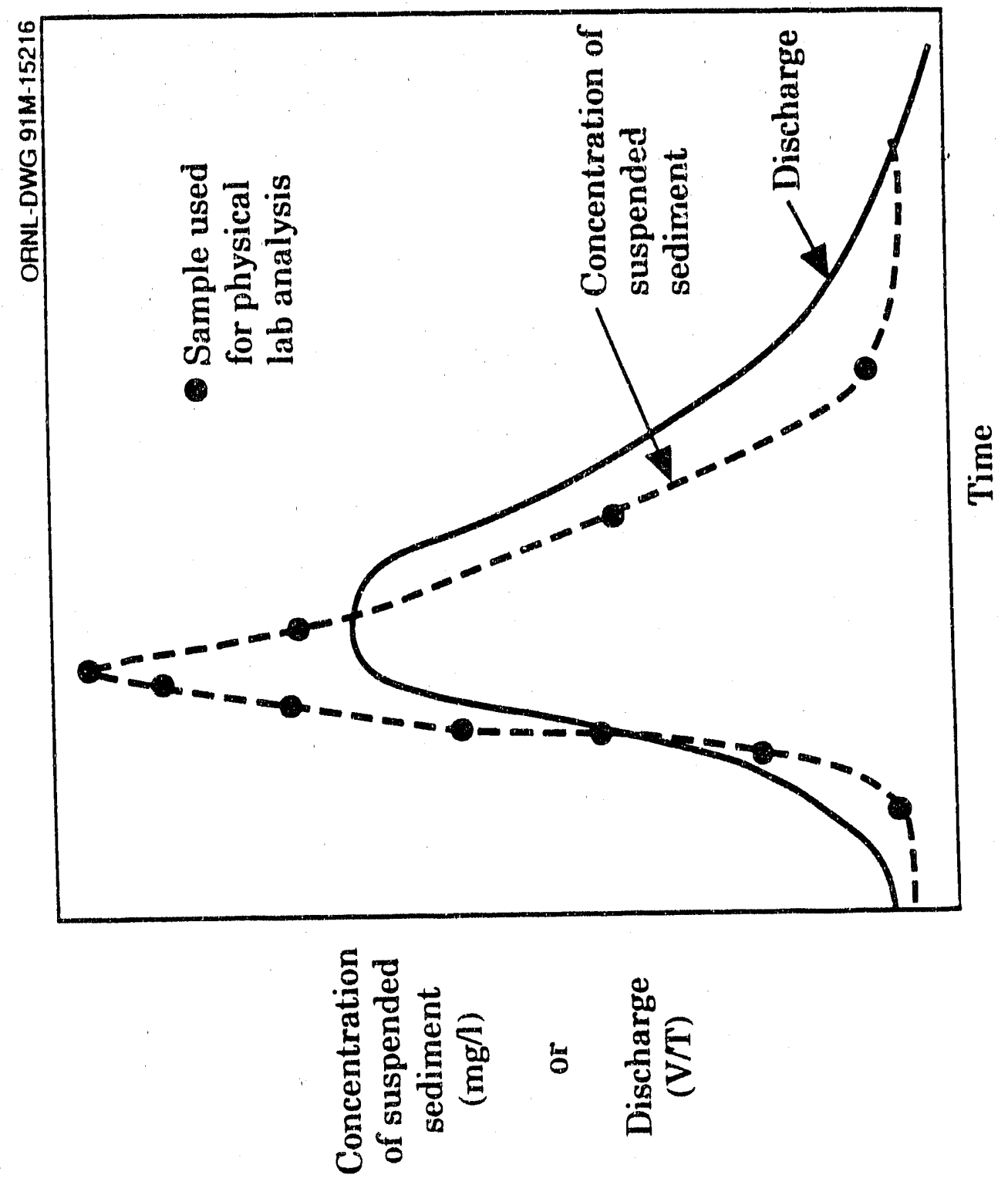

를

mi in 
The S\&A for these types of contaminants in following years may require adjustments in the number of events sampled and frequency of sampling during events to improve the reliability of the estimates based on the analyses done during the first year. One in every 20 samples analyzed for gamma, ${ }^{90} \mathrm{Sr}$, and metals will be replicated, and 1 of the 7 organic samples will be replicated.

Particle-size distributions of suspended sediment, bed material, and bedload samples will be developed by sieve and settling analyses (Guy 1969, ASCE 1975). Total sediment concentrations are used along with discharge measurements to develop sediment-discharge rating curves for each monitoring station. These data will allow an estimate of sediment and radionuclide transport at key points in the watershed to (1) identify contaminant sources, (2) quantify contaminant fluxes into and out of WAG 2 (i.e., to the Clinch River), and (3) provide data needed in the sediment transport modeling effort. In addition, these data will be useful for evaluating the existing NPDES monitoring datà.

2. Calibrating automatic samplers. The automatic samplers required for sampling suspended sediments in a small watershed withdraw the samples at a fixed point in the stream (e.g., $1 \mathrm{ft}$ above the streambed at the centerline of the channel). Because the concentration of suspended sediment will vary significantly both horizontally and vertically across the stream channel, it is necessary to calibrate the data collected by point withdrawals so that sediment flux can be estimated. Results from automatic samplers can either overestimate or underestimate the average concentration of sediment. To calibrate automatic samples and estimate variability under these circumstances, manual sampling is used to measure the average suspended sediment concentration at the same time an automatic sample is taken. Established sampling techniques described by Guy and Norman (1982), ASTM (1990) and by the American Society of Civil Engineers (ASCE 1975) will be adapted for the conditions in WOC. After concurrent automatic and manual samples have reen collected for a range of discharges, a relationship is developed to transform automatic samples taken at a single point in the channel into an average value of sediment concentration using a calibration curve.

\subsection{Objective 3-Quantifying and Tracking Contaminant Inventories in Stream Sediments and Identifying Contaminant Sources}

Evaluating contaminant inputs to and releases from WAG 2 will be complicated by the dynamics of contaminants currently in WAG 2. Areas of the drainage system behave differently with regard to contaminant dynamics. Some areas will act primarily as long-term sinks for contaminants that are being transported in WAG 2 (e.g., depositional areas in the floodplain and pools upstream of the weirs). These areas have the potential to act as contaminant source areas during extreme hydrologic events or following alterations in the watershed. Other areas will primarily act as conduits and will simply transport contaminants with little contaminant accumulation or contribution to contaminant mass flux. The following components of Objective 3 address the need to quantify and track contaminant inventories in stream sediments and to identify contaminant source areas. 
1. Distribution and inventory of radiological contaminants in stream sediments. A scopinglevel sampling of aquatic and floodplain sediments in WAG 2 was described under Objective 1 of this task. Based partly on the results of the scoping survey, additional efforts will be launched to (1) provide an initial quantification of radiological contaminants in stream sediments and (2) map the creek sediments and determine the distribution of radionuclides among sediment size classes.

The information provided will allow a preliminary quantification of contaminant inventories and provide information helpful for the design of a sampling program to evaluate nonradiological contaminants. The data collected as part of this objective will also be used to parameterize the sediment transport model (Objective 4).

2. Stream gravel survey program. Cerling and Spalding (1982), Cerling and Turner (1982), and Cerling et al. (1990) showed that the distribution of radionuclides and metals on sediments was particle-size dependent. Further, they found that ${ }^{137} \mathrm{Cs}$ was irreversibly bound to illite clays in these fractions; ${ }^{60} \mathrm{Co}$ was associated with manganese oxides and so subject to removal by changes in redox potential, abrasion, and exchange; and ${ }^{90} \mathrm{Sr}$ was associated with cation exchange sites. Gravel-sized stream sediments derived from Conasauga shale have a high illite content and were found to be useful for investigating contaminant inputs and distribution. Further, the gravel fraction accounted for a sizable portion of the stream sediment contaminant inventories in the upstream areas of the WOC system (Cerling and Spalding 1982).

Cerling (1986) proposed a monitoring program using stream gravels (2.0- to 3.3-mm size) to identify source areas for active vs residual contamination. The gravel size fraction is rich in illite clay and strongly sorbs ${ }^{137} \mathrm{Cs}$, ${ }^{60} \mathrm{Co}$, and ${ }^{90} \mathrm{Sr}$. In 1985 , clean gravel-sized sediment was collected (wet seived) from an uncontaminated upstream location on Melton Branch was placed in slotted (1-mm slots) well casings, capped, and tethered to the streambank at 17 sites associated with key branch points in the drainage system for 4 weeks. These data were useful for identifying active sources of contamination as well as estimating contaminant fluxes. An example of data from Cerling (1986) on ${ }^{90} \mathrm{Sr}$ and ${ }^{137} \mathrm{Cs}$ activities in gravels at key locations in the WOC drainage shows the differences in source areas for these contaminants (Fig. 3.4). This technique provides information for contaminants that are not easily measured in the water column and is not confounded by high discharge events that move and mix stream sediments. We propose to use this technique during 1992 and again about every 3 years as a simple and inexpensive means to monitor and track contaminant source areas during and following the remediation of the ORNL WAGs.

Although the stream gravel survey program will identify contaminant sources to surface water, because these contaminants accumulate in sediments and because the collection and analytical methods are more closely related to the sediment sampling than to the surface water sampling, the stream gravel survey is discussed under the sediment sampling plan.

3. Additional objectives for year 2. During the second or third year, we will initiate studies to determine the behavior of contaminated sediments in selected pools, riffles, and stream bank soils to aid in the development of the sediment transport model. This activity will be described in future communications. 

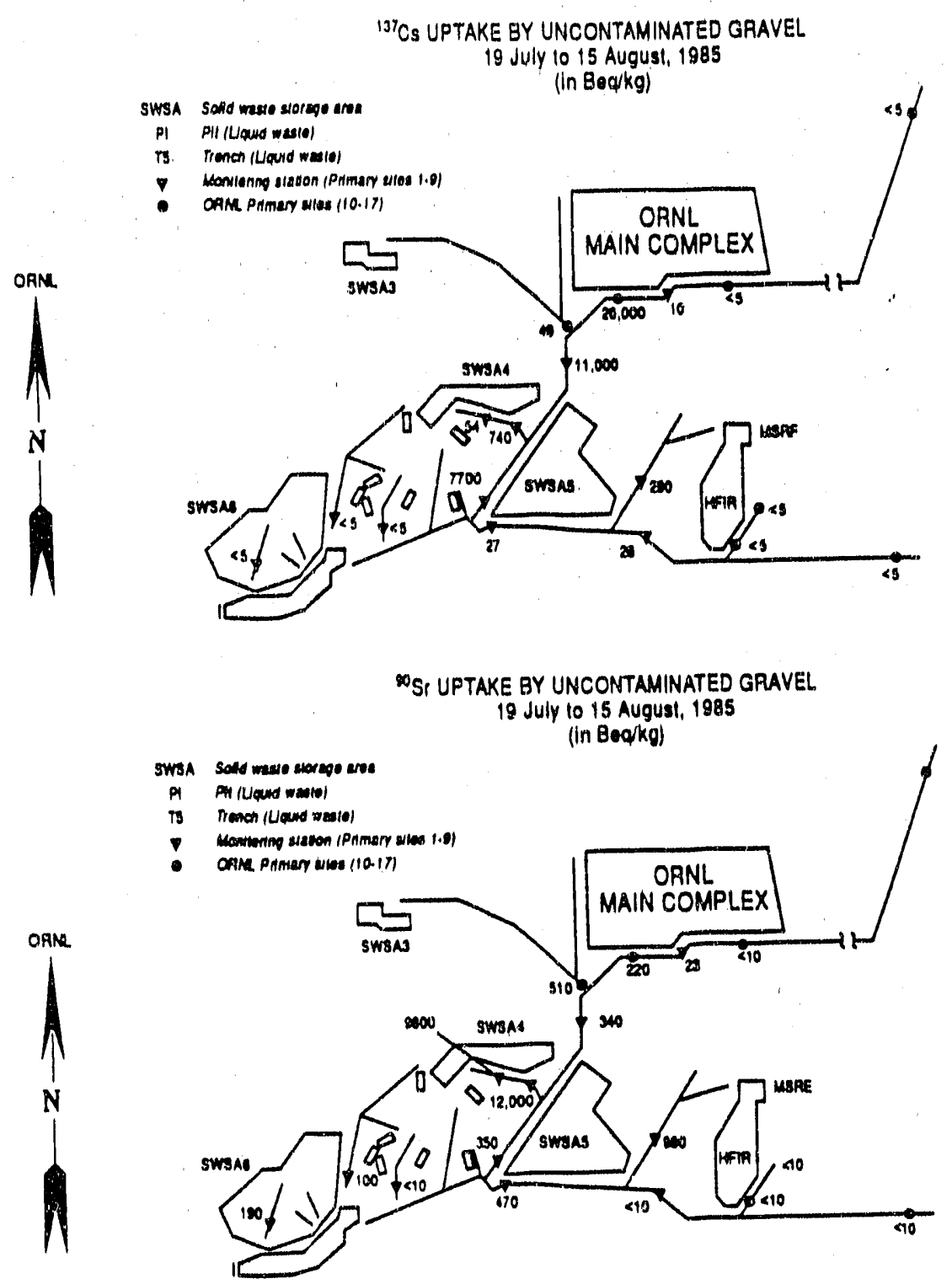

Fig. 3.4. Stream gravel ${ }^{137} \mathrm{Cs}$ and ${ }^{90} \mathrm{Sr}$ survey data sampled in 1985 


\subsection{Sampling and Analysis for Objective 3}

1. Distribution and inventory of radiological contaminants in stream sediments. Before stream sediments are surveyed, we need information on the distribution of radiological contaminants horizontally and vertically in riffles and pools. To gather this information, we will intensively survey a riffle and a pool area in the upstream area of WAG 2 and in the area just above WOL. In each area, 12 sediment samples will be collected representing different water depths and locations within the pool or riffle. Methods for the collection and analysis of sediment samples are presented in Sect. 3.3. Sediment cores will be collected in $25-\mathrm{mm}$-diam by $\mathrm{i}-\mathrm{m}$ stainless steel tubes by means of a hand-held corer. Sediment cores will be taken to the laboratory and sectioned into the upper $5 \mathrm{~cm}$, next $10 \mathrm{~cm}$, and then by $20-\mathrm{cm}$ intervals. Samples will be analyzed for gamma-emitting radionuclides with a germanium (IG) detector. Subsamples of each section will be analyzed for ${ }^{90} \mathrm{Sr}$. Following gamma analysis, the samples will be analyzed for particle size distribution by sieving and by settling. This sampling will focus on radionuclides. Samples to be analyzed for other contaminants will be collected in the future based on the results of this effort and data provided by Objectives 1 and 2 in this section.

This survey will provide information to determine the need to randomly or authoritatively (at predetermined locations) sample the sediments in the remainder of the WOC system downstream of contaminant inputs and the utility of analyzing discrete depth intervals as opposed to entire cores. Approximately 50 cores will be collected from riffle and pool areas during year 2 . The sampling locations will be apportioned to estimate inventories for key reaches and to extrapolate to the remainder of the system. Samples will also be collected from tributaries near the confluence with WOC or Melton Branch to evaluate potential contaminant inputs from those areas. This process will be repeated every 2 to 4 years to update the contaminant inventories as upgradient WAGs are remediated.

To extrapolate from the sediment sampling data to the entire stream area downstream of contaminant inputs, the streams will be mapped by using tapes and depth-measuring sticks. Eack pool and riffle area will be numbered and its location established relative to permanent markers on the stream bank and entered into our Geographical Information System data base. Data for the stream width, water depth, bank height, and composition will be recorded. These data will also be needed in the sediment transport modeling efforts.

2. Stream gravel survey program. Following the methods of Cerling (1986), clean gravelsized (2- to $3.3-\mathrm{mm})$ sediments will be collected by wet seiving and placed in slotted 25-mm-diam well casings, capped, and tethered to the stream bank for 4 weeks at approximately 20 key locations (Fig. 3.2). Replicate casings with clean gravels will be placed at at least five of these sites to provide an estimate of the variability of the method. After 4 weeks in the field, the gravels will be analyzed for gamma-emitting radionuclides and ${ }^{90} \mathrm{Sr}$ as described for other stream sediment samples. 


\subsection{Objective 4-Develop Models to Predict Sediment Transport for the WOC Watershed}

We will develop a model capable of simulating contaminant distribution and transport with sediments to (1) predict contaminant transport during extreme events and (2) predict contaminant transport during and following corrective measures in upgradient WAGs (e.g., increased surface runoff following capping of large portions of WAG 6).

This component is intended to meet the following objectives.

1. Identify and evaluate processes controlling sediment and contaminant transport (stagedischarge-sediment flux) during moderate- to high-discharge events (Objective 2).

2. Predict contaminant transport under future conditions. We will provide a means to predict future sediment-associated contaminant transport and release for WAG 2 (i.e., how remedial actions and other modifications of the watershed will influence contaminant input and transport for WAG 2; how contaminant input, transport, and release for WAG 2 are likely to vary with natural factors such as wet years vs dry years; how to evaluate year-to-year variability in contaminant releases; how to predict transport of contaminants off-site during floods; and how to evaluate the need for interim corrective measures).

3. Provide estimates of uncertainty in model results.

4. Link models for sediment tramsport in the WOC watershed with similar efforts for the Clinch River RI (CRRI). The RIs for WAGs 1, 5, and 6 have developed or are developing models of contaminant transport. These models include sediment transport. These areas are upgradient of WAG 2; thus, it is important to be able to link information generated as part of those efforts with the WAG 2 efforts to develop a watershed-level perspective. To develop these linkages, we have met with the RFI teams for WAGs 1 , 5, and 6. All three of these groups will be using the computer model that we have selected, the Hydrologic Simulation Program-Fortran (HSPF) (EPA 1984c). The WAG 1 team has collected sediment transport data, and they will share results and information with us. The WAG 5 RI team has not yet begun; however, we have agreed to coordinate data collection and analysis. Similarly, efforts to sample and model sediment transport for WAG 6 are continuing, and we are making arrangements to cooperate with that group. The Watershed Hydrology Group is providing hydrological support for ali of these programs and so strengthens the linkage among these efforts (Clapp et al. 1991).

The WAG 2 project is also linking sediment transport efforts with the CRRI. That project is working in cooperation with the Tennessee Valley Authority (TVA) and an international model validation effort to evaluate sediment transport downstream from the Oak Ridge Reservation. The WAG 2 RI and CRRI modeling ieams share two key staff members to facilitate this coordination. Information and modeling from the WOC watershed will help the CRRI to evaluate the discharge of contaminants and sediment into the Clinch River from several catchments in the Oak Ridge Reservation (e.g., East Fork Poplar Creek, Bear Creek, and other contaminated watersheds). 
To achieve these objectives we need to select one or more computer models and collect data. The model must be complex enough to simulate all of the important physical and chemical processes involved in contaminated sediment transport during floods ranging from a 1-year to a 100-year event. In addition, it will be necessary to simulate these processes under a variety of conditions (e.g., various land uses and various sources of contaminants and sediment). The data available to calibrate the model are generally limited to small floods and the existing land use conditions and distribution of contaminant sources. Therefore, a process-oriented model with parameters that can be adjusted to match hypothetical conditions is preferred over a model that simulates transport during extreme floods based on simple extrapolation from observed conditions. A process-oriented transport model will also be useful to increase our understanding of the critical physical and chemical processes involved in this application. However, because the complexity of the model will be limited by the amount and quality of data that can be collected to construct and apply the model, a balance is required between a simple empirical model and a complex, physically based, spatially distributed model.

Model components are required for streamflow generation (rainfall-runoff processes), channel hydraulics and flood routing, sediment transport, and contaminant transport (dissolved and particle reactive, including sediment-contaminant interaction in some cases). These processes must be accurately simulated at the catchment scale for floods between the 1 -year and 100 -year evenis. The HSPF model is appropriate for this application. HSPF is capable of simulating the major physical and chemical processes involved with the transport of contaminated sediment in WOC. HSPF has sufficient complexity to simulate the response of WOC under existing conditions as well as under hypothetical conditions that could exist during and after corrective measures. At the same time, the model is simple enough that preliminary results could be obtained within several years by using a simplified approach and an intensive S\&A program (the preliminary results could be generated in a year if sufficient data were already available). The model is well-documented, and several of the staff at ORNL have used or are currently using HSPF.

The major factor in the accuracy of model results with the application of HSPF to WOC will be the amount and quality of data available for calibration (calibration is used here to mean both calibration and verification of model parameters). It is anticipated that several years of S\&A will be required to conduct the full calibration and application of HSPF for the modeling objectives listed above. However, the first 1 to 2 years of S\&A should produce enough data to begin the initial calibration, simulation, and uncertainty analysis. Initial simulation runs based on parameters estimated from literature values and expert opinion, using uncertainty analysis, will determine the subset of parameters that need to be precisely estimated to yield satisfactory confidence levels. Data collection during subsequent years will focus on larger, less frequent floods (e.g., 5- to 10-year events). The length of time between the occurrence of larger floods is difficult to predict; 10 years or more of low-level surveillance may be required before several floods in the 5- to 10-year range are monitored. As the magnitudes of observed floods increase, the uncertainty of the calibration and application results should decrease. Therefore, monitoring for larger floods may continue for the $\mathbf{1 0}$ years or more to reduce the uncertainty of model results at large discharges.

In the following section we describe the S\&A plan required assuming that HSPF will be used. The S\&A plan may be modified as the initial data are collected; the important 
hydrologic and transport processes are defined in more detail; and the initial calibration, uncertainty analysis, and application of the model are completed.

\subsubsection{Sampling and Analysis for Objective 4}

During the first 3 yeare of this effort, we will focus on identifying and evaluating important processes that determine sediment transport in the WOC watershed, improving sampling techniques, and collecting data for the preliminary calibration, uncertainty analysis, and application of the model. After the initial phase, the program will be modified to focus on the data required to reduce uncertainty in the model results, especially data collected during large floods.

Types of data to be collected include information on rainfall-runoff processes, sediment supply (e.g., erosion, scour, and deposition), sediment transport during storms, sediment-contaminant relationships, and sampling to reduce uncertainty. Specific types of data required for HSPF are listed in Appendix B. The S\&A program planned to collect these data is described in the remainder of this section.

1. Sampling of rainfall-runoff processes. Data required to model streamflow generation include precipitation, discharge hydrographs, potential evapotranspiration, soil characteristics, vegetation, topography, land use, and channel geometry. Much of this information already exists or is collected on a routine basis (Clapp et al. 1991). The ERMA Program and the Environmental Monitoring and Surveillance Division are currently collecting much of the hydrologic data (discharge, precipitation, and pan evaporation) that will be needed for the WAG 2 transport modeling. The only anticipated problem with the hydrologic data is accurate discharge measurements during large floods. The locations of existing streamflow measurement stations that are important to the WAG 2 modeling analysis include WOD, the East and West Seeps, the WOC and Melton Branch weirs, several stations upstream of the Melton Branch weir, the 7500 Bridge, and several stations upstream of the 7500 Bridge. Some of these stations need to be modified to provide accurate measurements at higher discharges. This is a current activity in the ERMA Program (Clapp et al. 1991) and is discussed in detail in Borders et al. (1991). These activities are critical to WAG 2 efforts to quantify contaminant fluxes and identify contaminant sources. In the meantime, discharge measurements will be adjusted to obtain the best possible estimate of streamflow during floods.

2. Sampling of sediment supply (erosion, scour, and deposition). Erosion of hillslopes, floodplain sediments, and stream banks and scour of streambeds are the major processes controlling sediment supply. Eroded and scoured sediment can be contaminated, adding new sources of contamination as water levels increase during larger floods. Deposition of sediments during small floods creates new areas of contamination that are potential sources of off-site contamination during larger floods in the future. The amount of gravel, sand, silt, and clay currently stored in the channel system will be measured (see Objective 3). Erosion, scour, and deposition rates of cohesive and noncohesive sediment will be correlated with intensity of rainfall, stage, and discharge by using periodic surveys at selected locations on hillslopes, floodplains, stream banks, and streambeds. Methods similar to those described in Lal (1988) and ASCE (1975) will be adapted for conditions in WOC. Also see Future Efforts (Sect. 3.4). 
3. Sampling of sexliment transport during storma. Descriptions of the sampling of suspended sediment and bedload during storms have been given in Sect. 3.2.4. Suspended sediment and bedload samples collected during 5 of the largest storms in the initial S\&A program will be analyzed for particle-size distribution, sediment concentration, and associated contamination using methods identiffed in Objective 2. Bed material samples will be used when necessary to provide data to calculate bedload using formulas from ASCE (1975). In addition, samples of bed material from each sediment monitoring station (Objective 2) will be analyzed for grain size distribution, mass transport during floods, mineralogy, and critical values of shear stress for the deposition and scour of silt and clay.

4. Sampling to define rediment-contaminant relationships. Sampling for this component will be conducted during year 2 and beyond. Chemical analyses include measurements of contaminant concentrations (correlated with particle size, mineralogy, and location of the sediment source in WOC), organic content, and measurement of particle-surface and water chemistry variables that influence sediment-contaminant interaction (e.g.: manganese or iron oxide coatings, $\mathrm{pH}$, and temperature). Contaminant loadings to surface water (dissolved and adsorbed on sediment) and to subsurface flow (dissolved and adsorbed to colloids) will be measured. Additional details of the sampling to define sediment-contaminant relationships will be developed as more information becomes available on the sources and transport mechanisms of the critical contaminants.

5. Sampling to reduce uncertainty. High uncertainty can exist in the simulation of contaminant transport for moderate to extreme floods if the model has only been calibrated for small floods. Uncertainty can be caused by incorrect model structure, errors in calibration data, and natural variability in the processes involved with floods and contaminant transport. The uncertainty analysis will estimate the uncertainty in the initial model results, identify the major sources of the uncertainty, and point to ways to reduce the uncertainty, if possible. Sensitivity analysis of the model structure and reduction of the errors in the calibration data will be an ongoing process throughout tho modeling application.

\subsection{Support from the ERMA Program}

As part of the site investigation component of the ERMA Program, stage-discharge relationships will be developed for high flows at the NPDES monitoring stations on WOC and Melton Branch. The relationships previously used at these stations were established by using scale physical models and have never been verified from field measurements. In addition, submergence of the broad-crested weir at the Melton Branch station at high flows, due to downstream channel capacity, renders the theoretical relationship invalid. The stage-discharge relationship here will be applied for verification of a theoretical extended rating at the low. flow control. Improved high-flow measurements at these primary surface water monitoring stations will aid in the development of a water budget and in the quantification of mass flux of contaminants from the two major subbasins contributing to WAG 2. Though interim corrective measures have not been determined at this time, the upgrade of these surface water monitoring weirs is essential for evaluation of the effectiveness of any measures implemented (see Borders et al. 1991).

Another task implemented under the site investigation component of the ERMA Program involves collection of continuous precipitation data ( 5 sites) and surface-water discharge data 
for stations (14 sites) in the WOC watershed. The data will be reported for the surface water hydrologic data base. An annual hydrologic data summary report for the WOC watershed for water year 1990 has been published (Borders et al. 1991). The needs of data users within the ER Program will be identified and met. All data will be processed and made available, as appropriate, for the WAG 2 characterization.

\subsection{SAMPLING AND ANALYYTICAL METHODS}

Methods for sample collection analysis are presented in Tables 3.2 and 3.3. These tables emphasize activities to be conducted during year 1. All sampling and analysis procedures will be reviewed by the ERD.Analytical Program Office (APO). The ERD.APO will coordinate external (non-Energy Systems) analytical services.

\subsection{FUTURE EFFORTS}

This determination of sediment input rate to White Oak Lake by soil erosion models and fallout cesium measurement has been identified as an activity for future inclusion in the WAG 2 effort.

The WOL has been used as a sediment settling basin for contaminated sediment from the surrounding floodplain as well as from uncontaminated soil eroded from upland of the watershed. The eroded soil particles mix with contaminated sediment in the stream water during sediment transport. The eroded soil has been and will be a major source of sediments for the lake. Therefore, the functional life of the WOL as a sediment accumulation basin will largely depend on the soil erosion rate in the watershed. The erosion rate will depend on soil properties, landscape, precipitation, and land use practices. We need to be able to predict how long the WOL can serve as a sediment-(and contaminant attached with the sediment) accumulation basin given the present erosion rate. A sensitivity analysis needs to be performed relating to how possible changes of land use practice within the watershed could shorten the functional life of WOL.

The soil erosion rate can be determined by two independent approaches; (1) water erosion prediction models and (2) fallout ${ }^{137} \mathrm{Cs}$ loss measurement. Conceptual models for the water erosion prediction approach have been developed by several different groups, all having similar input parameters. Most of the input parameters for the watershed soils will be available when a soil survey and characterization of the watershed area are completed under the ORRHAGS program. Using the fallout-cesium-loss approach, cesium redistribution at sites representing ridgetop, side-slope, foot-slope, and depositional landscape positions will be quantified throughout the watershed. Integrated ${ }^{137} \mathrm{Cs}$ loss will be converted to total soil losses or total sediment input to WOL during the next 20 years of the watershed. The results of both approaches will be compared and used for prediction of sediment accumulation rate in WOL. 


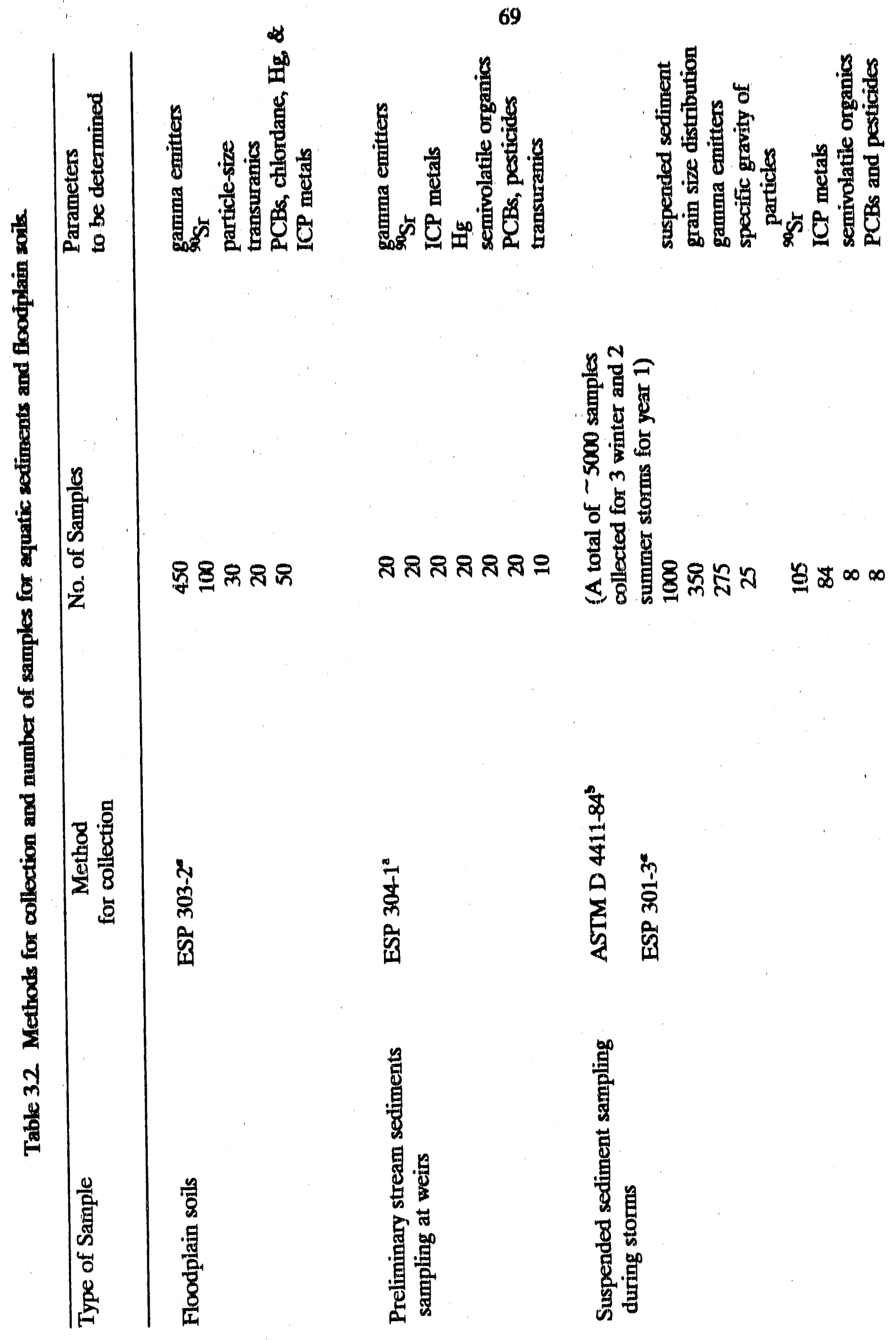




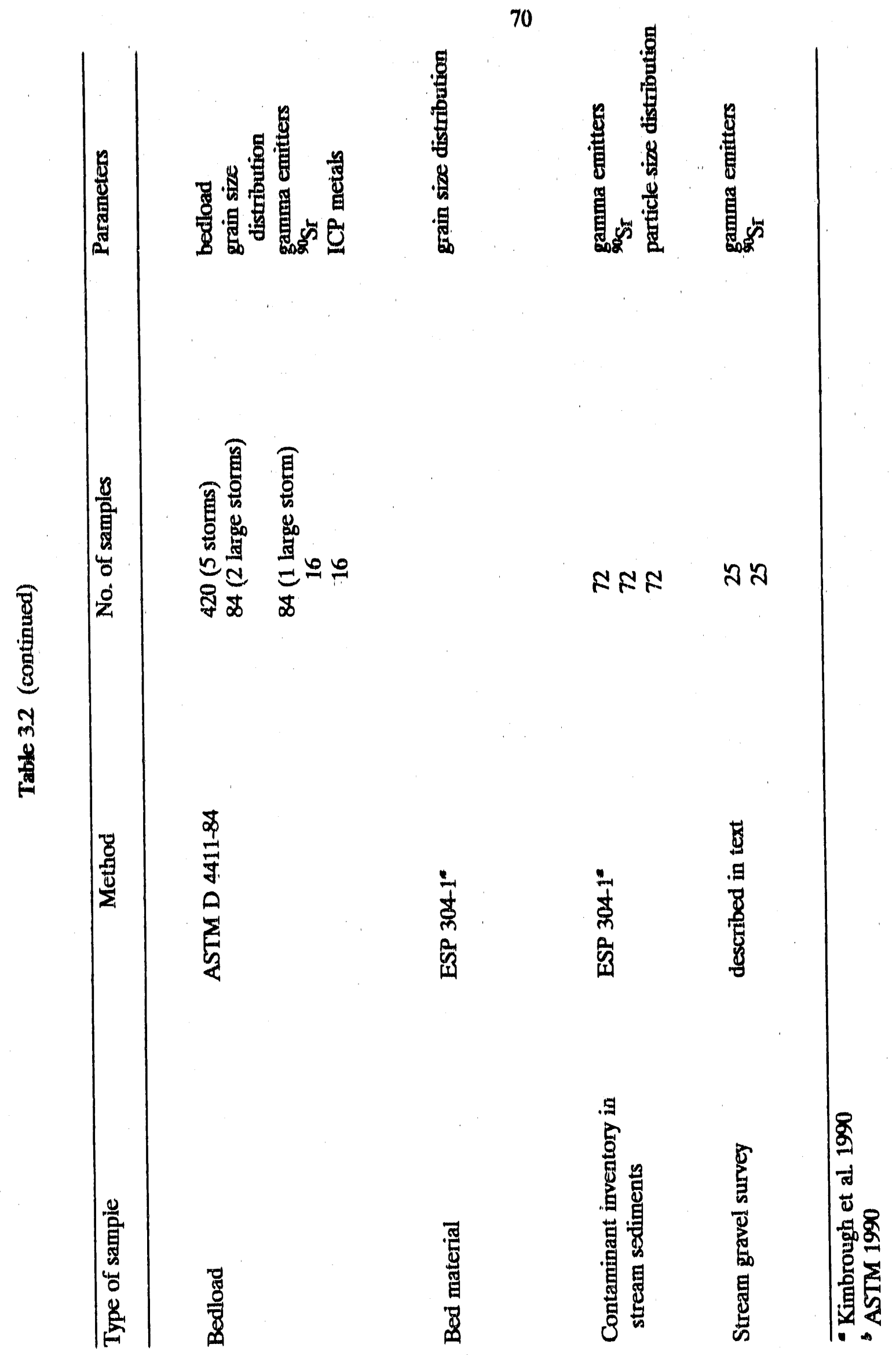




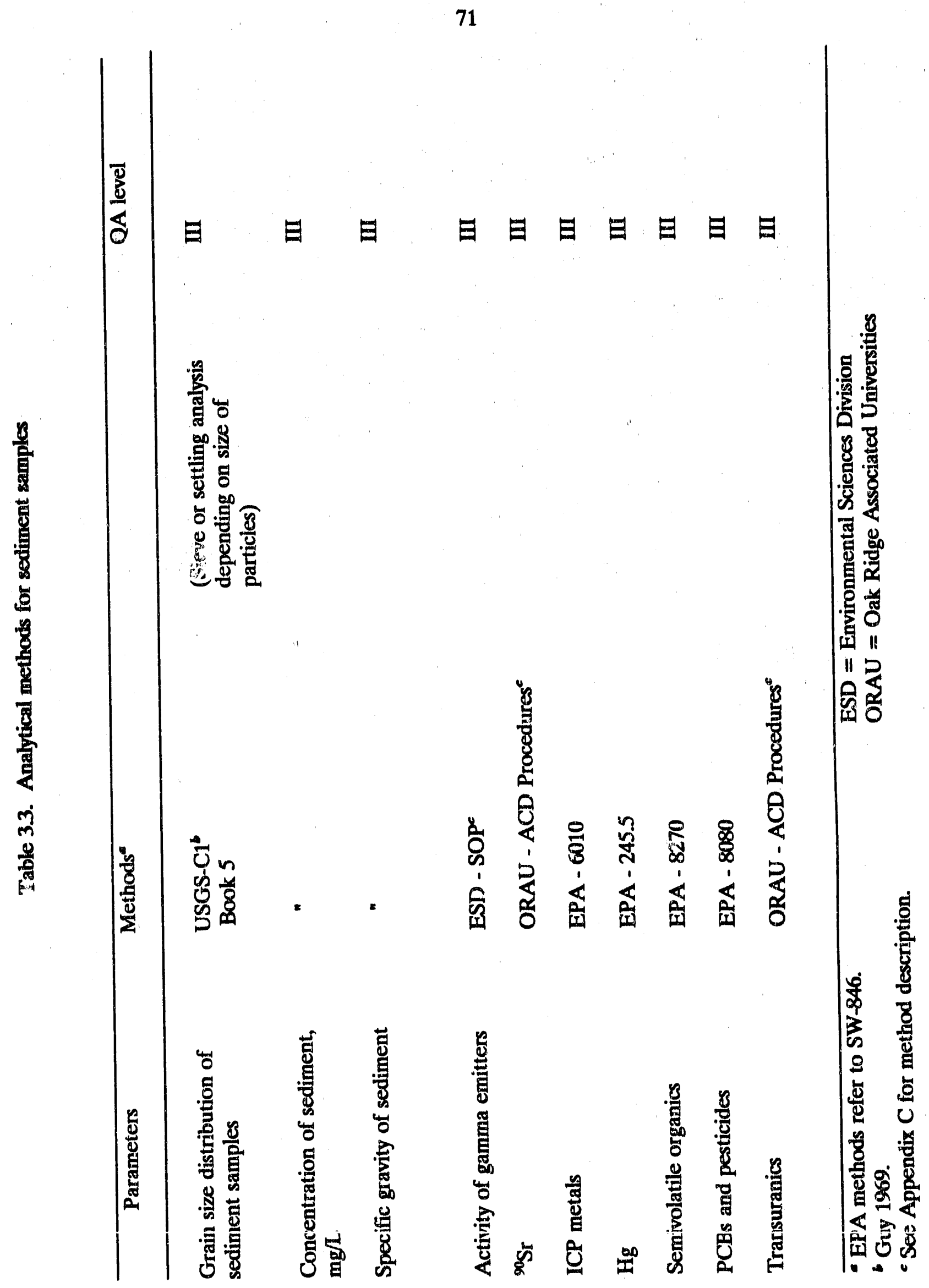




\title{
4. SURFACE WATER SAMPLING PROGRAM
}

\author{
D. S. Wickliff, D. M. Borders, and M. S. Tardiff
}

\subsection{INTRODUCTION}

Surface water is the primary transport pathway for contaminants out of the WOC watershed. There are a number of contaminants of concern in surface water (notably ${ }^{3} \mathrm{H}$, ${ }^{90} \mathrm{Sr}, \mathrm{PCBs}$, and possibly arsenic and thallium). The transport of sediment-associated contaminants is addressed in the sediment sampling plans, and thus these components are being closely coordinated. Under baseflow conditions the transport of soluble contaminants can be quantified for the major reaches of WAG 2 by data provided by two existing monitoring programs at ORNL that collect data for compliance with state and federal regulations or for compliance with DOE orders. The first objective of the surface water sampling plan is to use data generated by the compliance-driven monitoring programs to quantify contaminant fluxes (under non-storm conditions) from the major reaches of WAG 2. These efforts will be augmented in year 2 to include organic contaminants. This component supports efforts to identify sources and fluxes of contaminants into WAG 2 via seeps and tributaries.

The second objective of the surface-water sampling program is to identify seeps and tributaries that are responsible for contaminant fluxes to the main channels of WAG 2. Because greater than $95 \%$ of groundwater discharges into surface water prior to leaving the WOC watershed (D. K. Solomon, ORNL, personal communication io H. L. Boston, ORNL, August 1991), seeps (connections between groundwater and surface water) are useful for evaluating fluxes of contaminated groundwater. This component will coordinate with the groundwater efforts, and the compliance monitoring at the major weirs to identify seeps and tributaries responsible for contaminant fluxes to WAG 2, determine sources of those contaminants, develop a program to track contaminant inputs, and provide information to determine if interim corrective measures are appropriate. The tributary component will be supported by ERMA and will be linked to the WAG 2 sediment sampling efforts to evaluate contaminant transport during high-flow events.

\subsubsection{Main Channels}

The hydrologic cycle provides the main mechanisms for contaminant mobilization and transport. In WAG 2, contaminants are primarily transported by sediments suspended in surface water. However, surface water in WAG 2 would pose a significant risk to a future full-time occupant of the site as a result primarily of ${ }^{3} \mathrm{H}$ and ${ }^{90} \mathrm{Sr}$ ingestion.

Because surface water is a major transport pathway for contaminants through and out of WAG 2 to the Clinch River, understanding the surface-water hydrology and determining a water budget for WAG 2 are important for quantifying contaminant fate and transport. Contaminants carried into WAG 2 in the main branches of WOC and Melton Branch will be quantified at key control points in WAG 2 by the surface-water monitoring component. Better information for water budgets and improved sampling at existing USGS stations and NPDES monitoring points can begin to identify areas of contaminant input based on differences in mase fluxes. The stream sampling program (ORNL 1990, Sect. 9.4.1) addresses 
contaminant input from adjacent WAGs and will be developed during FY 1992 in cooperation with RFIs on other WAGs and other stream sampling programs. Many upgradient WAGs (e.g., 4, 7, 8, and 9) will not have sampling programs developed by this time. Therefore, much of the stream sampling will be initiated by this task in cooperation with the ERMA program (Clapp et al. 1991).

Contaminants associated with suspended sediment particles moving into and through WAG 2 will be quantified by the sediment transport component (Sect. 3 of this plan).

\subsubsection{Tributaries and Seeps}

Tributaries and seeps are significant pathways of contaminant input into WAG 2. Although these inputs have not been considered explicitly in the preliminary contaminant screening, investigation of tributaries and groundwater discharge areas is important for (1) identifying and quantifying sources of contaminant input into WAG 2 and (2) assessing remedial alternatives. Tributaries can serve as spatial integrators of contaminant releases from areas adjacent to WAG 2. Seeps represent connections between subsurface and surface flow regimes; therefore, they can provide insight into contaminant pathways to WAG 2 (groundwater or shallow subsurface flows) and so help to select and evaluate remedial alternatives.

\subsubsection{Review of Existing Data}

\subsubsection{Main channels}

A review of contaminant data for WOC is provided in Sect. 6 of the WAG 2 RI plan (ORNL 1990) and in each of the annual environmental reports produced by the ORNL Environmental Surveillance and Protection Section. Contaminant data are routinely generated by S\&A programs that support the objectives of compliance with the NPDES permit for the facility and compliance with the 5400 series DOE orders. An overview of these programs is provided in Sect. 4 of the WAG 2 RI (ORNL 1990). The water sampling locations included in these programs that are relevant to the objectives of the WAG 2 RI are WOD (MS5), WOC (MS3), 7500 Bridge (GS3), WOC Headwaters (WOCHW), Melton Branch 1 (MS4), and Melton Branch 2 (MB2). These six locations are identified in Fig. 4.1.

The water quality of WOC is consistent with its use as the receiving waters of the effluents from ORNL. The major radionuclides in the stream are ${ }^{3} \mathrm{H}$ and ${ }^{90} \mathrm{Sr}$. Cesium-137 is present in the sediments and in suspension on colloids. Organics are present but typically are only evident through the analysis of sediments and fish tissue. 


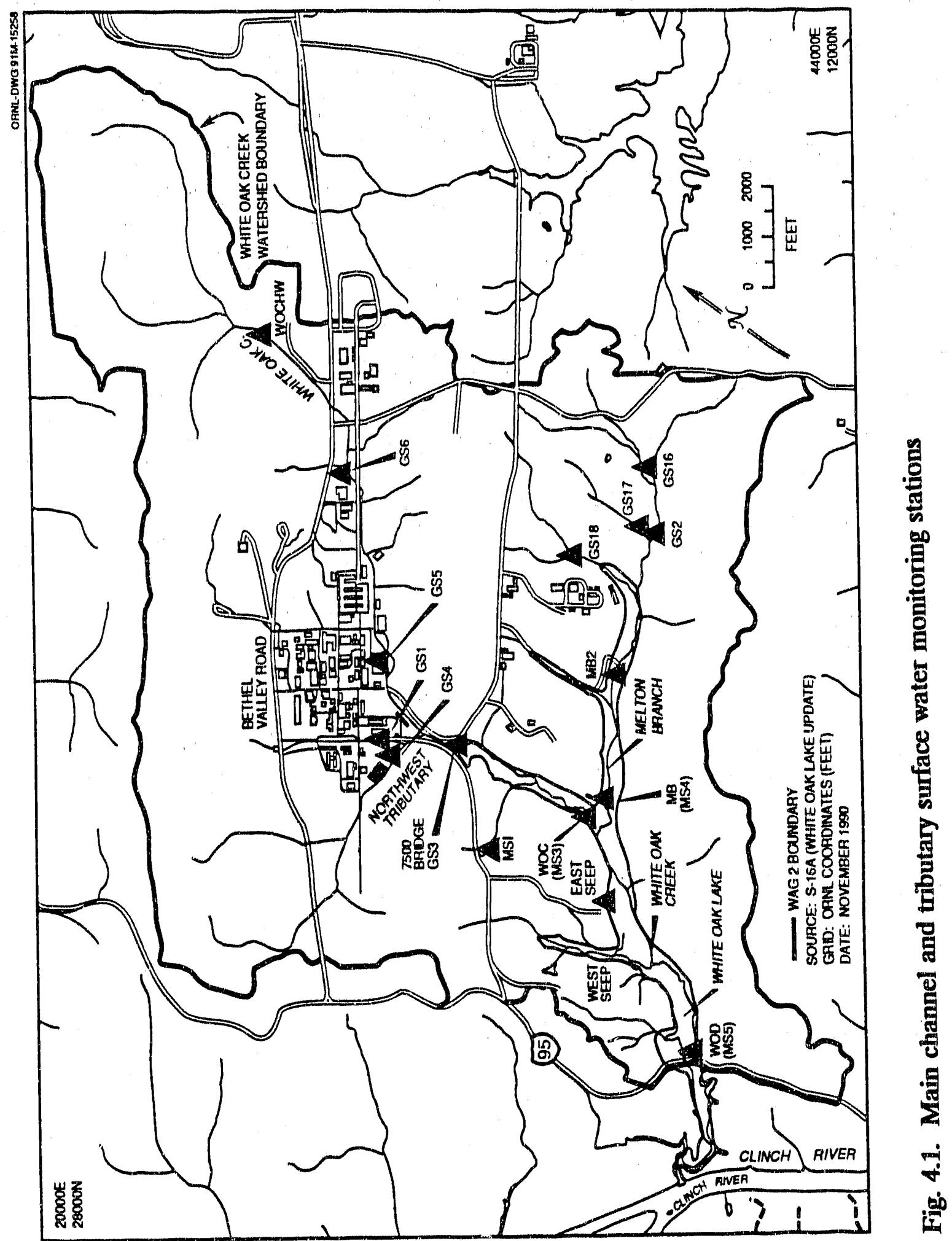




\subsubsection{Tributaries}

Tributaries to Melton Branch, WOC, and WOL are known to transport contaminants to WAG 2. A study in 1988 found that during baseflow conditions, approximately $50 \%$ of the ${ }^{3} \mathrm{H}$ and at least $10 \%$ of the ${ }^{90} \mathrm{Sr}$ mass flows (lluxes) in Melton Branch entered from the Melton Branch tributary adjacent to the eastern edges of WAGs 5 and 9 (D. S. Wickliff, ORNL, personal observation, 1988). The tributary draining WAG 4 contributes significant amounts of ${ }^{90} \mathrm{Sr}$ to WOC (Melroy and Huff 1985, Huff et al. 1982, Stueber et al. 1981). The WAG 4 tributary is also a significant source of ${ }^{3} \mathrm{H}$ to WOC. Information for ungaged streams (discharge and contaminant fluxes) is needed, along with a general improvement in existing gaging and monitoring facilities.

Contaminant fluxes in the Melton Branch tributary, Melton Branch, WOC, the WAG 4 tributary, and the WAG 6 tributaries have been found to increase as a result of rain events (Solomon et al. 1991; Huff et al. 1982). The increase of dissolved contaminant flux during storms indicates that an increase in subsurface contaminant discharge to the streams is occurring. Work by Moore (1989) and Solomon et al. (1991) suggests that the increase in groundwater discharge may be caused by shallow subsurface stormflow moving laterally above the water table. 'Therefore, ephemeral (mostly storm-driven) tributaries and seeps may be important pathways of contaminant transport to streams.

\subsubsection{Seeps}

Numerous seeps have been identified in WAG 2 or on tributaries draining inio WAG 2 [see ORNL (1990), Sect. 3.3.4.1]. Duguid (1975) reported 8 seeps in the pits and trenches area, 16 seeps in and adjacent to WAG 5, and 3 seeps in WAG 4. Radionuclide concentrations (e.g., ${ }^{90} \mathrm{Sr},{ }^{3} \mathrm{H},{ }^{137} \mathrm{Cs}$, and ${ }^{60} \mathrm{Co}$ ) were elevated in many of these seeps. Spalding and Munro (1983) found similar ${ }^{90} \mathrm{Sr}$ concentrations in some of the same seeps or seep areas in WAG 5. They also estimated that one seep on the southern perimeter of WAG 5 with an average ${ }^{90} \mathrm{Sr}$ concentration of $7.7 \mathrm{kBq} / \mathrm{L}$ made a significant contribution to the $1980{ }^{90} \mathrm{Sr}$ discharge of Melton Branch. These seeps have been identified through investigations of selected areas or by individual discovery, and there has been no recent comprehensive effort to locate seeps or to monitor contaminant fiux from seeps at the watershed level.

Seeps identified in previous studies probably represent fairly discrete areas of groundwater discharge and have been located primarily by visual inspection, with some identified following stream gravel surveys (see Sect. 3.2.5 of this report). Areas of shallow groundwater with elevated ${ }^{90} \mathrm{Sr}$ and ${ }^{3} \mathrm{H}$ concentrations have been located near Melton Branch and the WAG 4 tributary (Spalding and Munro 1983, Amano et al. 1987). Howcver, additional investigations are needed to define areas (discrete or diffuse) where contaminated groundwater is discharging directly to streams. The spacial distribution of ${ }^{90} \mathrm{Sr}$ inputs to the Northwest tributary and the WAG 4 tributary were examined by Stueber et al. (1981) and Huff et al. (1982), respectively. Stueber et al. (1981) suggested that contaminated groundwater from WAG 3 may be moving along geological strike and discharging to the Northwest tributary. A dye study showed that there was direct movement from a well in WAG 3 (42) to the Northwest tributary seep area (J. Switek, ORNL, personal communication 1991). The most prominent ${ }^{90} \mathrm{Sr}$ increase in the WAG 4 tributary occurred where a contaminated seep discharged into the channel. In 1988 sampling adjacent to WAG 5 along a stream transect indicated that approximately $44 \%$ and $30 \%$ of the tritium mass flow (flux) 
in Melton Branch was entering in two short reaches of 200 and $100 \mathrm{ft}$, respectively. At low flow, small seeps were visible in the stream channel along these reaches (D. S. Wickliff, ORNL, personal observation, 1988). Little information exists for groundwater discharge from WAG 7 and its potential for contributing to contaminant fluxes in WOC.

Recent data from the Active Sites Enviratrantal Monitoring Program indicate that two

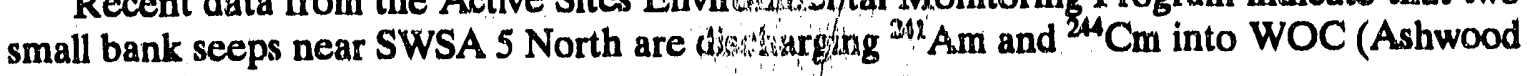
et al. 1991).

\section{OBIECTTVES AND APPROACH}

\subsection{Surface Water Sampling (Main Channels)}

Objective SW-1-Determining contaminant fluxes in surface water. The determination of contaminant flux off-site under baseflow conditions can be supported with data generated from sampling water at White Oak Dam. Components of contaminant discharge in WAG 2 can be quantified by analyzing contaminant fluxes at Melton Branch, WOC, and the 7500 Bridge. These locations correspond to the following reaches that have been identified for the risk assessment (see Preliminary Contaminant Screening for WAG 2):

- 7500 Bridge-facility input to WAG 2;

- WOC-Reach 1, upper WOC;

- Melton Branch-Reach 2, Meiton Branch; and

- White Oak Dam-Reach 3.

The current surface-water monitoring program emphasizes samples collected at baseflow. Because several important contaminants move during high-flow events, sampling will be coordinated with the group measuring sediment transport during storms (Sect. 3.2.4 of this report).

Three stream segments that are not discretely represented by the current monitoring programs are (1) WOC below the confluence with Melton Branch but above WOL (i.e., Reach 3, the area directly downgradient of WAG 7), (2) WOL distinct from the lower reach of WOC described above (i.e., the area directly downgradient of WAG 6), and (3) WOCE on the Clinch River (i.e., downstream of WOL). The CRRI project is initiating a surfacewater sampling program at the mouth of WOCE. These efforts will be most useful for characterizing conditions during storm discharge. We are working with the CRRI projert to coordinate sampling efforts to provide data for both high-discharge and baseflow conditions.

During FY 1992, data available through the compliance programs will be $u$ ed to assest the discharges and make preliminary estimates of risks associated with the major reaches that are currently sampled. These data will also be evaluated relative to the seep and tributary sampling program to determine if these sources impact the water quality of the reach sufficiently to warrant source control and to determine whether sampling of smaller reaches should be considered in future years. 


\section{4,22 Sampling and Analysix for Surface Water}

The sampling and field measurements of surface water at the major discharge monitoring stations in the FY 1992 WAG 2 sampling plan will primarily be conducted as parts of the ORNL compliance monitoring programs [see Sect. 4.1, ORNL (1990)]. The NPDES program and the Environmental Surveillance and Protection Section of the Office of Environmental and Health Protection provide an inventory of discharges into and in WAG 2 for surfacewater monitoring stations in the WOC watershed. For example, Table 4.1 describes the S\&A program at White Oak Dam.

These programs provide a comprehensive evaluation of surface water quality at baseflow conditions, except for semivolatile organic contaminants, PCBs, and pesticides. Samples for semivolatile organic contaminants $\mathrm{PCBs}$, and pesticides will be collected at least two times from each of the 6 major sampling stations during the second year of these efforts. Data for contaminant concentrations will be coupled with discharge data to construct mass balances for each major reach and identify source areas. Data from the compliance program will be coupled with data from the seep and tributary sampling efforts (described below) and the sediment sampling to identify specific contaminant inputs.

Because the compliance monitoring efforts collect samples on a set schedule, they do not systematically provide data for high flow events when sediment associated contaminants are transported. Thus, efforts to identify contaminant sources will also rely on samples collected as part of the efforts to sample contaminant fluxes during storms (Sect. 3.2.4 of this document).

The ERMA Program provide; basic information on water flow at key monitoring points within the WOC watershed to support a mass-balance approach to determining sources and sinks of contaminants and to def.ne and monitor fluxes of contaminants at the boundaries between WAGs and at surface-v/ater monitoring stations. Activities relevant to this task include upgrades in the surface-water discharge monitoring facilities to improve the hydrologic budget for the system and to identify contaminant input points.

\subsection{Tributary Sampling Program}

Efforts on tributaries will be carried out with the cooperation of the ERMA hydrologic monitoring group. The key objectives of the efforts follow.

Objectine T-1-Identify tributarics that contribute siggnificantly to contaminant flux within WAG 2 . The ability to quantify contaminant input from tributaries hinges on reliable stream discharge measurements. Weirs, flumes, and sampling stations existing on some of the tributaries need to be upgraded [see ORNL (1990), Sect. 10.4.3]. Efforts will also focus on prioritizing the need for upgrading or initiating gaging on tributaries. Ephemeral (mostly storm-driven) tributaries are not equipped for discharge measurements; however, they may be important pathways of contaminant transport to streams.

Previous studies have found that discharge-concentration relationships exist in some streams in the watershed (Solomon et al. 1991; Huff et al. 198\%). These relationships, determined by sampling the streams during a few storms, can be used to estimate yearly contaminant flux through use of the streams' discharge measurements for the year. 


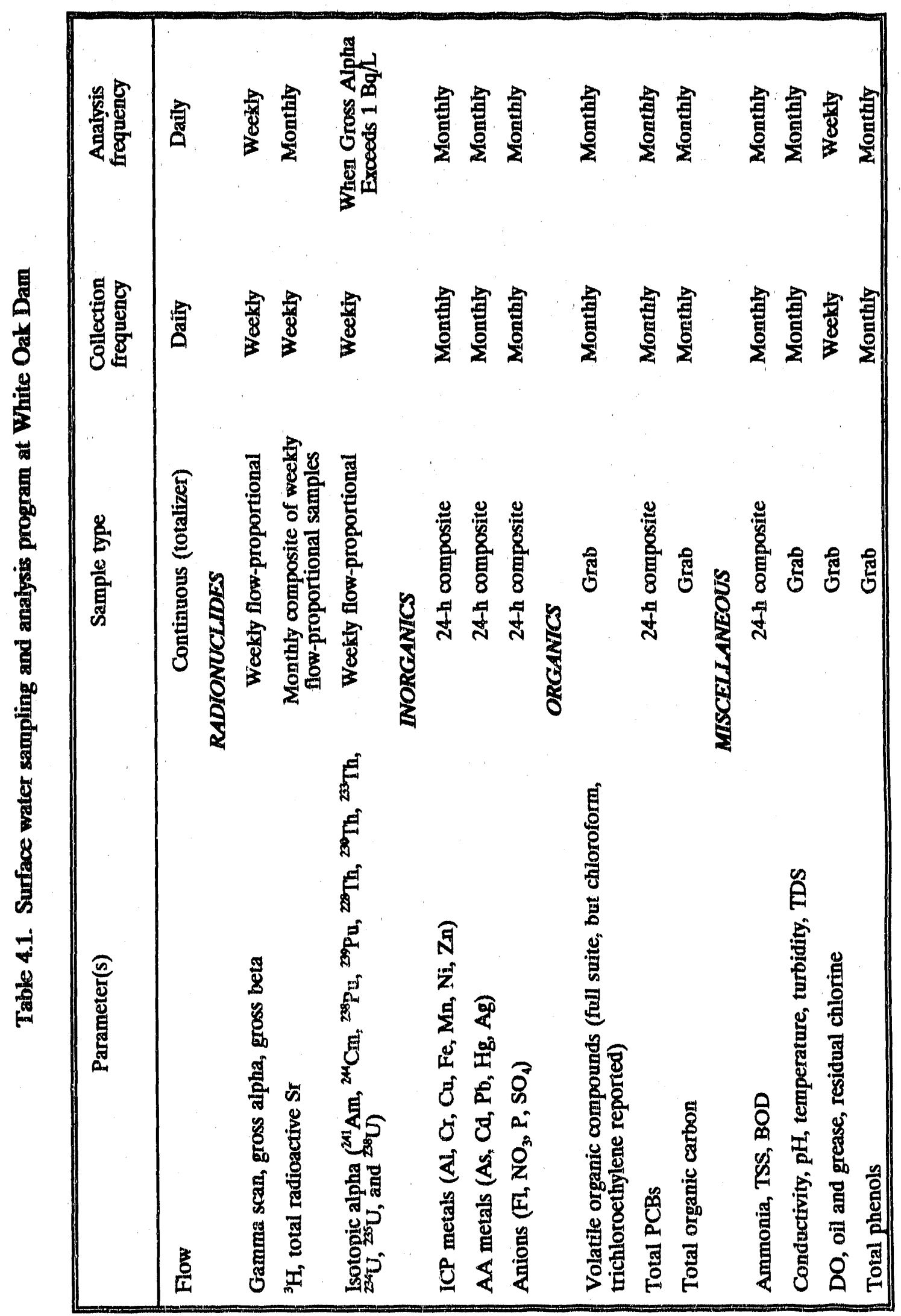


Objective T-2-Develop a tributary sampling program. The initial survey and sampling efforts (conducted during FY 1992) will be used to develop a tributary monitoring program so that key tributaries can be incorporated into a multimedia environmental monitoring of contaminant fluxes in the watershed.

The WAG 2 multimedia environmental monitoring program will address contaminant fluxes in tributaries as part of the ERMA Program (Clapp et al. 1991) with the support of the Watershed Hydrology Group (see Table 2.1). As such, this plan will include tributaries to the main branches of the WOC drainage throughout the watershed. Because most of the contaminant source areas in the watershed are adjacent to WAG 2, including areas for the upstream reaches of WAG 2 [i.e., WAG 1 and so forth (see Fig. 1.1)], supporting the ERMA Program adds relatively little work and will allow us to relate contaminant fluxes to watershed hydrology. This broader context will provide better predictive capability for temporal variation in contaminant input as well as changes in response to altered hydrologic regimes resulting from watershed modifications.

\subsection{Sampling and Analysis for Tributaries}

Tributaries will be used as spatial integrators of contaminant releases (primarily ${ }^{3} \mathrm{H}$ and ${ }^{90} \mathrm{Sr}$ ) to estimate fluxes into WAG 2 from adjacent areas. Because contaminant transport pathways and source areas may vary depending on different hydrologic conditions that exist during a year, the initial surveys will be conducted during three different conditions. Grab samples will be collected from essentially all (approximately 20) tributaries discharging into Melton Branch, WOC, and WOL (Table 4.2 and Fig. 4.1) during two baseflow conditions-one in the wet season and one in the dry season. The third sampling will be conducted just following a rainstorm. Because many small tributaries are storm driven, the third sampling may require additional sample collection. Samples will be analyzed for ${ }^{3} \mathrm{H},{ }^{90} \mathrm{Sr}$, and gamma emitting radionuclides in the dissolved load (i.e., in samples filtered through $0.45-\mu \mathrm{m}$ pore size filters) and for gamma-emitting radionuclides in the suspended load (i.e., sediment remaining on the filters). Filtered samples from tributaries with known, undetermined, or suspected alpha contamination will be analyzed for gross alpha and gross beta activity. Note that the sampling of contaminants associated with particulates will be addressed by the sediment sampling group (Sect. 3.2.4 in this plan). These efforts will be closely coordinated to identify contaminant source areas and quantify fluxes.

Contribution to contaminant flux within WAG 2 at the time of sampling can be determined from those tributaries with existing gaging stations. Flumes on the WAG 6 tributaries are being reinstrumented and upgraded to collect continuous dicsharge measurements [described in Clapp et al. (1991)]. Data from these tributaries will be used to identify the input from WAG 6, which drains directly into WOL. Contaminant concentrations from other WAG 2 tributaries without discharge measurement capabilities will be used to help prioritize the need for initiating gaging on tributaries.

The performances of weirs and sampling stations in WAG 2 are being evaluated by an Ad Hoc Committee on Weir Upgrades composed of personnel from ESD, the Environmental Surveillance and Protection Section of the Office of Environmental and Health Protection, ORNL Engineering, Project Engineering, and the ER Program. Three surface water monitoring stations in the WOC watershed are currently being reviewed for upgrade. All 
Table 4.2 Initial Scoping Sampling to Determine Contaminant Flux into and through WAG 2

\begin{tabular}{lcc}
\hline Location & $\begin{array}{c}\text { Number of Samples } \\
\text { per Campaign }\end{array}$ & \multicolumn{1}{c}{ Analyses } \\
\hline WAG 6 tributaries & 4 & ${ }^{3} \mathrm{H},{ }^{90} \mathrm{Sr}$, and gamma scan \\
West Seep (tributary) & 1 & ${ }^{3} \mathrm{H},{ }^{90} \mathrm{Sr}$, and gamma scan \\
East Seep (tributary) & 1 & ${ }^{3} \mathrm{H},{ }^{90} \mathrm{Sr}$, and gamma scan \\
$\begin{array}{l}\text { WAG } 4 \text { tributary } \\
\text { (MS1 and T2A sites) }\end{array}$ & ${ }^{3} \mathrm{H},{ }^{90} \mathrm{Sr}$, and gamma scan \\
$\begin{array}{l}\text { Northwest Tributary } \\
\text { Additional unnamed } \\
\text { tributaries }\end{array}$ & 1 & ${ }^{3} \mathrm{H},{ }^{90} \mathrm{Sr}$, and gamma scan \\
WOD & $\sim 10$ & ${ }^{3} \mathrm{H},{ }^{90} \mathrm{Sr}$, and gamma scan \\
WOC transect & 1 & ${ }^{3} \mathrm{H},{ }^{90} \mathrm{Sr}$, and gamma scan \\
MB transect including \\
MB Tributary \\
Visually located seeps
\end{tabular}


three will directly impact WAG 2 characterization. These stations are the East and West Seep drainages that are tributaries to WOC below the confluence with Melton Branch that drain WAG 7 (pits and trenches area) and the surface water monitoring station on the unnamed tributary to Melton Branch that lies in WAC2 2, near the old Homogeneous Reactor Experiment (HRE) site. Discharge and water quality data from the East and West Seep drainages are important for water budget determinations and for quantifying contaminant contributions from WAG 7. The HRE station may serve as a background water quality station to WAG 5 effluents and separate the contaminant contributions from WAG 9 as well.

The Environmental Compliance Section of the Office of Environmental and Health Protection is preparing a storm water sampling program in which, in a group of 20 to 30 key locations, mainly on tributaries and creeks in the WOC watershed, stormflow would be monitored to give a representation of the nature of ORNL non-point source storm water runoff. This strategy stresses drainage area monitoring of ER areas slated for remedial action. This effort is being coordinated with the ER Program and WAG 2 RI staff to avoid duplication of effort and to best serve the interests of both groups.

After the first year, contaminant concentration-discharge relationships will be determined for selected tributaries that are found to contribute significantly to the contaminant flux in WAG 2. A time-series of stream samples will be collected during two to three rainstorms. Rating curves that relate the instantaneous contaminant concentration to the instantaneous stream discharge will be developed. The rating curve can then be used in conjunction with continuous discharge measurements to determine contaminant flux for a period of time. Changes in these relationships may indicate changes in the source term or may help evaluate effectiveness of remedial actions. Therefore, relationships should be evaluated every few years. Data from storm sampling can also be used in model programs that separate baseflow from stormflow and their associated contaminant loads.

\subsection{Seep Sampling Program}

Objective S-1-Identify areas (discreto or diffuse) of groundwater discharge that contribute significantly to contaminant flux in the streams. The first stage of the S\&A plan will be to locate areas of groundwater discharge that bring contaminants into WAG 2. Previous studies have found that the majority of ${ }^{3} \mathrm{H}$ and ${ }^{90} \mathrm{Sr}$ enters stream reaches in discrete areas at high concentrations, causing a concentration increase in the stream. Little information is available for metals or organic contaminants. This component will focus initially on ${ }^{3} \mathrm{H}$ and ${ }^{90} \mathrm{Sr}$ and will use these data as a basis for expanded efforts to include other contaminants.

Stream discharge changes little over some stream reaches (e.g., between the major gaging stations) on WOC and Melton Branch. In addition, increases in stream discharge resulting from discrete groundwater discharges are not expected to be greater than the standard errors associated with streamflow measurements at points upstream and downstream of groundwater inputs. Consequently, significant areas of contaminated groundwater discharge to Melton Branch and WOC will be determined by measuring concentration differences in the stream transects.

When a seep is located that contributes measurably to contaminant (primarily ${ }^{3} \mathrm{H}$ and

${ }^{90} \mathrm{Sr}$ ) flux in a reach, we will attempt to determine the source of the seep based on the 
chemical signature (ionic composition) compared with known subsurface water sources (see Sect. 5 of this report). For seeps that are found to be significant contributors to contaminant fluxes, we will

1. identify the sources of these inputs (contaminant source area and subsurface pathway),

2. investigate the relationship between watershed hydrology and contaminant input,

3. incorporate selected seeps or areas of groundwater discharge into a multimedia environmental monitoring of contaminant fluxes in the watershed,

4. evaluate the contribution of individual inputs to human health and environmental risk,

5. conduct preliminary evaluation of the need for intexim corrective measures for individual seeps or areas of cuntaminated groundwater discharge, and

6. inform those responsible for characterization of the WAG to ensure that the information becomes part of the record for that area for follow-up work.

The initial survey and sampling efforts (conducted during FY 1992) will be used to develop a seep monitoring program. Key seeps and discharge areas will be monitored to provide information useful for extrapolating to the other seeps of interest in the watershed.

The WAG 5 RI project is also interested in seeps that carry contaminants from WAG 5 into Melton Branch and WOC in WAG 2. Similarly, the WAG 1 RFI project is interested in seeps in WAG 1 that discharge into WOC above WAG 2. We are coordinating with those projects to share the sampling efforts and data. Most imporiant, however, we need to be sure that we employ comparable approaches so that data gathered by a project can be used elsewhere in the watershed. We have met with representatives of the WAG 5 RI and WAG 1 RFI and will be cooperating on these efforts by developing standard procedures and sharing data collection responsibilities. Our goal is to cooperate with these programs to provide better information and to develop a better understanding of contaminant pathways and fluxes for the ORNL WAGs.

\subsubsection{Sampling and Analysis for Seeps}

The initial survey for groundwater discharge areas in WAG 2 will be conducted in conjunction with the tributary sampling during the three different hydrologic conditions. Samples will be collected from WOC and Melton Branch approximately every $100 \mathrm{~m}$ and analyzed for ${ }^{3} \mathrm{H}$ and dissolved ${ }^{90} \mathrm{Sr}$ activity. Incremental increases in concentrations between sampling locations on the stream reaches will identify areas of contaminated groundwater discharge. Specific conductance and temperature of the stream water will also be measured during the first sampling to see if changes or anomalies occur and correlate with changes in radionuclide concentrations (areas of contaminated groundwarer discharge). More intensive sampling will be done within $100 \mathrm{~m}$ intervals where substantial contaminant inputs are identified.

Areas of groundwater discharge will also be located by visual inspection during the initial survey. Grab samples will be collected from seeps (approximately 20) in WAG 2 located by previous investigations and by the initial walkover. In addition to ${ }^{3} \mathrm{H}$ and ${ }^{90} \mathrm{Sr}$, samples will be analyzed for gamma-emitting radionuclides. Because many seeps are storm-driven, the third sampling will be conducted following a major rainstorm that is observed to activate ephemeral groundwater discharge areas. As noted previously, we will initially emphasize 
radiological contaminants to identify input areas and to provide a basis for the design of later, more comprehensive efforts.

Idfsally, we could calculate the contaminant mass flux via individual seeps by comparing the stream discharge $(Q)$ and contaminant concentration $(C)$ upstream and downstream of discrete inputs (i.e., $Q \times C$ dowistream $=Q \times C$ upstream $+Q \times C$ seep; and $Q \times C$ seep $=$ contaminant mass flux). Existing evidence suggests that the individual seeps contribute measurably to contaminant concentration in the stream water but do not increase flow by quantitles measurable by field equipment. Thus, it will seldom be possible to measure changes in $Q$ upstream and downstream of $t i$ seep input. Discharge can conservatively be estimated to change very little, and mass input can be estimated by the change in concentration of the contuminant in stream water upstream and downstream of discrete inputs.

Unless we are able to identify' unique constituents of a seep (e.g., high concentration of an ion-see Sect. 5.2.3 of this plan), we will use changes in contaminant concentrations to identify inputs and base mass fluxes on changes in concentrations in the stream water, assuming changes in discharge are minor. We recognize that this limits our ability to identify inputs that do not contribute at least $10 \%$ to the ${ }^{3} \mathrm{H}$ or ${ }^{90} \mathrm{Sr}$ mass fluw. (The $10 \%$ figure reflects the precision of analyses and sampling variability.) However, this approach will allow us to identify the major contributors to contaminant flux.

Following the preliminary surveys to locate groundwater discharge areas, the approach will be to monitor 8 to 12 stream transects, seeps, or storm-driven tributaries that are suspected to be major perennial contributors to contaminant flux. Of these, four to six will be chosen for intensive monitoring, specifically addressing contaminant input related to hydrologic conditions. For groundwater discharging directly into the stream channel, we will install small piezometer wells near the stream bank to collect groundwater and sample the stream transect for changes in contaminant concentrations. Samples collected from these seeps will be analyzed for metals and major ions to determine the ionic composition of the water, which will aid in identifying its source. Metals and major ions will be determined several times annually. During the first 2 years of this program, we will not analyze seep samples for organic contaminants unless stream sediment sampling suggests a major source of organic contamination within a reach. Samples from selected seeps may be analyzed for transuranics if data from sediment sampling or data from other programs suggests a specific seep may be a pathway for transuranics.

Flow rates from some seeps and small tributaries may be determined volumetrically at discrete points in time. Methods for measuring continuous discharge will be investigated. Data from intensively monitored seeps may be used to estimate annual flux from other seeps in the watershed.

\subsection{METHODS}

\subsubsection{Surface Water}

Methods for surface water analyses for data generated by the compliance monitoring programs are presented in Table 4.3. 
Tablo 4.3. Methoda for surface water analysis

\begin{tabular}{|c|c|c|}
\hline Analysis & Method" & Condition ${ }^{b}$ \\
\hline Gamma scan & 901.1 & $\mathbf{U}, \mathbf{A}$ \\
\hline Gross alpha and beta & 900.0 & $\mathrm{U}, \mathbf{A}$ \\
\hline Tritium & 906.0 & $\mathbf{U}$ \\
\hline Total strontium-90 & 905.0 & $\mathbf{U}, \mathbf{A}$ \\
\hline Isotopic alpha & AC-MM-2-0972 & $\mathrm{U}, \mathrm{A}$ \\
\hline ICP metals & 200.7 & $\mathrm{U}, \mathrm{A}$ \\
\hline $\begin{array}{c}\text { AA metals } \\
\mathrm{As} \\
\mathrm{Cd} \\
\mathrm{Pb} \\
\mathrm{Hg} \\
\mathrm{Ag}\end{array}$ & $\begin{array}{l}206.2 \\
213.2 \\
239.2 \\
245.1 \\
242.1\end{array}$ & $\begin{array}{c}\mathbf{U}, \mathbf{A} \\
\mathbf{U}, \mathbf{A} \\
\mathbf{U}, \mathbf{A} \\
\mathbf{U}, \mathbf{A} \\
\mathbf{U}, \mathbf{A}\end{array}$ \\
\hline Anions & 429.0 & U \\
\hline Volatile organics & 624.0 & $\mathbf{U}$ \\
\hline Total PCBs & 608.0 & U \\
\hline Total organic carbon & 415.1 & $\mathbf{U}$ \\
\hline Ammonia & 350.3 & $U$ \\
\hline TSS & 160.2 & $\mathbf{U}$ \\
\hline $\mathrm{BOD}$ & 507.0 & $\mathbf{U}$ \\
\hline TDS & 160.1 & $\mathbf{U}$ \\
\hline Oil and grease & $503 A$ & $\mathbf{U}$ \\
\hline Total phenos & 420.1 & U \\
\hline
\end{tabular}

"EPA Clean Water Act.

${ }^{b} U=$ unfiltered, $A=$ acidified.

'ORNL Analytical Chemistry Division procedure. 


\subsubsection{Tributaries and Seepe}

Samples from the FY 1992 sampling of tributaries, WOC and Melton Branch reaches, and seeps will be sampled following procedures by Kimbrough et al. (1990) for grab sample collection. Samples will be analyzed for ${ }^{3} \mathrm{H}$, ${ }^{91} \mathrm{Sr}$, gamma-emitting radionuclides by the ESD (Table 4.4). A procedure adapted from EPA Method 906.0 for ${ }^{3} \mathrm{H}$ analyses in drinking water (EPA 1980c) will be used. Samples for ${ }^{90} \mathrm{Sr}$, gross alpha, gross beta, and gamma scans will be filtered and acidified ( $\mathrm{pH}<2$ ). Cerenkov radiation counting (Ross 1969) will be used to determine ${ }^{90} \mathrm{Sr}$ activity. At least one in 20 samples for ${ }^{90} \mathrm{Sr}$ will be analyzed by EPA Method 905.0 as a quality check. All methods will be reviewed by the ERD-APO. Gross alpha and beta analyses wiil be completed by the ORNL Analytical Chemistry Division (EPA Method 900.0). Results from the initial scoping study will be used to develop tributary and seep monitoring programs and more complete S\&A procedures. Samples for metal analysis and ionic composition will be analyzed by the ORNL Analytical Chemistry Division.

\subsection{QUALTYY ASSURANCE}

Data for monitoring stations on the main channels are provided by the NPDES and the Environmental Surveillance monitoring programs. These programs, described below, meet data quality objectives (DQOs) for the WAG 2 S\&A Plan.

Main Channels. The QA programs for compliance with the NPDES permit and the DOE Environmental Orders will ensure the quality of the surface water data collected and used in the first year of the WAG 2 RI. Compliance sampling and field measurements are controlled by the SOP of the Environmental Surveillance and Protection Section of the Office of Environmental and Health Protection. These procedures include requirements for sampling methods, chain of custody, and documentation. They are based on guidance from EPA and meet or exceed EPA protocol requirements.

Discharge Measurement. The USGS maintains eight of the surface-water discharge monitoring stations. The USGS Water Resources Division is a national program recognized for its expertise and experience in collecting discharge data of the highest quality and accuracy. All additional sites (discharge and precipitation) are included under a program that is conducted in accordance with the QA program of the ESD (Roberson and Logsdon 1989), which is consistent with ANSI/NQA-1. A separate quality assurance plan has been prepared and is updated as necessary. All monitoring sites will be maintained, and instrumentation will be serviced routinely.

Soep and Tributaries. Procedures for sampling methods of the scoping study of tributaries, WOC and Melton Branch reaches, and seeps will be developed and documented and reviewed by the ERD-APO. Duplicate samples for QC purposes will be collected at a rate of about one per ten samples. Laboratory-prepared standards and blanks will be counted with each group of samples being analyzed for ${ }^{3} \mathrm{H}$ and ${ }^{90} \mathrm{Sr}$. In addition, EPA QA/QC samples will be analyzed by the proposed methods. 
Table 4.4. Methods for initial scoping study of tributaries and coeps

\begin{tabular}{|c|c|c|c|}
\hline Analysis & Method & Laboratory & Condition \\
\hline Tritium & EPA - 906.0 & ESD & $\mathrm{U}$ \\
\hline Strontium-90 & $\begin{array}{l}\text { Cerenkov radiation } \\
\text { counting }\end{array}$ & ESD & $\mathbf{F}, \mathbf{A}$ \\
\hline Gamma scans & $\begin{array}{l}\text { Development of } \\
\text { procedures is in } \\
\text { progress }^{c}\end{array}$ & ESD & $\mathbf{F}, \mathbf{A}$ \\
\hline Gross alpha & EPA - 900.0 & $\mathrm{ACD}^{b}$ & $\mathbf{F}, \mathbf{A}$ \\
\hline Gross beta & EPA - 900.0 & $\mathrm{ACD}$ & $\mathbf{F}, \mathbf{A}$ \\
\hline Metals & $\begin{array}{l}\text { ICP } \\
\text { AA for potassium } \\
\text { EPA - } 258.1\end{array}$ & $\begin{array}{l}A C D \\
A C D\end{array}$ & $\mathbf{F}, \mathbf{A}$ \\
\hline
\end{tabular}

- Analytical services will be coordinated through the ERD-APO.

- $\mathbf{U}=$ unfiltered, $\mathbf{F}=$ filtered, $\mathbf{A}=$ acidified.

- Analytical procedure presented in Appendix C. 


\title{
5. GROUNDWATER SAMPLING PLAN
}

\author{
R. H. Ketelle, G. K. Moore, and M. S. Tardiff
}

\subsection{INTRODUCTION}

WAG 2 includes primarily the WOC and Melton Branch floodplains and toe slopes from adjacent upland areas. Because of the relatively low topographic setting of WAG 2, the area is a receptor and conduit for overland flow, stormflow, and groundwater inflows from adjoining areas. Overland flows and stormflows are perceived to move relatively quickly through WAG 2, with the potential for contaminants in these flows to adsorb to soils or sediment within WAG 2 or to enter the groundwater reservoir. The groundwater reservoir in WAG 2 includes saturated alluvium beneath and adjacent to WOC and Meiton Branch, as well as a thin water table aquiler in saprolite cornected to water-filled fractures in the underlying bedrock. Rates of groundwater flow and contaminant movement through the groundwater reservoir are slower than those observed in either surface flows or stormflows. The groundwater reservoir provides the continuous source of water that sustains baseflows in local streams. No solid waste disposal activities are known to have occurred within WAG 2; however, contamination of soils, sediments, and groundwater has occurred as a result of past discharges of contaminated liquid effluent from ORNL, from leaks of liquid waste transfer lines, and from inflow of groundwater from adjacent WAGs. Because groundwater discharges to surface water prior to leaving the watershed, the linking of groundwater and surface water activities is essential. The groundwater component will focus on the evaluation of existing information and data now becoming available from ORNL monitoring programs. This component will be linked with ERMA groundwater efforts and other activities under way in the WOC watershed.

\subsubsection{Review of Existing Dala}

Several hundred wells in the WOC watershed are potential sources of information for the WAG 2 effort. Data available from S\&A of well waters within WAG 2 and from wells constructed in adjacent WAGs on the boundary of WAG 2 indicate that radionuclide contamination of groundwater is serious but not widespread. Less evidence exists regarding groundwater contamination with organic compounds and metals; however, the available data do not provide sufficient resclution of water quality patterns to accurately define contamin\&nt distributions within WAG 2. A summary review of contaminant detection in existing wells in WAG 2 is presented in Sect. 6.3 of the WAG 2 RI plan (ORNL 1990).

\subsection{Objectives}

Long-range objectives of the WAG 2 groundwater sampling plan are to document the distribution of groundwater contaminants within the WAG, understand mechanisms that control contaminant movement through the groundwater pathway in WAG 2, and to provide data and parameter estimates for use in remedial alternative design and evaluation. Because WAG 2 is a receptor and pathway for contaminant fluxes from adjacent. WAGs, it is imperative that remedial investigations and remedial actions here draw on information from the adjacent areas and that the WAG 2 evaluation be sensitive to effects of as yet unseen contaminants migrating from adjacent WAGs. For these reasons the WAG 2 investigation 
will progress for the duration of remedial investigations at the neighboring WAGs and will provide a synthesis of information on contaminant fluxes leaving the watershed via WOC.

The first objective is to construct a data set that will include information for geologic formation, monitored zone, hydraulic conductivity, water level, and other parameters to serve as a reference for hypothesis testing and groundwater flow and geochemical data interpretation.

The second objective is to evaluate data for groundwater geochemistry to develop a picture of the nature and extent of contaminant transport in groundwater. Statistical pattein recognition analysis will be used to evaluate the data.

The third objective is to conduct a series of hydrogeologic investigations conducted in conjunction with and supported by the ERMA program (Clapp et al. 1991) to evaluate the role of groundwater flow in the migration of contaminants from adjacent WAGs through WAG 2 to streams. These efforts focus on measurements of water levels and physicochemical data for selected wells, the identification of discrete fiow zones, evaluation of contaminant distributions relative to hydraulic gradients, and coordination with the seep sampling efforts to evaluate groundwater flow paths.

\subsubsection{Approach}

Achievemen: of the stated objectives rf the WAG 2 RI requires thorough review and interpretation of existing data, further investigation of areas showing anomalous water quality or hydrogeologic character, and long-term monitoring of the hydrologic system within and around WAG 2. During FY 1992, planned WAG 2 RI activities include review and interpretation of existing water quality and hydrogeologic data, deployment of field instrumentation to record variation of hydrologic and physicochemical parameters at selected wells, continuation of S\&A of groundwater at WAG 2 perimeter monitoring wells, and geophysical investigation in the vicinity of the groundwater chloride anomaly on the WOC floodplain.

\subsection{WATIER COLLECTION, FIEID MEASUREMENTS, AND SOUR.CE IDENTIFICATION}

\subsubsection{Well Sampling and Water Quality Analyses}

During FY 1992, ORNL WAG perimeter water quality monitoring wells will be sampled semiannually by the ORNL Environmental Surveillance program (Greene 1991). Analyses that will be performed on samples from WAG 2 perimeter wells are listed in Table 5.1. The locations of WAG 2 perimeter monitoring wells are shown on Fig. 5.1.

Pending resulis of the review of existing data from monitoring wells and piezometers, additional confirmatory sampling of selected piezometers within or adjacent to WAG 2 may be performed. Analytes to be measured on such samples will be determined on a case by case basis. Analytical procedures and QA measures for these samples will be consistent with those used for the WAG 2 perimeter well monitoring program. 
Table 5.1 ORNL WAG Perimeter Well Analyses

\begin{tabular}{|c|c|c|c|c|c|c|}
\hline $\begin{array}{l}\text { Order of } \\
\text { Collection }\end{array}$ & Analysis & Method & Laboratory & $\begin{array}{c}\mathbf{U}=\text { Unfiltered } \\
\mathbf{F}=\text { Filtered }\end{array}$ & $\begin{array}{l}\text { RDL } \\
\mu \mathrm{g} / \mathrm{L}\end{array}$ & $\begin{array}{l}\text { Reporting } \\
\text { Units }\end{array}$ \\
\hline 1,2 & Volatiles $\times 2$ & RCRA-8240 & QAL & $U$ & - & $\mu \mathrm{g} / \mathrm{L}$ \\
\hline 3 & Semi-volatiles & RCRA-8270 & QAL & $\mathrm{U}$ & - & $\mu \mathrm{g} / \mathrm{L}$ \\
\hline $4,5,6,7$ & TOX $\times 4$ & RCRA-9020 & CPA & U & 5 & $\mu \mathrm{g} / \mathrm{L}$ \\
\hline $8,9,10,11$ & $\operatorname{TOX} \times 4$ & RCRA-9060 & CPA & $\mathrm{U}$ & 1000 & $\mathrm{mg} / \mathrm{L}$ \\
\hline 12,13 & $\begin{array}{l}\text { Metals, ICPb } \\
\text { (AA) Potassium }\end{array}$ & $\begin{array}{l}\text { RCRA-6010 } \\
\text { RCRA-7610 }\end{array}$ & $\mathrm{CPA}$ & $\mathrm{U}, \mathrm{F}$ & & $\begin{array}{l}\mathrm{mg} / \mathrm{L} \\
\mathrm{mg} / \mathrm{L}\end{array}$ \\
\hline 14,15 & $\begin{array}{l}\text { Metals, ICP/MS } \\
\text { (CVAA) Mercury }\end{array}$ & $\begin{array}{l}\text { RCRA-6020 } \\
\text { RCRA-7470 }\end{array}$ & $\mathrm{CPA}$ & $U, F$ & 0.2 & $\begin{array}{l}\mathrm{mg} / \mathrm{l} \\
\mathrm{mg} / \mathrm{L}\end{array}$ \\
\hline 24,25 & $\begin{array}{c}\text { Rad } \\
\text { Total Strontium } \\
\text { Gamma Spec } \\
\left({ }^{80} \mathrm{Co},{ }^{17} \mathrm{Cs}\right) \\
\text { Gross Alpha } \\
\text { Gross Beta }\end{array}$ & $\begin{array}{l}\text { CAWW-905.1 } \\
\text { CAWW-901.1 } \\
\text { CAWW-900.0 } \\
\text { CAWW-900.0 }\end{array}$ & LLL & $\mathrm{U}, \mathrm{F}$ & $\begin{array}{c}0.12 \\
- \\
0.30 \\
0.62\end{array}$ & $\mathrm{~Bq} / \mathrm{L}$ \\
\hline 22,23 & Tritium & CAWW-906.0 & LLL & $U, F$ & 200 & $\mathrm{~Bq} / \mathrm{L}$ \\
\hline 16 & Anions & CAWW-300.0 & CPA & U & - & $\mathrm{mg} / \mathrm{L}$ \\
\hline 17 & Total phenolics & RCRA.9065 & $\mathrm{CPA}$ & $\mathrm{U}$ & - & $\mathrm{mg} / \mathrm{L}$ \\
\hline 18 & TSS & CAWW-160.2 & CPA & $\mathrm{U}$ & - & $\mathrm{mg} / \mathrm{L}$ \\
\hline 19 & $\mathrm{TDS}$ & CAWW-160.1 & $\mathrm{CPA}$ & $\mathrm{F}$ & - & $\mathrm{mg} / \mathrm{L}$ \\
\hline 20,21 & Alkalinity & CAWW-310.1 & $\mathrm{CPA}$ & $\mathrm{U}, \mathrm{F}$ & - & $\mathrm{mg} / \mathrm{L}$ \\
\hline $7,12,18,25$ & Temperature $\times 4$ & 170.1 & Field & $U$ & - & ${ }^{\circ} \mathrm{C}$ \\
\hline $7,12,18,25$ & $\mathrm{pH} \times 4$ & 150.1 & Field & U & 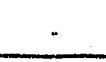 & $\mathrm{pH}$ units \\
\hline $7,12.18,25$ & Conductivity $\times 4$ & 120.1 & Field & $\mathrm{U}$ & - & $\mathrm{mS} / \mathrm{cm}$ \\
\hline 25 & Redox & $\begin{array}{l}\text { ESP.SOP- } \\
003.023\end{array}$ & Field & $U$ & . & $\mathrm{mV}$ \\
\hline 25 & DO & 360.1 & Field & $\mathrm{U}$ & - & $\mathrm{ppm}$ \\
\hline
\end{tabular}

-Volatiles - Analyze one sample only (Analyze a second sample if timits are exceeded on first sample).

'Al, Sb, As, Ba, Be, B, Cd, Ca, Cr, Co, Cu, Fe, Pb, Mg, Mn, Mo, Ni, Se, Si, Ag, Na, V, Zn

${ }^{\circ} \mathrm{Ag}, \mathrm{As}, \mathrm{Pb}, \mathrm{Sb}, \mathrm{Se}, \mathrm{Ti}$ 


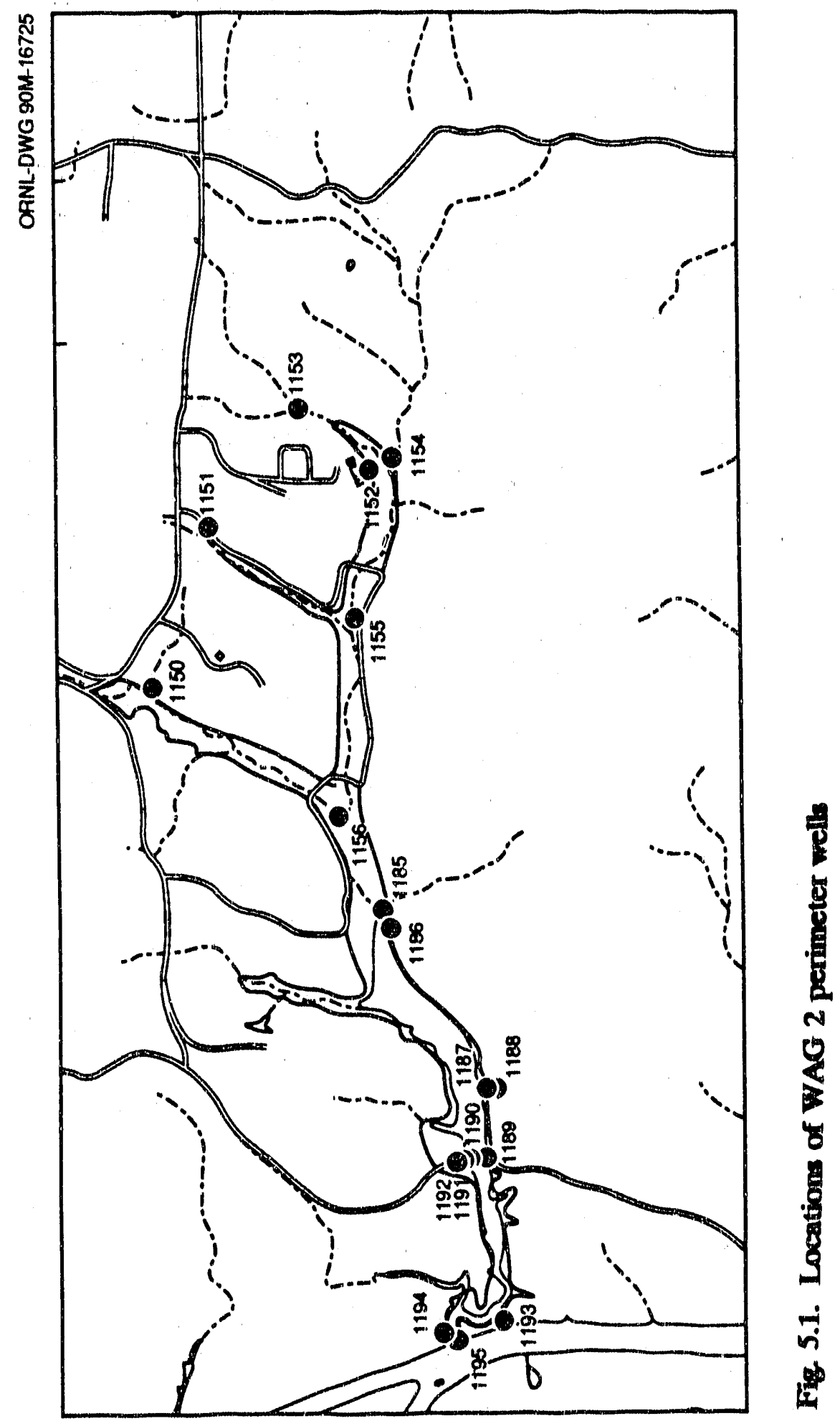




\section{WAG 2 Geology, Well Construction, and Water Level Data Interpretation}

To create a coherent reference frame for hypothesis testing and groundwater flow and geochemical data interpretation, a data set for WAG 2 and adjacent areas will be created including definition of geologic formation, monitored zone, hydraulic conductivity, water level, and other parameters. The data set will include all wells from which geochemical data are obtained and other wells and borings that help define physical system boundaries and conditions. A query of the ORNL consolidated data base for wells indicates that in the area including WAG 2 and a $300 \mathrm{ft}$ wide buffer strip surrounding the WAG there are 534 existing wells (251 pre-RAP piezometers, 82 water quality monitoring wells, 111 RAP piezometers, 15 HHMS wells, 57 hydrofracture-related wells, 8 impoundment wells, 4 TARA wells, and 6 USGS wells). Data from these wells will be used to construct diagrams showing the relationships of physical boundaries (e.g., soil/bedrock interface, water table, and stratigraphic boundaries), which is useful for display and interpretation of groundwater flow systems and geochemical zonation. This task is interactive with the evaluation of groundwater geochemistry and will be performed in conjunction with the ERMA groundwater program (Clapp et al. 1991).

\subsection{Evaluation of Data for Groundwater Geochemistry}

To evaluate groundwater quality and develop a picture of the nature and extent of contaminant transport in the groundwater, it is necessary to have data for background water quality and to be able to determine the contribution of water from different areas to a sample collected from a well. If the chemical :omposition of contaminated water and background uncontaminated water can be determir. $d$, then the relative contribution of each water type to the composition of a water sample from a specific well can be determined and used to follow the course of contaminant flow. Given the heterogeneous nature of geologic formations in Melton Valley, there may be several distinct types of background water quality, each one highly dependent on the geology immediately surrounding the screened interval in a particular well. Thus, recognition of background water quality will entail an analysis of a broad range of geochemical parameters (see Table 5.1) conducted in the context of detailed descriptions of the screened interval geology. Further fine tuning of the geochemical interpretations may require incorporation of other hydrogeological data to characterize the flow path/production zone (e.g., transmissivity and hydraulic conductivity).

The ORNL ESP section monitors groundwater quality as part of several programs coordinated by the ORNL groundwater coordinator. A network of 178 RCRA-quality groundwater wells has been installed on the perimeters of the ORNL WAGs (Fig 5.1). Beginning in 1991, they are being sampled semiannually for metals, radionuclides, organics, and other parameters as part of a Remedial Action Monitoring Program (Greene 1991). These data, combined with data sets of comparable quality from older wells in the vicinity, provide a data base amenable to several forms of factor analysis that serve to identify patterns in large data sets. Once factors have been identified, interpretation of the factors begins an iterative process of refining and incorporating knowledge about the formation geology and hydrogeology. Through the process of performing the review and interpreting existing data,

iterative hypothesis testing of hydrogeologic concepts and geochemical concepts that contribute to the overall conceptual model of groundwater movement will be used to strengthen and quantify the understanding of WAG 2 hydrology. 
The objectives of this activity are

Objective 1: Review the existing and incoming data for groundwater quality for the WOC watershed with emphasis on WAG 2. The focus of the review will be on interpretations of groundwater quality as it relates to identification of contaminant sources and contaminant trans ${ }_{k}^{2}$ ort processes.

Objective 2: Conduct statistical pattern recognition analysis of WOC watershed groundwater quality data to (1) identify background wells, (2) identify background or baseline groundwater quality as a function of the screened interval (i.e., geologic formation, regolith vs bedrock), (3) identify contaminant concentrations above background, and (4) identify groundwater contaminant sources.

The results of the initial evaluation will be used to develop questions concerning contaminant sources, identify data needed to address those questions, and design a groundwater sampling plan to obtain the data required.

\subsection{Hydrogeologic Investigations}

Hydrogeologic investigations for WAG 2 will focus on developing an understanding of the importance of groundwater flow in migration of contaminants from adjacent WAGs through WAG 2 to discharge at the local streams. Tasks identified include

- instrumentation of selected wells to document water level and physicochemical changes that may occur in response to seasonality and major precipitation events,

- interpretation of WAG 2 borehole flowmeter data to identify discreet flow zones in wells for the purpose of determining thickness and transmissivity variability areally and vertically,

- interpretation of contaminant distribution from existing data in consideration of area hydraulic gradients,

- interaction with the seep study team to cooperatively analyze the groundwater flow paths leading to seeps (see Sect. 4.2 .6 of this plan).

These investigations rely partly on efforts supported by the ERMA program.

\subsubsection{Stormflow Zone and Matris Diffusion}

The stormflow zone is a shallow zone, corresponding to the root zone of the vegetation, that is much more permeable that the unsaturated zone. Stormflow is transient but may be an important pathway for water after precipitation events. The role of the stormflow zone for contaminant transport into WAG 2 needs to be evaluated. An understanding of hydrologic and contaminant fluxes in the stormflow zone and techniques to monitor stormflow zone are needed to ensure that the remedial alternatives selected are appropriate for mitigating contaminant fluxes. 
The efforts are directed at determining (1) the importance of the stormflow zone as a pathwey of contaminant transport and (2) whether the diffusion of contaminants into the soil and rock matrices results in the creation of secondary source areas that can continue to release contaminants aftur contaminant releases for primary sources (waste trenches) have been eliminated. These efforts are being conducted primarily as part of the special investiggations component of ERMA and are being partly supported by the WAG 2 RI project. Efforts for ERMA include defining the tritium plume downgradient of trenches in WAG 5 , conducting the stormflow zone monitoring described below, and intensively monitoring the streams during storms. The DOE OHER is supporting studies of subsurface transport and matrix diffusion in a subsurface weir facility in Melton Valley several hundred meters upgradient of the borders of WAG 2. The subsurface facility is located at the bottom of a hillslope equipped with an elaborate array of tensionmeters, multipore region soil water samplers, and subsurface weirs automated to quantify three-dimensional hydrologic and chemical fluxes (Jardine et al. 1990). This group is also using undisturbed soil columns to study the transport of solutes (including strontium and cobalt) to provide information for modeling in situ subsurface contaminant transport (Jardine and Jacobs 1991). The information on water and solute movement through soils, and on the processes that influence it, will provide important basic information for constructing a conceptual model of contaminant movement in WAG 2.

FY 1992 studies of stormflow zone transpurt will be conducted near the boundary of WAG 5 and WAG 2. Water levels will be continuously monitored in wells and in stormflow tubes at depths of 20,50 , and $80 \mathrm{~cm}$. Groundwater samples will be analyzed to determine the lateral and vertical extent of tritium contamination. Tritium was chosen because available information suggests that tritium is the primary contaminant being released from WAG 5.

Monthly samples for the analysis of tritium will also be collected from two drive-point wells and from a seep in a the Melton Branch tributary to the east of WAG 5. These data will be used to determine the importance of the stormflow zone for contaminant transport, whether contaminant concentrations are increasing or decreasing in discharges from the waste trenches, and whether primary sources (waste trenches) or secondary sources (soil and rock matrices) are more important for future releases of tritium. These data will have implications for the sources of other contaminants as well.

The need for additional sampling of water and contaminant fluxes in the stormflow zone near WAG 2 will be determined by an evaluation of the FY 1992 data. Such efforts may include continuous water-level monitoring and analyses of water samples from the stormflow zones below WAGs 4 through 8 . However, the stormflow zone may prove not wo be an important pathway for contaminant transport, and the existing network of water quality monitoring wells may prove adequate for an estimation of the contaminant flux at the WAG 2 boundary. 


\title{
6. ECOLOGICAL ASSESSMENT
}

\author{
T. L. Ashwood and G. W. Suter
}

\subsection{INTRODUCTION}

\subsubsection{Overview}

Understanding the movement of contaminants through the ecosystem is important for three reasons. First, the biota may be at risk if contaminant levels are high enough to affect population parameters such as fecundity. Second, some species (e.g., deer, waterfowl, wild turkeys, many fish) are food sources for humans and may provide a pathway that is important in a human health risk assessment. Finaliy, some species that feed at the top of the food chain may serve as ecological indicators of contamination within the WAG 2 ecosystem. Ecological indicator species may be useful as long-term monitors of changes in contaminant levels or contaminant availability in the system. That is, some species can provide information on the ecologically relevant levels of contamination within a system that is not available from other measurements.

Because WAG 2 serves as an integrator of contamination from source WAGs, it is essential that sampling and analysis of all compartments in WAG 2 be conducted to identify changes in contaminant inputs over time. Sections 3 through 5 of this plan address the conceptual models and long-term monitoring of soils, sediments, groundwater, and surface water. This section presents the conceptual framework and specific activities associated with long-term monitoring of the biota in WAG 2.

The ecological assessment plan is based on close coordination of three sources of biological data at ORNL: (1) the ORNL BMAP, (2) biological sampling conducted to support remedial investigations in specific source WAGs (e.g., WAG 5), and (3) biological monitoring within WAG 2 (Fig. 6.1). Such coordination is enhanced by involvement of the same key individuals in the planning and implementation of all three programs.

It is neither cost effective nor essential to develop an extensive monitoring program covering the wide array of species within WAG 2. Extensive data on the radiological and nonradiological contaminants in aquatic biota already exist for WAG 2 (see ORNL 1991 and Blaylock et al. 1991b). As already mentioned, close co rrdination with the BMAP and other WAG RIs will provide a means to augment existing data and develop the long-term monitoring program needed for WAG 2. Finally, we already understand the fundamental ecological processes that control transport of contaminants through the WAG 2 ecosystem; we are thus able to focus our investigations on those few critical areas (primarily associated with nonradiological contaminants in the terrestrial system) where filling data gaps will enable us to predict and monitor the effects of changes in contaminant inputs.

\subsubsection{Assessment Endpoints}

The first step in conducting an ecological assessment is to identify assessment and measurement endpoints (Warren-Hicks et al. 1989). Assessment endpoints should be socially and biologically relevant, be operationally definable and measurable, be susceptible to the 

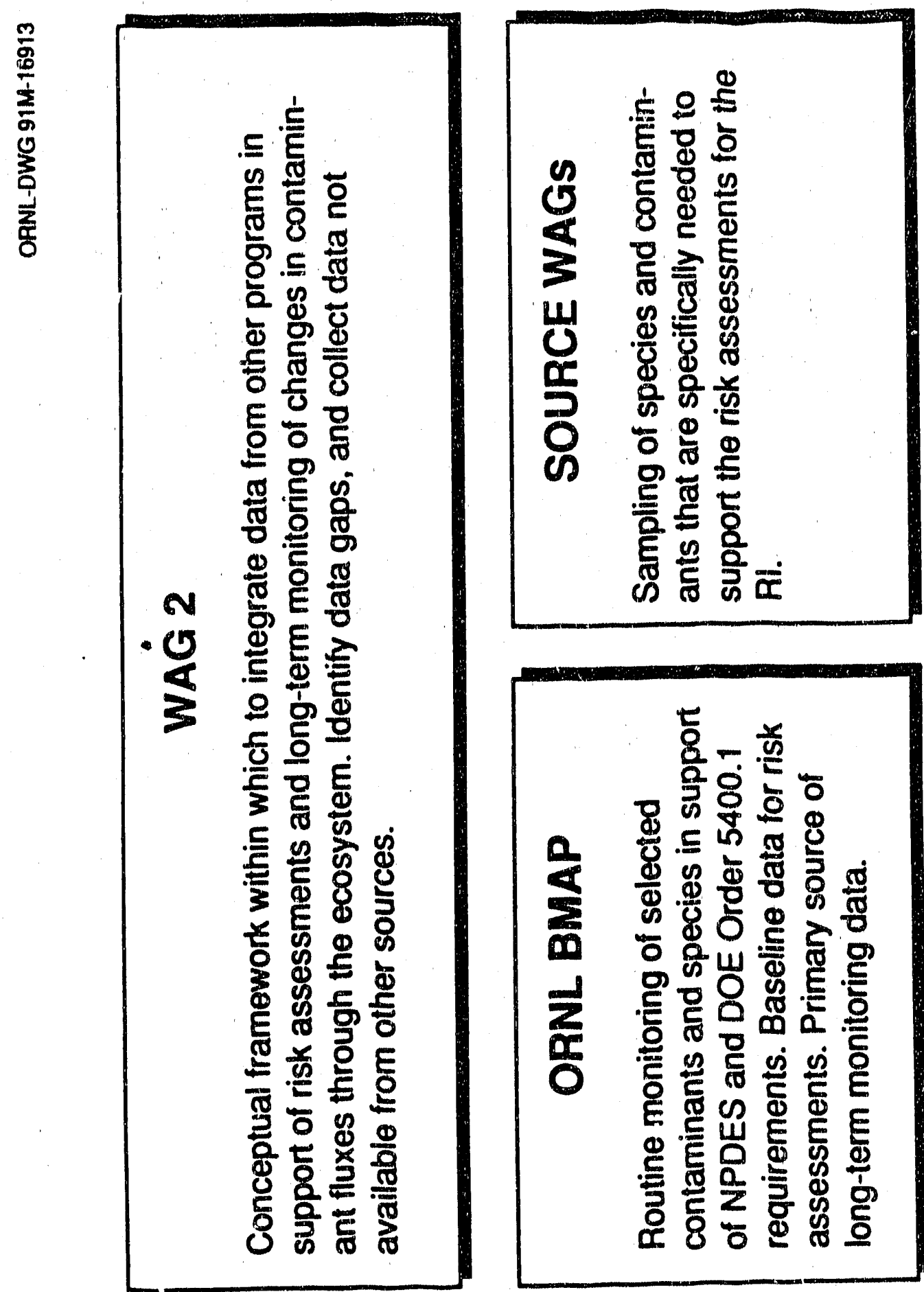

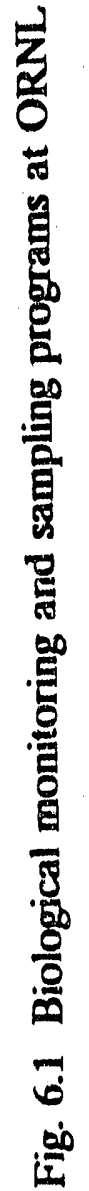


hazards at the site, and logically related to the remedial investigation (Suter 1989). Measurement endpoints must correspond to or predict an assessment endpoint and must obviously be readily measurable. They should also be appropriate to the scale of the site and the exposure pathway (Suter 1989).

Assessment endpoints typically relate to populations or higher levels of biological organization. The exception to this occurs with rare or endangered species, where each individual is assumed to be important to the survival of the species. An assessment endpoint may also be related to human health effects.

The RI plan for WAG 2 (ORNL 1990) identifies five assessment endpoints: (1) $\geq 10 \%$ reduction ${ }^{1}$ in the abundance or production of the local populations of any fish species, (2) $\geq 10 \%$ reduction $^{1}$ in the abundance or production of the local populations of any bird species, (3) $\geq 10 \%$ reduction $^{1}$ in the abundance or production of the local populations of any wild mammal species other than small rodents, $(4) \geq 10 \%$ reduction $^{1}$ in the production of any local plant population, and (5) any toxic effect on individuals ${ }^{2}$ of an endangered species sufficient to impair survival or reproduction. An additional endpoint for this assessment is any biological pathway resulting human consumption of contamination that presents a human health risk $>10^{-4}$.

\subsection{REVIEW OF EXISTING DATA}

The remedial investigation plan for WAG 2 (ORNL 1990) contains an excellent review of the existing literature on contaminants within the biota. A summary of this information as it applies to ecological risk assessment is also included in the screening-level risk assessment for WAG 2 (Blaylock et al. 1991b). Only the conclusions of these studies are presented in this section.

The screening-level ecological risk assessment for WAG 2 (Blaylock et al. 1991b) identifies PCBs and mercury as the principal contaminants of concern. Piscivorous predators are the major biological group at risk. However, there are a number of gaps in the data on

\subsubsection{Conceptual Model}

Water is the primary vector of contamination in the WOC watershed. Thus, aquatic biota and those organisms that feed on aquatic organisms are the principal receptors. It is

${ }^{1}$ The $10 \%$ level of population effects was chosen as approximately the detection limit of field measurement techniques and is probably below the detection limits of the public (i.e., fishermen would probably not notice a $10 \%$ reduction in the abundance of tish).

2 The endpoint is defined at the individual level for endangered species because of the greater legal and societal concern for these species. nonradiological contaminants that could alter these results. Ecological risks from radioactive contaminants have not been addressed in the screening-level ecological risk assessment for two reasons: (1) available data from other studies suggest that ecological risks from radiological contaminants are unlikely to be significant unless levels are such as to pose a significant risk to humans and (2) radioactive contaminants have been extensively addressed in the screening level human health ris $k$ assessment. 
also possible for organisms burrowing and feeding in contaminated soils to accumulate contaminants and for these contaminants to be concentrated through the food chain. The potential for uptake and accumulation varies considerably among contaminants.

Macintosh et al. (1991) have developed a model of PCBs and mercury movement through the biological system to two key predators (mink and great blue herons). This model incorporates current ecological information on transfer of these two contaminants through a complex food web that is typical of WAG 2 (Fig. 6.2). Both terrestrial and aquatic pathways are inrluded in the model.

Such a model, when expanded to include other contaminants and target species, provides the framework for evaluating data needs in support of risk assessments. The model can also be used to focus characterization efforts on those few areas where data are lacking. The model can be cxpanded to evaluate the potential risk to a wider array of organisms, leading to identification of those organisms most useful for long-term biological monitoring.

\section{Radiological Contaminants}

Ec Jlogical risks from radioactive contaminants have not been addressed in the screening-level ecological risk assessment for two reasons: (1) available data from other studies suggest that ecological risks from radiological contaminants are unlikely to be significant unless levels are such that they pose a significant risk to humans and (2) radioactive contaminants have been extensively addressed in the screening-level human health risk assessment. Nevertheless, radioactive contaminants in biota are an important component of the ecological assessment berause they represent a potential pathway for human health risks.

The WAG 2 RI Plan (ORNL 1990) contains a review of existing information on radiological contamination within the WOC watershed. Three radionuclides $\left({ }^{60} \mathrm{Co}\right.$, ${ }^{90} \mathrm{Sr}$, and ${ }^{137} \mathrm{Cs}$ ) have been measured at above background levels in aquatic biota (ORNL 1990, Sect. 6.6). Plutonium-239 has been measured burrowing crayfish in the area (Delaney et al. 1979).

Terrestrial vegetation contains ${ }^{3} \mathrm{H},{ }^{90} \mathrm{Sr}$, and ${ }^{99} \mathrm{Tc}$ at levels above background. Cobalt-60 and ${ }^{137} \mathrm{Cs}$ are also taken up by terrestrial vegetation, but not at levels greater than soil concentrations. A few deer taken in managed hunts on the Oak Ridge Reservation have contained measurable concentrations of ${ }^{90} \mathrm{Sr}$, and ${ }^{90} \mathrm{Sr}$ has been measured in small rodents in WAG 4 adjacent to WAG 2. Small rodents and larger animals in the floodplain also contained elevated concentrations of ${ }^{137} \mathrm{Cs}$. Unpublished data suggest that ${ }^{129} \mathrm{I}$ may be present in thyroid glands of deer on the Oak Ridge Reservation, but it is unclear whether these one-time measurements reflected a single-release episode or a continuing source (J. W. Evans, Tennessee Wildlife Resources Agency, personal communication, to T. L. Ashwood, ORNL 1991; C. T. Garten, ORNL, personal communication, to T. L. Ashwood, ORNL. 1991). Analytical difficulties have precluded extensive measurement of ${ }^{99} \mathrm{Tc}$ in biota, and other radionuclides may be present at trace levels. 
$\frac{2}{2}$
$\frac{8}{5}$
8
8
8

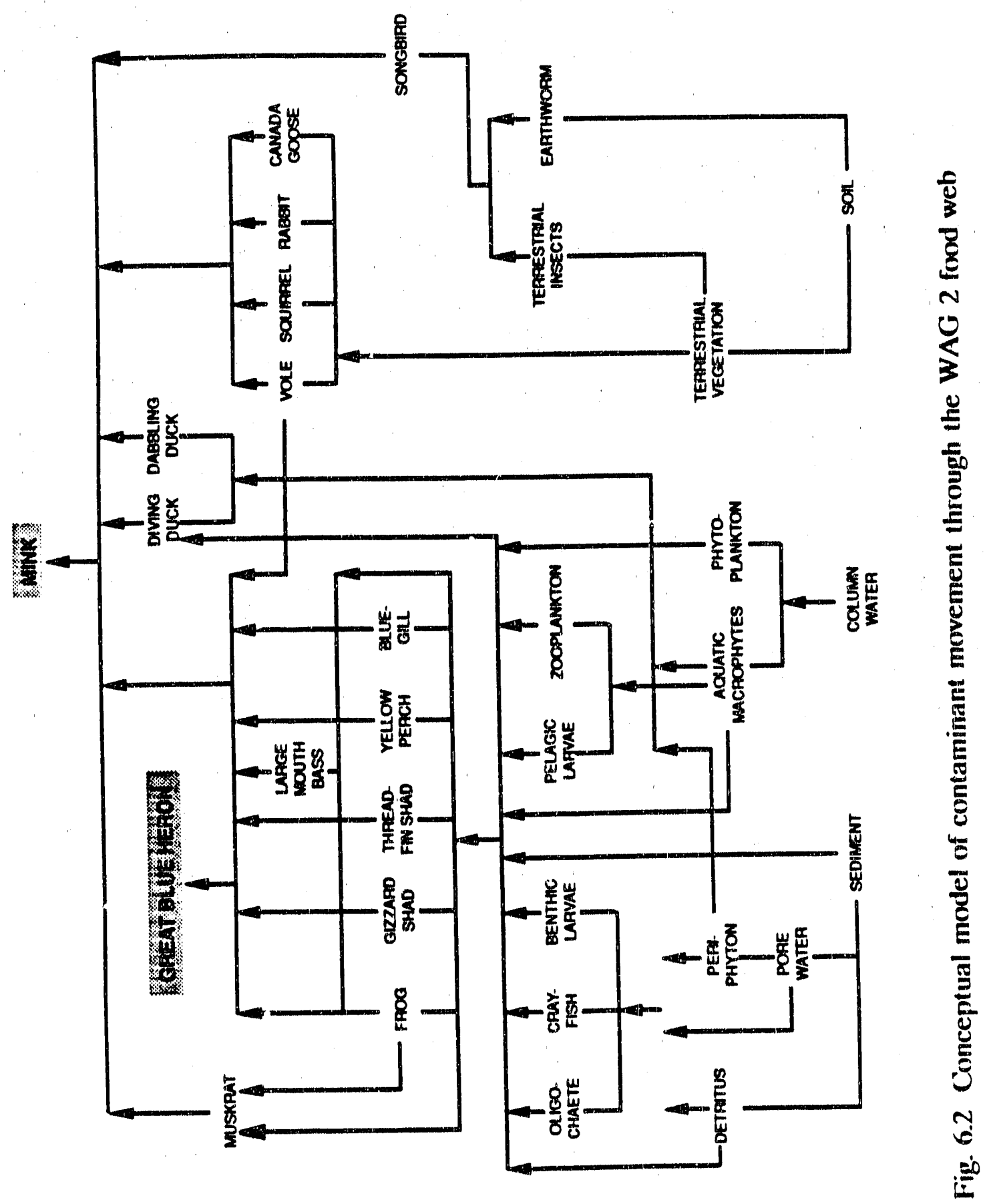




\subsection{Nonradiologicul Contaminants}

BMAP data suggest that mercury, PCBs, and chlordane are the primary contaminants accumulating to above background levels in aquatic biota (ORNL 1990, Sect. 6.6). Recent studies of mallard ducks have shown that these migratory waterfowl accumulate mercury and other trace metals during their brief stopovers on WOL (Loar 1991).

Little information is available on nonradioactive contaminants in the terrestrial biota of WAG 2. Meyers-Schone (in Loar 1991) found concentrations of PCBs in two species of turtles in WOL. Indications of PCBs and mercury have been found in eggs of a great blue heron rookery near the K-25 site ( $R$. S. Halbrook, ORNL, personal communication to T. L. Ashwood, ORNL, 1991). Herons from that rookery feed in WAG 2, but there are also other possible sources of the PCBs and mercury.

\subsubsection{Data Needs}

For the model developed by MacIntosh et al. (1991) to serve as a conceptual framework for the ecosystem in WAG 2 , it must be expanded to include all contaminants of concern and all species potentially at risk. The only nonradiological contaminant of concern not already included is chlordane. Our current best knowledge suggests that radiological contaminants are not of concern from an ecological risk perspective; however, our model must provide insight into the biological transfer of radioisotopes that may be of concern from a human health perspective. Thus, the model must be expanded to include chlordane, ${ }^{90} \mathrm{Sr}$, and ${ }^{137} \mathrm{Cs}$ and perhaps additional food-web pathways.

The BMAP is evaluating raccoons and screech owls as possible biological monitoring species. If these two species show elevated levels of the contamiuants of concern, they will be added to the model. Similarly, the WAG 5 biota sampling plan includes evaluation of contaminant levels in wild turkeys. If turkeys from WAG 5 contain elevated levels of the contaminants of concern, it is likely that turkeys in WAG 2 would also contain contamination above background levels and the model would be expanded to include turkeys. Although these two studies are aimed at specific animals and have differing goals, a more systematic method of identifying potentially contaminated wildlife is needed to ensure that at risk organisms and potential pathways to humans are not overlooked.

The screening level ecological risk assessment suggests that mink and kingfishers could be at risk from contaminant levels known to be present in aquatic biota in WAG 2. (Blaylock et al. 1991b). Data are needed, therefore, to determine whether these animais are in fact iffecteci'.

Although some sediment contamination data exist and more are being collected, the ecological risk assessment identifies a need for measuring contaminant levels in the interstitial waters of fine sediments within WOC and WOL. Such levels are more directly related to biological exposure than are contaminant levels adsorbed to sediment particles (Blaylock et al. 1991b). Until more data are available on sediment contamination (see Sect. 3 of this report), we believe that fish and clam bioaccumulation data being collected by BMAP adequately address the question of which contaminants are available for uptake by biota and the degree to which these contaminants are being incorporated into the food-web. 
The screening level risk assessment also identifies the need for surveys of WAG 2 to identify threatened or endangered species (Blaylock et al. 1991b).

As data from soil/sediment, groundwater, and surface water sampling are obtained, it may become necessary to add to the list of contaminants of concern. To determine the need for additional biological sampling, two screening steps will be taken. First, we will conduct toxicity tests to determine whether soil or water samples from contaminated areas are toxic to standard test organisms. Second, and based in part on the toxicity tests, we will update the screening level risk assessment to determine whether the identified contaminants pose a threat to biota or human health.

\subsection{OBJECTIVES}

Based on the data needs identilied in Sect. 6.2.4 and the overall aims identified in Sect. 6.1, the following specific objectives have been identified.

1. Develop and maintain a computerized model of contaminant flow through the WAG 2 ecosystem that identifies organisms at risk and important pathways to humans and which can be used to predict effects of changes in contaminant inputs to the system.

2. Coordinate efforts of the ORNL BMAP and biological sampling programs in source WAGs to provide an integrated long-term monitoring program to measure the impacts of changes in contaminant inputs to the system.

3. Obtain data on contaminant levels in organisms that are identified as being at risk and that are not already sampled in other programs.

4. As information is obtained from other sampling tasks within the WAG 2 RI and within the BMAP, determine the impact on biota and the need for additional sampling.

\subsection{BIOLOGICAL SAMPLING}

Most of the biological sampling for ORNL is expected to occur through the BMAP and RIs/RFIs for the source WAGs. BMAP bioaccumulation studies include sampling of clams and fish in all reaches of WOC and Melton Branch. These samples are analyzed for radionuclides, metals, and important organic compounds. In addition, ambient toxicity testing is conducted approximately bimonthly at locations throughout the WOC/Melton Branch system. Source WAGs will include sampling of biota that are relevant measurement endpoint for the WAG [e.g., wild turkeys in WAG 5 (Ashwood 1991)], and the ecological assessments for these source WAGs will include ambient toxicity testing of highly contaminated seeps, stream reaches, and soils.

The primary role of the ecological assessment of WAG 2 is to provide a framework within which to integrate these data. However, some data that are needed will not fall within the purview of other programs; therefore, some biological sampling within the WAG 2 RI needed.

In the short-term, data are needed to determine whether the conceptual model should be expanded to include additional pathways. Also, short-term data are needed to determine 
the status of species already identified as potentially at risk and to determine whether there are state-listed threatened or endangered species in WAG 2 (ORNL 1990, Sect. 8.1.3.6).

Over the longer term, it will be necessary to integrate data from the BMAP and to provide guidance to the BMAP and source WAG RIs on additional data needs. As data are obtained that suggest additional contaminants of concern, toxicity tests and risk evaluations will be required to determine what changes, if any, are required in the model. Finally, the model will be used to predict the ecological impacts associated with remedial actions both within WAG 2 and within source WAGs, where those remedial actions are expected to change contaminant inputs to WAG 2.

These short- and long-term activities are described in the following tasks.

\subsubsection{Task 1: Scoping Survey of Contaminant Levek in Selected Wildlife}

This task involves collecting fecal material (scats) from various wildlife species within WAG 2 and analyzing the material for contaminants of concern. Some fraction of the contaminants that pass through the gut of an animal is absorbed by the animal, and the remainder is excreted. The fraction absorbed varies with the type of animal and the chemical form of the contaminant. Nevertheless, some fraction of the contaminant will be excreted. Comparison of contaminant levels between scats in WAG 2 and those from pristine areas can be used to evaluate whether a specific contaminant is present at elevated levels in animals in WAG 2.

This is a task that will begin in FY 1992 and will continue for 2 years. It is anticipated that this period of time will be required to collect sufficient samples from WAG 2 and pristine areas to provide statistically significant results. At this time it is not possible to determine the exact number of scats that may be available, but we anticipate that at least 25 samples of each species from WAG 2 and 25 samples of each species from a pristine area will be required to provide a low enough standard error to evaluate differences in contaminant levels. If variability is higher than anticipated, more samples may be required. Scats will be gamma counted for ${ }^{137} \mathrm{Cs}$ and analyzed for PCBs and metals (including mercury).

At this time, only three species are being included in this study: striped skunks and opossums feed on terrestrial invertebrates that may be contaminated through ingestion of contaminated soil, and muskrats consume plant and detrital material from the aquatic system. As noted earlier, raccoons, turkeys, and screech owls are already being evaluated. Mink, great blue herons, and kingfishers are already incorporated in the model. Other predators in the area (coyotes, grey and red foxes, and bobcats) feed primarily on small herbivorous mammals and are unlikely to accumulate significant levels of the contaminants of concern.

This task supports Objectives 1 and 3 in Sect. 6.3. 


\subsubsection{Task 2: Threatened and Endangered Species Surveys}

Although several federally listed threatened or endangered species occur in the region (Krondsma 1987), none are known to occur in WAG 2. Several state-listed species occur on the ORR and may occur in WAG 2. WAG 2 will be surveyed to determine whether state-listed species are present or are likely to be present. The surveys will be conducted by biologists familiar with the species that may potentially be expected to live in habitats similar to WAG 2, and they vill be conducted during times of the year when the species are most likely to be identifiable. The surveys will only provide evidence of presence or absence, no quantitative information on population status will be obtained. This survey will be completed in FY 1992, and any species identified will be evaluated for inclusion in the model.

This task supporis Objective 1 in Sect. 6.3.

\subsubsection{Task 3: Mink and Kingfisher Sampling}

WAG 2 contains habitat suitable for mink and kingfishers. The screening-level risk assessment (Blaylock et al. 1991b) identifies these piscivorous predators as being at risk from PCB and mercury levels in their prey. There is even evidence (Macintosh et al. 1991) that mink may be consuming prey with PCB and mercury levels sufficient to impair reproduction.

The objective of this task is to collect data on PCB and mercury levels in mink and kingfishers in WAG 2. However, it is possible that no viable populations of these animals exist in WAG 2.

In conjunction with the avian portion of the threatened and endangered species survey, a survey will be made to determine whether kingfishers are present in the WAG. Similarly, the raccoon study being conducted as part of the BMAP involves live trapping of raccoons and scent station surveys to determine the relative abundance of raccoons. Both the live trapping and scent station surveys will also provide evidence of the existence of mink in WAG 2.

If mink and kingfishers are found in WAG 2 in sufficient quantities to provide statistically meaningful results, samples will be collected of mink fur and kingfisher feathers for mercury and trace metal analysis. Mink scat and kingfisher droppings will be analyzed for PCBs, and each trapped mink will be biopsied to provide sufficient tissue for PCB analysis. Captured minks will be ear-tagged so that subsequent trapping can be used to determine whether mink in the area are residents or merely transients. We anticipate collecting a minimum of 3 and a maximum of 10 samples for each species and contaminant over a period of 2 to 3 years.

Once scent station transects have established in the WAG 2 area, they will be run at least twice a year for the duration of live WAG $2 \mathrm{RI}$ to determine changes in relative abundance of wildlife in the area. It is anticipated that effective remedial action in source WAGs and in WAG 2 should result in an increase in the abundance of sensitive species such as the mink. 
This task addresses Objective 3 in Sect. 6.3.

\subsubsection{Task 4: Contaminant Fate Model}

The model developed by MacIntosh et al. (1991) uses the concentrations of specific contaminants in soil, water, and sediment as input parameters. Thus, the biological effects of contaminant levels in specific WAGs or the impact of changes in contaminant levels due to remedial actions or storms can be evaluated.

The model will be expanded as already discussed based on input from Tasks 1 through 3 and from the BMAP and source WAG investigations. In addition, sensitivity studies on key model parameters may indicate the need for additional information on the transfer of contaminants at selected points within the modeled food web. Most of the data required to expand and improve the model is expected to be available through the literature. However, it may be necessary to conduct specific small-scale investigations to augment the literature. As these needs are identified, they will be submitted for approval as extensions of this plan.

The primary objective of this task is to expand the model as needed and to use the model to suggest changes in the BMAP, determine the need for specific investigations in source WAGs, support ecological risk assessments, and evaluate proposed remedial actions.

This task supports Objectives 1, 2, and 4 in Sect. 6.3.

\subsubsection{Task 5: Toxicity Testing and Risk Review}

The purpose of this task is to evaluate the biological impact of new data generated from soil, sediment, and water sampling in the RIs for source WAGs and from other portions of the WAG 2 RI. As these new data suggest that additional contaminants should be addressed or that levels of contaminants are much different than previously measured, we will perform toxicity tests and risk assessments to determine whether the contaminants pose a risk to biota or human health.

This task addresses Objective 4 in Sect. 6.3.

\subsection{QUALITY ASSURANCE}

Table 6.1 presents by task the samples that will be taken and the analyses to be performed. Sampling procedures will be based on proven wildlife sampling methods and will be documented separately. No EPA-approved methods exist for the the analyses required. However, we will use existing procedures developed in support of the BMAP and the Clinch River RI, and we will document these procedures separately. Procedures for sample collection are presented in Appendix C. All procedures for sample collection and analysis will be reviewed by the ERD-APO. 
Table 61. Samples and analyses to support biological sampling in WAG 2

\begin{tabular}{|c|c|c|c|c|}
\hline & Task & Sample matrix & No. of samples & Analyte(s) \\
\hline 1. & Scoping survey & Fecal material & $\geq 150$ & $\begin{array}{l}{ }^{137} \mathrm{Cs}, \mathrm{PCBs}, \\
\text { Metals, } \mathrm{Hg}\end{array}$ \\
\hline 2. & $\begin{array}{l}\text { Threatened \& enda } \\
\text { species survey }\end{array}$ & gered & None & \\
\hline 3. & $\begin{array}{l}\text { Mink \& kingfisher } \\
\text { survey }\end{array}$ & $\begin{array}{l}\text { Mink hair } \\
\text { Mink tissue } \\
\text { Mink scat } \\
\text { Kingfisher feathers } \\
\text { Kingfisher droppings }\end{array}$ & $\begin{array}{l}\leq 10 \\
\leq 10 \\
\leq 10 \\
\leq 10 \\
\leq 10\end{array}$ & $\begin{array}{l}\text { metals, } \mathrm{Hg} \\
\text { PCBs } \\
\text { PCBs } \\
\text { metals, } \mathrm{Hg} \\
\text { PCBs }\end{array}$ \\
\hline 4. & Model & & None & \\
\hline 5. & Toxicity tests & Water, soil, sediment & unknown & toxicity \\
\hline
\end{tabular}

- Toxicity tests will be conducted using EPA-approved methods for water (Ceriodaphnia dubia) and soil (seedlings and earthworms). 


\title{
7. SAMPLE TRACKING AND RECORDS MANAGEMENT
}

\author{
J. A. Watts
}

\subsection{INTRODUCTION}

The WAG 2 RI will generate data from many sources, including field measurements, laboratory analyses of environmental samples, and output from simulation models. The field and simulation modeling studies proposed in this S\&A plan have been designed to fill data gaps, quantify the complex interactions between contaminants and the environs in and surrounding WAG 2, and provide information for risk assessment activities. Specific data needs are discussed in Sects. 2 through 6 of this report. Important records regarding the collection, analysis, and disposition of the samples and data will also be generated. The sample tracking and records management procedures for WAG 2 data collection are described in this section.

\subsection{OBIECTIVES}

The objectives of the sample traceability and records management plan are to provide organization, protection, retrievability, and accountability of the data and documents generated during the S\&A phases of the WAG 2 RI. All documents required to provide a complete and accurate history of data collection and analyses will be included in the records management system (EPA 1988a). The procedures in this plan are necessary to ensure that such data and records are documented to the extent necessary to support any future legal or administrative actions. The data management procedures will ensure that data are made available for uses such as statistical analysis and modeling without the introduction of additional uncertainty fres data entry and management activities.

This plan identifies the documents, records, and data to be retained and describes the management structure and organization necessary to ensure their integrity and retrievability. Three general types of information and documents will constitute the necessary WAG 2 RI record.

1. Records-any documentation (printed material) that must be maintained permanently unless directed otherwise by project management.

2. Nonrecord material-documentary materials that have little or no value from an overall operational standpoint and are used for short-term reference purposes.

3. Data-primarily numerical results obtained from environmental monitoring and sampling activities.

Record materials will be maintained in the ERD/DMC, as described in the WAG 2 RI plan (ORNL 1990). Numeric data are maintained in the numeric data base (NDB) (Sect. 7.6). 


\subsection{FIELD DATA MEASUREMENTS AND OBSERVATIONS}

Field notes are the primary record that will provide sufficient data and observations to enable reconstruction of events that occurred. Field notebook entries should be factual, detailed, and objective. All field measurements, such as $\mathrm{pH}$, temperature, conductivity, water flow, sediment characteristics, and instrument calibration data, will be recorded in the field notebocks or on specially designed data forms. All data will be directly entered in the field, signed, and dated. Changes made to original notes should not obliterate the original information. All field data records will be organized into standard format when possible.

\subsubsection{Logbooks}

Bound, registered logbooks with numbered pages will be used to record all information related to the collection, handling, and sequentially numbered current location of samples. Data will be recorded directly in an official logbook, onto a sequentially numbered data form, or electronically using data logger equipment. Charts and other printed material that will fit into the logbook will be glued or otherwise permanently fastened in the logbook. Oversize pages will be kept in a designated place, which will be referenced in the logbook. All logbook entries will be made in indelible ink; a single line will be drawn through errors, and the date and initials of the individual making the correction will appear alongside the drawn line (ESP-500, Rev. 1, Kimbrough et al. 1990). The field sampling coordinator will be the custodian of the logbook and will review entries daily. Each page will be signed by the recorder on completion of that page of entries.

\subsubsection{Sample Custody}

Sample custody will be documented from the time the sample is collected in the field until final disposition. Documentation will trace sample possession through all transfers of custody. A sample will be considered to be under a person's custody if

1. the sample is in the person's physical possession,

2. the sample is in the line of sight of the person after taking possession,

3. the sample is secured by that person so that any tampering can be detected, or

4. a sample is secured by the person in possession in an area which only authorized personnel can enter.

All sample custody procedures will be in accordance with ESP-500 (Rev. 1), Manual Chain of Custody Procedures (Kimbrough et al. 1990). A chain-of-custody form will accompany all samples as transfers from one custodian to another occurs. The chain-ofcustody form will be signed and dated by both the individual transferring custody and the individual accepting custody. It is the responsibility of the individual investigators and the WAG 2 RI project manager to safeguard these records. The WAG 2 RI project manager will forward field notebooks to the DMC at the conclusion of field activities. 


\subsection{ANALYTICAL LABORATORY DATA}

The Data Management Team Leader will establish and maintain a forms control system for chain of custody and requests for analytical services. These forms will be completed by the sample custodian and transferred to the ASC along with the samples for delivery to the appropriate analytical laboratory. Custody will be transferred to the analytical laboratory supervisor or his/her appointed designee. Upon final analyses of the samples, a copy of the completed chain-of-custody form will be returned to the WAG 2 RI project manager for submittal to the DMC.

The contract analytical laboratories will be requested to use a computerized laboratory management system to track samples and report analytical results. Completed and approved data files will be electronically copied to ORNL computers. Transfers will be accomplished with software that incorporates accepted error-checking protocols as described in Sect. 2.5.14.3. Data will be included in the ORNL ER NDB only after the hard copy of such data has been submitted by the WAG 2 RI project manager to the DMC.

Results of any water quality analyses performed at the Aquatic Ecology and Radiological Laboratories of ESD will be recorded directly on sequentially numbered data sheets, reviewed by the analysts and signed, and copies will be sent to data entry, where data will be double entered and verificd to ensure accurate entry.

\subsection{SIMUI ATION AND RISK ASSESSMENT MODEIS AND DATA}

Documentation of contaminant fate and transport models will be retained. Documentation includes descriptions of the model equations, assumptions, solution methods, and initial and boundary conditions used. The input data, output, and program files for calibration and for analyses used as bases for important programmatic decisions will be available to outside reviewers. All data input, output, program files, equations, factors, and assumptions employed in the screening-level risk analyses and in subsequent risk analyses conducted for the WAG 2 RI project will also be retained and available to outside reviewers. Custody of all data, computer programs, and results from simulation models will be maintained by the task leader or appointed designee. This information will be transforred to the WAG 2 RI pruject manager for transmittal to the DMC at the completion of the task.

\subsection{DATA MANAGEMENT}

Data management for this project will utilize the NDB of the ORNL ER Program (Voorhees et al. 1988, 1989; Hook ef al. 1990) for management and storage of field, analytical, and simulation model data. The principal steps for processing data to be included in the NDB are identified in Fig. 7.1. These procedures will be followed for entry and verification of all numeric data generated during the S\&A phases of the WAG 2 RI. Data management activities, reference to programs used to manipulate the data, and documentation to the ciata base are recorded in technical notebooks (QA-ES-6-100, Roberson and Logdson 1989). Changes to the data base, initiated by the analytical laboratory, will be provided in writing. Correction to the data base initiated by the WAG 2 RI staff will be submitted in writing to the appropriate analytical laboratory. They shall be responsible for subsequent 


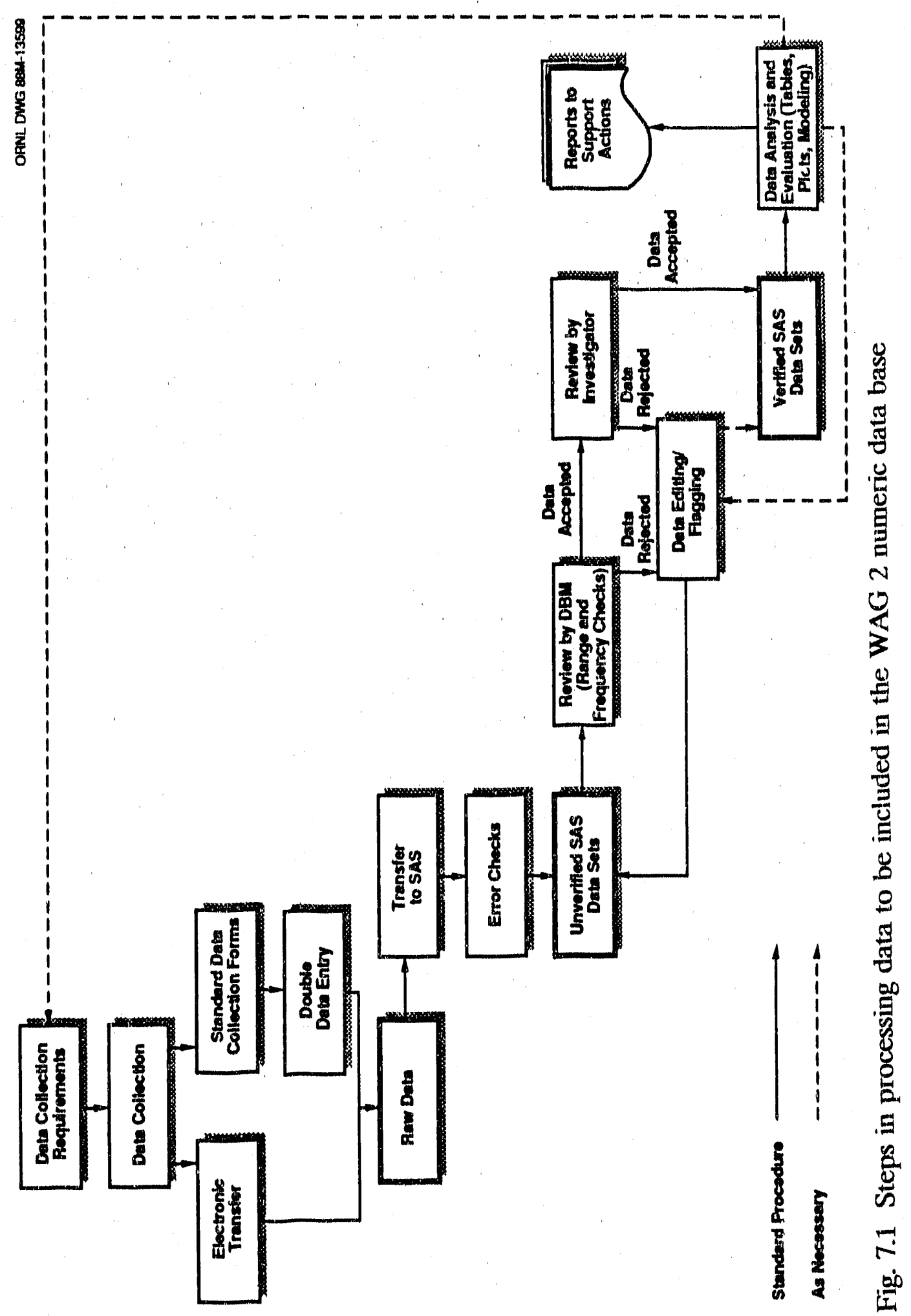


verification, correction, and reissuance of the data in question. Requests for direct "read only" access to WAG 2 RI data or hard copy and graphical output will require the approval of the WAG 2 RI project manager.

Table 7.1 summarizes the WAG 2 S\&A data to be included in the NDB. Numeric data are manipulated primarily with SAS software and stored as SAS data sets. SAS and other compatible products (e.g., EXCEL, Lotus 1-2-3, and dBase IV) are utilized with individual personal computers. Data collected in this project will be submitted to the Oak Ridge Environmental Information System (OREIS) when validation procedures are completed.

The ERD DMC will be used for management of record material. The DMC has a computerized index for retrieval of information generated by the program (e.g., correspondence, project plans, field notebooks, chain-of-custody records, and hard-copy data reports) from serially numbered archived files. All records will be permanently retained. Data management activities will support the preparation of all documents required for the administrative record.

Field data will be reviewed by the field sampling coordinator and the QAVQC coordinator, who will be responsible for resolving any questions regarding the data.

Laboratory data will be checked for both internal consistency and transmittal errors by the laboratory supervisor. Errors will be corrected and documented. The analytical data reports will be reviewed by using the quality assurance/quality control procedures outlined Sect. 2.5. 
Table 7.1. Data to be included in the WAG 2 sampling and analysis aumeric data base and the originating office"

(All data are to be transmitted to the WAG 2 RI project manager for inclusion in the Numeric Data Base.)

\begin{tabular}{|c|c|c|}
\hline Record origination & Category of data record & Comment \\
\hline Biota Sampling Team & $\begin{array}{l}\text { Organism vital statistics } \\
\text { ESD laboratory data }\end{array}$ & $\begin{array}{l}\text { Recorded in field } \\
\text { notebooks, duplicates } \\
\text { transferred to Project } \\
\text { Manager }\end{array}$ \\
\hline Sediment Sampling Team & $\begin{array}{l}\text { Sediment characteristics } \\
\text { Water quality data } \\
\text { Water flow rate data } \\
\text { Sediment core length } \\
\text { ESD laboratory data }\end{array}$ & $\begin{array}{l}\text { Recorded in field } \\
\text { notebooks, data forms } \\
\text { duplicates to Projeci } \\
\text { Manager }\end{array}$ \\
\hline $\begin{array}{l}\text { Surface Water Sampling } \\
\text { Team }\end{array}$ & $\begin{array}{l}\text { Water quality data } \\
\text { Field turbidity data } \\
\text { Water flow rate data } \\
\text { ESD laboratory data }\end{array}$ & $\begin{array}{l}\text { Recorded on standard } \\
\text { field data sheets, } \\
\text { duplicates to Project } \\
\text { Manager }\end{array}$ \\
\hline $\begin{array}{l}\text { Groundwater Sampling } \\
\text { Team }\end{array}$ & $\begin{array}{l}\text { Water level data } \\
\text { Water quality data } \\
\text { Hydraulic conductivity data } \\
\text { Groundwater geochemistry data } \\
\text { Physicochemical data } \\
\text { Contaminant diffusion data }\end{array}$ & $\begin{array}{l}\text { Recorded on standard } \\
\text { field data sheets, } \\
\text { duplicates to Project } \\
\text { Manager }\end{array}$ \\
\hline $\begin{array}{l}\text { Data Management, } \\
\text { Integration, and Remedial } \\
\text { Action Evaluation Task }\end{array}$ & $\begin{array}{l}\text { Contaminant fate and transport } \\
\text { model data }\end{array}$ & $\begin{array}{l}\text { Retention of model } \\
\text { input, output, and } \\
\text { calibration data }\end{array}$ \\
\hline $\begin{array}{l}\text { Contract Analytical } \\
\text { Laboratory }\end{array}$ & Analytical and QA data & $\begin{array}{l}\text { Electronic transfer to } \\
\text { NDB, originals to } \\
\text { Project Manager }\end{array}$ \\
\hline
\end{tabular}

"Subject to final approval. 


\section{REFERENCES}

Amano, H., C. T. Garten, Jr., and R. D. Lomax. 1987. A field survey of environmental tritium in areas adjacent to ORNL solid-waste storage areas. ORNL/TM-10438. Oak Ridge National Laboratory, Oak Ridge, Tennessee.

ANSI/ASME (American National Standards Institute/American Society of Mechanical Engineers). 1986. Quality assurance program requirements for nuclear facilities. ANSI/ASME NQA-1-1986 (and current amendments). New York.

American Society of Civil Engineers, 1975. Sedimentation Engineering. (Ed. by V.A. Vanoni), ASCE, New York. 745 pp.

Ashwood, T. L., D. S. Wickliff, and C. M. Morrissey. 1991. Active sites environmental monitoring program: Mid-FY 1991 reprort. ORNL/M-1442. Oak Ridge National Laboratory, Oak Ridge, Tennessee.

ASTM (American Society for Testing and Materials). 1990. Annual Book of ASTM Standards: Section 11, Water and Environmental Technology, Vol. 11.02, D4411-84, Sampling Fluvial Sediment in Motion. New York.

BNI, Inc. 1989. Sample information management system (SIMS). Document No. 1603. Bechtel National, Inc., Oak Ridge, Tennessee.

Blaylock, B. G., M. L. Frank, L. A. Hook, and F. O. Hoffman. 1991a. Interim Site Characterization and Contaminant Screening Report on the Whiteoak Creek Embayment. Draft Report. Oak Ridge National Laboratory, Oak Ridge, Tennessee.

Blaylock, B. G., M. L. Frank, G. W. Suter, L. A. Hook, and J. A. Watts. 1991b. Screening of Contaminants in Waste Area Group 2 at Oak Ridge National Laboratory (White Oak Lake, White Oak Creek, and Melton Branch). ORNL/ER-62. Oak Ridge National Laboratory, Oak Ridge, Tennessee.

Borders, D. M., S. M. Gregory, R. B. Clapp, B. J. Frederick, G. K. Moore, J. A. Watts, C. C. Broders, and A. T. Bednarek. 1991. Annual Hydrologic Data Summary for the Whiteoak Creek Watershed: Water Year 1990 (October 1989-September 1990). ORNL/ER-55. Oak Ridge National Laboratory, Oak Ridge, Tennessee.

Cerling, T. E. 1986. Investigation of bedload transport of contaminated gravel in the White Oak Creek drainage. Subcontract No. 19X-27463C. Department of Geology and Geophysics, University of Utah, Salt Lake City.

Cerling, T. E., and B. P. Spalding. 1981. Areal distribution of ${ }^{60} \mathrm{C},{ }^{137} \mathrm{Cs}$, and ${ }^{90} \mathrm{Sr}$ in streambed gravels of White Oak Creek watershed, Oak Ridge, Tenn. ORNL/TM-7318, Oak Ridge National Laboratory, Oak Ridge, Tennessee. 
Cerling, T. E. and B. P. Spalding. 1982. Distribution and relationship of radionuclides to streambed gravels in a small watershed, Environ. Geol. 4: 99-116.

Cerling, T. E. and R. R. Turner. 1982. Formation of Freshwater Fe-Mn coatings on gravel and the behavior of ${ }^{60} \mathrm{Co},{ }^{90} \mathrm{Sr}$, and ${ }^{137} \mathrm{Cs}$ in a small watershed," Geochim. et Cosmochim. Acta 46: 1333-43.

Clapp, R. B., D. M. Borders, R. Drier, T. A. Fontaine, G. K. Moore, D. K. Solomon, J. A. Watts, and D. S. Wickliff. 1991. ORNL Environmental Restoration Monitoring and Assessment Program-Annual Report, FY 1991: Monitoring Plan. ORNL/ER-54. Oak Ridge National Laboratory, Oak Ridge, Tennessee. (in press)

Delaney, M. S., R. C. Dahlman, and R. B. Craig. 1979. An investsigation of plutonium concentration and distribution in burrowing crayfish from White Oak Creek floodplain. ORNL/TM-6702. Oak Ridge National Laboratory, Oak Ridge, Tennessee.

DOE (Department of Energy). 1988. Historical radionuclides releases from current DOE Oak Ridge Opearations Office facilities. U.S. Department of Energy, Oak Ridge Operations Office, Oak Ridge, Tennessee, pp. 36.

Duguid, J. O. 1975. Status report on radioactivity movement from burial grounds in Melton and Bethel valleys. ORNL/TM-5017. Oak Ridge National Laboratory, Oak Ridge, Tennessee.

Emmett, W. M. 1981. Measurement of Bedload in Rivers. IN: Erosion and Sediment Transport Measurement, Proceedings of Symposium, Florence, Italy. IAHS Publication No. 133.

Energy Systems. 1992. 1990 Clinch River RCRA facility investigation (CRRFI) plan. ES/ER-1/D1, Martin Marietta Energy Systems, Inc., Oak Ridge National Laboratory, Oak Ridge, Tennessee.

EPA (U.S. Environmental Protection Agency). 1979a. Handbook for Analytical Quality Control in Water and Wastewater Laboratories. Office of Research and Development, Cincinnati, Ohio.

EPA (U.S. Environmental Protection Agency). 1979b. Radiochemical analytical procedures for analysis of environmental samples. EMSL-LV-0539-17. Environmental Monitoring and Support Laboratory, Las Vegas, Nevada.

EPA (U.S. Environmental Protection Agency). 1980a. Interim guidelines and specification for preparing quality assurance project plans. EPA QAMS-005/80. Office of Monitoring Systems and Quality Assurance, Washington, D.C.

EPA (U.S. Environmental Protection Agency). 1980b. Interim methods for the sampling and analysis of priority pollutants in sediments and fish tissues. EPA 600,4-81-055. Environmental Monitoring and Support Laboratory, Cincinnati, Ohio. 
EPA (U.S. Environmental Protection Agency). 1980c. Proscribed procedures for measurement of radioactivity in drinking water, EPA-600/4-80-032. Environmental Monitoring and Support Laboratory, Cincinnati, Ohio.

EPA (U.S. Environmental Protection Agency). 1983. Methods for the chemical analysis water and wastes. EPA 600/4-79-020. Environmental Monitoring and Support Laboratory, Cincinnati, Ohio.

EPA (U.S. Environmental Protection Agency). 1984a. Laboratory Data Validation Functional Guidelines for Evaluating Inorganics Analyses. Office of Emergency and Remedial Response.

EPA (U.S. Environmental Protection Agency). 1984b. Extraction and analysis of priority pollutants in biological tissue, method PPB 12/83. Environmental Services Division, Region IV, Analytical Support Branch, Athens, Georgia.

EPA (U.S. Environmental Protection Agency). 1984c. Hydrological Simulation Program FORTRAN (HSPF), User's Manual for Release 3.0, Region IV, Analytical Support Branch, Athens, Georgia.

EPA (U.S. Environmental Protection Agency). 1986a. Test methods for evaluating solid waste. SW-846, 3d ed. Office of Solid Waste and Emergency Response, Washington D.C.

EPA (U.S. Environmental Protection Agency). 1986b. Statement of work for organic analyses, multi-media, multi-concentration. Contract Laboratory Program, Washington, D.C

EPA (U.S. Environmental Protection Agency). 1987a. Quality criteria for water, May 1, 1987 update, EPA 440/5-86-001, EPA Office of Water Regulations and Standards, Washington, D.C.

EPA (U.S. Environmental Protection Agency). 1987b. Data quality objectives for remedial response activities. EPA/540/G-87/003, OSWER Directive 9355.0-76. Office of Solid Waste and Emergency Response, Washington, D.C.

EPA (U.S. Environmental Protection Agency). 1987c. Statement of work for inorganic analyses, multi-media, multi-concentration. Contract Laboratory Program, Washington, D.C.

EPA (U.S. Environmental Protection Agency). 1988a. Guidance for Conducting Remedial Investigations and Feasibility Studies under CERCLA. OSWER Directive 9335.3-01, Draft. Office of Emergency and Remedial Response, Washington, D.C.

EPA (U.S. Environmental Protection Agency). 1988b. Laboratory Data Validation Functional Guidelines for Evaluating Organics Analyses. Hazardous Site Evaluation Division. 
EPA (U.S. Environmental Protection Agency). 1988c. CERCLA compliance with other laws manual, draft guidance, Vol. 1. OSWER Directive 9234.1-01, EPA Office of Emergency and Remedial Response, Washington, D.C.

Greene, J. A. 1991. Final report on the waste area grouping perimeter groundwater quality monitoring well installation program. ORNL/ER-46. Oak Ridge National Laboratory, Oak Ridge, Tennessee.

Guy, H.P. 1969. Laboratory Theory and Methods for Sediment Analysis. IN: Techniques of Water Resources Investigations of the U.S. Geological Survey. U.S. Geological Survey, Reston, VA.

Guy, H.P., and V.W. Norman. 1982. Field Methods for Measurement of Fluvial Sediment. IN: Techniques of Water Resources Investigations of the U.S. Geological Survey. U.S. Geological Survey, Reston, VA.

Hoffman, F. O., B. G. Blaylock, M. L. Frank, L. Hook, E. L. Etnier, and S. S. Talmage. 1990. Screening of contaminants in the off-site environment. ORNL/ER-9. Oak Ridge National Laboratory, Oak Ridge, Tennessee.

Hook, L. A. et al. 1990. Data base management activities for the Remedial Action Program at ORNL: Calendar year 1989. ORNL/ER-16. Oak Ridge National Laboratory, Oak Ridge, Tennessee.

Huff, D. D., N. D. Farrow, and J. R. Jones. 1982. Hydrologic factors and ${ }^{90}$ Sr transport: A case study. Environ. Geol. 4:53-63.

Jardine, P. M., G. V. Wilson, and R. J. Luxmoore. 1990. Unsaturated solute transport through a forest soil during rain storm events. Geoderma 46:103-118.

Jardine, P. M., and G. K. Jacobs. 1991. Unsaturated transport of inorganic cations in undisturbed soil columns. Proc. Radionuclide Adsorption Workshop, Los Alamos National Laboratory, Los Alamos, New Mexico, September 11-12, 1990.

Kimbrough, C. W., L. W. Long, and L. W. McMahon (eds.). 1990. Environmental Surveillance Procedures Quality Control Program. ESH/Sub/8721706/1. Revision 1. Martin Marietta Energy Systems, Inc., Oak Ridge, Tennessee.

Kroodsma, R. L. 1987. Resource Management Plan for the Oak Ridge Reservation. Vol. 24: Threatened and endangererd animal species. ORNL/ESH-1/24. Oak Ridge National Laboratory, Oak Ridge, Tennessee.

Lal, R. 1988. Soil Erosion Research Methads. Soil and Water Conservation Society, Ankeny, Iowa.

Loar, J. M. (ed.). 1987. First annual report of the ORNL Biological Monitoring and Abatement Program. Draft ORNL/TM. Oak Ridge National Laboratory, Oak Ridge, Tennessee. 
Loar, J. M. (ed.). 1988. Second annual report of the ORINL Biological Monitoring and Abatement Program. Draft ORNL/TM. Oak Ridge National Laboratory, Oak Ridge, Tennessee.

Loar, J. M. (ed.). 1989. Third annual report of the ORNL Biological Monitoring and Abatement Program. Draft ORNL/TM. Oak Ridge National Laboratory, Oak Ridge, Tennessee.

Loar, J. M. (ed.). 1990. Fourth annual report of the ORNL Biological Monitoring and Abatement Program. Draft ORNL/TM Report. Oak Ridge National Laboratory, Oak Ridge, Tennessee.

Loar, J. M. (ed.). 1991. Fifth annual report of the ORNL Biological Monitoring and Abatement Program. Draft ORNL/TM. Oak Ridge National Laboratory, Oak Ridge, Tennessee.

McIntosh, D. L., G. W. Suter II, and F. O. Hoffman. Model of PCB and mercury exposure to mink and great blue heron inhabiting the off-site environment downstream from the U.S. Department of Energy Oak Ridge Reservation. ORNL/ER-xx, Oak Ridge National Laboratory, Oak Ridge, Tennessee. (in press)

Melroy, L. A. and D. D. Huff. 1985. Evaluation of a flow diversion for reducing ${ }^{90} \mathrm{Sr}$ migration from SWSA 4 to Whiteoak Creek. ORNL/TM-9374. Oak Ridge National Labratory, Oak Ridge, Tennessee.

Melroy, I. A., D. D. Huff, and N. D. Farrow. 1986. Characterization of the near-surface radionuclide contamination associated with the bathtub effect at Solid Waste Storage Area 4, Oak Ridge National Laboratory, Tennessee. ORNL/TM-10043. Oak Ridge National Laboratory, Oak Ridge, Tennessee, 45 pp.

Moore, G. K. 1989. Groundwater parameters and flow systems near Oak Ridge National Laboratoly, Tennessee. ORNL/TM-11368. Oak Ridge National Laboratory, Oak Ridge, Tennessee.

Moore, G. K. 1988. Concepts of groundwater occurrence and flow near Oak Ridge National Laboratory. ORNL/TM-10696. Oak Ridge National Laboratory, Oak Ridge, Tennessee.

Oakes, T. W., B. A. Kelly, W. O. Ohnesorge, J. S. Eldridge, J. C. Bird, K. E. Shanks, and F. S. Tsakeres. 1982. Technical background information for the ORNL environmental and safety report, Vol. 4: White Oak Lake and Dam. ORNL/TM-7509/V4. Oak Ridge National Laboratory, Oak Ridge, Tennessee.

ORNL (Oak Ridge National Laboratory). 1988. Quality Assurance Manual. Oak Ridge, Tennessee.

ORNL (Oak Ridge National Laboratory). 1990. Remedial investigation plan for Waste Area Grouping 2 at Oak Ridge National Laboratory, Oak Ridge 'Tennessee. ES/ER-14\&D1. Environmental Restoration Program. Oak Ridge National Laboratory, Oak Ridge, Tennessee. 
Roberson, L. E., and G. M. Logdson. 1989. Environmental Sciences Division Quality Assurance Manual. Oak Ridge National Laboratory, Oak Ridge, Tennessee.

Ross, H. H. 1969. Measurement of $\beta$-emitting nuclides using Cerenkov radiation. Anal. Chem. 41:1260-1265.

Solomon, D. K., J. D. Marsh, I. L. Larsen, D. S. Wickliff, R. B. Clapp. 1991. Transport of contaminants during storms in the White Oak Creek and Melton Branch watersheds. ORNL/TM-11360. Oak Ridge National Laboratory, Oak Ridge, Tennessee.

Spalding, B. P. and T. E. Cerling. 1979. Association of radionuclides with streambed sediments in Whiteoak Creek Watershed. ORNL/TM-6895. Oak Ridge National Laboratory, Oak Ridge, Tennessee.

Spalding, B. P., I. L. Munro. 1984. Determination of the areal distribution of ${ }^{90} \mathrm{Sr}$ in groundwater via single-use boreholes. ORNL/TM-8991. Oak Ridge National Laboratory, Oak Ridge, Tennessee.

Spalding, B. P. and I. L. Munro. 1983. Soda ash treatment of a ${ }^{90} \mathrm{Sr}$-contaminated groundwater seep. J. Environ. Qual. 12(3:366-373.

Suter, G. W. II. 1989. Ecological endpoints. pp. 2-1-2-38 IN W. Warren-Hicks, B. R. Parkhurst, and S. S. Baker, Jr. (eds.), Ecological Assessment of Hazardous Waste Sites: A Field and Laboratory Reference. EPA/660/3-89/013. U.S. Environmental Protection Agency, Corvallis, Oregon.

Stueber, A. M., D. A. Webser, I. L. Munro, N. D. Farrow, and T. G. Scott. 1981. An Investigation of Radionuclide Release From Solid Waste Disposal Area 3, Oak Ridge National Laboratory. ORNL/TM-7323. Oak Ridge National Laboratory, Oak Ridge, Tennessee.

Tardiff, M. F., Information and Integration and Analysis Group, Office of Environmental and Health Protection, ORNL, memo of May 31, 1991. p 48, 44.

Voorhees, L. D., et al. 1988. Annual report of data base management activities for the Remedial Action Program at ORNL: Calendar year 1987. ORNL/TM-10694. Oak Ridge National Laboratory, Oak Ridge, Tennessee.

Voorhees, L. D., et al. 1989. Annual report of data base management activities for the Remedial Action Program at ORNL: Calendar year 1988. ORNL/TM-11147. Oak Ridge National Laboratory, Oak Ridge, Tennessee.

Warren-Hicks. W., B. R. Parkhurst, and S. S. Baker, Jr. (eds.). 1989. Ecological assessment of hazardous waste sites: A field and laboratory reference. EPA/600/3-89/013. U.S. Environmental Protection Agency, Corvallis, Oregon. 


\section{ACKNOWLEDGMENTS}

The authors thank D. D. Huff, J. R. Trabalka, L. A. Hook, B. P. Spalding, G. R. Southworth, F. O. Hoffman, G. Bodenstein, A. L. Brenkert, R. B. Cook, P. A. Schrandt, D. M. Carden, and R. H. Gardner for many helpful comments. We thank J. S. Cox for compiling and prorlucing the document, and the ORNL Environmental Sciences Division graphics group for creatively producing the charts and illustrations. 
APPENDIX A

WAG 2 RI STAFF 
RICHARD W. ARNSETH

$\mathrm{Ph} . \mathrm{D}$., Geological Sciences, 1983, Northwestern University

TOM L ASHWOOD

M.S., Economica, 1975, Murray State University

WIILIAM D. BARTON III

M.S., Civil Engineering, 1980, The Univeraity of Tennessee

F. PAUL BAXTER

Ph.D., Soil Science and Geology, 1970, University of Wisconsin

JOHN J. BEAUCFAMP

Ph.D. Statistics, 1966, Florida State University

B. GORDON BLAYLOCK

Ph.D., Zoology, 1963, The University of Tennessee

DENNIS M. BORDERS

M.S., Environmental Engineering, 1986, The University of Tennessee

HARRY L BOSTON

Ph.D., Botany, 1984, University of Wisconsin-Madison

\section{A. L. BRENKERT}

Doctorandus, Biology, 1968, University of Amsterdam

VENRATESH CHIDAMBARIAH

M.S., Environmental Engineering, 1989, The University of Tennessee

ROGER B CLAPP

Ph.D., Environmental Sciences, 1982, University of Virginia

D. J. DOWNING

Ph.D., Statistics, 1974, University of Florida

\section{E L ETNIER}

M.A., Zoology, 1965, The University of Tenneasee

THOMAS A. FONTANE

Ph.D., Civil and Environmental Engineering, 1989, University of Wisconsin

MARILYN LL FRANR

M.S., Radiation Biology, 1970, The University of Tennessee

ROBERT H. GARDNER

Ph.D., Zoology, 1975, North Carolina State University

\section{F. OWEN HOFFMAN}

Ph.D., Ecology, 1981, The University of Tennessee 
LESLIE A. HOOK

Ph.D., Zoology, 1988, Southern Illinois University

\section{DANIEL JONES}

M.S., Environmental Sciences, 1991, Univensity of Massachussetts

\section{CYNTHIA M KIENDRICK}

B.S., Chemical Engineering, 1984, The University of Tennessee

\section{S. Y. IEEE}

Ph.D., Soil Science, 1973, University of Wisconsin

\section{DAWN E MMILER}

M.S., Biological Science, 1990, University of Minaissippi

\section{GERALD K MOORE}

B.S., Geology and Mineralogy, 1956, Pennsylvania State University

\section{J. E. NYQUIST}

Ph.D., Geophysics, 1986, University of Wisconsin

\section{GEORGE R SOUTHWORTH}

M.S., Aquatic Ecology, 1974, University of Michigan

\section{GLENN W. SUTER II}

Ph.D., Ecology, 1976, University of California, Davis

\section{MARK S. TARDIFF}

M.S., Ecology, 1982, SUNX College of Environmental Science and Forestry

\section{JULIE A WATTS}

M.S., Mathematics/Statistics, 1968, Tennessee Technological University

\section{S. WICKLIFI}

M.S., Geology, 1988, Univerrity of Arkansas

\section{TOMAY WRIGHT}

Ph.D., Statistics, 19m, Ohio State University 

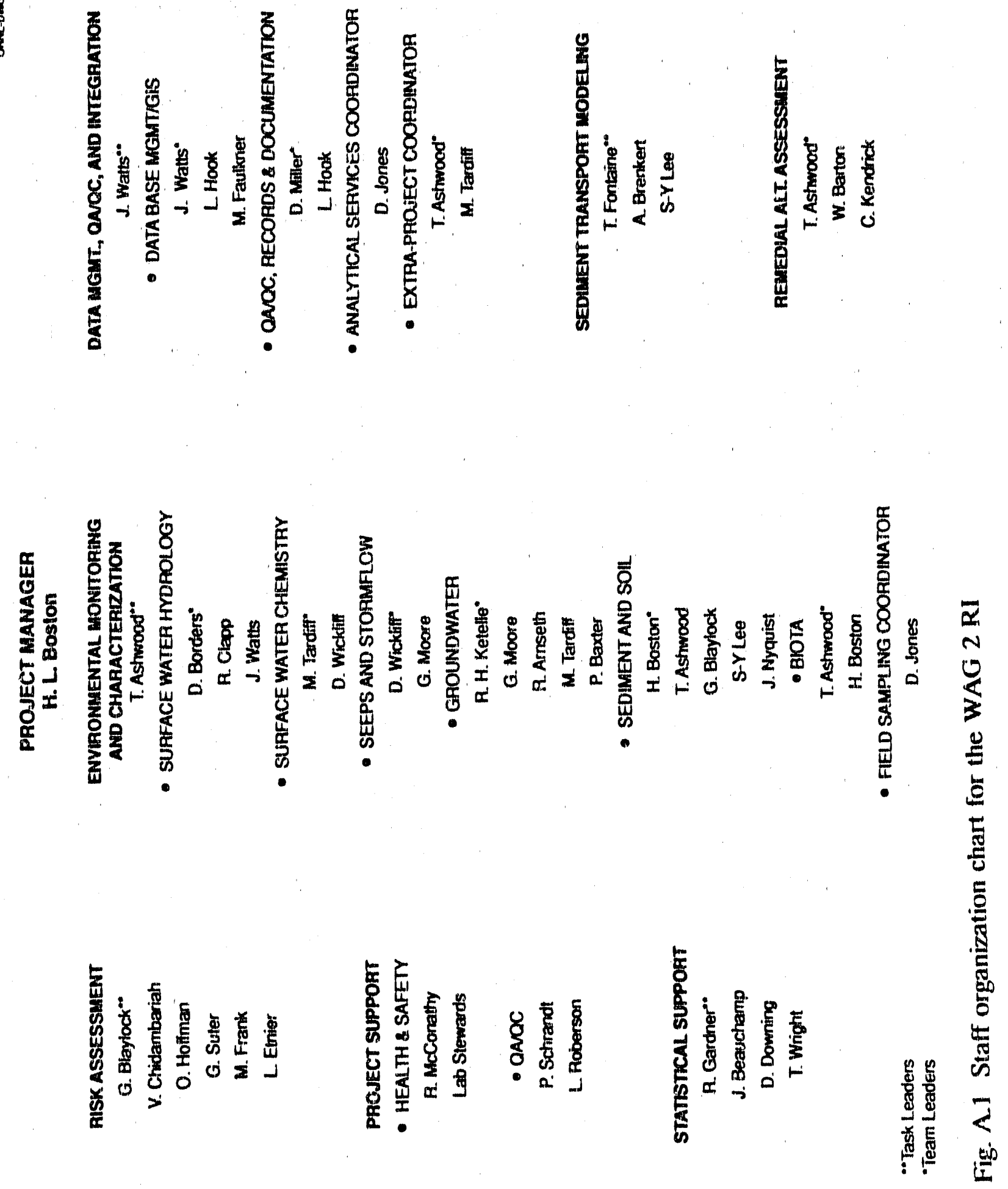


\section{APPENDIX B \\ TYPE OF DATA REQUIRED FOR HSPF MODEL OF SEDIMENT TRANSPORT}




\section{Appendix B}

\section{TYPE OF DATA REQUIRED FOR HSPF MODEL, OF SEDIMENT TRANSPORT}

\section{Note: No distinction is made between data required to set parameters or to calibrate parametere.}

\section{RAINFALLRUNOFF PROCESSES (STREAMFLOW GENERATION)}

precipitation (10-min intervals if possible)

potential evapotranspiration

stream discharge hydrographs

initial conditions for moisture storage in upper and lower soil zones and in intertlow and active groundwater

density and type of vegetative cover (hillslope and riparian)

root zone depth

land use (including retention and detention storage in impervious areas)

soil type or permeability, soil temperature

catchment area, lengths and slopes of channels and hillslopes

roughness coefficients for hillstopes and channels (Manning's $n$ )

groundwater levels, hydraulic conductivity of deep groundwater systems

depth-volume-surface area relations for channels and lakes

backwater profiles for specific discharges (c.g., HEC-2 output)

specific weight of stream water

ratio of maximum stream velocity to average stream velocity

\section{SEDIMENT SUPPLY (EROSION, SCOUR, AND DEPOSITION)}

rainfall intensity, overland flow and initial conditions for surface flow storage (this information is provided from the hydrologic components in HSPF)

areas with erosion resistant cover (e.g., vegetation, mulch, and erosion protection)

supporting soil management practice (the equivalent of agricultural soil management practices that apply to unprotected soil in WOC)

factors for the susceptibility for soil detachment and soil matrix scour

solids transport capacity of flow over impervious surfaces 


\section{SEDIMENT TRANSPORT DURING STORMS}

sediment-discharge rating curves developed from measurements of suspended and bedlond during stormflow

percentage by weight of gravel, sand, silt, and clay fractions

settling velocities of each size fraction

particle density

critical shear stress for deposition and scour of silt and clay

erodability coefficient of silt and clay

median and $60 \%$ grain diameter

initial storage of grvel, sand, silt, and clay in streambed

water temperature

hydraulic radius of each channel segment

\section{SEDRMENT.CONTAMINANT RELATIONSHIPS}

potency fretors: monthly values of the concentration of a contaminant attached to sediment that has been eroded from a pervious or impervious surface (generally a function of the distribution coefficient ( $K_{d}$ for the contaminant and the grain size and mineralogy of the sediment particle)

supply of each contaminant to the system (time series of flux entering WOC either adsorbed to sediment or in dissolved phase)

susceptibility of a contaminant to wash-off by overland flow

concentration of contaminants in subsurface flow (interflow and groundwater flow)

transfer rates for adsorption/desorption of contaminants on sediment

data for heat exchange and water temperature (solar radiation, cloud cover, air temperature, dewpoint temperature, and wind speed)

decay rate (and temperature correction coefficient) for decay of an adsorbed contaminant 


\section{APPENDIX C}

\section{WAG 2 PROCEDURES}

Laboratory Procedures Manual for the ORAU Environmental Survey and Site Assessment Program . . . . . . . . . . . . . . . . . . . 3

Strontium-90 Determinations by Cerenkov Radiation Counting for Well Monitoring at Oak Ridge National Laboratory, ORNL/TM-7760 
C-3

LABORATORY PROCEDURES MANUAL

FOR THE

ENVIRONMENTAL SURVEY AND SITE ASSESSMENT PROGRAM

\author{
Prepared by: \\ C.F. Weaver, M.J. Laudeman \\ and \\ S. Shanmugan
}

Oak Ridge Associated Universities

Oak Ridge, Tennessee 37831-0117

Revision 6

April 1991 


\section{SECTION 1}

\section{INTRODUCTION}

The Environmental Survey and Site Assessment Program of Oak Ridge Associated Universities conducts environmental survey activities for the Department of Energy under contract DE-ACO5-760R00033 and for the Nuclear Regulatory Commission under several interagency agreements.

Sites surveyed under this program are primarily those where residual contamination from previous operations may pose a potential risk to the environment of the site or the health and safety of those presently occupying the site. Other activities include monitoring of radioactive effluents from currently operating facilities and miscellaneous technical assistance to the funding agencies.

The purpose of this Procedures Manual is to provide a standardized set of procedures that document the analytical activities of the program in an auditable manner. These procedures are applicable to both the DOE and NRC operations. Procedures presented here are limited to those associated with laboratory and analytical operations; procedures related to field survey activities are presented in a separate document.

This manual was prepared by C.F. Weaver, Laboratory Manager for the Program, with the assistance of laboratory supervisors $M$. J. Laudernan and S. Shanmugan. Other staff members (previous and present) contributed to this manual. with assistance, advice, and support.

Laboratory Procedures Manual

ORAU/ESSAP

Approved:
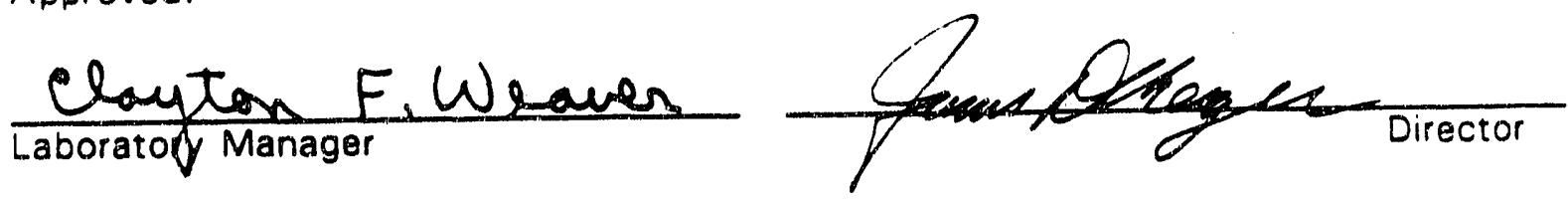

Revision No. 6

Date: April 2, 1991

Page 1 of Section 1 


\section{C-5}

SECTION $13(2)$

DETERMINATION OF STRONTIUM-90 AND .89 IN SOIL AND SEDIMENT

\section{PART A}

Principle

The soil is dissolved using a pyrosulfate fusion which removes silicates and converts the remaining material to a water soluble form. Strontium is carried on strontium and lead sulfate to minimize the amount of strontium carrier. The strontium sulfate is dissolved in additional EDTA, and ferric and other insoluble hydroxides are precipitated at a pH of 12 to 14. The strontium is separated from residual calcium by reprecipitating strontium sulfate from EDTA at a DH of 4.0. DTPA is used to effect separation of barium as the chromate from strontium. Strontium sulfate is metathesized to the carbonate prior to the chromate step to rid the system of interfering sulfate ion. The final precipitate is strontium carbonate which is counted on a low-background beta counter.

\section{REFERENCES}

1. C.W. Sill, K.W. Puphol and F.D. Hindman, Anal. Chem, 46, 1725 (1974)

2. Don B. Martin, Anal. Chem, 51, 1968 (1979)
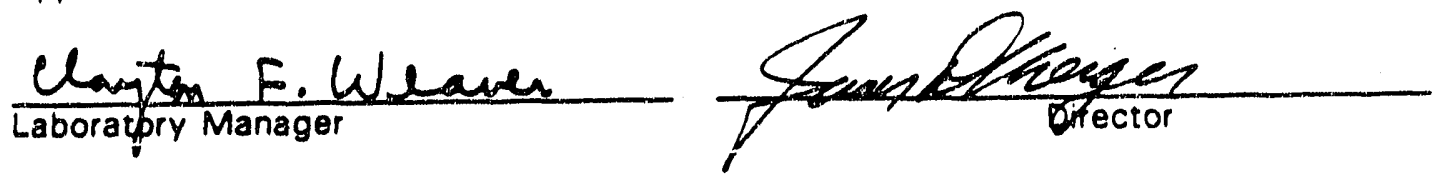
SECTION $13(2)$

\section{STRONTIUM-90 AND -89 IN SOIL AND SEDIMENT}

\section{Part B}

1.0 This is a radiochemical procedure for the determination of strontium-90 in soil at environmental levels.

\subsection{REAGENTS}

2.1 Acetic Aćid $\left(\mathrm{HC}_{2} \mathrm{H}_{3} \mathrm{O}_{2}\right)$ : Glacial, $17.4 \mathrm{~N}$

2.2 Acetic Acid, 6M: To $500 \mathrm{ml}$ reagent water, add $342 \mathrm{ml}$ Glacial Acetic Acid. nilute to 1 liter.

2.3 Barium Chloride Solution, $0.9 \%$ : Dissolve $9 \mathrm{~g} \mathrm{BaCl}_{2} 2 \mathrm{H}_{2} \mathrm{O}$ in $900 \mathrm{ml}$ reagent water. Dilute to 1 liter.

2.4 DTPA, $0.2 \mathrm{M}$ : Dissolve $39 \mathrm{~g}$ DTPA and $25 \mathrm{~g} \mathrm{NaOH}$ in $400 \mathrm{ml}$ reagent water. Vacuum filter through an HA 0.45 micron filter. Dilute $10500 \mathrm{ml}$.

2.5 EDTA, 0.6M: To $800 \mathrm{ml}$ reagent water, slowly add $223 \mathrm{~g}$ Disodium Ethylene diamine tetraacetate while stirring. Heat gently and slowly add $\mathrm{NaOH}$ until the EDTA dissolves. Vacuum filter through a 0.45 micron HA filter. Dilute to 1 liter with reagent water. Alternate: Replace $223 \mathrm{~g} \mathrm{Na}{ }_{2}$ EDTA with $194 \mathrm{~g} \mathrm{H}$.EDTA and $48 \mathrm{~g} \mathrm{NaOH}$.

2.6 Ethanol 95\%: May be available as such or dilute $475 \mathrm{ml}$ Ethanol to $500 \mathrm{ml}$ and place in a squirt bottle.

2.7 Sodium hydroxide, 10M

2.7a Hydrochloric Acid, $92 \mathrm{M}$, concentrated

2.8 Hydrochloric Acid, 6M: Measure $500 \mathrm{ml}$ reagent water in a 1 liter graduated cylinder. Add 12M HCl to 1 liter.

2.9 Hydrogen Peroxide, $30 \%\left(\mathrm{H}_{2} \mathrm{O}_{2}\right)$

2.10 Lead Nitrate Solution. 1.6\%: Dissolve $16 \mathrm{~g} \mathrm{~Pb}\left(\mathrm{NO}_{3}\right)_{2}$ in $800 \mathrm{ml} \mathrm{reagent} \mathrm{water.}$ Dilute to 1 liter.

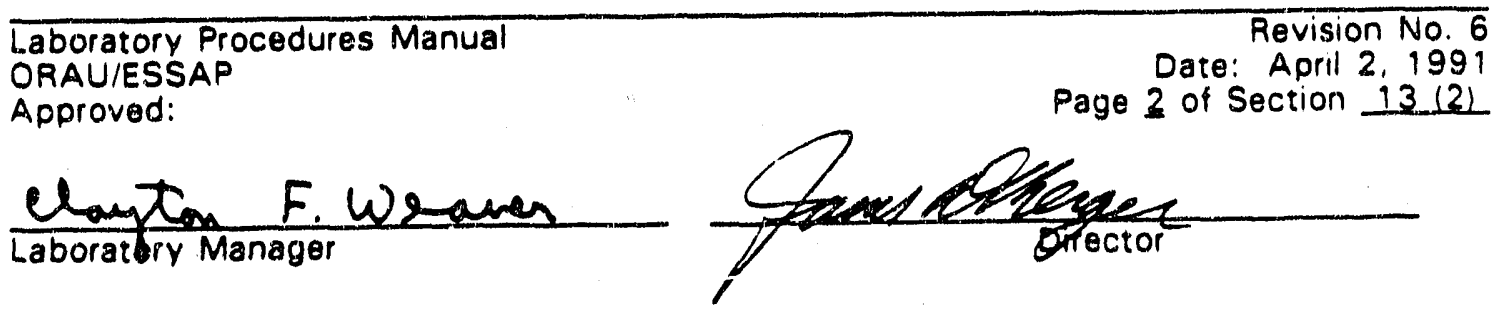


2.11 Metacresol Purple, indicator: Dissolve $0.1 \mathrm{~g} \mathrm{MCP}$ in $26.2 \mathrm{ml} 0.01 \mathrm{~N} \mathrm{NaOH}$. Dilute to $250 \mathrm{ml}$.

2.12 Phenolphthalein, indicator: Dissolve $0.05 \mathrm{~g}$ phenolphthalein in $50 \mathrm{ml}$ ethanol. Dilute to $100 \mathrm{ml}$ with "water.

2.13 Sodium Acetate/Acetic Acid Solution: Dissolve $50 \mathrm{~g} \mathrm{Na} \mathrm{C} \mathrm{H}_{3} \mathrm{O}_{2}$ in $800 \mathrm{ml}$ reagent water. Add $100 \mathrm{ml}$ glacial acetic acid. Dilute to 1 liter.

2.14 Sodium Carbonate, $10 \%$ : Dissolve $1.00 . \mathrm{g} \mathrm{Na}_{2} \mathrm{CO}$, in $800 \mathrm{ml}$ reagent water. Dilute to 1 liter.

2.15 Sodium Chrornate, 1.0M: Dissolve $162 \mathrm{~g} \mathrm{Na} \mathrm{CrO}_{4}$ in $800 \mathrm{ml}$ reagent water. Dilute to 1 liter.

2.16 Sodium Hydroxide, 10M: Dissolve $200 \mathrm{~g} \mathrm{NaOH}$ in $200 \mathrm{ml}$ reagent water. Add the NaOH slowly; cool in an ice bath as needed. Dillute to $500 \mathrm{ml}$.

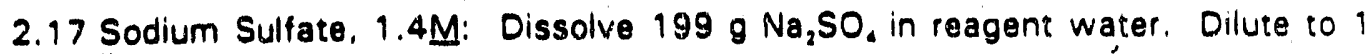
liter.

2.18 Strontium Carrier, $40 \mathrm{mg} / \mathrm{ml}$ : Refer to step 2.32 of section $13(1)$ for preparation. Refer to step 4.5 of section $13(1)$ for standardization.

\subsection{APPARATUS}

3.1 Platinum dish

3.2 Watch glass

3.3 Hot plate

3.4 Beaker

3.5 Centrifuge tube, $40 \mathrm{ml}$

3.6 Vortex mixer

3.7 Centrifuge

3.8 $\mathrm{pH}$ meter

3.9 Low-background beta counter

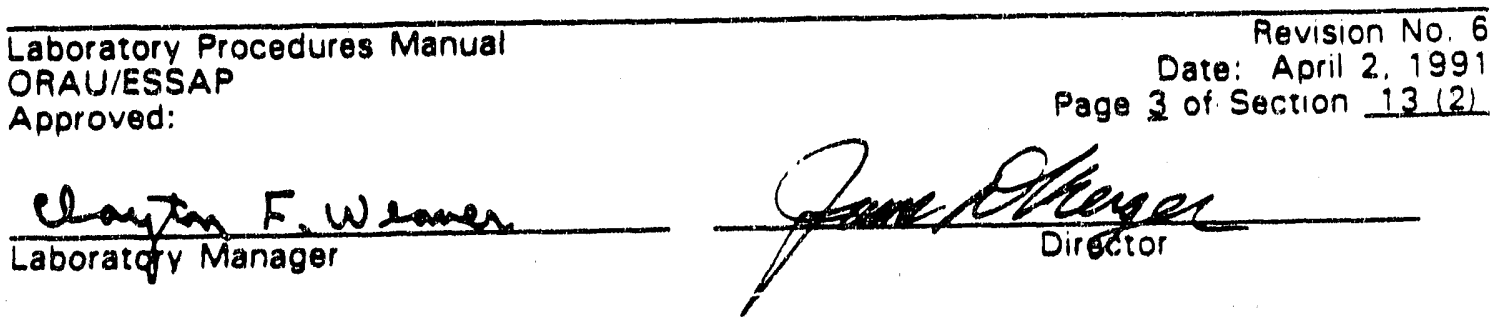




\subsection{PROCEDURE}

4.1. Weigh 5.0 $\mathrm{g}$ of the -35 mesh soil into a dry semiflat bottomed platinum dish.

4.2 Add $3.0 \mathrm{ml}$ of concentrated HNO, a few drops at a time as fast as the reaction will permit.

4.3 Heat gently until the reaction has subsided and the soil remains just moist.

4.4 Add an appropriate amount of the tracers (usually $1.0 \mathrm{ml}$ ). Record the theoretical yield on the data sheet.

4.5 Add $15 \mathrm{~g}$ of anhydros KF to the dish. Slowly add $15.20 \mathrm{ml} 48 \%$ HF to the soll and $K F$ in the platinum dish.

4.6 Hold the dish over a biast burner. Heat gently and agitate to mix into a thickening slurry. Heat as quickly as possible to dryness.

4.7 Place the platinum dish on a ring stand using a nichrome triangle.

4.8 Use a high temperature blast burner and apply as much heat as possible, to bring the temperature to about $900^{\circ} \mathrm{C}$. This should take about 4 minutes. The total dissolution process will take another 2 minutes. Swirl the hot melt to ensure removal of sample clinging to the sides of the dish.

4.9 Remove the melt from the burner and swirl gently around the dish to form a thin layer upon cooling. (Never sit hot platinum on iron).

4.10 After the cake has cooled to room temperature, add a $15.20 \mathrm{ml}$ of concentrated $\mathrm{H}_{2} \mathrm{SO}$, to the fluoride cake. The acid should be added to the edge of the cake and allowed to run to the bottom of the dish. Because of the evolution of SiF." this step muss be done in a hood.

4.11 After the addition of the $\mathrm{H}_{2} \mathrm{SO}_{\text {, }}$ heat as fast as frothing will allow until the fluoride cake is totally dissolved and a thick slurry forms.

'For environmental samples, a 5 or $10 \mathrm{~g}$ aliquot may be used. The quantity of reagents used must be increased proportionally, except, for HNO,. This comment applies only through the pyrosulfate fusion.

Laboratory Procedures Manual

Revision No. 6

ORAUIESSAP

Approved:

Date: April 2, 1991
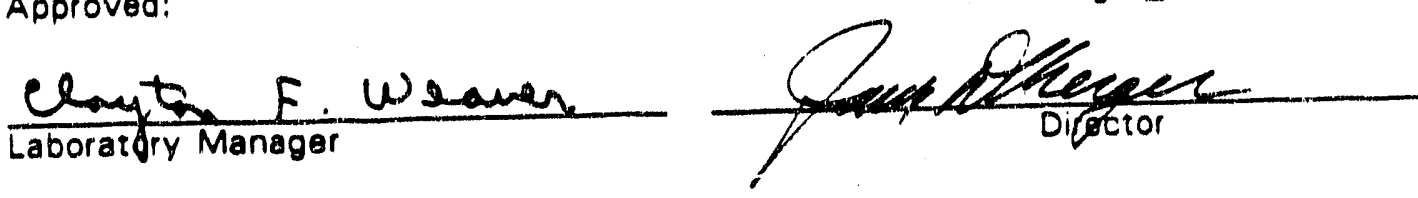
4.12 Remove from the heat and add $7.0 \mathrm{~g}$ of anhydrous $\mathrm{Na}_{2} \mathrm{SO}_{4}$ to the slurry. Using a hot plate, gently heat the sample until the $\mathrm{Na}_{2} \mathrm{SO}$, dissolves. Place the sample over the blast burner with small flame and heat until the slurry begins to turn a golden brown. Slowly increase the temperature until the slurpy is completely melted, then maintain this temperature for approximately 1 minute.

4.13 To a 1 liter or $800 \mathrm{ml}$ beaker, add $350 \mathrm{ml}$ of water and a teflon stir bar. Cover and heat to boiling. Add $7 \mathrm{ml} 12 \mathrm{M} \mathrm{HCl}$ and the pyrosulfate cake. Boil for 10 minutes.

4.14 While stirring add $1 \mathrm{ml}$ of $30 \% \mathrm{H}_{2} \mathrm{O}_{2}$ followed immediately by siowly adding 10 $\mathrm{ml}$ of $1.6 \% \mathrm{~Pb}\left(\mathrm{NO}_{3}\right)_{2}$ and boil for one minute. Repeat the lead addition two more times.

$4.15 \mathrm{Cool}$ beaker in a cold water bath for 15 minutes. After which, add another 10 $\mathrm{ml}$ portion of $\mathrm{Pb}\left(\mathrm{NO}_{\mathrm{g}}\right)_{2}$ slowly while stirring. If possible, allow the precipitate to stand overnight.

4.16 Decant or siphon as much liquid as possible without disturbing the precipitate. Then transfer the precipitate to centrifuge tube. Centrifuge and discard the supernate.

4.17 Wash the precipitate with $25 \mathrm{ml}$ of $5 \% \mathrm{~K}_{2} \mathrm{SO}$, containing $4 \mathrm{mi}$ sulfuric acid per liter of wash. Centrifuge and discard the supernate.

4.18 Loosen the precipitate on a vortex mixer. Add $5 \mathrm{ml}$ reagent water, a drop of metacresol purple, and mix. Add 1OM NaOH dropwise, while stirring, to the purple endpoint. Add $10 \mathrm{ml}$ of $0.6 \mathrm{M}$ EDTA, mix, and heat in a water bath until the precipitate dissolves. Do not discard any undissolved white precipitate.

4.19 Add $5 \mathrm{ml}$ of $1.4 \mathrm{M} \mathrm{Na} \mathrm{SO}_{4}$, glacial acetic dropwise to the yellow end point of the MCP indicator, and then $5 \mathrm{ml}$ of $6 \mathrm{M}$ acetic acid to precipitate strontium sulfate. The pH of the solution should be 4.0. Heat until the precipitate settles Centrifuge and discard the supernate.

4.20 At this point, continue the procedure with one sample at a time. Throughly loosen the precipitate on a vortex mixer. Metathesize the strontium carbonate by stirring and heating the precipitate in $20 \mathrm{ml}$ of $10 \%$ sodium carbonate for 10 minutes centrifuge and discard the supernate.

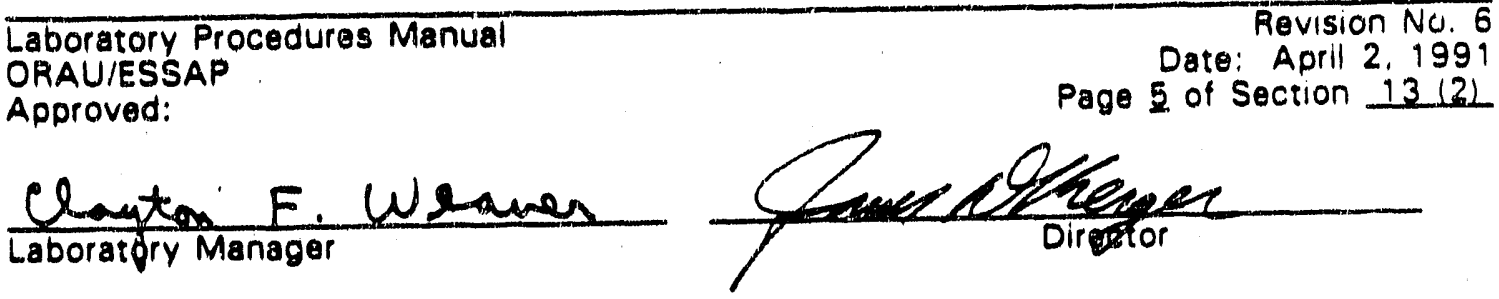


4.21 Add $2 \mathrm{ml}$ of $6 \mathrm{M} \mathrm{HCl}$ to the strontium carbonate precipitate, and after the effervescence stops add $5 \mathrm{ml}$ of $0.2 \mathrm{M}$ DTPA. Add 1 drop of phenolphthalein indleator and $10 \mathrm{M} \mathrm{NaOH}$ dropwise to the red endpoint.

4.22 Add $1 \mathrm{ml}$ of $0.9 \%$ barium chioride dehydrate solutian and $5 \mathrm{ml}$ of $1.0 \mathrm{M}$ sodium chromate and heat the solution for 2 minutes. Add 1 drop of phenophthalein. glacial acetic acid dropwise with stirring to the yellow of the chromate ion, and then add $1 \mathrm{ml}$ of $6 \mathrm{M}$ acetic acid. Heat and cool the solution for 2 minutes each. Centrifuge 5 minutes and discard the vellow chromate precipitate.

4.23 Do not proceed beyond this point unless the procedure can be completed.

4.24 Add $10 \mathrm{mi}$ of $1.4 \mathrm{M} \mathrm{Na} 50$, and $2 \mathrm{ml}$ of glacial aceilc acid to the supernate to procipitato strontium sulfate. The ph should be 4.5 RECORD THE TIME AS THE START OF INGROWTH OF Y9O (elution tima). Heat the solution for 5 minutes and cool for 10 minutes. Centrifuge and discard the supernate.

4.25 Throughiy loosen the precipitate on a vortex mixer. Metathesize the strontium sulfate precipitate to strontium carbonate by adding $20 \mathrm{ml}$ of $10 \%$ sodium carbonate while hesting and stirring for 10 minutes, Cool for 2 minutes.

4.26 Filter the carbonate precipitate on a tared DM-450 filter paper. Wash with reagent water and $95 \%$ ethanol. Record filter time. Air dry, wait 6 hours, and count.

4.27 Rofer to Saction $13(1)$ page $12,5.0$.

4.28 Refer to Section 13(1) page 10 for instrument calibration instructions.

Laboratory Procedures Manual

ORAU/ESSAP

Approved:

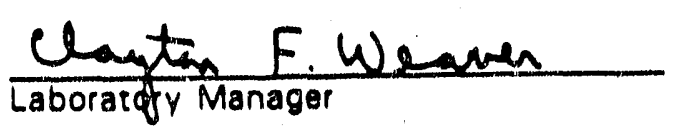

Revision No. 6

Date: April 2.1991

Page 6 of Section $13(21$ 
C-11

\title{
SECTION 14
}

\section{DETERMINATION OF TECHNETIUM-99 IN SOIL AND WATER}

\author{
Part A
}

Principle

The soil samples are leached with sodium hypochlorite and hydrogen peroxide. The leachates are extracted with methyl ethyl ketone, dissolved in water, scavenged by hydroxide precipitation, and recycled through the methyl ethyl ketons extraction. The final water strip is dried on stainless steel planchets and counted on a low-background beta counter. Water samples are treated as leachates and carried through the same procedure.

\section{REEERENCE}

1. F.O. Hoffman et al. Samoline of technetlum-99 in vegeration and soils in the vicinity of eoergine oaseous diffusion olants. ORNL/TM-7386.

Laboratory Procedures Manual
ORAU/ESSAP
Approved:
Canty F.Whawen

Laboratory Manager
Revision No. 6

Date: April 2, 1991

Page 1 of Section 14 
Part B

\subsection{PUAPOSE AND SCOPE}

This is a radiochomical procedure for the determination of technetium-99 in sediment and soll at environmental levels.

\subsection{REAGENTS}

2.1 Sodium hypochlorite, $6 \% \mathrm{NaOC} 1$.

2.2 Hydrogen peroxide, $30 \% \mathrm{H}_{2} \mathrm{O}_{2}$.

2.3 Ammonium hydroxide, Cone. $\mathrm{NH}_{4} \mathrm{OH}$.

2.4 Sodium hydroxide, $50 \% \mathrm{w} / \mathrm{w} \mathrm{NaOH}$.

2.5 Methyl ethyl katone (MEK).

2.6 Technetium-99, standardized solution.

\subsection{APPARATUS}

\subsection{Balance}

\subsection{Centrifuge}

\subsection{Labware}

3.3.1 Beakers, 600-ml tall-form, 250-ml, and $100 \cdot \mathrm{ml}$.

3.3.2 Centrifuge tubes and bottles, $50 . \mathrm{ml}$ and $250 \cdot \mathrm{ml}$ glass.

3.3.3 Separatory funnels, 600 , and $125 \cdot \mathrm{ml}$.

3.4 Pasteur pipets

3.5 Hot plate

3.6 Low-background beta counter.

3.7 Stainiess steel planchets, two inch diameter.

Laboratory Procedures Manual OAAUIESSAP

Approved:

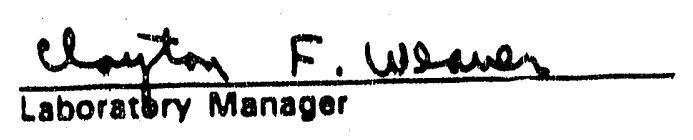

Revision No. 6 Date: April 2, 1991 Page 2 of Section 14 
4.0 PROCEDURE EOR SAMPLE PAEPARATION FOR DETERMINATION OF IECHNETIUM-99

4.1. If the sample is water, take a $300 \mathrm{ml}$ aliquot in a $600 \mathrm{ml}$ beaker and add $15 \mathrm{~g}$ of $\mathrm{NaOH}$. Go to 4.7 and continue. if the sample is soil transter $10 \mathrm{~g}$ of the test soll that has been dried, milled, and sleved to a $250 \mathrm{ml}$-tall beaker and slowly add $150 \mathrm{ml}$ of $6 \% \mathrm{NaOCl}$ (Clorox).

4.2 Digest at $80^{\circ} \mathrm{C}$ to $90^{\circ} \mathrm{C}$ for 1 hour while stirring by magrietic stirring bar.

4.3 Transfer the sample to $250 \mathrm{ml}$ centrifuge bottle and oentrifuge at 3300 RPM for 10 minutes.

4.4 Decant the supernate into a $600 \mathrm{ml}$ beaker.

4.5 Add $150 \mathrm{ml}$ of $\mathrm{NaOCl}$ to the $250 \mathrm{ml}$ centrifuge bottle and stir with a teflon rod to resuspend the soil.

4.6 Centrifuge at 3300 RPM for 10 minutes and add the supernatant to the first leached solution in the $600 \mathrm{ml}$ beaker, then discard the remaining soll into a radlouctive waste container.

4.7 Add $30 \% \mathrm{H}_{2} \mathrm{O}$, to the solution in $1 \mathrm{ml}$ increments and heat to boiling (care must be taken at this point because there is an increased tendency to boil over) until the solution clears, and the volume is reduced to approximately. $75 \mathrm{ml}$. At this point there will be considerable crystallization.

4.8 Cool and transfer by filtering through Geiman filter poper 10.45 micron) into a $250 \mathrm{ml}$ graduated cylinder then dilute to the $200 \mathrm{ml}$ mark with reagent water.

4.9 Transfer to $600 \mathrm{ml}$ baaker and adjust the $\mathrm{pH}$ to 14.0 with $50 \%$ sodium hydroxide (NaOH) using $\mathrm{pH}$ paper. It will take approximately $30 \mathrm{ml}$ of the $50 \%$ $\mathrm{NaOH}$ for a total of $230 \mathrm{ml}$.

4.10 Add an equal volume of methyl ethyl ketone (MEK). Transfer the solution to a $600 \mathrm{ml}$ soparatory funnel.

4.11 Extract, using a mechanical shaker for 10 minutes. Drain the aqueous phase into another separatory funnel and save the MEK.

Laboratory Procodures Manual
ORAU/ESSAP
Approved:

Revision No. 6

Approvod:

Date: April 2, 1991
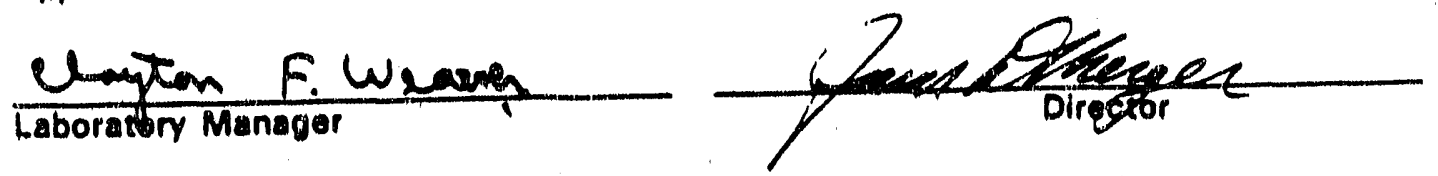
4.12 Add an equal volume of MEK to the aqueous phase and extract for 10 minutes on the shaker.

4.13 Discard the aqueous phase into radioactive waste container.

4.14 Combine the two MEK phases into a $600 \mathrm{ml}$ beaker, add $5 \mathrm{ml}$ reagent water and let thio MEK evaporate over night in a fume hood.

4.15 Transfer the $5 \mathrm{ml}$ that remains in the beaker to a $50 \mathrm{ml}$ centrifuge tube.

4.16 Add 4 drops of conc. hydrochloric acid (HC1) and 10 drops of $30 \%$ hydrogen peroxide $\left(\mathrm{H}_{2} \mathrm{O}_{2}\right)$. Rinse the beaker with $5 \mathrm{ml}$ reagent water and add to the centrifuge tube then add 15 drops of $0.9 \mathrm{M}$ Fo(NO,), ferric nitrate solution, mix well then add $3 \mathrm{ml}$ of $50 \% \mathrm{NaOH}$ and stir well, let stand 30 minutes.

4.17 Centrifuge at 3300 RPM for 10 minutes and transfer the supernatant to a 125 $\mathrm{mi}$ separatory funnol.

4.18 Wash the precipitate with $5 \mathrm{ml}$ of reagent water. Centrifuge at 3300 RPM for 10 minutes and add the supernat int to the separatory funnel.

4.19 Add an equal volume of MEK and extract on mechanical shaker for 10 minutes.

4.20 Drain the aqueous phase into a clean $125 \mathrm{ml}$ separatory tunnel and extract with an equal volume of MEK for 10 minutes.

4.21 Combine the MEK solutions into a $100 \mathrm{ml}$ beaker. Add $1 \mathrm{ml}$ reagent water and allow the MEK to ovaporate in a fume hood. It will take at least 1.2 hours.

4.22 Transfer the approximately $1.0 \mathrm{ml}$ of water to a tared planchet, rinse the beaker two simes with $1.0 \mathrm{ml}$ portions of reagent water and add to the planchet, let dry under infrared lamp.

4.23 Count in a low-background beta counter for at least 30 minutes to determine the technotium 99 activity.

\subsection{CALCULATIONS}

5.1 The data from this procedure are calculated as gross beta except that the system is calibrated using on NIST traceable Standard TC.99. A BASIC prograrn TC99 is usod.

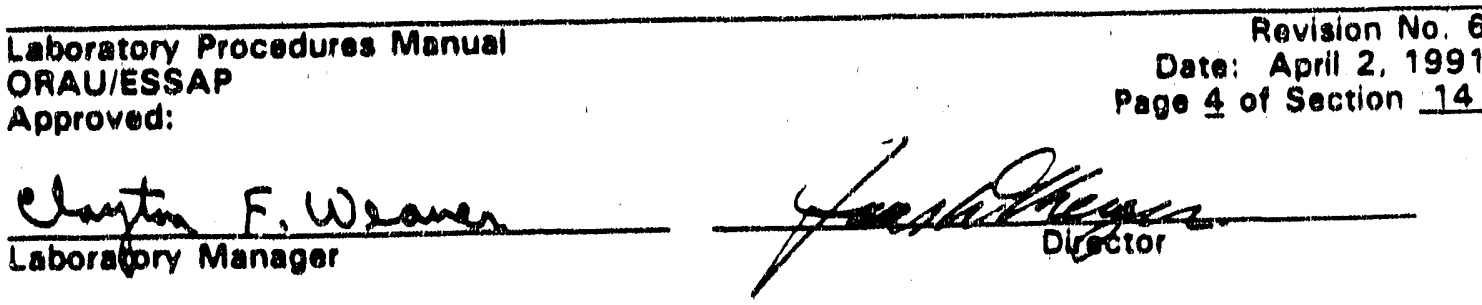


GROSS ALPHA AND BETA CALCULATION SHEET

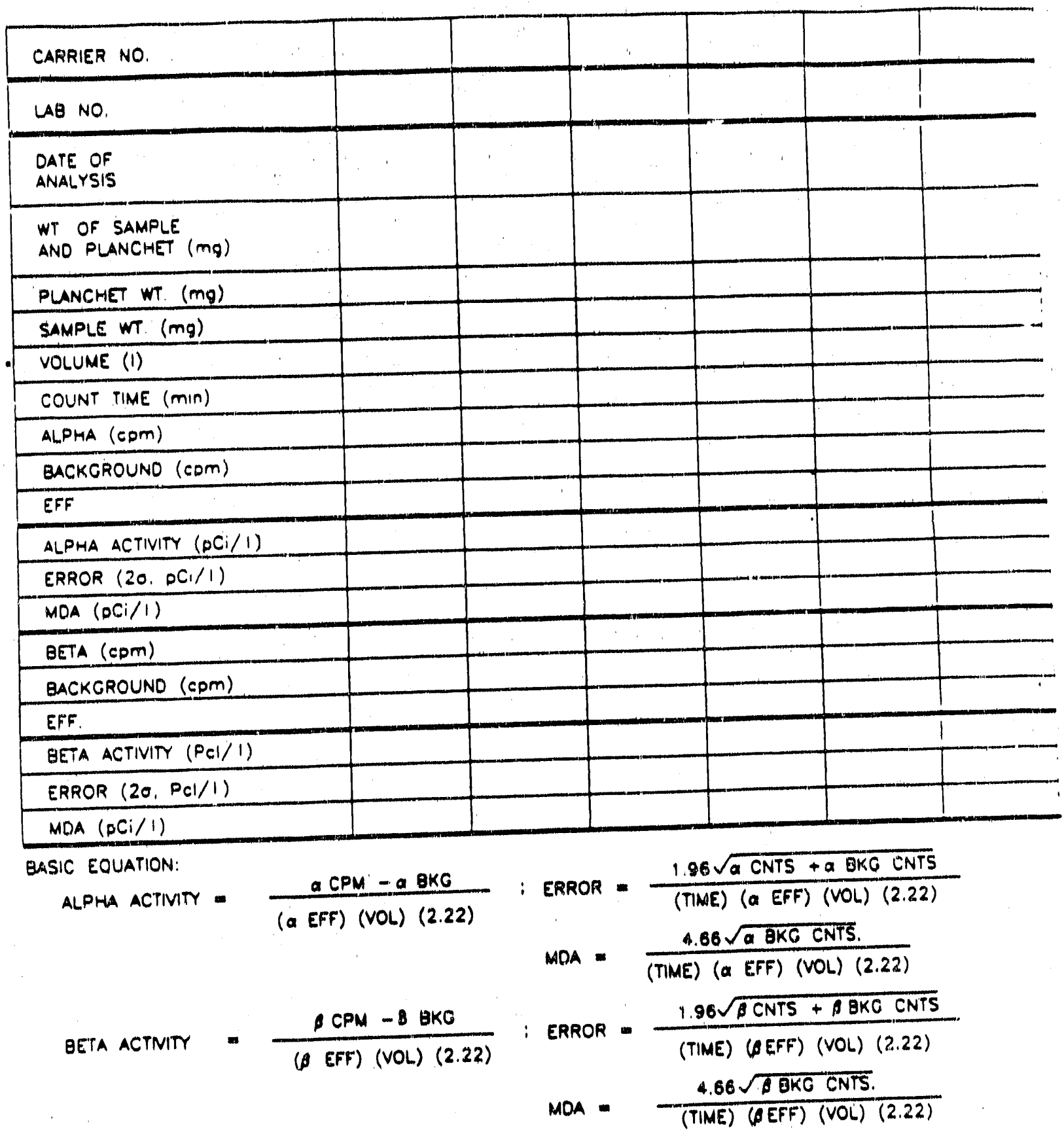

EFFICIENCY INCLUDES CORRECTION FOR SELF ABSORPTION IF NECESSARY.

- USE VOLUHA (L) OR DRY WEIGHT (G) FOR ALL CALCULATIONS UNLESS DIRECTED OTHERWISE.

BATCM NO.

ANALYS̄T̃

REVIEWED GY

DATE 


\section{SECTION 95}

ISOTOPIC DETERMINATION OF AMERICIUM, PLUTONIUM, URANIUM, AND THORIUM IN SOIL, WATER, AIR FILTERS. AND BIOTIC MATERIAL

Part A

Principle

Samples of soil are dissolved by a combination of potassium fluoride and pyrosulfate fusions. The cake is dissolved and all alpha emitters are precipitated by carrying with barium sultate. The barium sulfate is dissolved and the uranium, thorium, and plutonium are separated by liquid-liquid extraction. They are then precipitated by carrying with cerium fluoride and counted with an alpha spectrometer system.

Samples of water are acidified and evaporated to dryness. The residue is then treated as soil.

Samples of air filters and biotic material are ashed. The residue is then treated as soil.

\section{REFERENCES}

1. Claude W. Sill, Kenneth Puphal, and Forest D. Hindman, Anal. Chem. 46, 1725 (1974).

2. Claude W. Sill and Roger L. Williams, Anal. Chem. 53, 412 (1981).

3. Claude W. Sill, Anal. Chem. 46, 1426 (1974).

4. Claude W. Sill, Anal, Chem, 36, 675 (1964).

5. Roger P. Bernabee. Donald R. Percival, and Forest D. Hindman, Anal Chem. 52. 235 ! (1980).
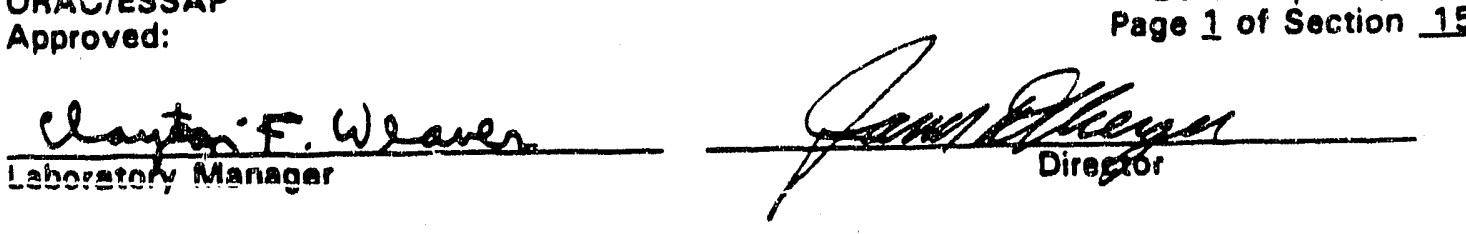
Part B

1.0 This is a radiochemical procedure for the determination of Americium. Plutonium, Uranium, and Thorium isotopes in environmental samples.

\subsection{PROCEDURE}

2.1 Obtain copies of references 1 through 5 from the laboratory supervisor. Equipment lists are found in these documents. All procedures require gloves. lab coats and eye protection at all times.

\subsection{Reagents}

\subsubsection{Aliquot-336 (NOs). 30\%}

Dissolve $900 \mathrm{ml}$ of aliquot-336 (Cl) in $2100 \mathrm{ml}$ of xylene in a 5 liter separatory funnel. Shake vigorously for 4 minutes with each of two successive $500 \mathrm{ml}$ portions of $4 \mathrm{M}$ nitric acid $\left(\mathrm{HNO}_{3}\right)$ to convert the amine to the nitrate form. Wash the organic solution three successive times with 1 liter of reagent water, again shaking for 4 minutes each time. Draw the organic phase into a suitable storage bottle.

2.2.2 Aluminum Nitrate, 2.2N:

Weigh $841 \mathrm{~g}$ of dry $\mathrm{Al}\left(\mathrm{NO}_{3}\right)_{3}, 9 \mathrm{H}_{2} \mathrm{O}$ into a 2 liter beaker. Add $300 \mathrm{ml}$ of reagent water and warm until the salt dissolves. Add $85 \mathrm{ml}$ of conc. HNO,. Vacuum filter through a Dm-450 membrane filter mounted in a glass chimney. Dilute and cool the solution to give a final volume of 1 liter, the density should be $1.370 \mathrm{~g} / \mathrm{ml}$ at $25^{\circ} \mathrm{C}$. If the density is low, multiply the difference between the measured density and 1.370 by 4400 and add this amount of solid. If the density is high, add a small amount of water, stir and recheck.

\subsubsection{Barium Chloride, $0.45 \%$ :}

Dissolve $9.0 \mathrm{~g} \mathrm{BaCl}_{2} 2 \mathrm{H}_{2} \mathrm{O}$ in $1900 \mathrm{ml}$ of reagent water. Dilute to 2 liters.

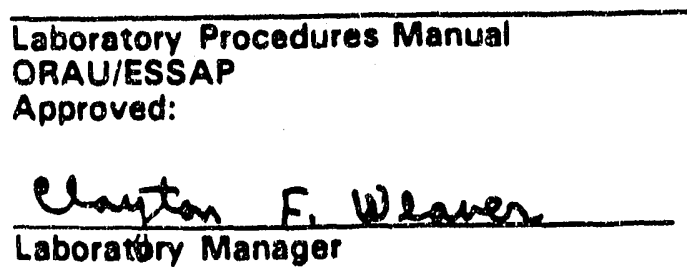

Laboratory Manager
Revision No. 6

Date: April 2, 1991

Page 2 of Section 15 


\subsubsection{Carbon Suspension}

Fume one $47 \mathrm{~mm} \mathrm{GA-6}$ or GA-4 metricel filter in $5 \mathrm{ml}$ of conc. $\mathrm{H}_{2} \mathrm{SO}_{4}$. Cool and dilute to $50 \mathrm{ml}$ with water.

2.2.5 Chromous Chloride, 3M:

See lab supervisor for procedure and instruction.

2.2.6 DBHQ, purification:

See lab supervisor for procedure and instruction.

2.2.7 DBHQ, 0.2M:

Dissolve $11.1 \mathrm{~g}$ purified DBHO in $250 \mathrm{ml}$ of 2-ethyl-1-hexanol.

2.2.8 Ethanol, 95\%:

May be avaifiable as such, or dilute $475 \mathrm{ml}$ of ethyl alcohol $10500 \mathrm{ml}$ and place in a squirt bottle.

2.2.9 Fusion Solution, $200 \mathrm{mg} \mathrm{NaHSO}$, and $0.5 \mathrm{ml} \mathrm{H}_{2} \mathrm{SO}_{4}$ per $2 \mathrm{ml}$ :

Dissolve $50 \mathrm{~g}$ of sodium acid sulfate (NaHSOd in about $300 \mathrm{ml}$ of reagent water. Slowly add $125 \mathrm{ml} \mathrm{H} \mathrm{HO}_{4}$, cool in a water bath if necessary. Dilute to $500 \mathrm{ml}$. Typically $2 \mathrm{ml}$ of solution supplies the required reagents.

2.2.10 HDEHP, $15 \%$ :

Measure $75 \mathrm{ml}$ of Bis(2-ethylhexyl) hydrogen phosphate (HDEHP) into a $500 \mathrm{ml}$ graduated cylinder. Dilute to $500 \mathrm{ml}$ with $\mathrm{n}$-heptane. Stir to mix.

2.2.11 Hydrazine-Sulfamic Acid Solution:

Caution: Handle hydrazine with extreme care.

Dissolve $97.1 \mathrm{~g}$ Sulfamic acid $\left(\mathrm{N}_{3} \mathrm{NSO}, \mathrm{H}\right)$ in about $400 \mathrm{ml}$ of reagent water. Dilute to $500 \mathrm{ml}$. Add $100 \mathrm{ml} 95 \%$ hydrazine. Wear gloves and work only in a hood when handling hydrazine.

Laboratory Procedures Manual

Revision No. 6

ORAU/ESSAP

Date: April 2, 1991

Approved:

Page 3 of Section 15
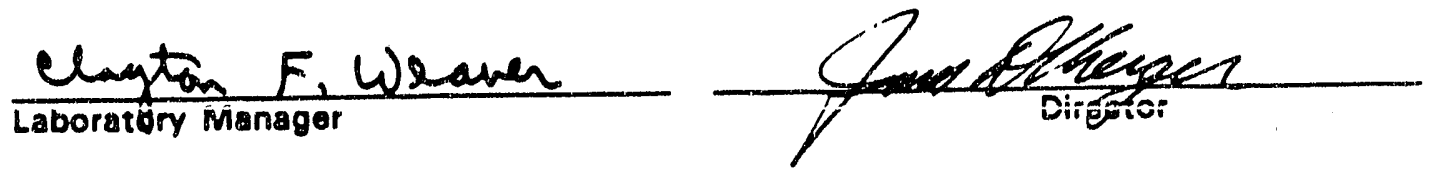
C-19

2.2.12 Hydrochloric acid, concentrated, 10M

2.2.13 Hydrochloric acid, 10M:

To $167 \mathrm{ml}$ reagent water, add $833 \mathrm{ml}$ conc. $\mathrm{HCl}$.

2.2.14 Hydrochloric acid, 3M:

To $750 \mathrm{ml}$ reagent water, add $250 \mathrm{ml}$ conc. $\mathrm{HCl}$.

2.2.15 Hydrofluoric acid, concentrated, $48 \%$ :

CAUTION: Skin contact with HF causes very severe burns.

2.2.16 Nitric Acid, concentrated, 16M.

2.2.17 Nitric Acid, 8M:

To $500 \mathrm{ml}$ reagent water, add $500 \mathrm{ml}$ conc. HNO 3 .

2.2.18 Nitric Acid, 4M:

To $750 \mathrm{ml}$ reagent water, add $250 \mathrm{ml}$ conc. $\mathrm{HNO}_{3}$.

2.2.19 Perchloric Acid, concentrated, $72 \%$.

CAUTION: Conc. HC10, seacts violently with an organic material li.e. paper, skin, alcoholl. Hot $\mathrm{HC10}$, should be used only in rated hoods with washdown after use.

2.2.20 Reprecipitating Solution

Dissolve $135 \mathrm{~g}$ of anhydrous potassium sulfate $\left(\mathrm{K}_{2} \mathrm{SO}_{4}\right)$ in $915 \mathrm{ml}$ of reagent water and $50 \mathrm{ml}$ of concentrated hydrochloric acid (HCl) when cool add reagent water to give a total volume of $1000 \mathrm{ml}$.

Laboratory Procedures Manual

ORAUIESSAP

Approved:

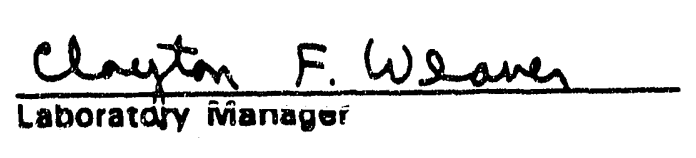

Revision No. 6

Date: April 2, 1991

Page 4 of Section 15 


\subsubsection{Substrate Suspension}

Dilute $10 \mathrm{mg}$ of $\mathrm{Ce}$ (purified if necessary) and $20 \mathrm{ml}$ of $12 \mathrm{M} \mathrm{HCl}$ to $250 \mathrm{ml}$ with reagent water. Dilute $8 \mathrm{ml}$ of $48 \% \mathrm{HF}$ to $250 \mathrm{ml}$ of reagent water. Combine the solutions and add $2 \cdot 3 \mathrm{ml}$ of carbon suspension. Vigorously shake the substrate solution prior to use. The carbon in the substrate permits visual observation of the area of the otherwise white CeF, on the white background of the filter.

2.2.22 Uranium Stripping Solution: Perchloric-Oxalic Solution

Dissolve $20 \mathrm{~g}$ oxalic acid in $300 \mathrm{ml}$ of reagent water, add $50 \mathrm{ml}$ of concentrated perchloric acid, stir well, and dilute to $500 \mathrm{ml}$ with reagent water.

2.3 If the analysis is for uranium and/or thorium go to references 3 and 4 and prepare the tracers.

$$
\text { Thorium-234 Tracer }
$$

2.3.1 Dissolve one pound of reagent-grade uranyl nitrate hexahydrate in 100 $\mathrm{ml}$ of concentrated hydrochloric acid and evaporate to dryness.

2.3.2 Dissolve the uranyl chloride in $500 \mathrm{ml}$ of $9.6 \mathrm{M}$ hydrochloric acid and add the solution to the column containing four pounds of Dowex $1 \cdot \times 4$.

2.3.3 Elute with $9.6 \mathrm{M}$ hydrochloric acid at a flow rate of $10.15 \mathrm{ml}$ per minute until a total of $2400 \mathrm{ml}$ is collected. Discard. Wait approximately 30 days.

2.3.4 Elute with 2 liters of $9.6 \mathrm{M}$ hydrochloric acid. Evaporate the eluate to $2.3 \mathrm{ml}$ transferring to a $250 \mathrm{ml}$ beaker during the process.

2.3.5 Rinse the original beaker with $25 \mathrm{ml}$ of concentrated nieric acid and add the rinse to the $250 \mathrm{ml}$ beaker.

2.3.6 Evaporgte to about $5 \mathrm{ml}$.

2.3.7 Add $25 \mathrm{ml}$ of $8 \mathrm{M}$ nitric acid and heat to boiling. Cool.
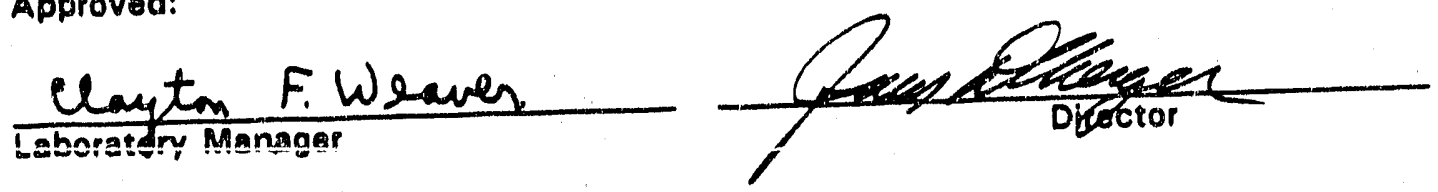
2.3.8 Transfer to a $250 \mathrm{ml}$ separatory funnel. Finse beaker with $15 \mathrm{ml}$ of $8 \mathrm{M}$ nitric acid and add to separatory funnel.

2.3.9 Extract the thorium with $50 \mathrm{ml}$ of $30 \%$ Aliquot-336 (NO, $)$ in xylene and discard the aqueous phase. Shake 5 minutes all or approximately 5 minutes for separation.

2.3.10 Wash the organic phase with $25 \mathrm{ml}$ of $8 \mathrm{M}$ nitric acid and discard the aqueous phase. Shake 3 minutes and allow 3 minutes for seperation.

2.3.11 Strip the thorium with $50 \mathrm{ml}$ of $10 \mathrm{M} \mathrm{HCl}$. Repeat for a total of 3 strips. [Shake 5 minutes and allow 3 minutes for seperation each time] Discard the organic phose.

2.3.12 Combine the three strips wash with $25 \mathrm{ml}$ of $30 \%$ Allquot -336 solution. Discard the organic phase. Wash 2 minutes and let stand 2 minutes.

2.3.13 Place the aqueous phase in a $250 \mathrm{ml}$ Vycor flask, add $5 \mathrm{ml}$ of $72 \%$ perchloric acid, and two SiC boiling chips.

2.3.14 Evaporate the solution to fumes. Add concentrated nitric acid dropwise during the fuming stage.

2.3.15 Add $5 \mathrm{ml}$ each of concentrated hydrochloric and nitric acid to the flask and re-evaporate to about $2 \mathrm{ml}$.

2.3.16 Add $15 \mathrm{ml}$ concentrated nitric acid and boil down to about $5 \mathrm{ml}$. Cool.

2.3.17 Add $25 \mathrm{ml}$ of water. Filter with a DM 450 membrane filter. Wash flask with $25 \mathrm{ml}$ of water and filter. This provides about $50 \mathrm{ml}$ of Th-234 tracer containing approximately $10^{4} \mathrm{pCi} / \mathrm{ml}$.

\section{IJ-232 Tracer}

2.3.18 Add $10 \mathrm{ml}$ of concentrated sulfuric acid to $5 \times 10^{3} \mathrm{dpm}$ of U.232 in a $250 \mathrm{ml}$ flask and evaporate to fumes.

2.3.19 Add $25 \mathrm{~g}$ of anhydrous potassium sulfate and 2 drops of $72 \%$ perchloric acid.

2.3.20 Heat gently over a blast lamp while swirling until perchloric acid has been decomposed. Cool melt.

2.3.21 Add $150 \mathrm{ml}$ of water and heat to boiling. Cool.

2.3.22 Add $1 \mathrm{ml}$ of $30 \%$ hydrogen peroxide and transfer to a $250 \mathrm{ml}$ volumetric flask.

\section{Laboratory Procedures Manugl ORAU/ESSAP \\ Approved:}

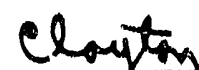

F. Wraven Laboratory Manager
Rovision No. 6

Date: April 2, 1991

Page 6 of Section 15 
2.3.23 Add $20 \mathrm{ml}$ of $.45 \%$ barlum chloride dehydrate while swirling and dilute to $2.50 \mathrm{ml}$.

\subsection{Sample Decomposition}

2.4.1 Weigh $1.0^{\prime} \mathrm{g}$ of the .35 mesh soil into a dry semiflat bottomed platinum dish.

2.4.2 Add $0.5 \mathrm{ml}$ of concentrated HNO, a few drops at a time as fast as the reaction will permit.

2.4.3 Heat gently until the reaction has subsided and the soil remains just moist.

2.4.4 Add an appropriate amount of the tracers. Dry completely between additions of different tracers. Enter theoretical vield(s) on data sheet(s).

2.4.5 Add $5.0 \mathrm{~g}$ anhydrous $\mathrm{KF}$ to the soll. Slowly add $4.0 \mathrm{ml} 48 \% \mathrm{HF}$ to the soil and $\mathrm{KF}$.

2.4.6 Hold the dish over a low temperature blast burner. Heat gently and agitate to mix into a thickening slurry. heat as quickly as possible to dryness.

2.4.7 Place the platinum dish on a ring stand using a nichrome triangle.

2.4.8 Use a high temperature blast burner in order to apply as much heat as possible, with limited splattering, to bring the temperature to about $900^{\circ} \mathrm{C}$. This should take about 4 minutes. The total dissolution process will take another 2 minutes. Swirl the hot melt to ensure removal of sample clinging to the sides of the dish.

2.4.9 Remove the molf from the burner and swirl gently around the dish to form a thin layer upon cooling. (Never sit hot platinum on iron).

2.4.10 After the cake has cooled to room cemperature, add a total of $7.0 \mathrm{ml}$ of concentrated $\mathrm{H}_{3} \mathrm{SO}$, to the fluoride cake. The acid should be added to the edge of the cake and allowed to run to the bottom of the dish. Because of the evolution of Sif." this step must be done in a hood.

2.4.11 After the addition of the $\mathrm{H}_{2} \mathrm{SO}_{4}$, heat as fast as frothing will allow until the fluoride cake is totally dissolved and a thick slurry forms.

'For environmental samples, a $10 \mathrm{~g}$ aliquot may be used. The quantity of reagents used must be increased proportionally. This comment applies only through the pyrosulfate fusion.

Laboratory Procedures Manual

Revision No. 6

ORAU/ESSAP

Date: April 2. 1991

Approved:

Page 1 of Section 15
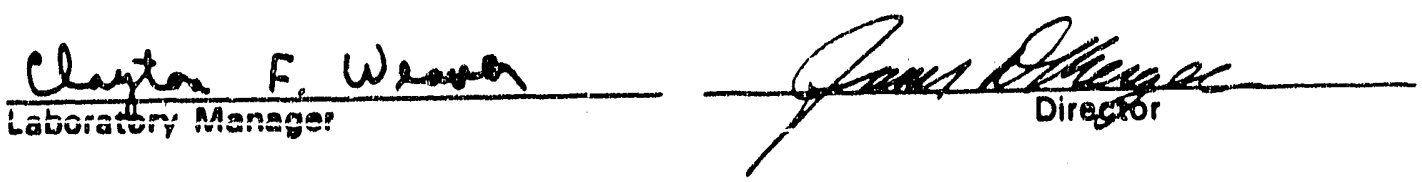
2.4.12 Remove from the heat and add $3.0 \mathrm{~g}$ of anhydrous $\mathrm{Na}_{2} \mathrm{SO}_{4}$ to the slurry. Using a hot plate geritly, heat the sample until the $\mathrm{Na}_{2} \mathrm{SO}_{4}$ dissolvos. Place the sample over the blast burner with small flame and heat until the slurry begins to turn a golden brown. Slowly increase the temperature until the slurry is completely melted, then maintain this temperature for approximately 1 minute.

2.4 .13 To a 1 liter beaker, add $500 \mathrm{ml}$ of reagent water, $150 \mathrm{ml}$ of $12 \mathrm{M} \mathrm{HC1}$. and a teflon stirring bar. Cover with a watch glass, place on a hot plate. and bring the solution to a boll.

2.4.14 Carefully add the pieces of pyrosulfate cake to the boiling solution, Allow cake to dissolve then proceed.

2.4.15 With rapid stirring, slowly add $3.0 \mathrm{~g}$ of $\mathrm{K}_{2} \mathrm{~S}_{2} \mathrm{O}_{3}$ and boil the solution to $550 \mathrm{ml}$.

2.4.16 Add $20.0 \mathrm{~g}$ of anhydrous $\mathrm{Na}_{2} \mathrm{SO}_{4}$ and wait until the $\mathrm{Na}_{2} \mathrm{SO}$. dissolves.

2.4.17 Add $50 \mathrm{ml}$ of $0.45 \% \mathrm{BaCl}_{2} 2 \mathrm{H}_{2} \mathrm{O}$ in five $10 \mathrm{ml}$ portions. Boil for approximately 5 minutes after each addition.

2.4.18 Using a teflon filtering chimney, filter the hot solution through a sup or $45047 \mathrm{~mm}$ membrane filter of 0.45 micron porosity.

2.4.19 Wash the precipitate left in the beaker onto the Supor 450 filter using $0.5 \% \mathrm{H}_{2} \mathrm{SO}_{4}$.

2.4.20 For thorium analysis go to BaSO, reprecipitation section, Stop 2.5.1.

2.4.22 For Pu go to Step 2.7.1. Am go to Step 2.8.1.

2.4.23 For uranium proceed immediately to Step 2,6.1.

\subsection{Barium Sulfate Reprecipitation for Thorium}

2.5.1 Place the filter paper with the $\mathrm{BaSO}_{4}$ in a $250 \mathrm{ml}$ Erlenmeyer flask.

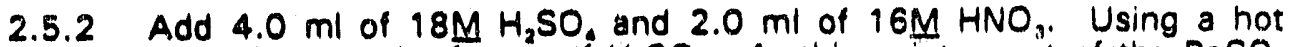
plate, heat to the furnes of $\mathrm{H}_{2} \mathrm{SO}_{4}$. At this point, most of the BaSO. should be in solution. If there is any sign of organics, add $\mathrm{HNO}_{3}$ dropwise until the organics are oxidized.

2.5.3 Add $3.0 \mathrm{ml}$ of 1:1 $\mathrm{HNO},-\mathrm{HCl}$ solution to dissolve any elemental platinum that may have been filtered with the BaSO.
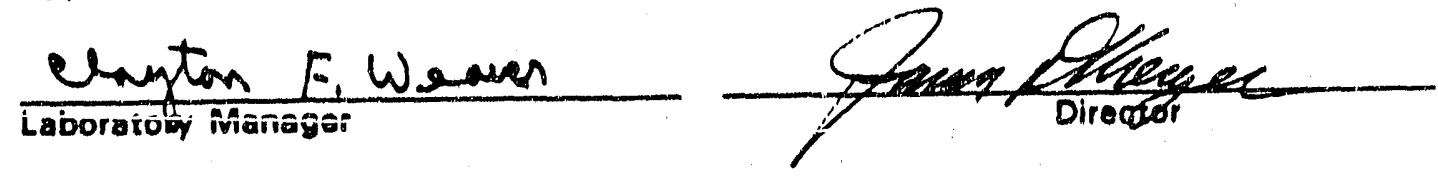
2.5.4 After the platinum dissaives, use a small blast burner flame to dissolve any remaining BaSO.

2.5.5 Cool to room temperature.

2.5.6 Add $60 \mathrm{ml}$ of the reprecipitating solution and $1.0 \mathrm{ml}$ of $25 \% \mathrm{~K}_{2} \mathrm{~S}_{2} \mathrm{O}_{6}$ solution ${ }^{2}$ bringing the solution to a boil for 2 minutes.

2.5.7 Filter the hot solution through a Supor 450 filter in a teflon filtering chimney. Wash the precipitate from the flask onto the filter using $0.5 \%$ $\mathrm{H}_{2} \mathrm{SO}_{4}$.

2.5.8 Plack the filter with the BaSO, precipitate into a $250 \mathrm{ml}$ Erlenmeyer flask.

\section{LIQUID - LIQUID EXTRACTION OF THORIUM}

2.5.9 To the flask containing the BaSO, procipitate, add $2.0 \mathrm{ml}$ of $16 \mathrm{M}$ HNO, $4.0 \mathrm{ml}$ of $12 \mathrm{M} \mathrm{HClO}$, and heat gently on a hot plate to destroy the fiter paper and dissolve the BaSO. Sometimes the precipitate does not dissolve easily. It then becomes necessary to replace the $\mathrm{HClO}$, lost in heating. The final volume of $\mathrm{HClO}$, should be about $4.0 \mathrm{ml}$. It has been observed, however, that the procedure can work with olose to 8.0 $\mathrm{ml}$ of $\mathrm{HClO}$. Some silicas may not dissolve even in the excess $\mathrm{HClO}$.. but will dissolve when added to Al( $\left.\mathrm{NO}_{3}\right)_{3}$.

2.5.10 Mix $6.0 \mathrm{ml}$ of $16 \mathrm{M} \mathrm{HNO}$, with $50 \mathrm{ml}$ of $2.2 \mathrm{M} \mathrm{Al}\left(\mathrm{NO}_{3}\right)_{\text {, }}$ and warm to $50^{\circ}$ to $60^{\circ} \mathrm{C}$. Remove the sample flask from the heat and carefully add the Al(NO$)_{3}$, solution. If a precipitate does not form, cool the sample to room tempersture then proceed. If a precipitate does form, heat the solution to near boiling until the precipitate dissolves. Replace the volume loss with reagent water. Too much lost volume will cause the Al(NO,), to crystallize. Cool the solution to room temperature then proceed.

2.5.11 Put $25 \mathrm{ml}$ of $2.2 \mathrm{M}$ Al(NO, $)_{3}$ in a $250 \mathrm{ml}$ separatory funnel.

2.5.12 Add $6.0 \mathrm{ml}$ of $25 \% \mathrm{NaNO}_{2}$ (prepared fresh daily) to the Al(NO, $)_{3}$ in the funnel followed as quickly as possible by the cooled sample. This is necessary to prevent the excess loss of $\mathrm{HNO}_{2}$ which can affect the thorium recovery.

2.5.13 Add $50 \mathrm{ml}$ of $30 \%$ (y/v) Aliquot-336 in xylene, mix gently, and let stand for 10 minutes.

2.5.14 Using a mechanical shaker, extract the thorium from the aqueous phase by shaking for 3 minutes. Allow the phases to separate for 3 minutes.

Laboratory Proceduras Manual

ORAUIESSAP

Approved:

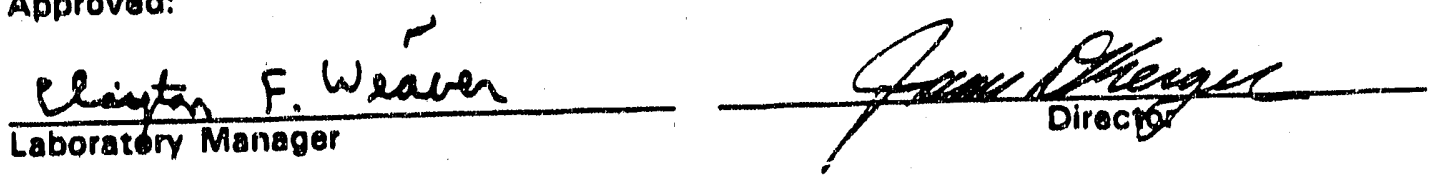

Revision No. 6 Dare: April 2, 1991 
Drain and discard the aqueous phase (bottom layer).

2.5.15 Wash the organic phase with three $10.0 \mathrm{ml}$ portions of $8 \mathrm{M}$ HNO, washings (bottom layer). Do not allow the organic phase to enter the" stopoock.

2.5.16 Extract the thorium from the organio laver with three $50 \mathrm{ml}$ washings of $10 \mathrm{M} \mathrm{HCl}$. Collect the $10 \mathrm{M} \mathrm{HCl}$ in a $250 \mathrm{ml}$ Erlenmever flask. Leave about $0.5 \mathrm{ml}$ of the $\mathrm{HCl}$ in the separatory funnel.

2.5.17 Place the flask containing the $\mathrm{HCl}$ washing on a hot plate. Add $2 \mathrm{ml}$ of fusion solution and a stir rod.

2.5.18 Heat the sample until $\mathrm{Nu}, \mathrm{S}, \mathrm{O}$, crystals form and only a fow drops of $\mathrm{H}_{2} \mathrm{SO}_{4}$ are on the rim of the flask. Cool to room temperature and deposit using an approved method. Cerium fluoride or neodymium fluoride can be used for deposition.

CERIUM FLUORIDE DEPOSITION FOR TH, PU, AM, AND NP

2.5.19 Dissolve the pyrosulfate crystals in $10 \mathrm{ml}$ of $3 \mathrm{M} \mathrm{HCl}$ using $50^{\circ}$ to $60^{\circ} \mathrm{C}$ heat for 3 to 5 minutes. Do not allow the volume to drop below 8 or $9 \mathrm{ml}$.

2.5.20 Transfer the solution to a round-bottom polycarbonate centrifuge tube with reagent water. Total volume should be about $12 \mathrm{ml}$.

2.5.21 Add $\mathrm{C}_{\mathrm{e}}$ carrier. (This should be equivalent to $50 \mathrm{\mu g}$ of $\mathrm{Ce}$ ).

2.5.22 Add $5 \mathrm{ml}$ of $48 \% \mathrm{HF}$, mix well, and let stand for a minimum of 10 minutes. Do not wait over 30 minutes. Before proceeding, check the color to be sure red or pink is not present.

2.5.23 Mount the CeF, precipitate on a HT-200 filter in the following manner:

1) Insert the HT-200 filter bottom side up into a twist lock funnel. The most porous side of the filter is faceup in the filter box. Simply turn the filter over before placing in the filtering funnel.

2) Add 2 to $4 \mathrm{ml}$ of $95 \%$ ethanol to the filter and apply suction.

\footnotetext{
${ }^{2} \mathrm{The} \mathrm{K}_{2} \mathrm{~S}_{2} \mathrm{O}_{1}$ solution must be prepared fresh for each batch analysis.
}

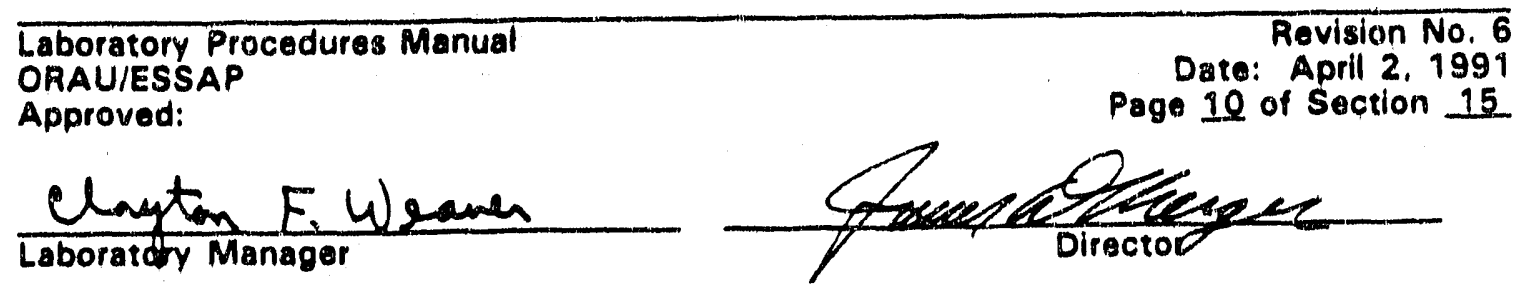




\section{C-26}

3) To the center of the fliter, add a total of $10 \mathrm{ml}$ of substrate? suspension under reduced suction. This should be dorie $3.3 \mathrm{ml}$ at a time. Apply hard suction" at the beginning of the next two additions. Then reduce suction as soon as the substrate has been dispensed. After the substrate has filtered, apply hard suction for about 30 seconds in order to firmly seat the substrate on the fllter.

4) Apply hard suction, then add a well-shaken sample to the filter. Atter the sample has been added, reduce the suction to allow the sample to filter slowly. While the sample is filtering, wash the centrifuge tube with 2 to $4 \mathrm{ml}$ of rosgent water and add to the filtering sample.

5) After the sample has filtered, add $5 \mathrm{ml}$ of reagent water, under hard suction, indirectly to the filter. The water helps remove residual HF from the fllter.

6) After the water has passed, add $2.104 \mathrm{ml} 95 \%$ ethanol under hard suction.

7) Remove the filter and place on a stainless steol disc using double stick tape. If tape is not used, the HT.200 filter will curl affecting the resolution and counting efficiency for a given sample.

\subsection{Uranium}

2.6.9 If the flltrate that has been sitting overnight filter through a Supor 450 membrane filter in a teflon filtering chimney. Discard precipitate.

2.6.2 Add $5.0 \mathrm{~g}$ of $\mathrm{K}_{2} \mathrm{~S}_{2} \mathrm{O}_{6}$.

2.6.3 Cover the beaker with a watch glass and boll for 20 minutes to volatilize the $\mathrm{SO}_{2}$.

2.6.4 When the odor of $\mathrm{SO}$, has largely disappeared (20 min.), add 4 drops of $1 \%$ aqueous safranin-0. This should produce a reddish color.

${ }^{3}$ The substrate should be hand shaken for about 2 minutes before being used with the sample.

Hard suction is necessary to keep the substrate in place. If the substrate is displaced, the resolution for a sample can be reduced. A degraded spectrum reduces the reliability of the analytical data.

Laboratory Procedures Manual

Revision No. 6

ORAU/ESSAP

Date: April 2, 1991

Approved:

Page 11 of Section 15
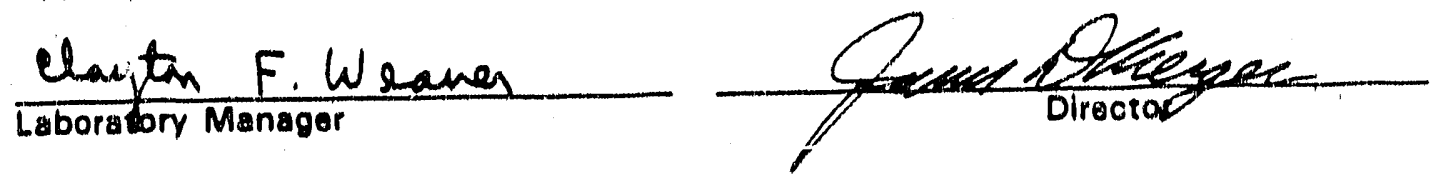
2.6.5 Add 20\% $\mathrm{TICl}$, dropwise until the reddish color ohanges to the colorless leuco form. The uranium is reduced from the +6 state to the +4 state for later coprecipitation with BaSO.

2.6.6 Add 15 drops of $3 \mathrm{M} \mathrm{CrCl}_{2}$ from a pipette under the watch glass. This reduces titanium which acts as a holding reductant for +4 uranium during the BaSO, precipitation.

2.6.7 Add 2 drops of $1 \%$ aqueous safranin.0. The solution should have the definite green of +3 chromium. If the solution is red or pink, add $20 \%$ TiCl, dropwise uritil the solution turns green.

2.6.8 Cover with a watch glass and heat the solution to boiling.

2.6.9 Add $20 \mathrm{~g}$ of anhydrous $\mathrm{Na}_{2} \mathrm{SO}_{4}$ ar.d wait for the $\mathrm{Na}_{2} \mathrm{SO}_{4}$, to dissolve.

2.6.10 While seirring, add $50 \mathrm{ml}$ of barium as $0.45 \% \mathrm{BaCl}, 2 \mathrm{H}_{2} \mathrm{O}$ in five $10 \mathrm{ml}$ portions. Boil the solution for approximarely 5 minutes atter each addition.

2.6.11 Using a teflon filtering chimney, filter the hot solution through $47 \mathrm{~mm}$ Supor 450 membrane filter and rinse with $0.5 \% \mathrm{H}_{2} \mathrm{SO}_{4}$.

2.6.12 Place the BaSO, precipitate in a $250 \mathrm{ml}$ flask and discard the filtrate.

LIQUID - LIQUID EXTRACTION OF URANIUM

2.6.13 To the fiask containing the BaSO, precipitate, add $2.0 \mathrm{ml}$ of $16 \mathrm{M} \mathrm{HNO} \mathrm{H}_{3}$ $4.0 \mathrm{ml}$ of $12 \mathrm{M} \mathrm{HClO}$, and heat gently on a hot plate to destroy the filter paper and dissolve the BaSO. Sometimes the precipitate does not dissolve easily. It then becomes necessary to replace the $\mathrm{HClO}$, lost in hearing. The final volume of $\mathrm{HClO}$, should be about $4.0 \mathrm{ml}$. it has been observed, howover, that the procedure can work with close to 8.0 $\mathrm{ml}$ of $\mathrm{HClO}$. Some sillicas may not dissolve even in the excess $\mathrm{HClO}$, but will dissolve when added to Al(NO, $)_{3}$.

2.6.14 Mix $6.0 \mathrm{ml}$ of $16 \mathrm{M} \mathrm{HNO}$, with $50 \mathrm{ml} \mathrm{of} 2.2 \mathrm{M} \mathrm{Al}\left(\mathrm{NO}_{3}\right)_{3}$ and warm to $50^{\circ}$ to $60^{\circ} \mathrm{C}$. Remove the sample flask from the heat and carefully add the Al(NO, ), solution. If a precipitate does not form, cool the sample to room temperature then proceed. If a precipitate does form, heat the solution to near boilling until the precipitate dissolves. Replace the volume loss with reagent water. Too much lost volume will cause the Al(NO,), to orystallize. Cool the solution to room temperature then procoed.

2.6.15 Put $25 \mathrm{ml}$ of $2.2 \mathrm{M} \mathrm{Al}\left(\mathrm{NO}_{3}\right)$, in $250 \mathrm{ml}$ separato.y funnel.

Laboratory Procadures Manual

Revision No. 6

ORAU/ESSAP

Approved:

Date: April 2, 1991
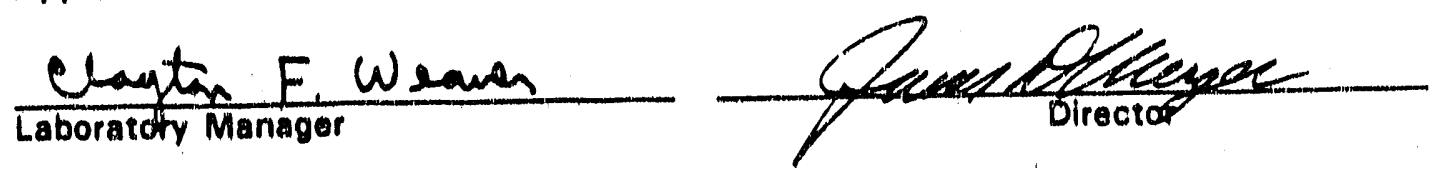


\section{C-28}

2.6.16 Add $6.0 \mathrm{ml}$ of $25 \%$ (w/W) NaNO, (prepared fresth daily) to the Al(NO,), in the funnel, followed as quickly as possible by the cooled sample. This is necessary to prevent the excess loss of $\mathrm{HNO}_{2}$ which can affect the uranlum recovery.

2.6.17 Add $50 \mathrm{ml}$ of Aliquot. 336 in $30 \%$ xylene, mix gently, and let stand for 10 minutes.

2.6.18 Using a mechanical shaker, extract the uranlum from the aqueous phase by shaking for 3 minutes. Allow the phases to separate for 3 minutes. Drain and discard the aqueous phase (bottom layer).

2.6.19 Scrub the organic layer 1 time with $50 \mathrm{ml}$ of $10 \mathrm{M} \mathrm{HCl}$ for 3 minutes. Drain and discard the aqueous phase (bottom layer). When draining the aqueous phases, do not allow the organic liquid to enter the stopcock outlet. Leave about 0.25 to $0.5 \mathrm{ml}$ of the aqueous phase in the separatory funnel."

2.6.20 Strip the uranium from the organic layer by adding $50 \mathrm{ml}$ of perchloric-oxalic acid solution and shaking for 3 minutes. Let the phases separate for 3 minutes then drain the aqueous phase (bottom layer). leaving about 0.25 to $0.5 \mathrm{ml}$ of it in the funnel, and proceed with the uraniurn analysis.

2.6.21 Wash the organic phase for 3 minutes with $25 \mathrm{ml}$ reagent water. Allow the phases to separate, drain the aqueous phase (bottom layer) and combine the two aqueous strips.

2.6.22 To the aquenus strips, add $2 \mathrm{ml}$ of fusion solution and a stir rod.

2.6.23 Heat on a hot plate until $\mathrm{Na}_{2} \mathrm{~S}_{2} \mathrm{O}$, crystals form and only a few drops of $\mathrm{H}_{2} \mathrm{SO}$, are on the rim of the flask. Cool to room temperature and duposit using Cerlum fluoride deposition.

CERIUM FLUOAIDE DEPOSITION FOR URANIUM ONLY

2.6.24 Dissolve the pyrosulfate orystals in $10 \mathrm{ml}$ of $3 \mathrm{M} \mathrm{HCl}$ using $50^{\circ}$ to $60^{\circ} \mathrm{C}$ heat for 3 to 5 minutes. Do not allow the volume to drop below 8 to $9 \mathrm{ml}$.

Leaving some of the aquedus phase in the separatory funnel will prevent organic carryover into the sample. While the organics can be easily oxidized with concentrated HNO, the procedure is quicker and the analyst is less likely to break the flask by the addition of HNO, to a hot sample.

Laboratory Procedures Manual

Revision No. 6

ORAU/ESSAP

Date: April 2, 1991

Approved:

Page 13 of Section 15
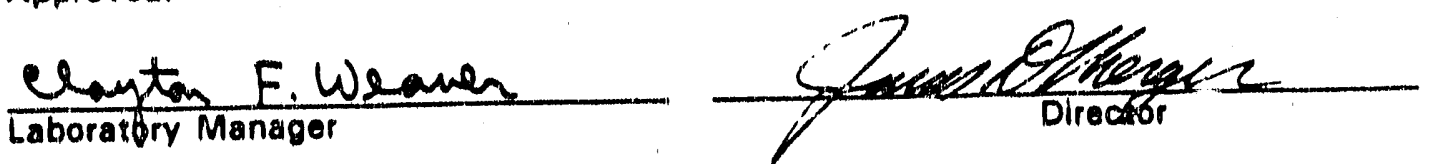
2.6.25 Transfer the solution to a round-bottom polycarbonate centrifuge tube with reagent water. Total volume should be about $12 \mathrm{ml}$.

2.6.26 Add 1 drop of $0.1 \%$ aqueous Satranin 0 and 1 drop of $20 \% \mathrm{TiCl}$, A slight purple color from the TiCl, should be noticeabie. If a red or pink color persists, add another drop of $\mathrm{TiCl}_{3}$.

2.6.27 Add Ce carrier. (This should be equivalent to $50 \mu \mathrm{g}$ of $\mathrm{Ce} .1$

2.6.28 Add $5 \mathrm{ml}$ of $48 \% \mathrm{HF}$, mix well, and let stand for minimum of 10 minutes. Do not wait over 30 minutes. Before proceeding, check the color to be sure red or pink is not present.

2.6.29 Mount the Cef, precipltate on a HT-200 filter in the following marner:

1) Insert the HT-200 filter bottom side up into a twist lock funnel. The most porous side of the filter is faceup in the filter box. Simply turn the filter over before placing in the filtering funnel.

2) Add 2 to $4 \mathrm{ml}$ of $95 \%$ ethanol to the filter and apply suction.

3) To the center of the filter, add a total of $10 \mathrm{ml}$ of substrate ${ }^{\circ}$ suspension under reduced suction. This should be done $3.3 \mathrm{ml}$ at a time. Apply hard suction' at the beginning of the next two additions. Then reduce suction as soon as the substrate has beerl dispensed. After the substrate has filtered, apply hard suction for about 30 seconds in order to firmly seat the substrate on the fllter.

4) Apply hard suction, then add a well-shaken sample to the filter. After the sample has been added, reduce the suction to allow the sample to filter slowly. While the sample is filtering, wash the centrifuge tube with 2 to $4 \mathrm{ml}$ of reagent water and add to the filtering sample.

5) After the sample has filtered, add $5 \mathrm{ml}$ of reagent water, under hard suction, indirectly to the filter. Thie water helps remove residual HF from the filter.

6) After the water has passed, add 2 to $4 \mathrm{ml}$ of $95 \%$ ethanol under hard suction.

7) Remove the filter and place on stainless steol disc using double stick tape. If tape is not used, the HT-200 filter will curl affecting the resolution and counting efficiency for a given sample,
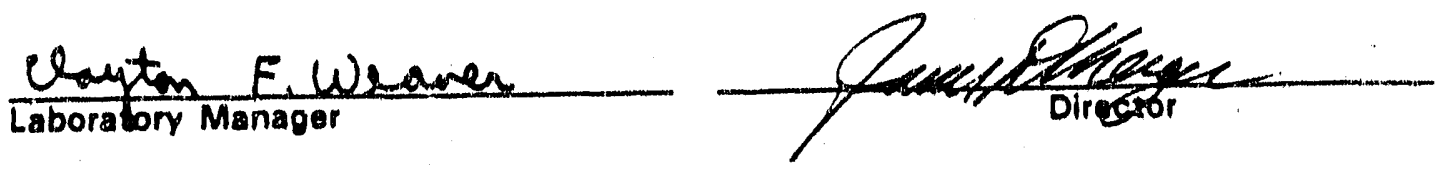
2.7 Sequential extraction of Americium, Plutonium, Neptonium and/or Thorium. Proceed until the desired element is eluted, then proceed as directed.

2.7.1 Prepare the sample the same way as for uranium procedure through step 8 of the pyrosulfate cake dissolution and barium sulfate precipitation.

2.7.2 Place the filter into a $250 \mathrm{ml}$ Erlenmeyer flask.

2.7.3 Add $30 \mathrm{~m} !$ of $72 \% \mathrm{HClO}$, and start with medium heat (settings of 3 or 4). As the filter disintegratiss and forms a brown scum, slowly increase heat until the acid begins to boil (a setting of about 6). The brown material should disappear and the solution should be clear and colorless or light green/yellow. Remove from hear and allow to coo while preparing for the next step.

2.7.4 Transfer the solution into a $60 \mathrm{ml}$ separatory funnel containing $10 \mathrm{ml}$ of $15 \%$ HDEHP in N-heptane and extract for 5 minutes. Let it sit 3 minutes.

2.7.5 Add $5 \mathrm{ml}$ of $72 \% \mathrm{HClO}_{4}$. Shake 3 minutes and allow 3 minutes for seperation. Discard the bottom portion. Repeat this step once.

2.7.6 Add $10 \mathrm{ml}$ of $4 \mathrm{M}$ nitric acid containing $1.0 \mathrm{ml} 25 \%$ sodium nitric (prepared fresh daily). Shake and let stand for 2 minutes each. Save the bottom layer for Americium. Add $10 \mathrm{ml}$ of $4 \mathrm{M}$ nitric acid. Shake and let stand 2 minutes each. Combine this bottom laver with the preceeding wash for Americium.

2.7.7 Americium only: Transfer the two strips from 2.7 .6 into a $250 \mathrm{ml}$ erlenmeyer flask containing $1 \mathrm{ml}$ at $72 \%$ perchloric acid and $2 \mathrm{ml}$ of fusion solution. Evaporate to dryness on a hot plate. Go to the cerium fluoride deposition, step 2.5.19.

2.7.8 Americium has now been seperated from the organic phase. Add 10 $\mathrm{ml}$ of $4 \mathrm{M}$ nitric acid and $2 \mathrm{ml}$ of hydrazine sulfamic acid solution. Shake and let stand 2 minutes each. Discard the bottom layer.

The substrate should be hand shaken for about 2 minutes before being used with the sample.

'Hard suction is necessary to keep the substrate in place. If the substrate is displaced, the resolution for a sample can be reduced. A degraded spectrum reduces the reliability of the analytical data.

Laboratory Procedures Manual

ORAU/ESSAP

Approved:

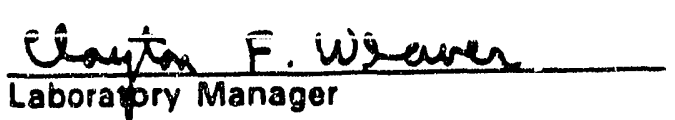

Revision No, 6

Date: April 2, 1991

Page 15 of Section 15 
2.7.9 In order, add, $5 \mathrm{ml}$ of $0.2 \mathrm{M} \mathrm{DBHO}, 10 \mathrm{ml}$ of $4 \mathrm{M}$ nitric acid and $2 \mathrm{ml}$ of hydrazine-sulfamic acid solution. Shake 5 minutes and let seperation go for 3 minutes. Save the bottom layer for Plutonimum analysis. Add $10 \mathrm{ml}$ of $4 \mathrm{M}$ nitric acid and $2 \mathrm{ml}$ of hydrazine-sulfamic acid solution. Shake as above. Combine the bottom layer with the prior plutonium extract above.

2.7.10 For Plutonium only: Combine the two extractions from step 2.7.9 in a $250 \mathrm{ml}$ erlenmeyer flask containing $2 \mathrm{ml}$ of fusion solution, $2.5 \mathrm{ml}$ nitric acid and $2.5 \mathrm{ml}$ hydrochloric acid. heat until a yellow color appears. Add another $2.5 \mathrm{ml}$ each of nitric and hydrochloric acids and $1 \mathrm{ml}$ of $72 \%$ perchloric acid. Evaporate to dryness. Go to step 2.5.19.

2.7.11 Plutonium has now been seperated from the organic phase.

Add $15 \mathrm{ml}$ toluene to the HDEHP/DBHO mixture. (A precipitate will form but it should redisolve subsequently).

Shake with $15 \mathrm{ml}$ of $4 \%$ oxalic acid for 3 minutes. Allow 4 minutes for separation then drain the lower layer into a $250 \mathrm{ml}$ erlenmeyer flask.

Add $2 \mathrm{ml}$ of fusion solution to the combined strips in the erlenmeyer flask. Add $2 \mathrm{ml}$ of $\mathrm{HClO}_{4}$ and heat to perchloric acid fumes.

Remove from heat and cool sufficientiy to allow addition of $1 \mathrm{ml}$ of $\mathrm{HNO}_{3}$. Return to heat to destroy organic material. (MOre $\mathrm{HNO}_{3}$, and heat may be required).

\subsection{Calculations}

2.9.1 The samples analyzed by this techniowe are spiked with radionuclides which are standardized in this laboratbly using NIST traceable materials. The calculations are then direct proputtions. The data sheets are given on pages 15.17 through 15-20 ASIC programs named AMER, PLUTON, URAN and THOR are used to calculate the activities, errors, and minimum detectable activities for these nuclides.

Cinton Filizaver

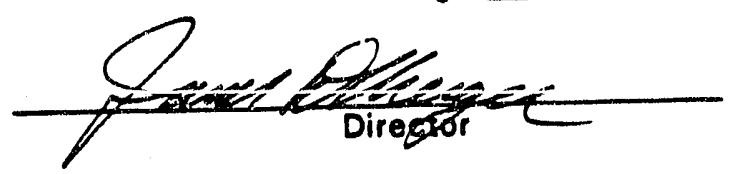


AMERICIUM ISOTOPIC CALCULATION SHEET

\begin{tabular}{|c|c|c|c|c|c|c|c|c|}
\hline \multirow[t]{2}{*}{ SAMPLE I. } & & & & & \multirow[b]{2}{*}{241} & \multirow[b]{2}{*}{243} & \multirow[b]{2}{*}{241} & \multirow[b]{2}{*}{243} \\
\hline & 241 & 243 & 241 & 243 & & & & \\
\hline \multicolumn{9}{|l|}{ GCNTS } \\
\hline \multicolumn{9}{|l|}{$B K G$} \\
\hline \multicolumn{9}{|l|}{ NCNTS } \\
\hline \multicolumn{9}{|c|}{$\begin{array}{l}D C i \text { OF } \\
243 \text { ADOED }\end{array}$} \\
\hline \multicolumn{9}{|c|}{$\begin{array}{l}\text { SAMPLE-QUANTITY: } \\
(I L \text { OR ( }) C \text { ORY }\end{array}$} \\
\hline \multicolumn{9}{|c|}{$\begin{array}{l}\text { SAMPLE-QUANTITY: } \\
\text { ASHED ( }) G\end{array}$} \\
\hline \multicolumn{9}{|l|}{ TIME } \\
\hline \multicolumn{9}{|l|}{$\begin{array}{l}\text { ACTIVITY IN } \\
\text { pCi/unit }\end{array}$} \\
\hline \multicolumn{9}{|l|}{$\begin{array}{l}\text { ERROR IN } \\
\mathrm{PCi} / \text { unit }\end{array}$} \\
\hline $\begin{array}{l}M D A \quad \mathbb{N} \\
D C i / \text { Init }\end{array}$ & & & & & , & & & \\
\hline
\end{tabular}

Activity of individual isotopes is calculated using o direct ratio of the counts and octivity of Am 243 odded to eoch somple. and the counts of eoch isotopic region of interest. FOR EXAMPLE, Am 241 would be colculoted as follows:

$$
\begin{aligned}
\text { PCi/UNIT OF SAMPLE } & =\frac{(p C i \text { of 243)(241 nents) }}{243 \mathrm{cnts}} \\
\text { ERROR } & =1.96 \sqrt{\mathrm{gcnts}+\text { bkg }} \times \frac{p C i \text { of } 243}{\text { nents of } 243} \\
\text { MDA } & =2 \sqrt{\text { okg }} \times \frac{\text { pCi of } 243}{\text { nents of } 243}
\end{aligned}
$$

SITE

ANALYST

DATE
gatCH No.

REVEWED BY DATE 
PLUTONIUM ISOTOPIC CALCULATION SHEET

\begin{tabular}{|c|c|c|c|c|c|c|c|c|c|}
\hline \multirow[t]{2}{*}{ SAMPLE LD. } & \multirow[b]{2}{*}{236} & \multirow[b]{2}{*}{$239-240$} & \multirow[b]{2}{*}{238} & \multirow[b]{2}{*}{236} & \multirow[b]{2}{*}{$|239-240|$} & \multirow[b]{2}{*}{238} & \multirow[b]{2}{*}{236} & \multirow[b]{2}{*}{$239-240$} & \multirow[b]{2}{*}{238} \\
\hline & & & & & & & & & \\
\hline \multicolumn{10}{|l|}{ GROSS COUNTS } \\
\hline \multicolumn{10}{|l|}{ BACKGROUND } \\
\hline \multicolumn{10}{|l|}{ NET COUNTS } \\
\hline \multicolumn{10}{|c|}{$\mathrm{ml} / \mathrm{pCi}$ OF 236 AODED } \\
\hline \multicolumn{10}{|c|}{ SAMPLE-GUANTITY: } \\
\hline \multicolumn{10}{|c|}{$\begin{array}{l}\text { SAMPLE-OUANTITY: } \\
\text { ASHED ( )G }\end{array}$} \\
\hline \multicolumn{10}{|l|}{$\begin{array}{l}\text { ASHED ( ) } \\
\text { TIME }\end{array}$} \\
\hline \multicolumn{10}{|c|}{ ACTIVITY IN PCI/UNIT } \\
\hline \multicolumn{10}{|c|}{ ERROR IN OCI/UNIT } \\
\hline MOA IN DCI/UNIT & & & & & & & & & \\
\hline
\end{tabular}

WAS COUNTED

Activity of individual isotopes is calculated using a direct ratio of the counts and octivity of Pu-236 added to each somple. and counts of eoch isotopic region of interest.

For example, $P u-238$ would be colculated os follows:

$$
\begin{aligned}
\text { PCi/UNIT OF SAMPLE } & =\frac{(P C i \text { of 236) (238 nents) }}{236 \mathrm{cnts}} \\
\text { ERROR } & =1.96 \sqrt{\mathrm{gCntg}+\text { bkg }} \times \frac{\text { PCi of } 236}{\mathrm{nents} \text { of } 236} \\
\text { MOA } & =4.66 \sqrt{\mathrm{bkg}} \times \frac{\text { pCi of } 236}{\mathrm{ncnts} \text { of } 236}
\end{aligned}
$$

"USE VOLUME (L) OR ORY WEIGHT (C) FOR ALL CALCULATIONS UNLESS DIRECTED OTHERWISE.

STOH/AMT

SITE

BATCH NO.

ANALYST

REVEWED BY

DATE

DATE

LAB $3(2-25-3 i$ 


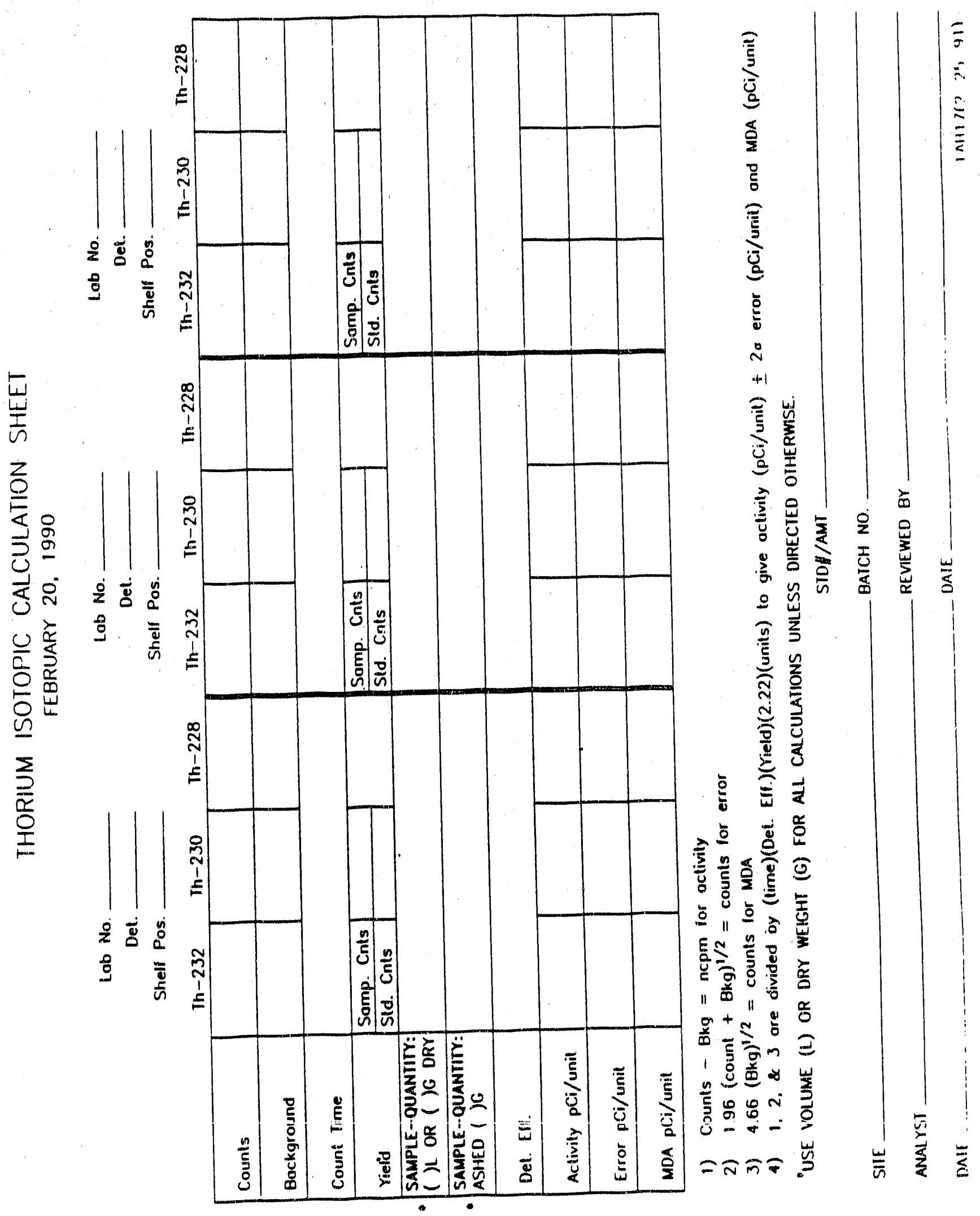


URANIUM ISOTOPIC CALCULATION SHEET

\begin{tabular}{|c|c|c|c|c|c|c|c|c|c|c|c|c|}
\hline \multirow[t]{2}{*}{ SAMPLE 1.0. } & \multirow[b]{2}{*}{238} & \multirow[b]{2}{*}{235} & \multirow[b]{2}{*}{234} & \multirow[b]{2}{*}{232} & \multirow[b]{2}{*}{238} & \multirow[b]{2}{*}{235} & \multirow[b]{2}{*}{234} & \multirow[b]{2}{*}{232} & \multirow[b]{2}{*}{238} & \multirow[b]{2}{*}{235} & \multirow[b]{2}{*}{234} & \multirow[b]{2}{*}{232} \\
\hline & & & & & & & & & & & & \\
\hline \multicolumn{13}{|l|}{ GCNTS } \\
\hline \multicolumn{13}{|l|}{ GCNIS } \\
\hline NCNTS & & & & & & & & & & & & \\
\hline \multicolumn{13}{|l|}{$\begin{array}{l}P C 1 \text { OF } 232 \\
\text { ADOED }\end{array}$} \\
\hline \multicolumn{13}{|c|}{$\begin{array}{l}\text { SAMPLE-QUANTITY: } \\
(x \text { OR ( )G ORY }\end{array}$} \\
\hline \multicolumn{13}{|c|}{$\begin{array}{l}() L \text { OR ( )G DRY } \\
\text { SAMPLE-OUANTITYi } \\
\text { ASHED ( )C }\end{array}$} \\
\hline \multicolumn{13}{|c|}{\begin{tabular}{|l|} 
ASHED ( $) 6$ \\
TIME
\end{tabular}} \\
\hline \multicolumn{13}{|l|}{$\begin{array}{l}\text { ACTIVITY } \\
\text { IN DCi/UNIT }\end{array}$} \\
\hline \multicolumn{13}{|l|}{$\begin{array}{l}\text { ERROR } \\
\text { IN DCi/UNIT }\end{array}$} \\
\hline $\begin{array}{l}\text { MDA } \\
\text { IN PCi/UNIT }\end{array}$ & & & & & & & & & & & & \\
\hline
\end{tabular}

ACTIVITY OF INDIVIDUAL ISOTOPES IS CALCULATED USING A DIRECT RATIO OF THE COUNTS AND ACTNITY OF U-232 ADOED TO EACH SAMPLE, ANO THE
COUNTS OF EACH ISOTOPIC REGION OF INTEREST. FOR EXAMPLE. U-238 WOULO BE CALCULATED AS FOLLOWS:

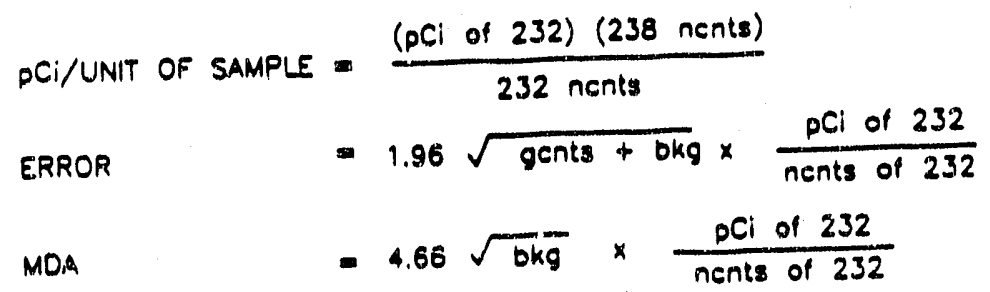

ITHE calculations for U-235 must inClude the alPHa agundance.

-USE VOLUME (L) OR ORY WEIGHT (G) FOR ALL CALCULATIONS UNLESS OIRECTED OTHERWISE. STOH/AMT

SITE BATCH NO.

ANALYST REVIEWED BY DATE DATE 
Contract No. W-7405-eng-26

STRONTIUM-9O DETERMINATIONS BY CERENKOY RADIATION COUNTING FOR WELL MONITORING AT OAK RIDGE NATIONAL LABORATORY

Ingvar L. Larsen

ENVIRONMENTAL SCIENCES DIVISION

Publication No. 1701

NUCLEAR WASTE PROGRAMS

(Activity No. AR 0515150 ; ONL-WLOO9)

NOTICE This documant confuins information of pretiminary naturn. It is subject to revision or correction and therefore does not represent a final repore.

Date Published: June 1981

OAK RIDGE NATIONAL LABORATORY

Oak Ridge, Tennessee 37830

operated by

UNION CARBIDE CORPORATION

for the

DEPARTMENT OF ENERGY 


\begin{abstract}
ACKNOWLEDGMENT
Appreciation is expressed to those individuals who contributed ideas and suggestions concerning this report; they include John Beauchamp, Brian Spalding, Sam Reynolds, and Tom Scott.
\end{abstract}




\section{ABSTRACT}

LARSEN, Ingvar L. 1981. Strontium-90 determinations by Cerenkov radiation counting for well monitoring at Oak Ridge National Laboratory. ORNL/TM-7760. Oak Ridge, Tennessee. 26 pp.

A rapid method for determination of ${ }^{90} \mathrm{Sr}$ (28.8 y half-life) via its daughter ${ }^{90} y(64.1 \mathrm{~h}$ half-life) in aqueous samples from the solid low-level radioactive waste disposal areas at the Oak Ridge National Laboratory (ORNL) utilized the principle of direct Cerenkov radiation counting. This technique is applicable for beta particles exhibiting maximum energles greater than $0.42 \mathrm{pJ}$ (263 keV) in aqueous solution. In the presence of other contributing energetic beta emitters the technique may serve as a useful screening method to distinguish low activity samples from more moderate or higher activity concentrations.

A comparison between analys is of ${ }^{90} \mathrm{Sr}$ by Cerenkov radiation counting and by standard wet chemical separation techniques indicated a high degree of correlation and excellent agreement.

The detection limit for a $20-\mathrm{mL}$ sample under the prescribed experimental conditions and a counting interval of $20 \mathrm{~min}$ was approximately $0.2 \mathrm{dpm} / \mathrm{mL}(0.1 \mathrm{pCi} / \mathrm{mL})$. 


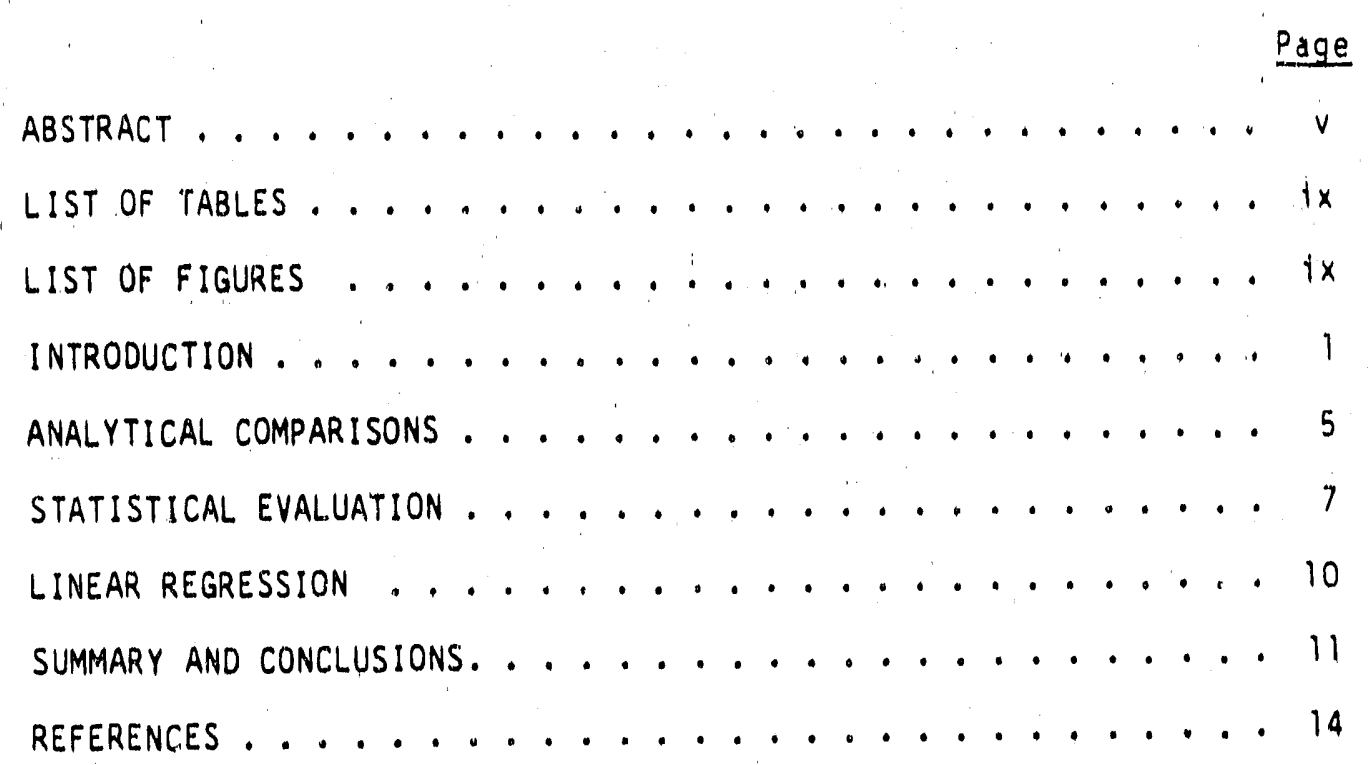


la Effects of $13 z_{s}$ and $60_{\text {Co }}$ on ${ }^{90} \mathrm{Sr}\left({ }^{90} \mathrm{Y}\right)$ determinar. tions by Cerenkov radiation counting ......... 3

10 Decay properties of selected radionuclides ....... 3

2. Comparison between ${ }^{90} \mathrm{Sr}$ determinations by the method of Cerenkov radiation counting and standard analyt. ica) methodology (sample concentrations arranged in descending order $)(\mathrm{dpm} / \mathrm{mL}) \ldots 6$

3 Summary of analytical conditions for Cerenkov radiation counting and chemical separation techniques for

\section{LIST OF FIGURES}

Figure

1 A plot of the residuals labserved value minus predicted value) for linear regression of Cerenkov radiation counting ogainst analytical determinations...? 


\section{INTRODUCTION}

Water samples collected from monitoring wells located within and near the solid lowmlevel radioactive waste disposal areas at Oaic Ridge National haboratory (ORNL) have been routinely analyzed for radioactivity. These analyses include gross alpha, gross beta, ${ }^{90} \mathrm{Sr}$, and ${ }^{3} \mathrm{H}$ detarminations and gamia-spectrometric scans for such radionuclides as ${ }^{60} \mathrm{CO}$ and ${ }^{137} \mathrm{Cs}$. Several hundred samples collected quarterly produced an analytical backlog in the ${ }^{90} \mathrm{Sr}$ measurements that frequently prevented rapid assay and caused corresponding delays in experimental work. This delay was attributed to the low priarity status assigned to these samples; other ORNL investigations of ten took precedence.

The purpose of this report is to demonstrate the feasibility of using cerenkov radiation counting to evaluate ${ }^{90} \mathrm{Sr}$ concentration levels in aqueous samples wilected from within and near the nuclear waste disposal areas at ORNL.

The established methodology for ${ }^{90} \mathrm{Sr}$ determination in aqueous samples at. ORNL involves chemical separation and therefore the use of skilled technicians to perform the analyses. The process requires the addition of strontium carrier to the sample, its precipitation as the insoluble carbonate, and its separation from calcium and magnesium by nitrate preciptation in fuming nitric acid, followed by acetone washes. Further purification is accomplished by removing impurities with mixed-rare-earth hydroxide scavenging and by removing barium as the chromate. Final purification is made by precipltating strontium as the oxalate, which is then beta counted using a low background beta 
ORNL/TM-7760

counter ( $T$. Scott, 1980, Analytical Chemistry Oivision, ORNL, personal communication: ORNL Master Analytical Manual, 1957). This sertes of treatments, which was requited for accurate ${ }^{90} \mathrm{Sr}$ determinations, contributed apprectably to the delay in the analysis. In an attempt to alleviate the ${ }^{90} \mathrm{Sr}$ analys is backlog and to more rapldiy assess ${ }^{90} \mathrm{Sr}$ concentrations, the feasfbllity of using direct Cerenkov radiation counting techniques (Haberer 1966, Ross 1969, Carmon 1979) was investygated. Cerenkov radiation counting is applicable for beta particles in aqueaus solution exhibiting maximum energies greater than $0.042 \mathrm{pJ}$ (0.263 MeV) (Ross 1969). Strontium-90 (28.8 y half-life) exhibits an intermediate beta energy maximum $[0.087 \mathrm{pJ}(0.546 \mathrm{MeV})]$, while its daughter $90_{Y}(64.1 \mathrm{~h}$ half-lffe) has a high maximum beta energy (2.28 MeV), which factlitates, its determination by Cerenkov radiation counting.

Limitations occur in application of Cerenkov radiation counting for ${ }^{90} \mathrm{Sr}$. Cerenkov radiation counting cannot resolve mixtures containing beta emitters of similar maximum energles. The use of electronic discriminators or "window settings" may be necessary to eliminate interference from low-energy beta emitters. In the presence of beta particles that have similar energy but arise from different nuclides, a biased signal may result.

The aqueous samples from the monitoring wells may contain, in addition to any ${ }^{90} \mathrm{Sr}$, other artificial radionuclides of medium half-life such as ${ }^{137} \mathrm{Cs}$ and ${ }^{60} \mathrm{Co}$. The presence of these radionuclides in samples and their effects on cerenkov radiation counting for $90_{Y}$ determinations are illustrated in Table la. Approximately 


\section{C.45}

Table 1a. Effects of $13 \% \mathrm{Cs}$ and $60 \mathrm{Co}$ on $90 \mathrm{Sr}$ (90Y) determinations oy cerenkor radiation

\begin{tabular}{|c|c|c|c|c|c|c|c|}
\hline \multirow[t]{2}{*}{ Radtonuclides } & \multicolumn{3}{|c|}{$\begin{array}{l}\text { Activity } \\
\text { added } \\
\text { (dpm) }\end{array}$} & $\begin{array}{c}\text { Average net } \\
\text { count rate } \\
\text { (com) }\end{array}$ & \multicolumn{2}{|c|}{$\begin{array}{l}\text { Calculated } \\
90 \text { Sr activity } \\
\text { (dom) }\end{array}$} & $\begin{array}{l}\text { Relative contributlons } \\
\text { from } 137 \mathrm{Cs} \text { and } / \mathrm{ar}{ }^{60} \mathrm{Co} \\
\frac{90 \mathrm{Sr} \text { colc. } 90_{S \mathrm{Sr} \text { added }}}{90_{S r} \text { added }}\end{array}$ \\
\hline & 1037 & \pm & $1 \%$ & $385 \pm 9$ & 1026 & \pm 25 & $\cdots$ \\
\hline${ }^{137} \mathrm{Cs}$ & 991 & \pm & $13^{\prime}$ & $13.9 \pm 0.3$ & 37.0 & \pm 0.8 & $\cdots$ \\
\hline${ }^{60} \mathrm{Co}$ & 965 & \pm & 12 & $45.8 \pm 1.9$ & 122 & \pm 5 & $\cdots$ \\
\hline${ }^{137} \mathrm{Cs}+{ }^{60} \mathrm{CO}$ & 991 & + & 965 & $5 \% .3 \pm 1.1$ & 153 & \pm 3 & $\cdots$ \\
\hline${ }^{137} \mathrm{Cs}+{ }^{90} \mathrm{Sr}$ & 991 & + & 1037 & \pm 8 & 1063 & \pm 16 & $2.5 x$ \\
\hline${ }^{60} \mathrm{Co}+{ }^{90} \mathrm{sr}$ & & $\phi$ & 1037 & \pm 1 & 1138 & \pm 3 & 9.78 \\
\hline${ }^{137} \mathrm{cs}+{ }^{60} \mathrm{co}_{\mathrm{O}}+{ }^{90} \mathrm{sr}$ & $991+$ & 965 & +1037 & $438 \pm 6$ & 1167 & \pm 15 & $12.5 x$ \\
\hline
\end{tabular}

Instrument settings as follows (Packard irlcarb liauld scintillation counter model 3225 coincident mode operation):

$\begin{array}{ll} & \\ \text { Oiscriminator window settings: } & 325-1000 \\ \text { High voltage: } & 1814 \pm 50 \mathrm{~V} \\ \text { Amplifler gain: } & 48 x \\ \text { Sample volume: } & 20 \mathrm{ml},\end{array}$

aAll values are +1 so

oAverage and So of duplicate samples.

Table 10. Decay properties of selected radionuclides

Max. Comoton

electron energy MeV b

Halfolife $\quad B-\max$. MeV ${ }^{d} \mathrm{MeV}^{d}$ (neglecting oinding energy)

\begin{tabular}{lllll}
\hline $60 \mathrm{Co}$ & 5.2 y & 0.318 & 1.33 & 1.12 \\
& & & 1.17 & 0.96 \\
$137 \mathrm{Cs}$ & 30.2 y & $0.518(948)$ & 0.662 & 0.48 \\
& & $1.18(68)$ & & \\
$90 \mathrm{Sr}$ & $28.8 \mathrm{y}$ & 0.546 & & \\
$90 \mathrm{y}$ & $64.1 \mathrm{n}$ & 2.28 & & \\
\hline
\end{tabular}

a) $\mathrm{MeV}=0.1602 \mathrm{pJ}$.

DCalculated from Eq. 4.2 in National Counctl on Radiation Protection and Measurement, 1978,

A Handbook of Radioactivity Measurements and Procedures, NCRP Report No. 58, Wasnington, D.C. 
equal activities of ${ }^{137} \mathrm{Cs}$ and/or ${ }^{60} \mathrm{Co}_{0}$ were added to test solutions containing ${ }^{90} \mathrm{Sr}$. Under the experimental conditions, the presence of ${ }^{137} \mathrm{Cs}$ contributed less than $3 \%$ to the ${ }^{90} \mathrm{Sr}$ determination, whereas ${ }^{60}$ Co contributed approximately $10 \%$ to the calculated ${ }^{90} \mathrm{Sr}$ content. Although, ${ }^{60} \mathrm{Co}$ has a lower maximum beta energy $[0.05 \mathrm{pJ}(0.318 \mathrm{MeV})]$ than ${ }^{137} \mathrm{Cs}[0.082$ pJ $(0.514 \mathrm{MeV}$ max $)]$, its influence may be attributed to the more energetic compton electrons (Table ib). Typically, ${ }^{60}$ Co concentrations are less than $1 \mathrm{dpm} / \mathrm{mL}$ for the monitoring wells, a) though occasionally higher concentrations are encountered. When necessary, corrections for ${ }^{60}$ Co interferences can be made following a gamma spectrometric analysis (Reynolds and Eldridge 1980). Faise positive signals arising from other contributing radionuclides can be tolerated in screening applications; and false negative signals are not possible except under conditions of extreme color quenching.

Additional limits to Cerenkov radiation counting can be caused by color quenching or by suspended material, both of which interfere with light transmission from the sample to the detector. These deficiencies, however, can be overcome when necessary by using the internal standard additions method which involves adding a minuscule known amount of ${ }^{90} \mathrm{Sr}\left({ }^{90} \mathrm{Y}\right)$ to the solution after an initial count and recounting to determine an efficiency factor for the quenched solution. Samples collected from monitoring wells are filtered prior to analysis, minimizing suspended particulate effects. The probability of color quenching can be assessed by visual inspection: samples that appear colored should be checked for counting efficiency via the internal standard methods. 
ANALYTICAL COMPARISONS

A comparison between the standard ORNL chemical separation techrique and that of the Cerenkov radiation counting method was performed on 42 samples from ORNL waste disposal areas. Samples for Cerenkov radiation counting consisted of $20 \mathrm{~mL}$ of well water (subsampled from a large container after allowing sufficient time to reach secular equilibrium) directly pipetted into plastic liquid scintillation vials without any scintillation cocktail and counted for $20 \mathrm{~min}$. A blank composed of $20 \mathrm{~mL}$ of distilled water was used to correct for instrumental background contributions. The net couric rates of the samples were then converted to disintegrations per minute using a counting efficiency calculated from a standard of ${ }^{90} \mathrm{Sr}\left({ }^{90} \gamma\right.$ in secular equilibrium) of the same volume. The concentration range of the 42 samples spanned four orders of magnitude. The results of this comparison of the two methods are given in Table 2.

The data reported in Table 2 are expressed to the nearest $0.1 \mathrm{dpm}$ unless concentrations exceeding $99.9 \mathrm{dpm}$ are encountered, in which case the values are rounded to three significant figures. The Cerenkov counting efficiency in this analys is was approximately $48 \%$. The detection limit under the specified counting condition, which is defined as the lowest concentration that can be present in the sample in order to report a positive value $95 \%$ of the time after correcting for background interferences (Aitshuler and Pzsternack 1963, Pasternack and Harley 1971), is approximately $0.2 \mathrm{dpm} / \mathrm{mL}$. The reported uncertainty terms are counting errors of one stantuard deviation. 


\section{C-48}

ORNL/TM-7760

Table 2. Comparison between $90 \mathrm{Sr}$ determinations by the method of Cerenkov radiation counting and standard analytical methodology (sample concentrations arranged in descending order $)(\mathrm{dpm} / \mathrm{ml})$

\begin{tabular}{|c|c|c|c|c|c|c|c|}
\hline $\begin{array}{l}\text { Sample } \\
\text { number }\end{array}$ & Cerenkov & $\pm S D$ & Analytical & \pm 50 & $\begin{array}{l}\text { Cerenkov } \\
\text { andytical }\end{array}$ & $\begin{array}{l}\text { Logarithm } \\
\text { of ratio } \\
\end{array}$ & $\begin{array}{c}\text { Cerenkov minus } \\
\text { andiytical }\end{array}$ \\
\hline $\begin{array}{l}1 \\
2 \\
3 \\
4 \\
5 \\
6 \\
7 \\
8 \\
9 \\
10 \\
11 \\
12 \\
13 \\
14 \\
15 \\
16 \\
17 \\
18 \\
19 \\
20 \\
21 \\
22 \\
23 \\
24 \\
25 \\
26 \\
27 \\
28 \\
29 \\
30 \\
31 \\
32 \\
33 \\
34 \\
35 \\
36 \\
37 \\
38 \\
39 \\
40 \\
41 \\
42\end{array}$ & $\begin{array}{r}2395.0 \\
824.0 \\
715.0 \\
335.0 \\
215.0 \\
204.0 \\
158.0 \\
124.0 \\
105.0 \\
101.0 \\
77.7 \\
67.7 \\
66.4 \\
57.5 \\
56.4 \\
47.5 \\
35.3 \\
32.2 \\
31.3 \\
30.9 \\
28.2 \\
24.9 \\
8.0 \\
7.5 \\
0.9 \\
4.9 \\
4.7 \\
3.1 \\
2.9 \\
2.9 \\
2.7 \\
1.8 \\
1.8 \\
1.6 \\
1.6 \\
1.3 \\
0.9 \\
0.9 \\
0.4 \\
0.4 \\
0.2 \\
0.2 \\
\end{array}$ & $\begin{array}{l}3.40 \\
2.10 \\
1.90 \\
1.20 \\
1.00 \\
1.00 \\
0.90 \\
1.30 \\
0.70 \\
0.70 \\
0.100 \\
1.10 \\
1.10 \\
0.50 \\
0.50 \\
0.50 \\
0.40 \\
0.40 \\
0.40 \\
0.40 \\
0.40 \\
0.40 \\
0.20 \\
0.20 \\
0.20 \\
0.20 \\
0.20 \\
0.15 \\
0.15 \\
0.15 \\
0.10 \\
0.10 \\
0.10 \\
0.10 \\
0.10 \\
0.10 \\
0.10 \\
0.10 \\
0.10 \\
0.10 \\
0.10 \\
0.10\end{array}$ & $\begin{array}{r}2370.00 \\
910.00 \\
720.00 \\
338.00 \\
197.00 \\
204.00 \\
162.00 \\
131.00 \\
106.00 \\
97.00 \\
84.00 \\
62.00 \\
71.00 \\
54.00 \\
55.00 \\
46.00 \\
34.00 \\
31.00 \\
31.00 \\
29.00 \\
26.00 \\
24.00 \\
6.60 \\
6.90 \\
6.70 \\
2.20 \\
4.60 \\
2.60 \\
3.00 \\
2.90 \\
2.80 \\
1.80 \\
1.80 \\
1.60 \\
1.40 \\
1.70 \\
1.30 \\
0.82 \\
0.54 \\
0.41 \\
0.32 \\
0.26\end{array}$ & $\begin{array}{l}30.00 \\
5.00 \\
5.00 \\
3.00 \\
2.00 \\
2.50 \\
2.00 \\
2.00 \\
1.00 \\
1.00 \\
1.00 \\
0.50 \\
0.50 \\
0.50 \\
0.50 \\
0.50 \\
0.50 \\
0.50 \\
0.50 \\
0.50 \\
0.50 \\
0.50 \\
0.20 \\
0.15 \\
0.10 \\
0.05 \\
0.10 \\
0.10 \\
0.15 \\
0.05 \\
0.10 \\
0.05 \\
0.05 \\
0.05 \\
0.05 \\
0.05 \\
0.05 \\
0.05 \\
0.02 \\
0.04 \\
0.04 \\
0.01\end{array}$ & $\begin{array}{l}1.01055 \\
0.90549 \\
0.99306 \\
0.99112 \\
1.09137 \\
1.00000 \\
0.97531 \\
0.94656 \\
0.99057 \\
1.04124 \\
0.92500 \\
1.09194 \\
0.93521 \\
1.06481 \\
1.02545 \\
1.03261 \\
1.03824 \\
1.03871 \\
1.00968 \\
1.06552 \\
1.08462 \\
1.03750 \\
1.21212 \\
1.08696 \\
1.02985 \\
2.22727 \\
1.02174 \\
1.19231 \\
0.96667 \\
1.00000 \\
0.96429 \\
1.00000 \\
1.00000 \\
1.00000 \\
1.14286 \\
0.76471 \\
0.69231 \\
1.09756 \\
0.74074 \\
0.97561 \\
0.62500 \\
0.76923 \\
1.01914 \\
0.22570 \\
0.03483\end{array}$ & $\begin{array}{r}0.01049 \\
-0.09927 \\
-0.00697 \\
-0.00892 \\
0.08743 \\
0.00000 \\
-0.02500 \\
-0.05492 \\
-0.00948 \\
0.04041 \\
-0.07796 \\
0.08795 \\
0.06698 \\
0.06280 \\
0.02514 \\
0.03209 \\
0.03752 \\
0.03798 \\
0.00963 \\
0.06346 \\
0.08123 \\
0.03681 \\
0.19237 \\
0.08338 \\
0.02941 \\
0.80078 \\
0.02151 \\
0.17589 \\
-0.03390 \\
0.00000 \\
-0.03637 \\
0.00000 \\
0.00000 \\
0.00000 \\
0.13353 \\
-0.26826 \\
-0.36772 \\
0.09309 \\
-0.30010 \\
-0.02469 \\
0.47000 \\
-0.26236 \\
0.000717 \\
0.184520 \\
0.028472\end{array}$ & $\begin{array}{r}25.00 \\
-86.00 \\
-5.00 \\
-3.00 \\
18.00 \\
0.00 \\
-4.00 \\
-7.00 \\
-1.00 \\
4.00 \\
-6.30 \\
5.70 \\
-4.60 \\
3.50 \\
1.40 \\
1.50 \\
1.30 \\
1.20 \\
0.30 \\
1.90 \\
2.20 \\
0.90 \\
1.40 \\
0.60 \\
0.20 \\
2.70 \\
0.10 \\
0.50 \\
-0.10 \\
0.00 \\
0\end{array}$ \\
\hline
\end{tabular}

$1 \mathrm{~Bq}=1 \mathrm{dps}=60 \mathrm{dpm}=27.03 \mathrm{pCq}$ 
Concentrations reported in Table 2 for analytical radiochemical results refer to the determinations performed by the standard chemical separation procedures previously described. These determinations were made on ${ }^{90} \mathrm{Sr}$ before ingrowth of activity of its daughter $90_{Y}$. Sample counting time is nominaliy $20 \mathrm{~min}$. Detection limits are approximately $1 \mathrm{dpm}$ of ${ }^{90} \mathrm{Sr}$ per sample, which corresponds to approximately $0.002 \mathrm{dpm} / \mathrm{mL}$ for a $500 \mathrm{~mL}$ sample. The data are usually reported with two significant figures for values less than $100 \mathrm{dpm} / \mathrm{mL}$ and with three significant figures for concentrations exceeding $100 \mathrm{dpm} / \mathrm{mL}$. The reported uncertainty terms are counting errors of one standard deviation. (Table 3 summarizes the analytical conditions for the two methods of analysis.)

The precision terms reported in Table 2 are not expected to be identical because they are based on "counting statistics" and are, therefore, functions of the sample size, concentration, counting efficiency, and counting time of each particular analytical technique. Rather, these terms reflect the expected reproducibility for a given sample and the experimental conditions under which it was determined.

\section{STATISTICAL EVALUATION}

The paired observations can be statistically evaluated using a Student-t test to determine if a significant difference exists between the two methods for the paired sample determinations. Several options in using this test are available and include:

(1) testing the overall mean of the differences of each pair for significance from zero; 
Table 3. Sumiary of the andytical gonditions for Cerenkor radiation counting and chemical separation techniques for $98 \mathrm{Sr}$ determination

\begin{tabular}{|c|c|c|}
\hline & $\begin{array}{c}\text { Cerenkov radiation } \\
\text { counting }\end{array}$ & $\begin{array}{c}\text { Chemtcal separation } \\
\text { technt ques }\end{array}$ \\
\hline Instrument & Liquid scintillation counter & $\begin{array}{l}\text { Cas flow low-background } \\
\text { beta counter }\end{array}$ \\
\hline Procedures & $\begin{array}{l}\text { Direct counting } \\
\text { (coinc ident mode) }\end{array}$ & Chemical precipitation \\
\hline Sample contalner & $\begin{array}{l}25-m 1 \text { plastic } \\
\text { scintiliation vial }\end{array}$ & $\begin{array}{l}\text { Card mounted filter } \\
\text { paper }\end{array}$ \\
\hline $\begin{array}{l}\text { Semple volume } \\
\text { required }\end{array}$ & $10-20 \mathrm{mls}$ & $100-500 \mathrm{~m} / \mathrm{s}$ \\
\hline $\begin{array}{l}\text { Sample preparation } \\
\text { time }\end{array}$ & $25 \mathrm{~m} / \mathrm{n}$ & $28 n$ \\
\hline Counting time & 20 min & $20 \mathrm{~min}$ \\
\hline $\begin{array}{l}\text { Number of samples } \\
\text { processed per day }\end{array}$ & .70 & 224 \\
\hline Background CPM & $25-10$ & 20.5 \\
\hline Detection IImt & $20.2 \mathrm{dpm} / \mathrm{ml}$ & $1 \mathrm{dpm} / \mathrm{sanple}(0.01-0.002 \mathrm{dpm} / \mathrm{ml})$ \\
\hline Detection effictency & $240-508$ & $225 x$ \\
\hline
\end{tabular}


(2) testing the overall ORNL/TM-7760 cance from unity; or

(3) testing the overall average of the logarithm of the ratio of each data pair for significance from zero (which is the same as testing the average difference between logarithms of the data pairs).

Because the ranges in concentrations covered four orders of magnitude, and because a greater absolute difference is expected between larger quantities than between smaller quantities, the test was applied to logarithms of the ratio of the paired observations. This treatment tends to "normalize" any extreme magnitudes occurring in differences over low to high concentrations.

In using the Student-t test, the differences in individual pairs are assumed to be distributed about some mean that represents the average of the difference in the effects of the two treatments over the population of which these pairs are considered random samples (Snedecor and Cochran 1967). The differences are assumed to be random, normally and independently distributed, with a population mean of zero. Using coded index values (Barnett 1975), the logarithms of the ratios of the paired observations can be shown to approximately follow these assumptions.

The Student-t test is given as follows (Snedecor and Cochran 1967):

$$
t=\frac{D-0}{S_{D}}, S_{D}=\frac{S_{D}}{\sqrt{n}}, S_{D}=\sqrt{\frac{\Sigma(D i-O)^{2}}{n-1}} .
$$


where

$D_{i} \quad=$ logarithm of the ratio of the data pairs,

$\delta=$ sample average of the ratios,

$n \quad$ = number of data pairs, and

$n-1$ = degrees of freedom $\left(d . f_{.}\right)$.

From the mean and standard error of the logarithm of the ratio of the concentration by the two methods (Table 2), the calculated t value is

$$
t=\frac{0.000714-0}{0.028472}=0.02508
$$

A comparison of the above calculated $t$ value with tabulated Student-t; values with $41 \mathrm{d.f}$. indicates no significant difference in the ${ }^{90} \mathrm{Sr}$ determinations between the two methods, not only at the $5 \%$ probability level but even it a significance level as large as $40 \%$.

\section{LINEAR REGRESSION}

Linear regression analys is was applied to examine the degree of correlation between the data sets. The Cerenkov radiation values were considered the dependent variable and the results from the standard analytical method were considered the independent variable. The parameters examined included the correlation coefficient, slope, and intercept values. A plot of the residuals (observed dependent value minus the predicted value) was also examiried to verify that the deviations were random. The correlation coefficient $r$ (or $r^{2}$ ) provides a measure of the degree of yariation for which the dependent variable can be 
accounted for by the model. The slope of the regression line describes the change in the dependent variable per unit increase of the independent variable. Ideally a one-to-one correspondence occurs. The intercept provides an indication of bias or systematic error present in the determination of the dependent variable and ideally should be zero in the absence of intrinsic influences.

For the data in Table 2, a high degree of correlation exists with $r^{2}=0.9987$. The slope is $0.9977 \pm 0.0057$ and is not significantly different $(P>0.05)$ from unity, indicating a one-toone correspondence. The intercept value is $-0.7621 \pm 2.3879$ and is not significantly different $(P>0.05)$ from zero, indicating the absence of any predominant systematic bias. A plot of the residuals against the predicted value (Fig. 1) revealed a fairly uniform pattern about zero. As anticipated, a greater absolute difference occurs between the larger concentration of ${ }^{90} \mathrm{Sr}$ than between the smaller concentrations determined by the two methods, and such differences are exemplified in Fig. 1 .

\section{SUMMARY AND CONCLUSIONS}

The determination of ${ }^{90} \mathrm{Sr}$ (via its ${ }^{90} \mathrm{Y}$ daughter) by Cerenkov radiation counting on aqueous samples from monitoring wells within and near the solid low-level radioactive waste disposal areas at ORNL has been found to be highly correlated with standard chemical separations methodology. Comparisons of the two methods using paired concentration daca were highly favorable and indicated no significant difference $(P>0.05)$. A considerable savings in time and expenses can be 

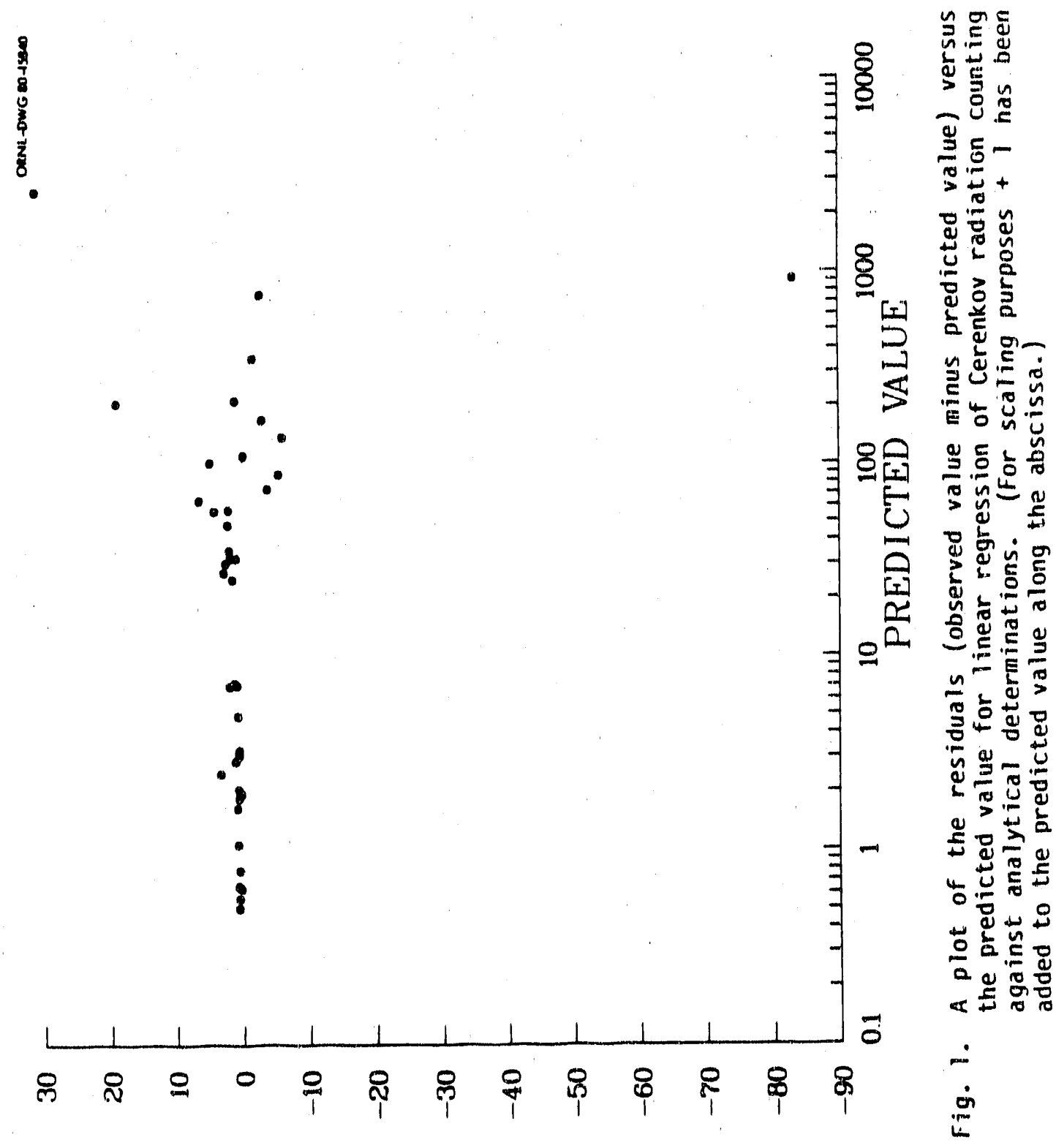


\section{C-55}

achieved by utilizing Cerenkov radiation counting to screen samples that contain little if any ${ }^{90} \mathrm{Sr}$ radioactivity. For example, the analysis of 119 monitoring well samples for ${ }^{90} \mathrm{Sr}$ concentrations by Cerenkov radiation counting revealed that only 33 samples (28\%) contained ${ }^{90} \mathrm{Sr}$ concentrations of $3 \mathrm{pCi} / \mathrm{mL}(6.7 \mathrm{dpm} / \mathrm{mL})$ or greater. These arbltrarily selected samples (33) were inen submitted to the Analytical Chemistry Division for ${ }^{90} \mathrm{Sr}$ determination using standard wet chemical separation methods; this procedure eliminated from the tedious and costly analysis those samples that were low or near zero concentrations. Reductions (w0\%) in both cost and sample processing time were achieved by screening these samples by Cerenkov radiation counting. 


\section{REFERENCES}

Altshuler, B., and B. Pasternack. 1963. Statistical measures of the lower limit of detection of a radioactive counter. Health Phys. $9: 293-298$.

Barnett, V. 1975. Probability plotting methods and order statistics. App 1. Stat. 24(1):95-108.

Carmon, B, 1979. The use of Cerenkov radiation for the assay of radiostrontium in aqueous solutions. Int. J. Appl. Radiat. Isot. $30: 97-99$.

Haberer, K. 1966. Measurement of beta activities in aqueous samples utflizing Cerenkov radiation. Technical Bulletin No. 16. Packard Instrument Co., Inc., Downers Grove, Illinois. $14 \mathrm{pp}$.

Oak Ridge National Laboratory Master Analytical Mannual. 1957. T10-7015, 221082.1 to 2 21082-10. Oak Ridge, Tennessee. Pasternack, B. S., and N. H. Harley. 1971. Detection limits for radionuclides in the analysis of multi-component gamma-ray spectrometer data. Nucl. Instrum. Methods 9(3):533-540.

Reynolds, S. A., and J. S. Eldrige. 1980. Investigations of Cerenkov counting of environmental strontiumm90. pp. 397-405. IN C. T. Peng, O. L. Harrocks, and E. L. Alpen (eds.), International Conference on Liquid jcinitillation Counting: Recent Applications and Developments, Vol. 1: Physical aspects. Academic Press, New York. 
Ross, H. H. 1969. Measurement of B-emitting nuclides using Cerenkov ractiation. Anal. Chem. 41:1260-1265.

Snedecor, G. W., and W. G. Cochran. 1967. Statistical Methods, 6th Edition. Iowa State University Press, Ames, lowa. 593 pp. 
ENVIRONMENTAL SCmences DIVISTON

PROCE JRES
PROCEDURF

PAGE:

1 of 2

REVISION DATE:

TITLE: Techisical Procedure for Callbration of IO and Ce(L) Detection

\subsection{Purpose}

Calibration of sosxial and planar [IG \& Ge(LU)] solid state detectors in Rm $144 \mathrm{~B}$ and $\mathrm{Rm}$ 169. These detecton are used in quantifying low-level gamma-ray emitting ndionuclider.

\section{Equipment}

21 IG and $\mathrm{Ge}(\mathrm{Li})$ solid state coardal detectors and well detector.

2210 solid state planar detector.

\subsection{Procedures}

3.1 Culibration of the conxial detectors is to be accomplimbed using certified gamma-ray standards with traceability to NIST (Notional Institute for Standards and Technology). Une Amessham mixed gamma standards (QCY44, QCY46, or QCY48) or an appropriate substitute if these are not available or does not contain the appropriate energy region. An alternative may be used, but if not traceable to NIST, certificstion by the insuing ageacy must be available.

32 Use an appropriate amount of the standard (dead time should be $10 \%$ or less), and dilute if aecessary with appropriate material (water, acid, or base). A carrier solution similar to the Amershum non-radionctive carrier solution or a suitable alternate should be added when available. Typical activity ranges for courial detectors would be 10 to 100 nanocuries per radionuclide. For well detectors use considerably leas (approximately 1 to $10 \mathrm{nCl}$ per nuclide) because of coincidental summation photopeales.

3.3 For moat analyzes, liquid standards are appropriate for quantification of gammaray mitting radibnuclides contained in soil, sediment, milk, or aqueous solutions (Lenen and Cutstall 1981). For non-metallic containers, dilution of the Ameraham standard and carrier solution with $4 \mathrm{M} \mathrm{HCl}$ as per directions by the vendor is appropriate. If dilution with $4 \mathrm{M} \mathrm{HCl}$ is not appropriate, an aquenus colution of the stapdard in chelating solution (i.e., EDTA or equivalent) stall uned. If preferred or if leaknge from the container presents a problem, a gelling compound (agar, gelatin, pectin, "water grabber") should be used to "solidify" the staudard.

\section{CAUTTON}

This document has not been given final patent clearance and is for internal use only. If this documeat is to be given public release, it must be cleared through the sito Tachnical Information Office which will see that the proper patent and technical information reviews are completed in accordance with Energy Systems Policy 
TITLE: Technical Procodure for Calbratioa of IO and Ge(L) Detoctors

3.4 Count the standard for an appropriate container for an appropriate amount of time to minimize the counting error (coefficient of variation approximately $5 \%$ or less per gamma-line). Typical counting times range from 30 ininutes to 1000 minutes depending on activity of the gamma emitter and desired precision.

3.5 Calibration of the Intrinsic Germanium (IG) planar detectors (low-energy photon detectors) for Pb-210 should be done by using a Pb-210 standard with traceability to NIST or alternate calibration source sisch as uranium ore from the New Brunswick Laboratory (NBL) or other suitable certified material (e.g. Canadian Certified Uranium Ore). In some cases, standard material traceability to NIST may not be avallable. In this case, documentation regarding certification or ausay must be available.

3.6 For otber low energy photen etnitterz, appropriate standards will be used in the appropriate counting container.

3.7 Acceptable criteria for calibration will be based on agreement of crosscheck sumples and/or blind sampies supplied by the EPA, material supplied by NIST (NBS), the International Atomic Energy Agency (LAEA), or other responsible agencies supplyiag crosscheck samples. Doeuments for these materials will be maintained.

3.8 Documentation records for calibration and croscchecks shall be maintained by L. L. Lanen and/or J. D. Mareh, Jr.

\subsection{Refenencos}

I. L. Lamen and N. H. Cutshall. 1981. Direct Determination of Be-7 in Sediment. Earth and Planetary Science Letters. 54:379.384.

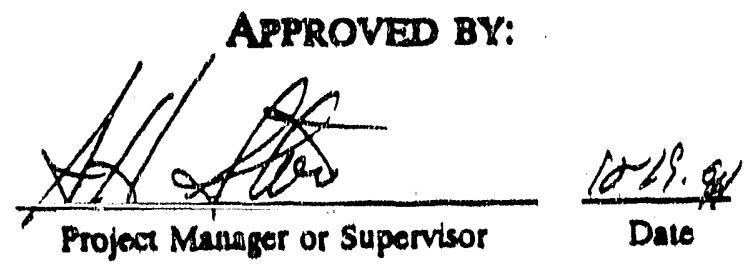


TITLE: Technical Procedure for Openting ND 9900 Gumma-Ray Spectrometer

\subsection{Purpose}

The purpose of this technical procedure is to provide instruction in operating the Nuclear Data 9900 Microproceseor Syztem. Additional details are given in the instrument manual. Onty qualified personnel are allowed to operate the system and includes I. L. Larsen, J. D. Marsh, Jr., Craig Brandh and trainees under their direction.

\section{Equipment}

Nuclear Data 9900 Microprocessor Data Acquisition System

$3 \mathrm{Ge}(\mathrm{Li})$ coarial detectors ( $\# 1, \# 2, \# 3$ )

1 IG (intrinsic Germanium) conxial detector ( $\$ 6)$

1 IG conxial well detector (\#7)

3 IG planar detectors (\#4, \#5, \#8)

\subsection{Procendures}

3.1 Sample containers, sboes, gloves (if worn), etc., must be free of external contamination before entering the counting room.

32 Only sample of low radioactivity sbould be anabyzed in the low-level courting room to avoid possible contamination of the detectors for future gamma-ray analyzis.

33 Each detector is affiliated with a computer sccount on the ND 9900 system. To accese and analyze the data collected by a detector, log on to the appropriate computer sccount by typing USER\#, where * is a number from 1 to 8 which identifies the detector and user (i.e., USER1).

3.4 Wrap the sample in a plastic bag and place on the appropriate detector. Set the counting time by typing PARS DET \# PLVVE = XXXXXX seconds. Record the sample in the log book. Chect the complete sample label by typing PARS DET * SAMPLE= or depreas key PF4 on right side. Change the various label parameters $a$ necewary when prompted.

35 Begin scquisition of data for the detector by depreasing sequentially the ACQ and INTT keys bocated at the top of the keytoard.

\section{CAUTTON}

This document has not been given final patent clearance and is for internal use only. If this document is to be given public release, it must be cleared through the site Texhnical Information Office which will see thut the proper patent and tachnical information reviews are compieied in accordance with Energy Systems Policy. 
ENVIRONMENTAL SCIENCES DIVISION PROCEDURES
PROCEDURE:

PAGE:

2 of 2

REVISION DATE:

TITLE: Technical Procedare for Operating ND 9900 Gamma-Rry Spectromeler

3.6 After sample bas been counted, turn off the acquire mode by again pressing sequentially the ACQ and DNTT keys at top of keyboard. Alternatively if the present PLIVE TIME option is used, the acquistion of counts will terminate when preset time has been achieved.

3.7 Type the command PEAK DET $/$ LIST $=$ TTAX where $X$ is the line printer output file $(0,1$, or 2$)$. A peak search of the gamma spectrum will be performed and results listed to the appropriated line printer.

38 To correct for background type ENBACK/BKGND $=$ BG_DET $. D A T E / L I S T=T T A$, where DATE identifies the background A directory of available background corrections can be obtained by typing DIR BG_DET

39 To obtain a report summarizing the potential radionuclides present, type the command WTMEAN/REPORT $=A C T / L I S T=T T A *$. If queried for the nuclide library, respond with LIBD. To select the appropriate efficiency file. consult the directory EFF_DET*.

3.10 After the analysis is completed, remove the sample from the detector and check label against that on the printout. Erase the displayed spectrum from the monitor by depressing ERASE and INTT keys at top of keyboard.

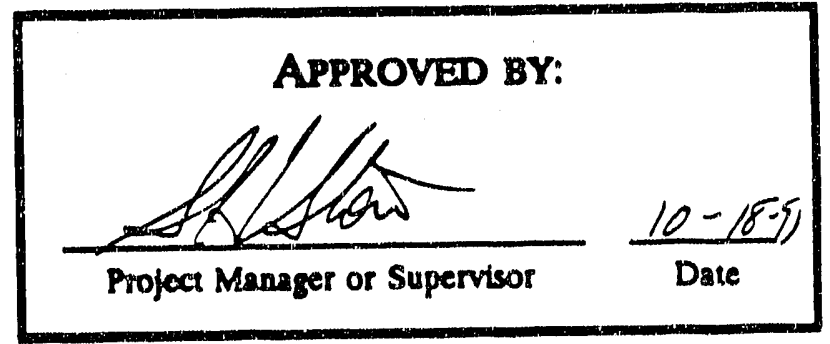


ENVIRONMENTAL SCIENCES DIVISION

PROCEDURE:

PAGE:

TITLE: Technical Procedures for QAOC Activities for Low-Lovel Gamm-Ry Anatyas in Rm 144B

\subsection{Purpoce}

The purpose of this document is to describe the procedure used in the low level counting Room 1448 in Building 1505 and the quality control/quality assurance procedures.

\section{Scope}

These procedures include quality assurance/quality control mechodology for gammagay analysis performed on the detectors in Room 144B.

\subsection{Referencen}

American National Stundard: Calibration and usage of germanium detectors for mensurements of gamma-ray emission of ralionuclidea. American National Standeards Institute, Inc.. Institute of Electrical and Electronics Engineers, Inc.. 345 47th St., New York NY 10017. ANSI N42.141978. April 10, 1978. I.L Larsen and N.HL Cutshall. 1981. Earth and Planetary Science Letters 54:379-384.

\subsection{Requiremeats}

Environmental Sciences Division QA procesdures QA-ES-5-102 requires that all laboratories have laboratory operations minuals that contain or reference the latest approved version of technical procedures, safety procedures, and QAVOC procedures for the work performed in the laboratory.

\subsection{Responsibilitices}

The QA procedure and gamma-gay analysis in Room 1448 will be the responsibility of the laboratory steward (L. L Lanea) and/or his designate (J. D. Miarsh. Jr.). Trainees will be supervised only under the direction of Lanen or Marsh.

\section{Equipment}

\section{Nuclear Data 9900 System}

$623 \mathrm{Ge}(\mathrm{Li})$ solid state covial detectors

631 Gel(IG) solid state coscial detector

\section{CAUTION}

641 Gei(IG) solid state well detector and is for internal use only. If this document is to be givel public release, it must be cleared through the site Technica Information Office which will see that the proper patent and recinnicai iúfomation reviews are completed it accordance with Energy Systems Policy. 


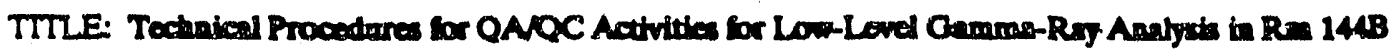

7.0 Procectures

7.1 Once a week count the C-137 EPA spiked elay rample in can geometry on each operational coerial detector and on the well detector. Counting of quality assurance samples should be scheduled to aroid interfering with routine sample andyin Therefore, do not interfere with bong period counts which may be required for some sumplex. Count thin sample for $60-100 \mathrm{~min}$ and record data on log sheet and plot on control chart. For planer detectorn. count the Canadian Uranium Certified Referenoe ore sample BL-1 for $60-100$ minuter Record data on bos abeet and plot co control chert.

72 Check energy calibration by comparing enersy of photopeabs with known energ. This should be done weetly with the exception of ongoing long countr. Record calibration data for each detector in instrumeat performance log book. Aleo, during routine counating if the known energy value for photopeabs deviates by more than $20 \mathrm{Kev}$, then update energs calibration following procedure in instrumentation boot Use Ba-133, CS-137, and Co-60 sources for enersy calibration of the comial detectors and use Pb210, Am-241, Ba-133 sources for the planar detectors.

7.3 For each seriee of sediment samples counted on cosxial detectors, count a NBS (National Bureau of Standards, now NIST National Institute for Standards and Technolony) Certified Refereace Material sample (i.e., NBS 4354 latre exdimeat; NSB 4353 Rocky Flats coil; NBS 4350 river rediment; or IAEA sedimeat) or similer witable material. Compare the measured value with the bockwa value. If the measured value $+1-$ throe times its standard deviation differs from the trown value +1 - three times its standards deviation, (ien do aot equal or overlap) then a reanalyzis should be done. If such diecrepanciea consistently continue, than an investigation and explanation or a recalibration sbould be done. This does not apply to sbort half-life radionuclides (i.e, leat than 1 year) which may docay away over time or to samples at or below the minisium detectable activity.

7.4 Within each series of samplea, a field duplicate sbould be counted if available. In the abresces of a field duplicate, a replicate count of one sample from that serices should be done. It is advisable that at least one duplicate or replicate count be made for each of 20 samplea. If the field duplicates differ by more than three stundards deviations (a deacribed in 7.3 above), than a recount of each duplicate should be performed. Field duplicates may often not agree becauve of sampling variability, and this should be taken into consideration. Redionuclides which bave a short halslife may decay away before reanalysis as 
ENVIRONMENTAL SCLENCES DIVISTION ProceEURES
PROCEDURE:

PAGE: 3 of 4

REVISION DATE:

$10 / 18 / 91$

TITLE: Technical Procedures for QAQC Activities for Low-Level Gamma-Rug A dysis in Ran 144B

variability, and this variability should be taken into consideration. Exceptions to this reanalysis criteria are short-lived radionuclides which may decay before reanalysis or radionuclides near the minimum detestable activity. Replicate counts sbould be treated as in Section 7.3 above. Counting time should be selected in accordance with the activity level preaent, the sample size, and the aumber of sample as well as the discretion of the analyst. Typical counting times may range from a few minutes to overnight or bonger.

75 When possible count the "Nucleus" Cs-137 source each day for 1 minute on the coacial detectors and well detector (det 7 ) and record value on log sheet and plot on control chart. Observe that the value is within the control limits (+1three standard deviations) determined from several ( -20 or more) measurements. If outside these limits, do not use the detector. If repeatedly outside the limits, cetermine the cause if possible. When back in operation. verify the detector's performance before sample anabyis by counting on NBS/NIST reference material or similar suituble material. Do not interfere with any long-term counts in progress. For the planar detectors, count the "Totope Products" Pb-210 source for 120 seconds on detectors 4 and $S$ and 300 seconds and detector 8 . Do not interfere with any ongoing long counts. Compare value with the mean value for several counts and record on log sheet and plot on control chart. If value falls outside the control limits ( + -three standard deviations), recount, and if consistently fails to meet this criterion, dircontinue the use of detector until it can be verified for reliable performance.

7.6 Continue to participate with the EPA in their crosecheck program and report summary of resulis from EPA to the Environmental Sciences QAQC officer.

7.7 Update detector efficiency calibrations when needed. This update includes wewty purchaned detectors or detectors returned from manufacturer for repair. This recalibration should also apply when results from analysis of certified reference materinls consistently deviate from the known value as described in 73.

78 Update detector background by counting a distilled water savis:- 'or at lesst 1000 minutea, preferably over a weekend or over a weekead with an adjoining boliday (ien a Friday or Mondiny boliday). The purpose is 20 reduce the courting error due to beckground subtraction. Do a peat output and store the spectrum in the appropriate detector background ale (i.en, BC,DET\#.DATE The command after running peak is: Move Det\# BG_DET.DATE.

Backeround sbould be updated when appropriate but not to interfere with 
ENVIRONMENTAL SCIENCES DIVISION PROCEDURES
PROCEDURE:

PAGE: 4 of 4

REVISION DATE: $\quad 10 / 18 / 91$

TITLE: Technial Procodures for QANC Activities for Low-Level Gamma-Rry Anatysis in Rm 144B

ongoing long counts. Ideally, but not necessarily rigorously, this should be done at least once a month, if the detector is on line.

79 The QAOC procedures and gamma-say analysis in Room 144B will be the responsibility of the laboratory steward (L. L Larsen) and/or his designate (J. D. Marsh, Jr.). Records of QAVC performance will kept available for inspection. Trainces will be supervised only under the direction of Larsen or Marsh.

\subsection{Reconds}

Records of QANOC performance for the curreat and past two years will be kept available in Room 144B for inspection. This includes certificates of calibration standards, detector performancea, calibration records, background analysis, and crosscheck samples with the EPA, NIST, as well as other agencies.

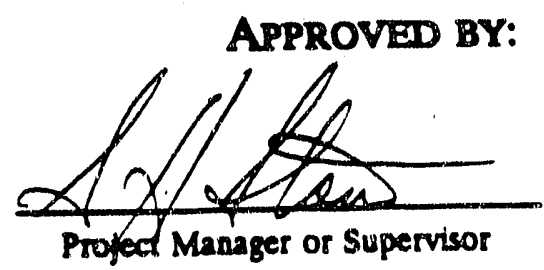


ENYIRONmertal SCIENCES DIVISION PROCEDURES
PROCEDURE:

PAGE: 1 of 3

REVISION DATE: 10/1891

TITLE: Technial Procodura for QANC Aativities in Room 169

1.0 Purpose

To assure quality performance/quality control for radionuclide measurements using equipment in ESD counting room (Room 169).

\section{Scope}

These procedures include quality assurance/quality control methodology for gammaray analysis utilizing the Nuslear Data 6700 microprocessor acquisition system. Additional QAVC procedures for the other nurlear instrumentation in Room 169 will be the responsibility of the primary users (PI's).

\subsection{References}

American National Standard: Calibration and usage of germanium detectors for measurement of gamma-ray emission of radionuclides. American National Stsandards Institute, Inc. Institule of Electrical and Electronics Engineers, Inc., 345 47th St. New York, NY 10017. ANSI N4214-1978. April 10, 1979. Abi, L.L Larsen and N.H. Cutshall. 1981. Earth and Planetary Science Letters 54:379.384.

\subsection{Responsibilitices}

The QA technical procedures for gamma-ray analysis using the Nuclear Data 6700 syrtem will be the responsibility of the laboratory steward (L.L. Larsen) and/or his designate (J.D. Mashh, Jr.). Trainess will be supervired only under the direction of Larien or Marsh or their designate. QA procedures for the instrumentation will be responsibility of the principal investigator.

\subsection{Equipment (operationa)}

\subsection{ND 6700 Gamm System}

5.2 Peckard Tricarb 2000 CA Liquid Scintillation Counter

5.3 Packard Tricarb 4640 Liquid Scintillation Counter

5.4 Peckard Tricarb 460 CD Liquid Scintillation Counter

5.5 Packard Auto Gamm Link Bell Scintillation Spectrometer

5.6 Nuclear Data Link Belt Spectrometer

5.7 ORNL developed Liquid Scintillation Counter

S.8 Harshaw TASC 12 alph, beth gamma gross radiation detector

5.9 Operational procedures and/or manuals are available for each instruments.

\section{CAUTION}




\section{ENVIRONMENTAL SCIENCES DIVISTON}

PROCEDURES
PROCEDURE

PAGE:

2 of 3

REVISION DATE
$10 / 18 / 91$

TITLE: Technical Procedures for QAQC Activities in Room 169

\subsection{Procedures}

Quality assurance in Room 169 is assessed in several ways depending on the type of analysis being performed.

\subsection{ND 6700 Gamm Syatem}

6.1.1 Quality Assurance for the ND 6700 gamma system is performed weekty by counting the EPA spiked clay source on each of the two intrinsic germanium (IG) detectors and plotting results on a control chart. Vaives should fall within +1 . three standard deviation of the mean value determined from previous results.

6.12 A weekly cnergy calibration check will be performed prior to use if the instrument is on line. This is sccomplished by counting a Ba.133 source (81.0 \& $356.0 \mathrm{KeV}), \mathrm{Cs}-137$ source (661.6 Kev) and a Co-60 source (1173. \& $1332 \mathrm{Kev}$ ). Record the data and peak channel for these energy lines in the detector log shees. If out of calibration by more than $2 \mathrm{Kev}$, update energy calibration (see Nuclear Data Manual). If less than 2 Kev, update is not necessary but. may be preferred. Do not interfere with any ongoing long term counts to accomplist this. When the long term count is completed, an energy check can then be done.

6.13 A 1-minute daily detector performance check should be made prior to beginning a new count for that day. The red Cs-137 source is placed on top of an aluminum can on esch detector and counted for 1 minute. The peat area is then printed out and the number recorded along with the date in the detector log book. The value should also be plotted on the detector's control chart, also in the detector log book. If deviations consistently fall above or below the three sigma levels on the chart, do not we the detector for quantification purposen. Take the detector off-line until performance verification is reestablished. Do not interfere with any ongoing long-term counts. A detector check will be done when count is completed and prior to counting new sample.

61.4 Crous-check smmples or "blind samples", which are supplied (typically quarterly) by the EPA through their EMSLLLV Quality Assurance/Quality Control radioanatytical program, will be analyzed to verify calibration and analytical performance. In addition, when applicable, certified reference material supplied by NIST (NBS) or other certified agency should be counted prior to analyzing samplea. Such samples would include NBS 
ENVIRONMENTAL SCAENCES DIVISION Procedures
PROCEDURE:

PAGE: 3 of 3

REVISION DATE:

TITLE: Technical Procederes bor QAOC Activities in Roow 169

SRM 4354 (lake sediment); NBS SRM 4353 (Rocky Flats soil); NBS 4350 (river sedimeat), or IAEA material. Records of analysis shall be kept for impection and verification of performance.

615 Maintenance of the ND 6700 syrtem is performed by qualified penonoel in the I\&C Division at ORNL and/or under a service agreement with Nucleas Data.

\section{Liquid Scintillation Counters}

621 The performance of the liquid scintillation counters is monitored by counting a reference source each time a group of samples is analyzed by a particular user. These sources are generally supplied by the instrumont manufacturer. In addition, ample blank should be counted with esch group of samples. The user will perform his/bers quality assurance/quality control.

622 Maintenance of the liquid scintillation counters will be performed by I \& C or a manufacturers representative when needed.

623 All repairs are performed by qualified I\&C perzonnel.

\section{NAI Linibelt Detector(s)}

63.1 QAOC for the link belt NaI (TI) detectors is to be performed by the particular wer counting hiabers reference source along with a sample blank

632. Repain will be done by qualified personnel from the I \& C division as needed.

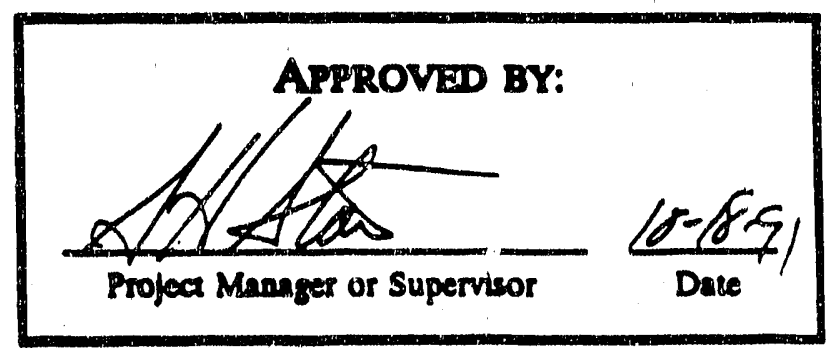




\title{
NONDESTRUCTIVE SAMPLING OF SMALL CARNIVORES FOR GAMMA EMITTING RADIONUCLIDES AND TRACE METALS
}

\author{
prepared by \\ T. L. Ashwood \\ Environmental Biotechnology Section
}

\section{PURPOSE}

To define the actions necessary to obtain hair samples from small carnivores and to prepare those samples for analysis.

\section{SCOPE}

This procedure applies to nondestructive sampling of small carnivores that can be caught in live-traps. The procedure was primarily developed for piscivorous mammals such as raccoons (Procyon lotor) and mink (Mustela vison), but other carnivorous mammals may be sampled by varying the bait, placement of the traps, and dosage of anesthetic. This procedure does not apply to leg-hold traps or snares.

\section{EQUTPMENT}

1. Wire-mesh live-traps.

2. Anchoring stakes for eech trap.

3. Yellow plastic tape for marking traps.

4. Surveyor's flagging for identifying trap locations.

5. Sardines (packed in oil) or other bait.

6. Can opener.

7. Heavy gloves.

8. Rubber gloves.

9. Field clothes appropriate to the trap site.

10. Painter's drop cloth.

11. Syringes ( $\geq 1$ per animal).

12. Ketaset or other approved anesthetic.

13. Tattooing kit (optional).

14. Rubbing alcohol.

15. Paper towels.

16. Plastic bags for hair samples (1 per animal, sandwich bag size).

17. Scissors (keep sharp).

18. Permanent ink marker.

19. Field notebook

20. Stool sample probes (1 per animal).

21. Stool sample containers (1 per animal).

22. Plastic trash bag.

23. First-aid kit to treat possible bite/scratch injuries. 


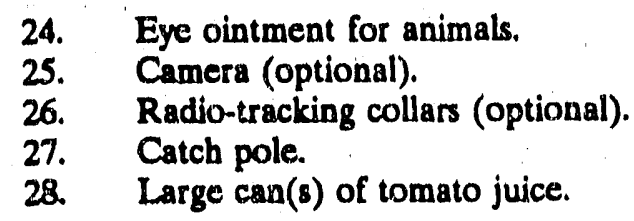

\section{PROCEDURES}

All personnel working with wild carnivores must receive the pre-exposure rabies prophylaxis. This treatment includes three intramuscular shots over a 3-4 week period and boosters as appropriate (to be determined by medical personnel). Personnel who merely observe trapping and sampling activities need not have the pre-exposure series so long as they do not handle either the animals or the traps containing the animals and as long as they maintain a reasonable distance when the animals are uncontrolled or released. Any wound inflicted by an animal should be immediately and thoroughly cleaned. The offending animal should be kept until medical personnel can determine the proper course of action. No one with open cuts or scratches on their hands should handle any wild animal-even if wearing gloves.

A second bealth concern related to handling of wild animals is transfer of parasites, particularty the round worm Baylis asceris', from animals or their scats to bumans. All personnel handling animals, biological samples, or anything that has been in contact with the animal (including the trap) must wear plastic gloves during this handling. As soon as possible after handling any of the preceding items, each individual must thoroughly wash their hands and arms to the elbow with soap and hot water. All clothing that has been in contact with these items must also be laundered, and care should be taken to keep hands, clothing, or any potentially contaminated items away from the face. Eating, drinking, chewing tobacco, smoking, or applying cosmetics are to be avoided while in the field, unless the individual is able to thoroughly wash in the field.

Field research personnel are expected to have whatever training is required for access to the studiy site. Observers who do not have the requisite training may be escorted to the site provided that thcse responsible for access control have approved the practice of escorting visitors and provided all escort requirements are fulfilled.

Personal protective clothing requirements and personnel monitoring procedures vary from site to site. All field researchers and obseivers will comply fully with the established procedures for the study site. As a minimum, field researchers and any observers must wear ORNL provided field clothing (khakis, coveralls, etc.) for study sites on the Oak Ridge Reservation (ORR). Sturdy boots are required for all field researchers, and wet sites may require rubber boots or waders.

\subsection{TRAPPING}

${ }^{9}$ Baylis asceris may be contracted from the scat or fecal material of raccoons and is always fatal when contracted by humans. 


\section{C-73}

All trapping activities must be closely coordinated with the resident Tennessee Wildlife Resources Agent for the ORR. This individual should be notified prior to beginning of the overall program and prior to each specific trapping event. It is strongly advised that a certified veterinarian be consulted about trapping activities and that the veterinarian be available on short notice to assist with trap-related injuries to the animals. In any case, a veterinarian must be involved with the program to provide the anesthetic.

1. Obtain collector's permit from the Enforcement Division of the Tennessee Wildife Resources Agency. To obtain the permit, write to the director of the division and provide a copy of the research proposal and a concise description of the species to be collected and the trapping method. Obtaining a permit usually requires a minimum of two weeks.

2. Mark traps with the name and phone number of the principal investigator in whose name the collector fo permit is issued. Also mark the traps with the permit number and a unique identifying number for each trap. This marking may be in the form of a tag made from yellow plastic tape. Use a permanent ink pen for the tags. Plan on having to retag each trap each day because the animals frequently chew or tear the tags. Weigh a representative empty trap and record the weight in the field logbook for later use in calculating animal weight.

3. Determine the location for each trap based on the terrain to be covered and the objectives of the trapping. For piscivorous mammals, traps should be placed adjacent to streams where there is ready access from the stream to the trap (e.g., do not place the traps at the top of a high, steep bank). Traps may be placed in a gridded array, a linear arrangement along the stream, or randomly as accessibility permits. Put the traps in a location that is out of the way of human traffic. Do not place the traps in a position that may be flooded during a heavy rain.

4. Anchor the trap to the ground with a wooden stake or a length of rebar.

5. Mark the trap location in the field with surveyor's flagging to facilitate finding the trap site again and to provide a basis for repetitive trapping in the same pattern.

6. If possible, place the trups unbaited and securely wired open 2-3 days prior to actual collection to allow the animals to become accustomed to the presence of the trap.

7. Mark the locations of the traps on a map of the area using the unique trap identifying numbers.

8. Late in the afternoon, bait and arm the traps. For piscivorous mammals, use a full can of sardines the first day the trap is baited and $1 / 2$ can on subsequent, consecutive trap days. Place $\sim 1 / 3$ of the bait at the rear of the trap, $\sim 1 / 3$ of the bait near the front of the trap, and $-1 / 3$ of the bait on the 
ground at the mouth of the srap. Use sardines packed in oil, not mustard, catsup, or some other substance.

9. Avoid trapping on cold nights (i.e., low temperature $\leq 40^{\circ} \mathrm{F}$ ) if there is a high probability $(>20 \%$ ) of precipitation. Wet animals can be injured or killed by hypothermia. Trapping on cold dry nights is acceptable, but many animals are less active in cold weather 80 trap success may be lower.

10. As early ar possible the following morning check each trap. Approach traps cautiously until the presence and identity of the animal is ascertained. If the trap contains a skunk (Mephites spp.), stop immediately and implement the procedure in Sect. 4.3.2.

11. Trzps that do not contain animals may be left in place or semoved. If left in piace, the trap should be sprung to avoid trapping an animal before the trap is rebaited. If the trap is removed, shake all bait and debris out of the trap in the field. If necessary, wash the trap in the stream.

12. Traps that contain target animals should be handled in accordance with Sect. 4.2

13. Nontarget animals, except free ranging cats (Sect. 4.3.1), should be released from the traps immediately. See Sect. 4.3.2 for cietails on releasing skunks. If desired, the trap and animal may be weighed prior to release (this does not apply to skunks) and other notes on animal condition may be made. Wear thick gloves when handling a trap containing an animal.

14. After an animal has been released, remove all debris from the trap. Wear plastic glover when cleaning traps that have held animals.

15. At least once a week, traps are to be rinsed clean, thoroughly disinfected with a bleach solution (see Sect. 4.3.3), and then rinsed again to remove residual bleach. Unremoved bleach will reduce trap success.

\subsection{HIANDLING TARGET ANIMAIS}

The handling of target animals requires the administration of an anesthetic, Ketaset ${ }^{2}$, that must be obtained through the project veterinarian and controlled in accordance with the approved procedure in Attachment A. Ketaset immobilizes the animal, but does not truly anesthetize it. While immobilized and during recovery, the animal is extremely sensitive to light and sound. Therefore, every cffort must be made to keep the animal in a relatively dark area and all unnecessary sounds should be eliminated.

${ }^{2}$ Ketaset is the tradename for a veterinary preparation of ketamine hydrochloride. The preparation is slightly acidic (pH 3.5-5.5) and comes as a liquid $(100 \mathrm{mg} / \mathrm{mL})$ in $10-\mathrm{mil}$ vials. Ketaset is a labeled drug that must be used under the direction of a veterinarian. 
Some details of how target animals are handled will vary according to the individual animal, the purpose of the handling, and the field conditions. The following steps provide guidance on major actions and critical safety requirements. These steps assume that a field worksheet similar to Attachment B is being used.

1. Before approaching the trap, establish an area for handling the animal and prepare the needed materials. This area will usually be the tailgate of the vehicle. Have the ear tattooing equipment ready with the appropriate number inserted. Record the time of arrival at the trap site.

2. Wearing heavy gloves remove the anchoring stake and carry the trap and animal to the preestablished handling area.

3. Weigh the animal and trap and estimate the weight of debris in the trap. Calculate and record the weight of the animal using the field worksheet.

4. Determine the dosage of Ketaset based on the weight of the animal. This dosage is flexible and should be based on the experience of the researchers; however, range of $0.075-0.1 \mathrm{cc} / \mathrm{b}$ should provide rapid anesthesia ( $<5 \mathrm{~min}$ ) and ample time for sampling and tattooing while minimizing the chances of overdosing and the time for recovery. It is better to underdose at first alid administer a second shot, if required, than to overdose.

Ketaset is administered intramuscularly, usually in the thigh. Care must be taken not to inject the drug ints the abdominal or chest cavity of the animal. Two individuals are required for this operation because one person must control sine animal by forcing it into a small area of the trap (using sticks or rebar, not hands) and pin it there until the injection is given. Even with two persons, it is frequently necessary to stick the animal twice to get the complete injection in.

Animals that are groggy may be sampled without complete immobilization if one researcher controls the animal fo head with the catch pole.

5. After the animal is immobilized, remove the animal from the trap (wearing plastic glover or heavy gloves if the animal is not completely immobile). Take care in removing the animal that it is not injured by being scraped over sharp edges and pointed wires.

6. Place the animal on the painter fo drop cloth. Place eye ointment in the open eyes so that the animal f inability to blink does not result in dehydration of the eyes.

7. Trim at least $1 \mathrm{~g}$ of hair from the tail and place in a plastic bag. Label the bag with the trap number, animal's ear tattoo number, and date. At least 1 out of every 10 animals should have enough hair removed to provide for a duplicate sample. The frequency of duplicates may be increased at the discretion of the principal investigator. 
8. Tattoo the animal's ear. The first time an animal is captured, tattoo the right car only. The tattoo should be placed near the edge of the ear to minimize bleeding. A properly tattooed ear should produce no blood. If bleeding occurs, apply rubbing alcohol. Record the ear tattoo number.

9. Check and record the gender of the animal.

10. Record any comments about condition of the animal.

11. Attach radio collar if required.

11. If desired, photograph the animal either while anesthetized or while in trap.

12. Return the animal to the trap until it is completely over the effects of the anesthetic. Animals released while still groggy from the anesthetic may drown, injure themsetves, or fall prey to other animais. If weather conditions and lack of human traffic permit, the trap and animal may be left at the trap site while other traps are investigated, or the trap and animal may be placed in the vehicle and transported to the next trap site(s).

13. Once the animal is fully recovered, release it in the vicinity of the original trap site. Young animals and nursing mothers should be released as close to the trap site as possible. Other animals may be released within a $1 \mathrm{~km}$ of the original trap site, provided that the release point is not near a heavily travelled roadway.

\subsection{CONTINGENCIES}

\subsubsection{Free Ranging Domestic Cats (Felis catus)}

Free ranging cats are highly efficient predators that cause substantial ecological damage through destruction of prey species (especially birds) and competition with natural predators; cats are also a vector for transmission of rabies. The abundance of feral cats on the ORR is unknown, but the population is believed to be small and largely confined to the vicinity of plant buildings. Nevertheless, feral cats have been encountered in remote areas of the ORR, and roadkilled cats have been observed on ORR highways. Trapped cats will be taken in the trap to the Oak Ridge Animal Control Shelter. If the shelter is closed, the TWRA reside'st ageni will be contacted about disposition of the animal.

\subsubsection{Skunles}

Skunks represent an obvious problem when trapped. The following procedure for releasing trapped skunks has been derived from conversations with TWRA and University of Tennessee personnel, but the procedure has not been demonstrated in actual practice on the ORR. 
1. Move vehicles and personnel to a reasonable distance from the trapped animal. All personnel should maintain silence during the release procedure and should avoid any actions that might startle the animal.

2. One person should take the painters' drop cloth, carrying it in front of him as a shield, and cautiously approach the trap.

3. Place the drop cloth gently over the trap so that the animal is in darkness and any spray will be confined. Leave the release end of the trap uncovered.

4. Slowty open the trap and bold the trap open until the skunk has left the trap. Wear gloves during this procedure.

5. If the skunk does not immediately exat the trap, the door may be propped open with a stick (or wired open), and the researcher may move back away from the trap until the skunk exits.

6. Any person or item sprayed by skunk should be rinsed in tomato juice in the field. After field rinsing, the item or individual should return to the nearest facility where water is available to thoroughly wash (shower). Skunk-contaminated clothing should be placed in a large plastic trash sack and disposed of.

\subsubsection{Sick or Injured Animals}

There are a number of sharp edges within the live traps, and trap injuries can be expected. In general, these injuries will involve minor scrapes and cuts. Such minor injuries require no treatment, and the animal should be released after sampling. Occasionally, major trap injuries may occur. Each major injury should be handled on a case-by-case basis. The TWRA agent should be contacted for advice. In some cases, the veterinarian who supplies the anesthetic may be asked for assistance. However, neither ESD nor the ORNL. Biology Division has facilities for surgery and overnight care of wild animals. If neither the TWRA agent nor the veterinarian are available, the Oak Ridge Animal Control Shelter may be contacted. Before any animal is removed from the reservation it must be surveyed by

Radiation Protection. As a last resort, the Principal Investigator may decide to release an injured animal near the trap site.The potential exists for trapping sick animals. The most likely symptoms of sickness are extreme lethargy, diarrhea, or discharges from the nasal or genital areas. Sick animals should be released at the trap site after sampling. Traps that housed a sick animal should be cleaned and disinfected using a solution of bleach that is twice the strength recommended on the bleach bottle. After disinfection, the trap must be thoroughly rinsed with clean water and.allowed to air dry.

\subsection{SAMPLE PREPARATION}

Because the fur of wild animals contains dirt and other contaminants not related to the diet of the animal, it is essential that careful preparation of the hair samples precede submission tor analysis. The following steps represent a minimum protocol for cleanliness. Throughout these steps, the lab worker should wear plastic gloves. Gloves should be washed 
thoroughly, or clanged, between samples to prevent cross contamination. If the hair is expected to be contaminated with radionuclides, then all waste from these steps should be treated as low-level compactible or liquid waste.

1. Prepare work space.

(a) Cleanse lab sink

(b) Place a paper towel or coffee filter in the sink drain to prevent material from entering drain system.

(c) Make sure that deionized water is available from a tap and that the flow can be controlled to a trickle.

(d) Clear a space on the lab bench for drying of hair samples.

(c) Place paper towels or blotter paper on the benchtop and label areas for drying of each sample:

2. Remove hair from plastic bag.

3. Holding hair in one hand, allow a gentle trickle of deionized water to pass through the hair. Repeatedly squeeze the hair.

4. After a minimum of $1 \mathrm{~min}$., check the water flowing through the hair. If the water remains clear and no visiole foreign material remains in the hair, the rinsing process may be terminated. If both conditions are not met, continue rinsing and squeezing until water is clear and hair appears clean.

5. If any hair is washed from the hand into the sink, that lost material may not be used in the sample because of the potential for cross contamination. Reduce the flow of water to eliminate the loss of hair.

6. Squeeze as much water as possible from the hair sample and place the hair on a paper towel or blotter paper (from Step 1.e) to dry. Allow $\geq 16 \mathrm{~h}$ (i.e., overnight) for air drying.

7. After hair is dry, place in a preweighed 15-cc plastic petri dish. Weigh the dish and hair and calculate and record the weight of hair in the sample. Label the dish with the ear tag number, the date of collection, and the weight of hair.

8. Submit the sample to the ESD counting room for gamma counting.

9. Upon receipt of the gamma counting results, record the results, complete an Analytical Services Request form, and submit the sample for mercury and ICP metals analyses. 


\section{PROCEDURE FOR HANDLING AND USE OF KETASET ${ }^{l}$}

As part of a long-term ecological monitoring program, raccoons and mink will be trapped in the White Oak Creek floodplain, the East Fork Poplar Creek floodplain, and in various uncontaminated areas around the Oak Ridge Reservation using wire mesh traps. Traps will be set in the late afternoon and checked early in the moming of the following day. Nontarget animals (e.g., skunk, opossum, fox) will be released immediately. All animals will be released in the immediate vicinity of the trap site within a few hours of capture (i.e., no animals will be kept overnight).

Raccoons and mink will be anesthetized with Ketaset. The animals will be weighed while in the trap to determine proper Ketaset dosage. The recommended dosage for both species is $25-35 \mathrm{mg} / \mathrm{kg}$ body weight ${ }^{2}$, which will be administered by intramuscular injection in the flank area of the animal. Once the animals are immobilized they will be removed from the cages using heavy gloves and a catch-pole. Further examination of the animals will include overall health check, sex determination, ear tagging, removal of a hair sample, and possibly adipose tissue removal depending upon the feasibility of proposed techniques (e.g., liposuction). The animals will be kept under supervision until they are conscious and able to safely leave the capture area.

Some animais will be gamma counted at the Tennessee Wildlife Resources Agency checking station on Bethel Valley Road. Later in the study, some raccoons will be fitted with radio collars.

The primary supply of Ketaset will be kept by the Biology Division Safety Officer (L. L. Triplett). A working supply of up to 2 bottles will be kept in Building 1505 for immediate use by project personnel. Within 1505, the Ketaset will be kept in a locked dratwer in room 144A; this room is used for storage of small quantities of various radionuclides and is kept locked at all times. Access to the drawer will be controlled by the principal investigator (T. L. Ashwood) and will limited to personnel on this project. Access to the radioisotope storage room is controlled by R. K. McConathy, the Environmental Sciences Division Safety Officer. Each time a quantity of the drug is removed from the drawer, a record will be made on the Drug Control Record (DCR). When a bottle is emptied, the empty bottle and associated DCR will be returned to the Biology Division veterinarian (V. L. Godfrey), and a new bottle will be obtained. Copies of all completed DCRs will be retained by the principal investigator.

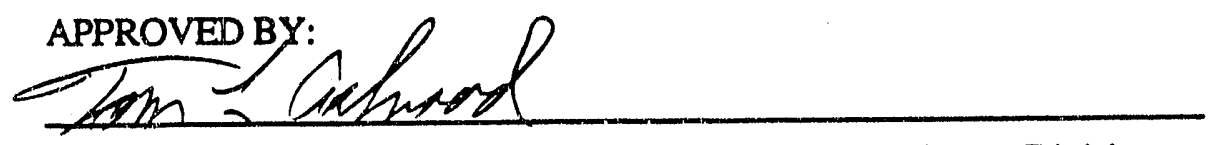

T. L. Ashwood, Principal Investigator, Environmental Sciences Division

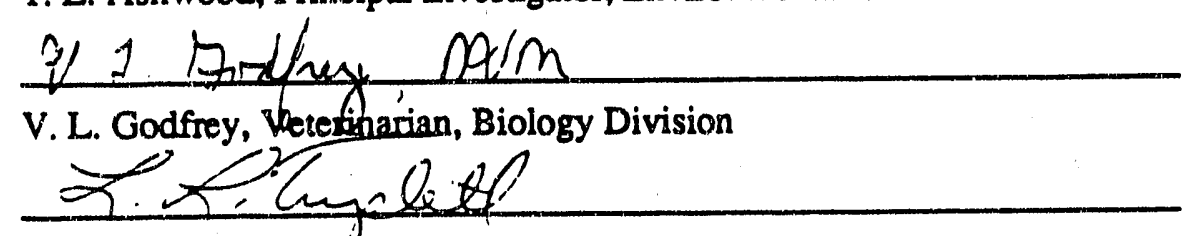

L. L. Triplett, División Safety Officer, Biology Division

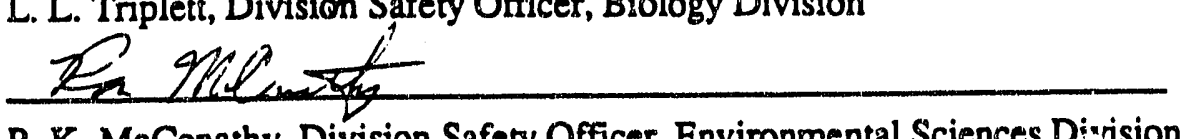

R. K. McConathy, Division Safety Officer, Environmental Sciences Dirision
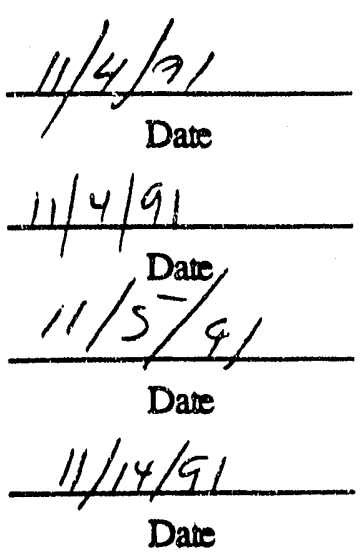

1 Ketaset is the tradename for ketamine hydrochloride, a labelled drug used for anesthetizing animals. The drug comes as a slightly acidic solution (pH $3.5-5.5)$ in $10-\mathrm{mL}$, vials $(100 \mathrm{mg} / \mathrm{mL})$ and must be used under the direction of a licensed veterinarian.

2 This dose is recommended by the Canada Ministry of Natural Resources in their handbook Wild Furbearer Management and Conservation in North America (1987). A dosage of $22 \mathrm{mg} / \mathrm{kg}$ (10 mg/h) has been used successfully on ieccosns ty siudentis and faculty of the Iniversiny of Tennecues Department of Fonestry. Wildlife, and Fisheries. 
C-80

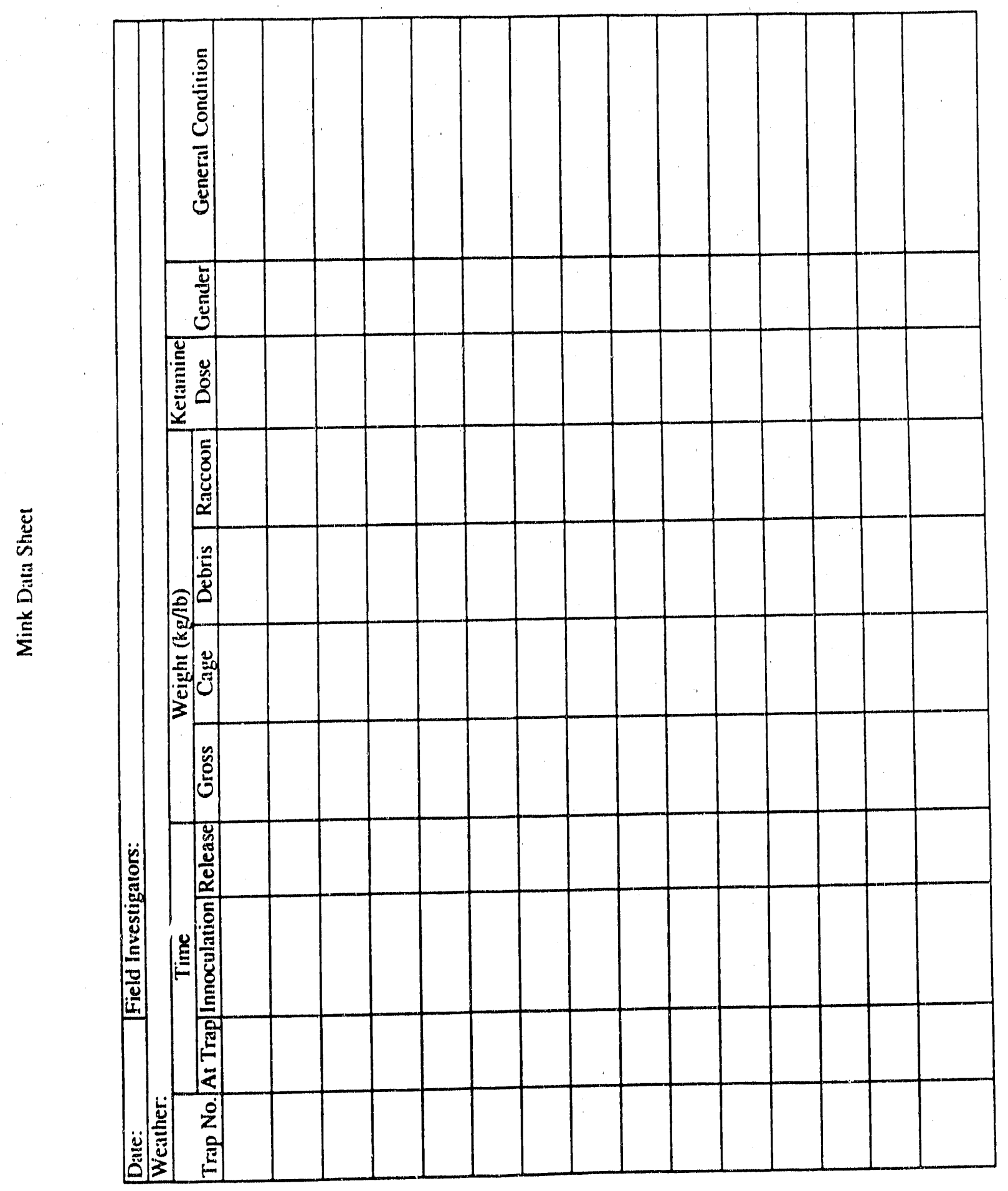




\section{DRUG CONTROL RECORD - RESEARCH}

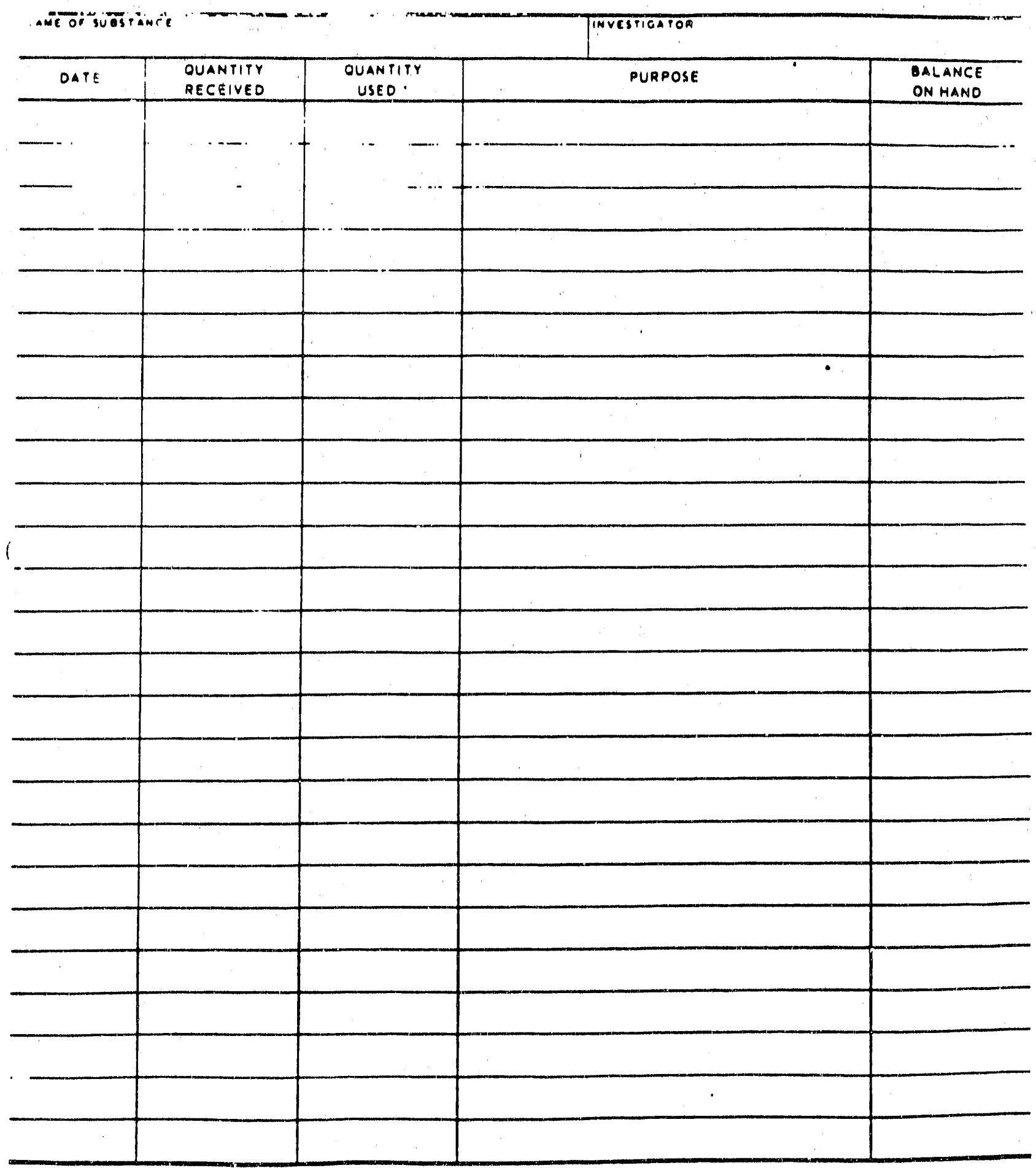




\title{
SURVEYS TO DETERMINE THE EXTENT OF THREATENED AND ENDANGERED SPECIES, WETLANDS, AND FLOODPLAINS
}

\author{
prepared by
}

\author{
T. L. Ashwood, C. J. Hardy, and R. L. Kroodsma \\ Environmental Sciences Division
}

\section{PURPOSE}

To define the actions necessary to determine whether and to what extent threatened and endangered species, wetlands, and bloodplains exist within a specific area.

\section{SCOPE}

This procedure applies to surveys of federally-isted and state-listed plant and animal species that are threatened, endangered, or in need of management. The procedure also addresses surveys to identify the extent of wetlands and floodplains.

\section{PROCEDURES}

Field research personnel are expected to have whatever training is required for access to the study site. Observers who do not have the requisite training may be escorted to the site provided that those responsible for access control have approved the practice of escorting visitors and provided all escort requirements are fulfilled.

Personal protective clothing requirements and personnel monitoring procedures vary from site to site. All field researchers and observers will comply fully with the established procedures for the study site. As a minimum, field researchers and any observers must wear ORNL-provided field clothing (khakis, coveralls, etc.) for study sites on the Oak Ridge Reservation (ORR). Sturdy bnots are required for all field researchers, and wet sites may require rubber boots or waders.

1. The Tennessee Department of Environment and Conservation (TDEC), Tennessee Wildlife Resources Agency (TWRA), and the U.S. Fish and Wildlife Service (FWS) are contacted at least annually to obtain lists of threatened or endangered plant and animal species present in the Oak Ridge area and surrounding region.

2. The ecology and habitat requirements of the listed species, which are generally well known, have been reviewed. Based on this review, a determination has been made for each species as to whether the species occurrence is likely on the Oak Ridge Reservation (ORR) and whether any potentially suitable babitat is present on the ORR. (The presence or absence of habitat types on the ORR is well known.) This determination is updated 
as required by any changes in the lists obtained in step 1 . ORNL reports on the status of listed species on the ORR have been drafted.

3. Records of current and past observations of listed species are maintained.

4. Each proposed construction site or remedial action area is evaluated for the potential presence of listed species based on the information obtained in steps 1-3 above.

5. If a listed species is potentially present on a site, determination is made as to whether and when a field survey is required.

6. Wetlands on the ORR have been generally surveyed, and a report containing this survey information has been issued in draft form.

7. Wetlands surveys are conducted on a site-by-site basis as requested in support of specific projects.

8. All surveys are conducted by competent biologists specifically trained for these surveys.

9. Results of the surveys are documented in letter reports to the requesting authority. 


\title{
FECAL MATERIAL SAMPLING
}

\author{
prepared by \\ T. L. Ashwood \\ Environmental Biotechnology Section
}

\section{PURPOSE}

To define the actions necessary to obtsin fecal material samples (scats) from small carnivores and to prepare those samples for analysis, Although chain of custody procedures will be followed, these samples are intended for reld sereening purposes, only. The objective of the task is to identify animal species that may require further investigation either because they themselves are at risk or because they serve as an ecological indicator of contaminant levels in the Whiteoak Creek floodplain. The data from this study will not be used by itself to evaluate ristes or the need for remedial actions.

\section{SCOPE}

This procedure applies to scat sampling of small carnivores but may be used for a wide range of mammals. The sample preparation steps are primarily applicable to analysis of contaminant levels, and these steps should be reviewed and revised as necessary if other analyses (e.g., food content) are intended.

\section{EQUTPMENT}

1. Surveyor's flagging for identifying sample transects.

2. Rubber gloves.

3. Field clothes appropriate to the field site.

4. Small $(15 \mathrm{~cm})$ plastic ruler.

5. Large forceps.

6. Preweighed 15-ce plastic petri dishes for samples (tare weight must be marked on dish).

7. Yellow plastic tape.

8. Permanent ink marker.

9. Field notebook

10. Scat and track identification guides.

11. Plastic trash bag.

12. Sturdy bag (e.g., daypack) for carrying equipment and samples.

13. Camera (optional). 


\section{PRC'JEDURES}

A major bealth concern related to handling of wild animal feces is transfer of parasites, particularly the round worm Baylis asceris', from animals or their scats to humans. All personnel handling scats must wear plastic gloves during this handling. As s0on as possible after handling any scats, each individual must thoroughly wash their hands and arms to the elbow with soap and hot water. All clothing that has been in contact with the scats must also be laundered, and care should be taken to keep hands, clothing, or any potentially contaminated items away from the face. Eating, drinking, chewing tobacco, smoking, or applying cosmetics are to he avoided while in the field, unless the individual is able to thoroughly wash in the field.

Field research personnel are expected to have whatever training is required for access to the study site. Otnervers who do not have the requisite training may be escorted to the site provided that those responsible for access control have approved the practice of escorting visitors and provided all escort requirements are fulfilled.

Personal protective clothing requirementu and personnel monitoring procedures vary from site to site. All field researchers and observers will comply fully with the eatablished procedures for the study site. As a minimum, field researchers and any observers must wear ORNL-provided field clothing (khakis, coveralls, etc.) for study sites on the Oak Ridge Reservation (ORR). Sturdy boots are required for all tield researchers, and wet sites may require rubber boots or waders.

\subsection{SAMPLE COLLECTION}

1. Prior to sample collection, the field site should be visually surveyed in detail to identify signs of the target animal(s). Based on such signs and on a knowledge of the babits of the animal(s), one or more transects should be laid out and marked with the flagging. This step should be accomplished a few days prior to sample collection to allow the signs of human interference (primarily rcent) to disappear.

2. In an orderly fashion, follow the transect(s) carefully looking for scats.

3. Scats that are found should be identified as chosely as possible to a species using the field guides available for this purpose. Scats that carnot be identified closer than family (c.g., Mustelidae) should not be collected. If desired, photos of the acat and surrounding site may be taken.

1 Baylis asceris may be contracted from the scat or fecal material of raccoons and is always fatal when contracted by humans. 
If photo are taken, the roll of film must be marked with a unique identification number, and the frame number of each photo must be recorded, along with the roll number, in the field notebook.

4. It is critical that all factors considered in scat identification be recorded in the field notebook. Also record the location of the scat, the color, degree of dryness, presence of other sign (e.g., tracks, fur), and any other information that may be of use in confirming the identification or in evaluating the results (for instance, old dried scats may have lost contamination through leaching by rain).

5. Uring the forceps, place the scat sample into a petri dish that has already been marked with the date, probable species, location, and unique sample identifier. It is essential that the sample completely fill the petri dish. If there is insufficient material to fill the dish, then the proportion of the dish (e.g., one half) filled must be noted on the container. Seal the dishes with yellow plastic tape.

6. If there is sufficient material, prepare duplicate samples.

7. If a field survey instrument is available, survey the scat samples in the field and record the reading (counts per minute above background) on the bag and in the field notebook If a field survey instrument is not available, survey the samples upon returning to the laboratory and record the same information.

8. Upon returning to the lab, weigh the sample dishes and calculate the fresh weight of the sample. Mark this weight on the dish and in the field notebook. Place the samples in a locked freezer and complete chain of custody forms.

\subsection{SAMPLE PREPARATION}

Because the scats of wild animals may contain dirt and other contaminants not related to the diet of the animal, it is essential that careful preparation of the samples precede submission for analysis. The following steps represent a minimum protocol for cleanliness. Throughout these steps, the lab worker should wear plastic gloves. Gloves should be washed thoroughly, or changed, between samples to prevent cross contamination. If the scat is expected to be contaminated with radionuclides, then all waste from these steps should be treated as low-level compactible or liquid waste.

1. Contact the lab steward for the ESD counting room and arrange a schedule for gamma counting of the samples. All scat samples are to be counted for $\leq 1000 \mathrm{~min}$ (i.e., overnight). Provide petri dishes (still frozen) in a plastic bag on the agreed upon schedule.

2. After a sainple has been gamma counted, return the sample to the locked freezer. 
3. When a sufficient number of samples have been counted to provide a reasonable batch size for chemical analysis (i.e., $\geq 10$ ), remove the samples from the freezer and split each sample into two equal portioni. Place each portion into a plastic scintillation vial labelled with the sample number, collection date, sample matrix (i.e., feces), and preservation (i.e., frozen).

4. Complete sample forms and submit samples to Analytical Chemistry Division for anatysis. One vial should be analyzed for mercury, and the second vial should be anatyzed for PCBe and organochlorine pesticides (by capillary column gas chromatography). 


\section{DISTRIBUTION}

1-2. T. L. Ashwood

3. L. V. Asplund/N. W. Durfee

4. L. D. Bates

5. D. M. Borders

6-10. H. L. Boston

11-12. V. Chidambariah

13. K. W. Cook

14. J. H. Cushman

15-16. D. J. Downing

17-18. T. A. Fontaine

19. D. E. Fowler

20. S. B. Garland II

21. C. W. Gehrs

22. C. D. Goins

23. S. G. Hildebrand

24. L. D. Hyde

25. P. Kanciruk

26-27. R. H. Ketelle

28. A. J. Kuhaida, Jr.

29-30. S. Y. Lee

31. V. Legg

32-33. D. E. Miller

34-35. G. K. Moore
36-37. P. T. Owen

38. D. E. Reichle

39. G. E. Rymer

40. P. A. Schrandt

41. F. E. Sharples

42. D. S. Shriner

43. S. H. Stow

44-45. G. W. Suter

46. D. W. Swindle

47-48. M. F. Tardiff

49. R. I. Van Hook

50. D. R. Watkins

51-52. J. A. Watts

53. K. K. White

54-55. D. S. Wickliff

56-60. ER Document Management Center

61. Central Research Library

62-66. ESD Library

67. ORNL Y-12 Technical Library

68. Laboratory Records Dept.

69. Laboratory Records, ORNL-RC

70. ORNL Patent Section

71. Office of Assistant Manager for Energy Research and Development, DOE Field Office, Oak Ridge, P.O. Box 2001, Oak Ridge, TN 37831-8600

72. P. H. Edmonds, Radian Corporation, 120 South Jefferson Circle, Oak Ridge, TN 37830

73. J. F. Franklin, Bloedel Professor of Ecosystem Analysis, College of Forest Resources, University of Washington, Anderson Hall (AR-10), Seattle, WA 98195

74. G. M. Hornberger, Professor, Department of Environmental Sciences, University of Virginia, Charlottesville, VA 22903

75. G. Y. Jordy, Director, Office of Program Analysis, Office of Energy Research, ER-30, G-226, U.S. Department of Energy, Washington, DC 20545

76-79. W. E. Murphie, Department of Energy, Office of Environmental Restoration, Eastern Area D\&D Branch, EM-423 (GTN), Washington, DC 20545

80. R. H. Olsen, Vice President for Research, University of Michigan, Medical Science Building II, No. 5605, 1301 East Catherine Street, Ann Arbor, MI 48109-0620

81. A. Patrinos, Acting Director, Environmental Sciences Division, Office of Health and Environmental Research, ER-74, U.S. Department of Energy, Washington, DC 20585

82-83. S. S. Perkins, DOE Field Office, Oak Ridge, Information Resource Center, 105 Broadway, Oak Ridge, TN 37830

84-85. S. P. Riddle, DOE Field Office, Oak Ridge, P.O. Box 2001, Oak Ridge, TN 37831-8541

86-87. R. C. Sleeman, DOE Field Office, Oak Ridge, P.O. Box 2001, Oak Ridge, TN 37831-8541

88. F. J. Wobber, Environmental Sciences Division, Office of Her lth and Environmental Research, Office of Energy Research, ER-74, U.S. Department of Energy, Washington, DC 20584

89-90. Office of Scientific and Technical Information, P.O. Box 62, Oak Ridge, TN 37831 

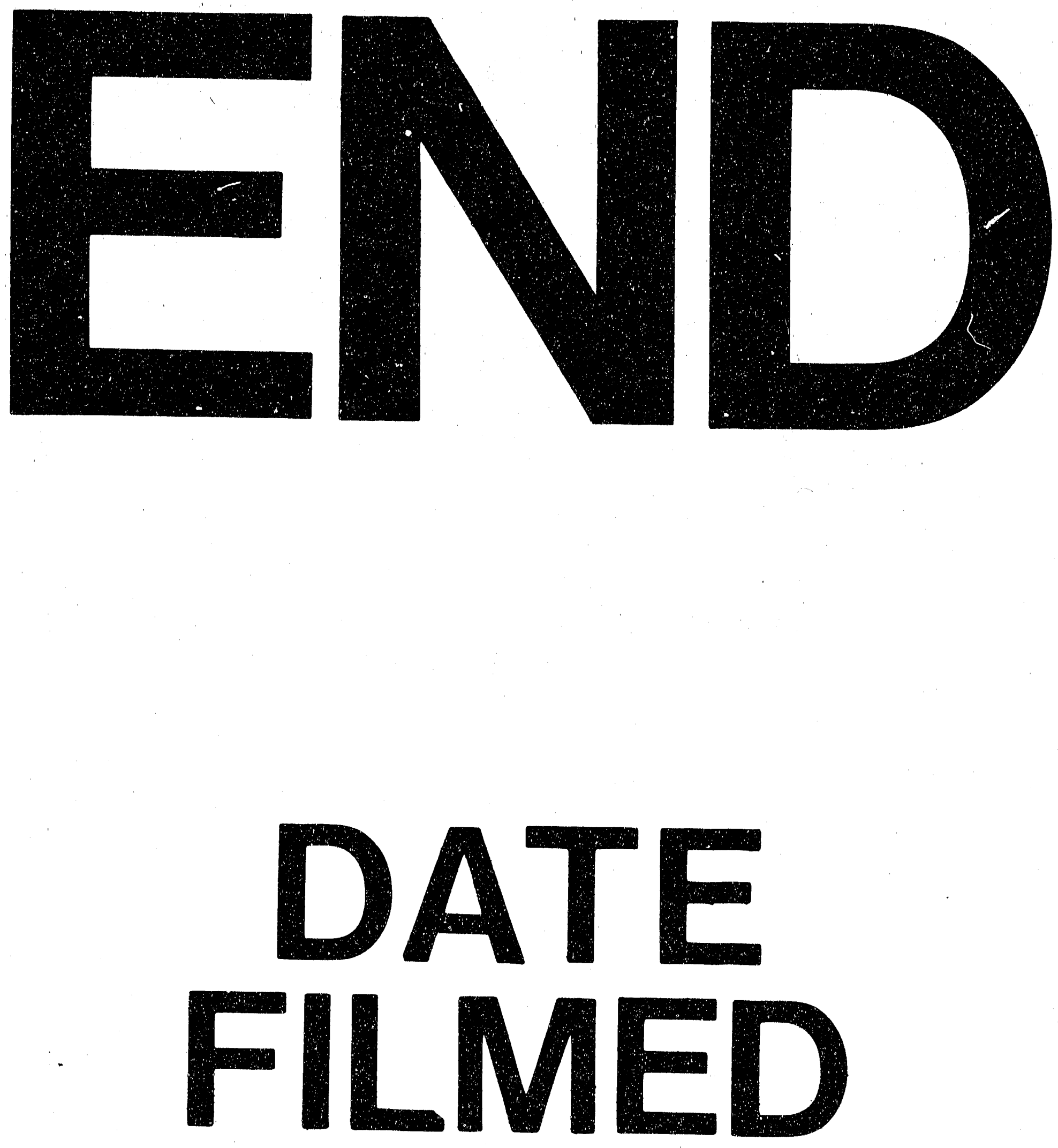

咅

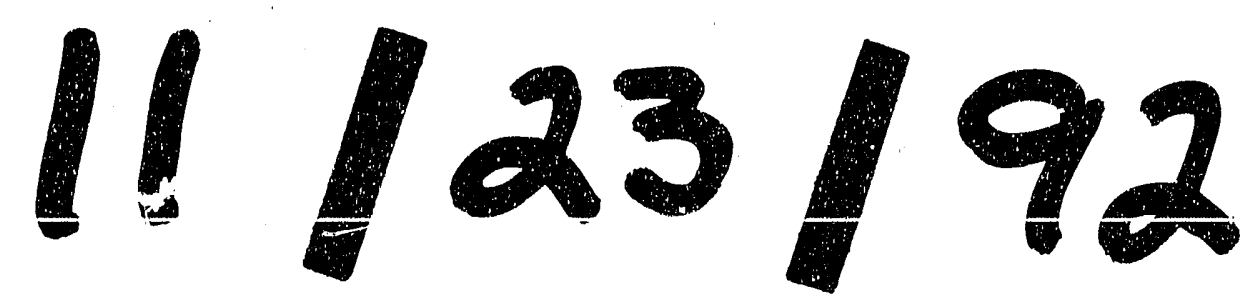


